\title{
Improving HPV vaccination acceptability by a web- based tailored intervention
}

Citation for published version (APA):

Pot, M. (2018). Improving HPV vaccination acceptability by a web-based tailored intervention. [Doctoral Thesis, Maastricht University]. Maastricht University. https://doi.org/10.26481/dis.20181005mp

Document status and date:

Published: 01/01/2018

DOI:

10.26481/dis.20181005mp

Document Version:

Publisher's PDF, also known as Version of record

\section{Please check the document version of this publication:}

- A submitted manuscript is the version of the article upon submission and before peer-review. There can be important differences between the submitted version and the official published version of record.

People interested in the research are advised to contact the author for the final version of the publication, or visit the DOI to the publisher's website.

- The final author version and the galley proof are versions of the publication after peer review.

- The final published version features the final layout of the paper including the volume, issue and page numbers.

Link to publication

\footnotetext{
General rights rights.

- You may freely distribute the URL identifying the publication in the public portal. please follow below link for the End User Agreement:

www.umlib.nl/taverne-license

Take down policy

If you believe that this document breaches copyright please contact us at:

repository@maastrichtuniversity.nl

providing details and we will investigate your claim.
}

Copyright and moral rights for the publications made accessible in the public portal are retained by the authors and/or other copyright owners and it is a condition of accessing publications that users recognise and abide by the legal requirements associated with these

- Users may download and print one copy of any publication from the public portal for the purpose of private study or research.

- You may not further distribute the material or use it for any profit-making activity or commercial gain

If the publication is distributed under the terms of Article $25 \mathrm{fa}$ of the Dutch Copyright Act, indicated by the "Taverne" license above, 


\section{Improving \\ HPV VACCINATION ACCEPTABILITY \\ by a Web-based tailored intervention}

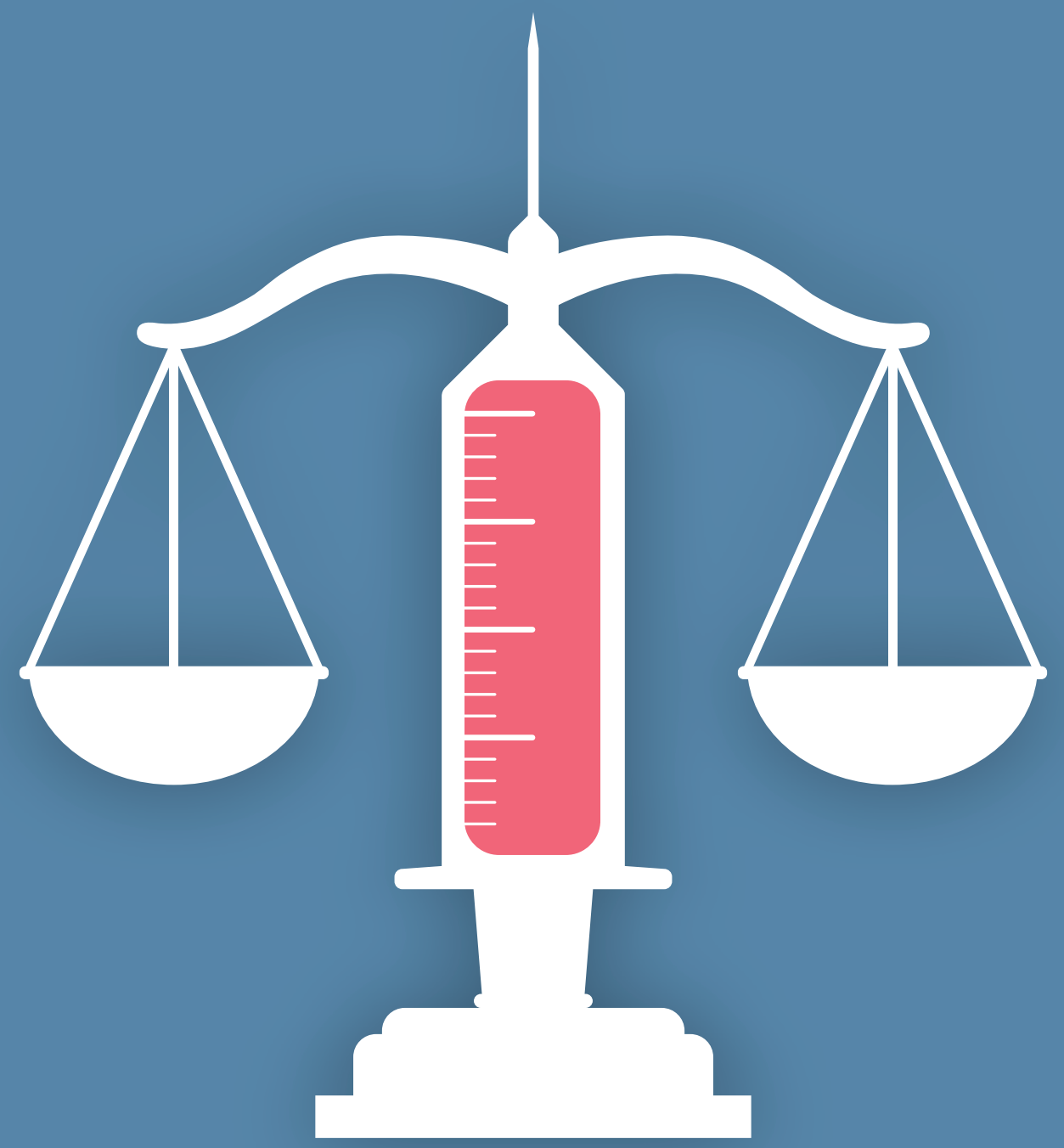

MIRJAMPOT 

IMPROVING HPV VACCINATION ACCEPTABILITY

BY A WEB-BASED TAILORED INTERVENTION 


\section{COLOPHON}

The research presented in this $\mathrm{PhD}$ thesis was conducted at the Netherlands Organization for Applied Scientific Research TNO (Department of Child Health), and carried out in collaboration with Maastricht University (Department of Work and Social Psychology), and the National Institute for Public Health and Environment (RIVM).

The studies were financially supported by a grant from the Netherlands Organization for Health Research and Development (ZonMw) (grant-number 50-51515-98-25).

ISBN:

978-94-6375-008-0

Lay-out \& cover design: $\quad$ Design Your Thesis, www.designyourthesis.com

Print:

Ridderprint, Ridderkerk, the Netherlands

\section{Copyright (C) 2018 Mirjam Pot, Amsterdam}

All rights reserved. No part of this publication may be reproduced or transmitted in any form or by any means, electronical or mechanical, including photocopying, recording, or any information storage and retrieval system, without written permission from the author. 


\title{
IMPROVING HPV VACCINATION ACCEPTABILITY BY A WEB-BASED TAILORED INTERVENTION
}

\author{
P R O E F S C H R I F T
}

ter verkrijging van de graad van doctor aan de Universiteit Maastricht, op gezag van de Rector Magnificus, Prof.dr. Rianne M. Letschert volgens het besluit van het College van Decanen, in het openbaar te verdedigen op vrijdag 5 oktober 2018 om 12.00 uur

door

Mirjam Pot 


\section{PROMOTOR}

Prof. dr. R.A.C. Ruiter

\section{COPROMOTORES}

Dr. H.M. van Keulen (TNO)

Dr. T.G.W.M. Paulussen (TNO)

\section{BEOORDELINGSCOMMISSIE}

Prof. dr. G. Kok (voorzitter)

Dr. R. Crutzen

Prof. dr. C.J.P.A. Hoebe

Prof. dr. L. Lechner (Open Universiteit)

Prof. dr. M. Prins (Universiteit van Amsterdam) 


\section{TABLE OF CONTENTS}

CHAPTER 1 General Introduction

CHAPTER 2 Motivational and contextual determinants of HPV vaccination uptake: A longitudinal study among mothers of girls invited for the HPV vaccination

CHAPTER 3 Experimental pretesting of statistical and narrative risk information on mothers' perceived daughters' susceptibility towards HPV-related risks

CHAPTER 4 Systematically Developing a Web-based Tailored Intervention Promoting HPV vaccination Acceptability among Mothers of Invited Girls using Intervention Mapping

CHAPTER 5 Effectiveness of a Web-based tailored intervention with virtual assistants promoting the acceptability of HPV vaccination among mothers of invited girls: A randomized controlled trial

CHAPTER 6 General Discussion

$\begin{array}{lll}\text { CHAPTER } 7 & \text { References } & 133\end{array}$

Appendices 151

Valorization Addendum $\quad 173$

Summary 175

Samenvatting $\quad 179$

Dankwoord 183

Curriculum Vitae 185 



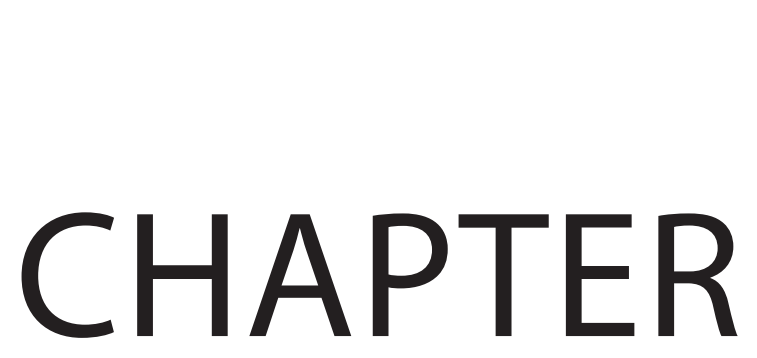

General Introduction 



\section{GENERAL INTRODUCTION}

\section{Cervical cancer and HPV}

Cervical cancer is the fourth most common cancer among women worldwide. Globally, in 2012, there were 527,624 new cases and 265,672 deaths caused by cervical cancer (GLOBOCAN, 2012). Persistent infection by the human papillomavirus (HPV) appears the major cause of cervical cancer; nearly all cases (over 99\%) are attributable to an HPV infection (Bosch et al., 2002; Schiffman \& Castle, 2003). Besides, there is an increasing body of evidence that strongly links HPV infection with cancers of the anus, vulva, vagina, penis, and head and neck (e.g., Chaturvedi et al., 2011; Gillison et al., 2008). HPV is a sexual transmitted infection (STI) and is mainly transmitted through direct sexual contact such as anal, vaginal, or oral sex (Kjaer et al., 2001; Kjaer et al., 2005). However, since HPV is a skinto-skin infection, intercourse is not required to contract the infection. Therefore, condoms may not fully protect against HPV infection (Manhart \& Kouts ky, 2002). It is estimated that $80 \%$ of all sexually active individuals will have an HPV infection during her or his life (Einstein et al., 2009). Women have a 30-50\% chance of acquiring an HPV infection during their first years of sexual activity (Winer et al., 2008).

There are two types of HPV: high-risk and low-risk HPV. The high-risk HPV 16 and 18 are responsible for approximately 70\% of all cervical cancers (Munoz et al., 2004). Low-risk HPV sause approximately $90 \%$ of genital warts (Doorbar et al., 2012). The majority of all HPV infections (approximately 90\%) are cleared rapidly by the immune system and do not progress into cervical cancer (Giuliano et al., 2011; Moscicki et al., 2012; Winer et al., 2011). But, the high-risk HPV types usually require more time before they are cleared compared to the low-risk types (Giuliano et al., 2015). Persistent infection with high-risk HPV types may progress to cell abnormalities in the cervix (Giuliano et al., 2011; Moscicki et al., 2012; Winer et al., 2011). If untreated, these precancerous lesions may progress to invasive cancer. However, even when severely abnormal cells are seen on the cervix (a condition called cervical intraepithelial neoplasia 3, or CIN3), these do not always lead to cancer. The percentage of CIN3 lesions that progress to invasive cervical cancer has been estimated to be $50 \%$ or less (McCredie et al., 2008). It typically takes 10-20 years for an initial HPV infection to develop into full blown cancer (Weinberg, 1999).

\section{Cervical Cancer Prevention}

Secondary prevention by means of national cervical screening programs has greatly reduced the burden of cervical cancer (Franco et al., 2001). These programs aim to prevent cervical cancer by detecting and treating cervical abnormalities before they cause problems. In the Netherlands, since the 1980s, a cervical cancer screening program has existed for 
women aged 30-60 years (Braspenning et al., 2001). Once every five year, these women are invited to undergo screening. When pre-cancer is diagnosed, this is treated. However, despite the screening program, 750 new cases of cervical cancer are still detected yearly in the Netherlands, of which 242 with fatal consequences (Bruni et al., 2016). Besides, a screening program has little impact on circulation and transmission of the HPV virus in the population. After all, preventive measures against an infection with HPV such as safe sex seem to be insufficient (Manhart \& Koutsky, 2002). Therefore, there is a need for additional preventive measures in order to reduce the cervical cancer burden.

Since 2006, a vaccine protecting against HPV 6,11, 16 and 18 is available $\left(\right.$ Gardasil $^{\varpi}$ ), and since 2007 a vaccine protecting against HPV 16 and 18 is available (Cervarix ${ }^{\otimes}$ ). The vaccines stimulate the body's immune system to produce antibodies, which bind to the virus in future encounters with HPV. This prevents the virus from infecting cells (European Medicines Agency, 2014). Both vaccines have showed high efficacy against HPV 16 and 18 (Castellsagué et al., 2011; Lehtinen et al., 2012). Hence, the HPV vaccination enables primary prevention of cervical cancer (Arrossi et al, 2017).

\section{The Dutch National Immunization Program}

In the Netherlands, childhood vaccinations against infectious diseases are offered free of charge by the National Immunization Program (NIP). Vaccinations are voluntary and are offered at different time points during a child's lifetime. Between the ages of 0 and 4 years, children receive the appropriate vaccinations at the Child Welfare Center of the Public Health Service during periodic consultation. During this consultation, they also receive free-of-charge health check-ups (Van Lier et al., 2012). When they reach the age of 9 , children are offered to receive the vaccinations against diphtheria, tetanus, and polio (DTP) and measles, mumps, and rubella (MMR; in Dutch BMR) (Van Lier et al., 2012). The DTP and MMR vaccines are administered at public venues designated by the Public Health Service, such as sports centers and child-and-parent centers. During this vaccination moment, parents are not offered a personal consult. Overall, participation in the NIP is high with a coverage rate of around 95\% (Van Lier et al., 2016).

The Minister of Health decides on (not) including new vaccines into the NIP and which target group should undergo vaccination. These decisions are based on recommendations of the Dutch Health Council (an independent scientific advisory board for the Ministry of Health, Welfare and Sports). The NIP is being managed by the National Institute of Public Health and the Environment (RIVM). RIVM is also responsible for providing information about the NIP to child vaccine providers and parents. 


\section{HPV vaccination in the NIP}

Following advice from the Dutch Health Council in March 2008, the Minister of Health decided to introduce routine HPV vaccination (Cervarix ${ }^{\circledR}$ ) in the NIP. The HPV vaccination is voluntary and is offered free of charge, similar to the other vaccinations offered by the NIP. But, this vaccination targets a new age group (i.e., 12 years) and is only available for girls. This target group was chosen as the HPV vaccination is preferably given prior to the initiation of sexual activity, because the degree of protection is reduced after HPV infection (Koutsky et al., 1992; Woodman et al., 2001).

Initial implementation started with a catch-up campaign in 2009 for girls aged 13-16 years. From 2010, new cohorts of 12-year-old girls have been invited by the NIP to receive the HPV vaccination on a yearly base. Similar to the DTP and MMR vaccine, local group sessions are organized by the municipal health services for receiving the HPV vaccination. These sessions usually take place at large venues (e.g., sport centers). The vaccination itself is given by youth health professionals. Because of the large numbers, there is little privacy and time for interaction between the parent/girl and these professionals. The schedule includes two injections with a six month interval. In the Netherlands, no parental permission is needed for 12-year-old girls to make a decision about uptake.

Before implementation, HPV vaccination uptake was expected to be lower (70\%) compared to the uptake rate usually found for other childhood vaccinations in the Netherlands (around 95\%). This lower uptake was expected because the HPV vaccination was new, targeting a sexual transmitted disease, and it was offered to a new age group and only to girls (Rondy et al., 2010). Although there has been a slight increase in uptake ranging from $52 \%$ in 2009 to $61 \%$ in 2015, the uptake has declined with $8 \%$ in 2016 (Van Lier et al., 2017), and there has been a further decrease in 2017 (i.e., the uptake was 46\%) (Van Lier et al., 2018).

Improving uptake remains critical in order to reduce the cervical cancer burden. Hence, the Dutch Health Council has stated that a need exists for interventions aimed at increasing HPV vaccination uptake.

\section{Interventions promoting HPV vaccination}

In the Netherlands, girls and their parents are provided with an introduction folder and a link to a website providing universal information about HPV and HPV vaccination. These are sent together with the regular invitation for the HPV vaccination. However, the existing intervention seems to be insufficient in promoting HPV vaccination, as is reflected in the current moderate uptake. Hence, the existing interventions promoting HPV vaccination need to be improved. 
Internationally, several interventions to promote HPV vaccination have been tested. Recent reviews on the effects of such interventions showed that one of the most effective singlemethod intervention strategy was a "reminder and recall system". This includes phone calls, text messages by phone, (e-)mailed letters, and/or outreach visits (Fu et al., 2014; Niccolai \& Hansen, 2015; Smulian, Mitchell, \& Stockley, 2016; Walling et al., 2016). However, while such single strategies can be effective, interventions may be most successful when implemented in combination with each other to address multiple barriers to vaccination (Smulian, Mitchell, \& Stockley, 2016). Furthermore, they reported that school-based interventions can reach high uptakes as long as the community provides support and the vaccine is available within schools (Walling et al., 2016). Finally, the effects of educational interventions remain to be mixed (Fu et al., 2014; Niccolai \& Hansen, 2015). The authors of a recent systematic review concluded that no clearly superior educational interventions were identified meriting recommendation for widespread implementation (Fu et al., 2014). Studies that were both well-designed and had adequate power to detect a change in HPV vaccination uptake were rare and generally did not prove the tested intervention to be effective (Fu et al., 2014).

As yet, there is a need for more research on the development of theory- and evidence based interventions promoting HPV vaccination that can be applied in all type of settings (Smulian, Mitchell, \& Stockley, 2016). Previous interventions aimed at improving HPV vaccination uptake have, to our knowledge, never been developed in such a systematic manner. Therefore, the objective of this dissertation is to systematically develop and evaluate an intervention promoting HPV vaccination acceptance. For this, we used the Intervention Mapping (IM) protocol.

\section{Systematic intervention development: Intervention Mapping}

IM provides a framework for the planning of theory- and evidence-based health promotion interventions. It describes the path from problem identification to solution (Bartholomew Eldredge et al., 2016). There are six iterative steps in the IM process: (1) the conduction of a needs assessment and formulation of the overall goals of the intervention; (2) the definition of proximal intervention outcomes and change objectives; (3) the design of the intervention program including the selection of theory-based intervention methods and practical applications; (4) the actual production of the intervention program; (5) the anticipation of intervention adoption, implementation, and sustainability; and (6) the planning of a process and effect evaluation. The IM protocol can be used to ensure a solid foundation of the intervention to be developed and to maximize the likelihood of effectiveness (Bartholomew Eldredge et al., 2016). In line with the IM protocol, the present 
PhD thesis can be divided into three parts: (1) the needs assessment carried out to explain HPV vaccination acceptability (Chapter 2), (2) the development of the intervention (Chapters 3 and 4), and (3) the evaluation of the intervention (Chapter 5).

\section{PART I: THE NEEDS ASSESSMENT}

\section{Target group}

Most studies have confirmed that parents play a large role in the decision-making process about their daughters' HPV vaccination (e.g., Brewer \& Fazekas, 2007; Dahlstrom et al., 2010; Gerend et al., 2009). As mentioned before, in the Netherlands, girls are legally allowed to make their own decision about their HPV vaccination. However, research has shown mothers to play a most important role in the immunization decision of these girls (Van Keulen et al., 2010). When mothers and daughters were subdivided into three groups (i.e., positive intention / neutral intention / negative intention), there was a high percentage of consensus between mothers and daughters (79\%) about the outcome (Van Keulen et al. 2010). Only a very small percentage of the daughters (4\%) had an intention opposing their mothers'. Therefore, mothers are the most important target group for interventions promoting HPV vaccination acceptability.

\section{Determinants of (HPV) vaccination}

Different theories of health behavior have been used to explain vaccination behavior, with the 'Theory of Planned Behavior' (TPB) (Ajzen, 1985) (most recent version:' Reasoned Action Approach) (Fishbein \& Ajzen, 2010), 'Health Belief Model' (HBM) (Becker, 1974), and Social Cognitive Theory (SCT) (Bandura, 1976), being the most prominent frameworks. The TPB proposes that the most proximal determinant of behavior is intention, which, in turn, is guided by three constructs: attitudes toward the behavior (i.e., the evaluation of advantages and disadvantages of a behavior), social norms (i.e., the perceived approval or support of others) and perceived behavioral control (i.e., the perceived ease of performing a behavior) (Ajzen, 1985). According to the HBM, behavior is influenced by perceived susceptibility for and perceived severity of a negative health condition, perceived benefits and barriers of possible actions to avoid the negative condition, cues to action (e.g., reminders to take action) and self-efficacy expectations (Becker, 1974). The Social Cognitive Theory suggests that behavior is a result of the interaction between environmental (e.g., social support, rewards) and personal factors (e.g., knowledge, skills; Bandura, 1986).

As intervention targets are best found trough 'modifiable' determinants, we focused on social psychological determinants (Bartholomew Eldredge et al, 2016). Reviews have demonstrated the importance of social-psychological determinants in explaining HPV 
vaccination behavior (e.g., See Brewer \& Fazekas, 2007). Several Dutch studies into social psychological determinants of HPV vaccination acceptance have been conducted. Van Keulen and colleagues (2013a; 2013b; 2010) found HPV vaccination acceptability to be associated with attitudes, beliefs, outcome expectancies, anticipated regret, confidence in authorities, habit strength, risk perception about being or not being vaccinated, subjective and descriptive norms, relative effectiveness of the HPV vaccination, ambivalence, and processing of HPV vaccination education. The most dominant attitude-based beliefs were beliefs about the safety and effectiveness of the HPV vaccine, (mis)perceptions about sexual maturation and future sexual risk taking of their daughter, perceived economic profit for the pharmaceutical industry, and trust in the government's policies with respect to prevention of infectious diseases (Van Keulen et al., 2013a; Van Keulen et al., 2013b; Van Keulen et al., 2010). The association between such attitude-based beliefs (e.g., concerns about the side effects) and HPV vaccination acceptance / rejection was confirmed by Genefaite and collegues (2012). Finally, Hofman and colleagues (2014) conducted a study in which they longitudinally verified the role of anticipated regret in predicting HPV vaccination uptake. In addition, HPV vaccination intention and a later (2011) versus earlier (2010) decision were also found to be strong predictors of uptake.

The theories and empirical findings described above provided us the theoretical framework for the needs assessment. The framework is presented in Figure 1.1. We made a distinction between proximal (e.g., attitude and intention; see Figure 1.1) and distal (e.g., trust in authorities and ambivalence, see Figure 1.1) determinants; the latter were expected to influence behavior via the proximal determinants. Except for the study conducted by Hofman and colleagues (2014), the studies conducted so far, were cross-sectional in nature. Therefore, in order to verify the extent to which the determinants found in the earlier crosssectional studies predict future HPV vaccination uptake, we conducted a longitudinal study (Chapter 2). The results from this study provided us with the departure points for developing the intervention: determinants that appeared both relevant and changeable were selected as intervention targets.

\section{Informed decision-making and decisional conflict}

Next to HPV vaccination uptake and its determinants, informed decision-making and decisional conflict also turned out to be relevant intervention targets. Research has shown that large proportions of the mothers do not actively acquire and process information about the pros and cons of the HPV vaccination and feel ambivalent about their decision (Van Keulen et al., 2013a; Van Keulen et al., 2010). This indicates these decisions are based on rather unstable grounds, which makes them vulnerable for arguments challenging their initial attitudes and/or intention. Informed decision-making is expected to make mothers 
less vulnerable for such counter arguments. Furthermore, decisional conflict appears strongly related to informed decision-making as decisional conflict may arise when feeling uninformed (O’Connor, Jacobsen, \& Stacey, 2002).

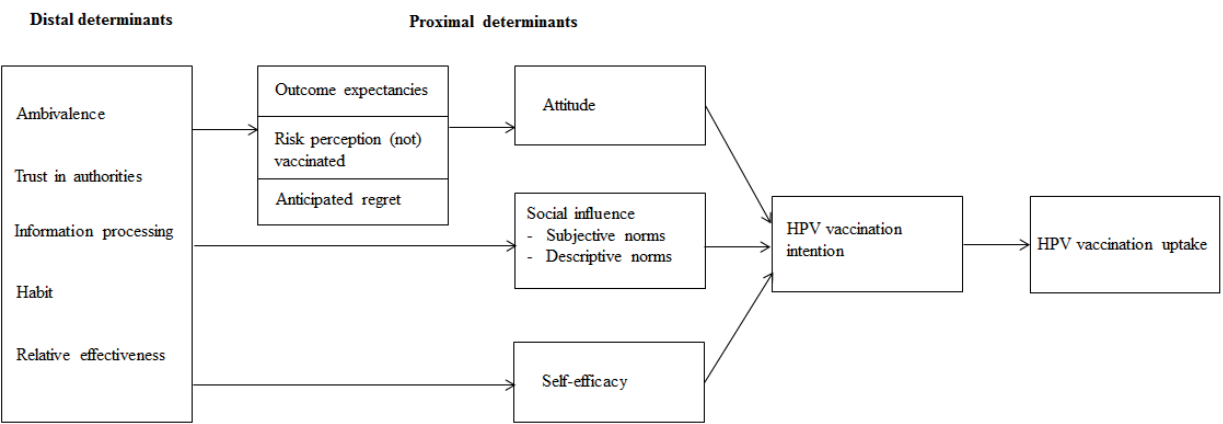

Figure 1.1 Theoretical Framework.

\section{Preferences for HPV vaccination communication}

Next to insight into determinants of HPV vaccination acceptance (Chapter 2), research has shown that mothers have certain preferences concerning the communication about the HVP vaccination. Regarding the delivery mode, mothers indicated a preference for the Internet (Van Keulen et al., 2010). Also, a need existed for more interactive, personal information compared to the universal one-sided communication approach used so far in the Netherlands. Furthermore, mothers expressed differential needs concerning the amount and scope of information (Van Keulen et al., 2010). These preferences guided our decision to develop a Web-based intervention, enabling us to provide mothers with interactive, tailored information about the HPV vaccination.

\section{PART II: INTERVENTION DEVELOPMENT}

Chapter 3 and 4 describe the systematical development of the interactive, Web-based intervention promoting HPV vaccination.

\section{eHealth interventions}

With continued advances in Web technology, the number of computer and Web-based -or so called 'eHealth interventions' - targeting health behavior change has grown rapidly over the past years (Noar \& Harrington, 2012). Computer technology has numerous advantages, 
including interactivity and multimedia features, the ability to deliver individualized content, and automated delivery of interventions. The Internet brings with it the possibility to reach large proportions of people at relatively low costs when compared to interpersonal communication (Noar \& Harrington, 2012). Several meta-analyses have demonstrated these added values of eHealth interventions (Crutzen, Kohl, \& de Vries, 2013; Griffiths et al., 2006). Examples can be found within the context of cancer screening (Jerant et al., 2007; Russell et al., 2010), decision-making in cancer treatment (Jibija-Weiss et al., 2006; Lee et al., 2010), use of healthcare services by newly diagnosed persons with cancer (Loiselle \& Dubois, 2009), and HIV/STI prevention (Noar \& Willoughby, 2012).

\section{User-centered design}

To maximize compatibility with the needs of the target group, a user-centered design strategy by means of focus groups and online experiments was applied. This entails the iterative involvement of the end-users (i.e., mothers) in the design process (Mao et al., 2005). We gathered feedback on different versions (static and interactive) or (parts of the) intervention, by online experimental pretests and focus groups respectively. According to IM, pretesting of intervention components is an important element of intervention development (Bartholomew Eldredge et al., 2016). Experimental pretesting, in specific, enables researchers to draw reliable conclusions about the effectiveness of developed materials in influencing predetermined objectives. In addition, it serves as a guarantee against implementing inadequate intervention materials (Whittingham, 2008a; Whitthingham, 2008b).

Chapter 3 describes one of these experimental pretests. This was a pretest of materials developed for the intervention component targeting mothers' perceptions of their daughters' susceptibility towards HPV-related risks. As mentioned before, mothers' perceived daughters' susceptibility towards HPV-related risks is an important determinant of HPV vaccination acceptability (Alberts et al., 2017; Brewer \& Fazekas, 2007, Van Keulen et al., 2013a; Van Keulen et al., 2010). Two types of risk information (i.e., statistical and narrative risk information) were pretested as research remains to be mixed on the surplus value of one type over the other in influencing mothers' perceptions of their daughters' susceptibility towards HPV-related risks. The rationale behind the other two experimental pretests is described in Chapter 4, together with the results from both these pretests and the focus groups. These were conducted in order to adapt the intervention to the requirements and preferences of the mothers, which maximized compatibility with their needs. 


\section{Computer-tailoring}

Computer-tailoring was the basic method for change. Tailoring is a health communication strategy by which messages are individualized to personal preferences and needs (Kreuter \& Wray, 2003). Hence, tailoring fits with the mothers' need for more personal communication about the HPV vaccination (Van Keulen et al., 2010). Besides, meta-analyses have shown that (eHealth) interventions with tailored messages are more effective than generic interventions in achieving behavioral health outcomes for a variety of behaviors in diverse populations (Krebs, Prochaska, \& Rossi, 2010; Lustria et al., 2013). The added value of tailoring is attributed to improved exposure and active information processing, more appreciation, reading, and perceived personal relevance (e.g., Brug, Oenema, \& Campbell, 2003; Ruiter et al., 2006). Since computer-tailored interventions can reach large groups of people at relatively low costs, especially when delivered via the Internet (Neville, O'Hara \& Milat, 2009), they can have a substantial impact at the population level (Noar, Benac \& Harris, 2007).

Because message tailoring has only recently been applied to vaccination, only few tailored interventions to encourage HPV vaccination have been tested (Bennett et al., 2013; Gerend et al., 2013; Grandahl et al., 2016; Hopfer, 2012). Three of these showed positive results. Hopfer (2012) found that HPV vaccination uptake doubled among participants who were exposed to a culturally tailored narrative video (i.e., a prototypical personal story) compared to controls. Gerend and colleagues (2013) found that, compared to general information, additional information tailored to the individual participant's perceived barriers increased HPV vaccination intention. Grandahl and colleagues (2016) found that an intervention delivered individually, face-to-face by school nurses positively affected beliefs towards HPV prevention as well as vaccination uptake. However, to our knowledge, effective interventions promoting HPV vaccination that can reach large groups at relatively low costs (e.g., online tailored interventions) (Neville, O’Hara, \& Milat, 2009), do not exist yet.

\section{Virtual assistants}

We planned the use of virtual assistants for delivering tailored feedback throughout the intervention. They provide opportunities for two-way interactions, which in turn fit with the mothers' need for a more interactive experience (Van Keulen et al., 2010). Research has shown that virtual assistants with human-like embodiment and voice output can provide persuasive and trustworthy feedback (Scott, Pereira, \& Oakley, 2015). Besides, it has been shown to support a person's basic psychological need for relatedness with the intervention (Bickmore \& Picard, 2005; Okun, 2008; Ryan et al., 2011). Several studies on eHealth interventions have confirmed that the presence of a virtual assistant can improve intervention effectiveness (Blanson Henkemans et al., 2009; Jin, 2010; Watson et al., 2012). 
More specifically, virtual assistants improve recall of information compared to a text and picture-based website (Beun, de Vos \& Witteman, 2003), transfer of learning (Atkinson, 2002), the amount of learning (Baylor \& Kim, 2004), self-efficacy expectations, literacy and behavior change (Blanson Henkemans et al., 2009; Blanson Henkemans et al., 2008; Jin, 2010). And the mere presence of such an animated interface agent has a positive effect on experiencing fun and engagement (Baylor, 2009; Jin, 2010; Wik \& Hjalmarsson, 2009). The main purpose of the virtual assistants was to provide mothers with social support, which is an important factor associated with positive health outcomes in general (Bickmore et al., 2010).

\section{PART III: INTERVENTION EVALUATION}

Evaluation of an intervention is vital to determine whether it works and to help refine intervention delivery. Evaluation will not only provide feedback on the effectiveness of an intervention but will also help to determine whether the intervention is appropriate for the target population and whether there are any problems with its implementation. To evaluate the intervention, we conducted both an effect and process evaluation.

\section{Effect evaluation}

Effect evaluation describes the difference in outcomes with and without the intervention. It can be described as either efficacy or effectiveness (Bartholomew Eldredge et al., 2016). Efficacy refers to a program evaluated under optimal conditions (Singal, Higgins, \& Waljee, 2014). Effectiveness means a program evaluated under real-world circumstances (Singal, Higgins, \& Waljee, 2014). To assess both efficacy and effectiveness, we conducted a randomized controlled trial (RCT). The outcomes of the RCT had already been specified at the start of intervention development (i.e., HPV vaccination uptake, informed decisionmaking, decisional conflict and determinants of HPV vaccination acceptability). Results of the effect evaluation are presented in Chapter 5.

\section{Process evaluation}

Process evaluation provides insights into why an intervention is successful or not (Moore et al., 2014). These insights, in turn, can be used to interpret the outcomes of the effect evaluation (Grimshaw et al., 2007). Also, process evaluation helps optimizing future interventions and applying similar interventions in different settings. Process evaluation can be used to "assess fidelity and quality of implementation, clarify causal mechanisms and identify contextual factors associated with variation in outcomes" (Craig et al., 2008, p. 337). Fidelity of the implementation refers to the degree to which an intervention is delivered as intended. One of the main factors that influences implementation fidelity, is 
program adherence (Carroll et al., 2007). However, limited use of interventions is a known problem within the eHealth field (Eysenbach, 2005). Limited use hampers the evaluation of intervention effects and must be considered when determining whether the intervention is effective or not. Therefore, in the RCT, we also evaluated intervention feasibility and program adherence. Results of the process evaluation are described in Chapter $\mathbf{5}$.

\section{Dissertation outline}

The studies that have been conducted in this $\mathrm{PhD}$ project were aimed at systematically developing and evaluating an interactive, Web-based, tailored intervention with virtual assistants promoting HPV vaccination acceptability among mothers of invited girls. Chapter 2 describes a longitudinal study which was about verifying the extent to which the determinants found in earlier cross-sectional studies predict future HPV vaccination uptake. Chapter 3 describes an experimental pretest of intervention materials targeting mothers' perceived daughters' susceptibility towards HPV related risks. Chapter 4 provides a systematic description of the design rationale behind the intervention. Chapter $\mathbf{5}$ describes the effects of the intervention on HPV vaccination uptake, informed decision-making, decisional conflict and determinants of HPV vaccination acceptability. Finally, Chapter 6 discusses the major findings of the studies, critically reflects on these findings and discusses implications for future research and practice. 



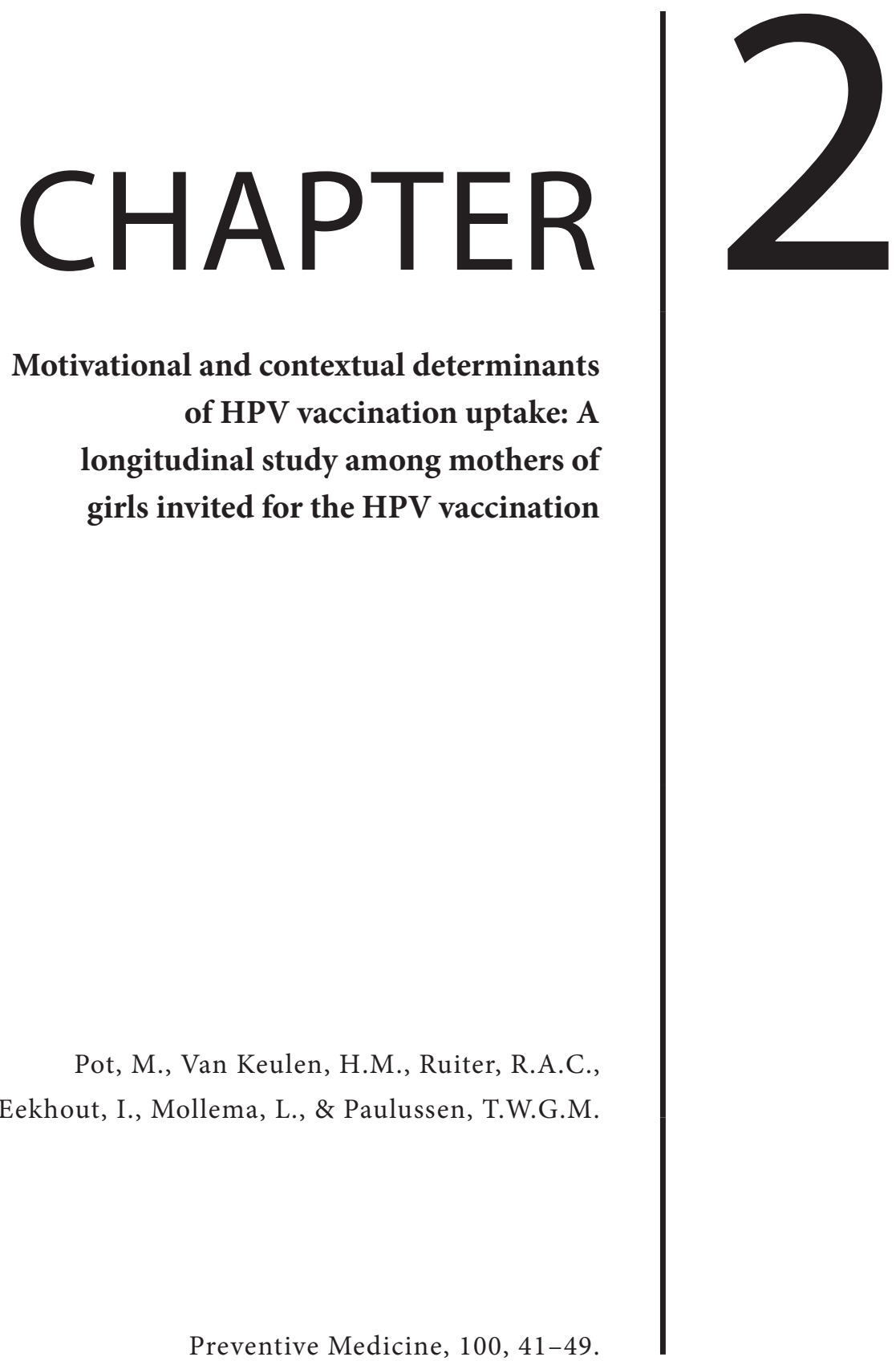




\section{ABSTRACT}

In the Netherlands, HPV vaccination uptake among 12-year-old girls remains to be lower (61\% in 2016) than expected. The present study is about (1) replicating the extent to which social-psychological determinants found in earlier cross-sectional studies explain HPV vaccination intention, and (2) testing whether HPV vaccination intention, as well as other social-psychological determinants, are good predictors of future HPV vaccination uptake in a longitudinal design. A random sample of mothers of girls invited for the vaccination in 2015 was drawn from the Dutch vaccination register (Praeventis) $(\mathrm{N}=36,000)$ and from three online panels $(\mathrm{N}=2,483)$. Two months prior to the vaccination of girls, their mothers were requested to complete a Web-based questionnaire by letter (Praeventis sample) or by e-mail (panel samples). HPV vaccination uptake was derived from Praeventis. Backward linear and logistic regression analyses were conducted to examine most dominant predictors of HPV vaccination intention and uptake, respectively. The total sample used for data analyses consisted of 8,062 mothers. Response rates were $18 \%$ for the Praeventis sample and $47 \%$ for the panel samples. HPV vaccination intention was best explained by attitude, beliefs, subjective norms, habit, and perceived relative effectiveness of the vaccination; they explained $83 \%$ of the variance in HPV vaccination intention. Intention appeared to be the only stable predictor of HPV vaccination uptake and explained $43 \%$ of the variance in HPV vaccination uptake. These results confirm what was found by earlier cross-sectional studies, and provide strong leads for selecting relevant targets in the planning of future communication strategies aiming to improve HPV vaccination uptake. 


\section{INTRODUCTION}

Worldwide, cervical cancer is the third most common cancer among women (Ferlay et al., 2010). Persistent infection by the human papillomavirus (HPV) appears the major cause of cervical cancer (Schiffman \& Castle, 2003). In the Netherlands, yearly 600 new cases of cervical cancer are detected, of which 200 with fatal consequences (Gezondheidsraad, 2008), despite the national cervical cancer screening program for women aged 30-60 years (Braspenning et al., 2001). Therefore, since 2010, the HPV vaccination of 12-year-old girls was included in the National Immunization Program (NIP). Beforehand, HPV vaccination uptake was expected to be lower compared to the uptake rate usually found for other childhood vaccinations in the Netherlands (around 95\%), because the vaccine was new, targeting a sexual transmitted disease, and it was offered to a new age group and only to girls (Rondy et al., 2010). But, so far, HPV vaccination uptake turned out to be even lower (61\% in 2016) (van Lier et al., 2016) than was expected beforehand (70\%). Earlier crosssectional research (Van Keulen et al., 2013a; Van Keulen et al., 2013b) in the first two years after initial implementation of the HPV vaccination showed mothers to be most important in the process of decision-making about their daughters' HPV vaccination. Attitude, outcome beliefs, subjective norms, habit strength, and decisional conflict were associated with significant proportions (82-94\%) of the variance in the mothers' intention towards the HPV vaccination of their daughter (Van Keulen et al., 2013b).

The aim of this study is to provide stronger leads for future education about the HPV vaccination by (1) replicating the extent to which the social-psychological determinants found in earlier cross-sectional studies explain HPV vaccination intention (Van Keulen et al., 2013a; Van Keulen et al., 2013b), and (2) longitudinally testing whether HPV vaccination intention, as well as other social-psychological determinants, are good predictors of future HPV vaccination uptake. We applied a briefer version of the questionnaire used by these former cross-sectional studies (Van Keulen et al., 2013a; Van Keulen et al., 2013b), to reduce the time burden for completing the questionnaire. We removed determinants that appeared non-significant in these former studies (e.g., opinions about alternative medicine) (Van Keulen et al., 2013a).

\section{METHODS}

\section{Participants and procedure}

The study was approved by the METC, the ethical committee of the VU Medical Center in Amsterdam. The study was part of a planned evaluation of an innovative Web-based intervention targeting mothers of girls-to-be-invited for the HPV vaccination in 2015. 
Questionnaire data collected at baseline (January 2015, before the HPV vaccination round) accounted for the social-psychological determinants and HPV vaccination intentions. After completing that vaccination round, data about girls' actual HPV vaccination uptake were derived from Praeventis. Because invited girls were given the opportunity to catch-up their missed HPV vaccination, complete data on uptake came available 18 months after baseline (i.e., July 2016). We randomly recruited mothers from three internet panels (i.e., Veldkamp BV, Intromart GFK and NGO Flycatcher, N = 2,483) in an attempt to guarantee a suitable subsample for the planned efficacy trial (Van Keulen et al., 2013a). These panels can be used for nationally representative surveys and surveys among subgroups. Panel members were recruited based on information about their characteristics, that is, being a mother of a 12-year-old girl. Panel members were pre-stratified by region for geographic diversity. In addition, we randomly recruited mothers of girls to-be-invited for the HPV vaccination from Praeventis $(\mathrm{N}=36,000)$. Praeventis is the Dutch electronic vaccination register which monitors the vaccination status for all children up to 18 years living in the Netherlands. Using this system, children are invited for receiving vaccinations to the NIP (Van Lier et al., 2016). This subsample enabled us to anticipate the naturalistic condition for future implementation of the intervention, which provided the opportunity for testing the intervention's effectiveness. A flow diagram of the recruitment and response is shown in Figure 2.1. In total, 537 participants were excluded, since they did not meet the inclusion criteria (i.e., female, aged 24-62 years, and having a daughter born in 2002). We also excluded participants that were found to be duplicates across the two samples $(n=3)$. The final response rate was $19 \%(n=6,918)$ in the Praeventis-sample and $47 \%(n=1,144)$ in the panel-sample. The final sample for data analyses consisted of 8,062 mothers.

Participants received an invitational letter by mail (Praeventis-sample) or email (panelsample), which included information about the study, a link to a secured website, and a unique code for entrance to the survey. On the website, participants were assured of their privacy, the confidentiality and security of handling their responses, and were informed that they could withdraw from participation at any time. Participants were then asked to provide informed consent and to give permission to have their daughters' HPV vaccination status requested from Praeventis. A reminder was sent one week after the first invitation. The questionnaire was open for response January $17^{\text {th }}-29^{\text {th }} 2015$. 


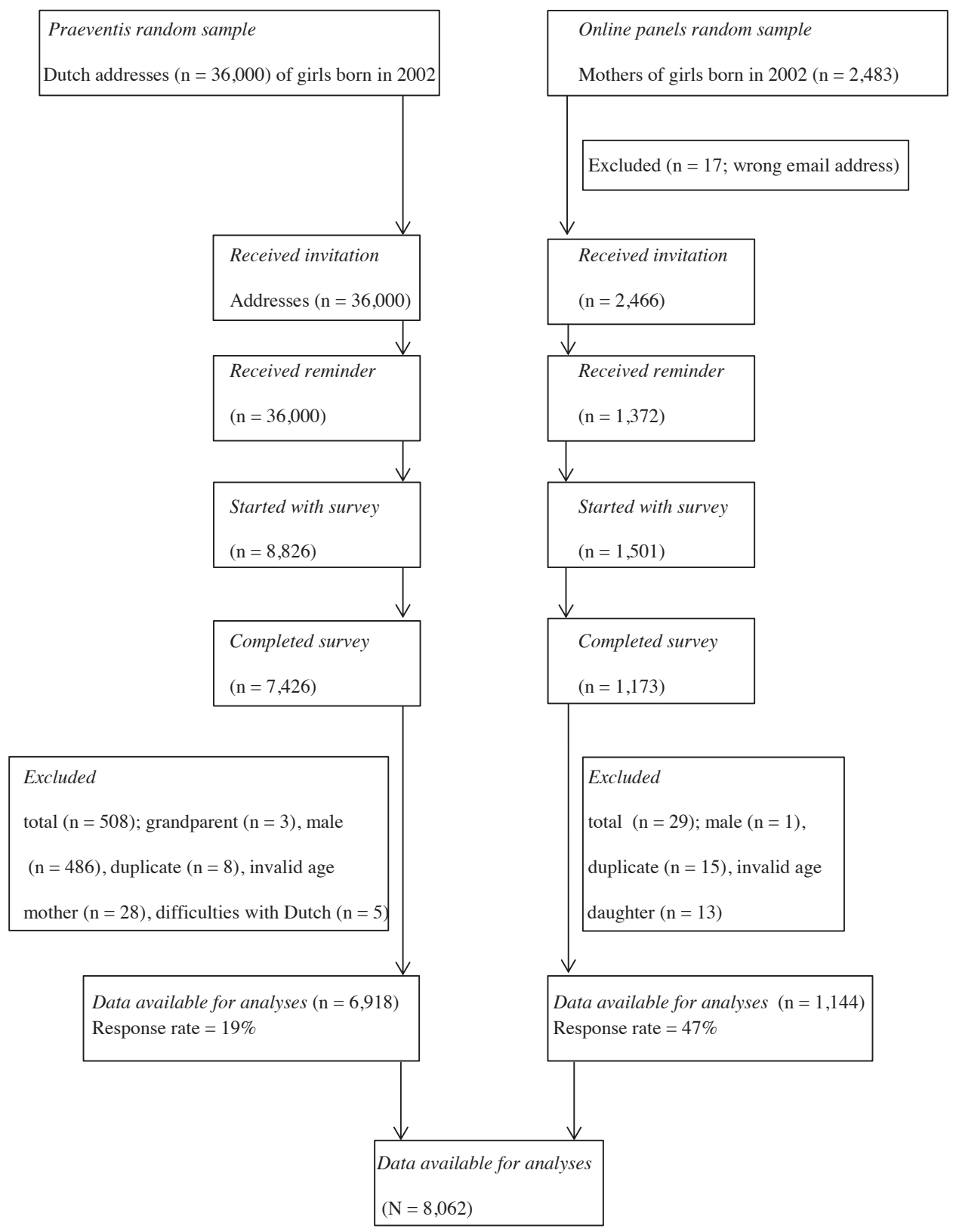

Figure 2.1 Flow diagram of the recruitment and response of study participants. ${ }^{*}$ Participants could be excluded based on multiple criteria (e.g., a male with an invalid age). Therefore, the total amount of Praeventis participants excluded differed from the sum of separate criteria for exclusion. This study has been conducted in the Netherlands, between January 2015 - July 2016. 


\section{The questionnaire}

Mothers who consented to participate were asked to complete a Web-based questionnaire. The empirical findings of Van Keulen and colleagues (Van Keulen et al., 2013a; Van Keulen et al., 2013b), the Theory of Planned Behaviour (Ajzen, 1991), Social Cognitive theory (Bandura, 1986) and the Health Belief Model (Becker, 1974) led to designing this questionnaire. The questionnaire was pre-tested and subsequently revised.

$H P V$ vaccination uptake was requested from Praeventis, which was registered as having received no, one or two injections. HPV vaccination uptake was dichotomized into having received no HPV injection ( $0=$ not vaccinated $)$ versus having received one or two HPV injections ( 1 = vaccinated), as data-analyses indicated the largest differences on determinants between these two groups.

Social-psychological determinants accounted for were intention, attitude, risk perception, beliefs, anticipated regret, relative effectiveness of the HPV vaccination compared to alternative methods, subjective norms, habit, and decisional conflict. Measurement details can be found in Table 2.1. Modifications that were made to the questionnaire used by the former study (Van Keulen et al., 2013a) concerned the assessments of intention, knowledge, subjective norms and self-efficacy, as described below.

$H P V$ vaccination intention was measured by two new items: 'are you planning on getting your daughter vaccinated against HPV?' and 'how big is the chance that you will get your daughter vaccinated?' ( 1 = definitely not / very low to 7 = definitely / very high). This was done because mothers in the former study had already made a decision about the HPV vaccination, while in the present study, they had not.

The number of subjective norms items were reduced by only including the partner and daughter as social referents.

Self-efficacy was measured by two items from Van Keulen et al., (2013a; 'having a good talk with my daughter about the HPV vaccination' and 'having a good talk with my partner about the HPV vaccination') (Van Keulen et al., 2013a), and three new items: 'guiding my daughter in the decision regarding the HPV vaccination', 'motivating my daughter to have herself vaccinated' and 'getting the actual HPV vaccination with my daughter'. 


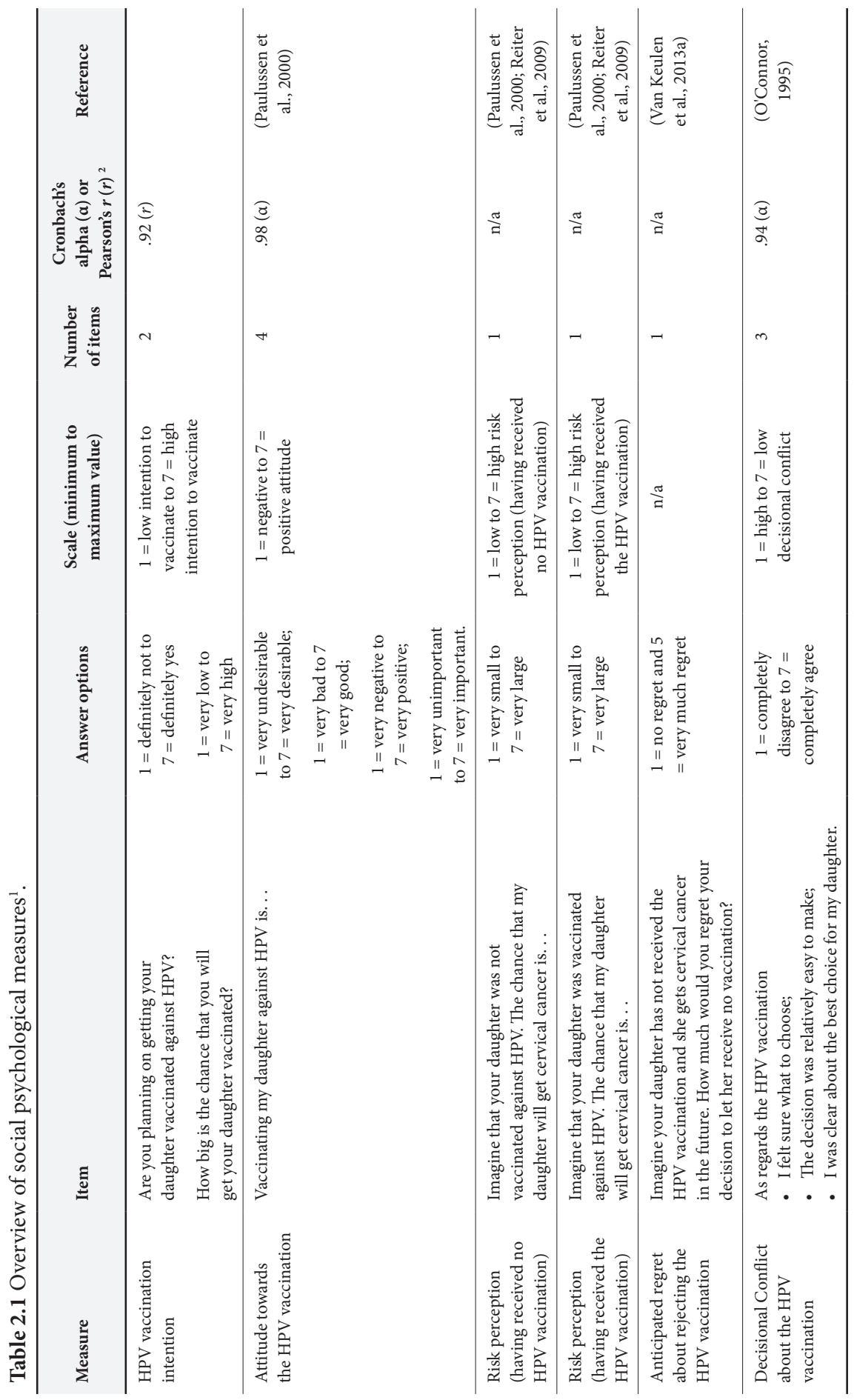




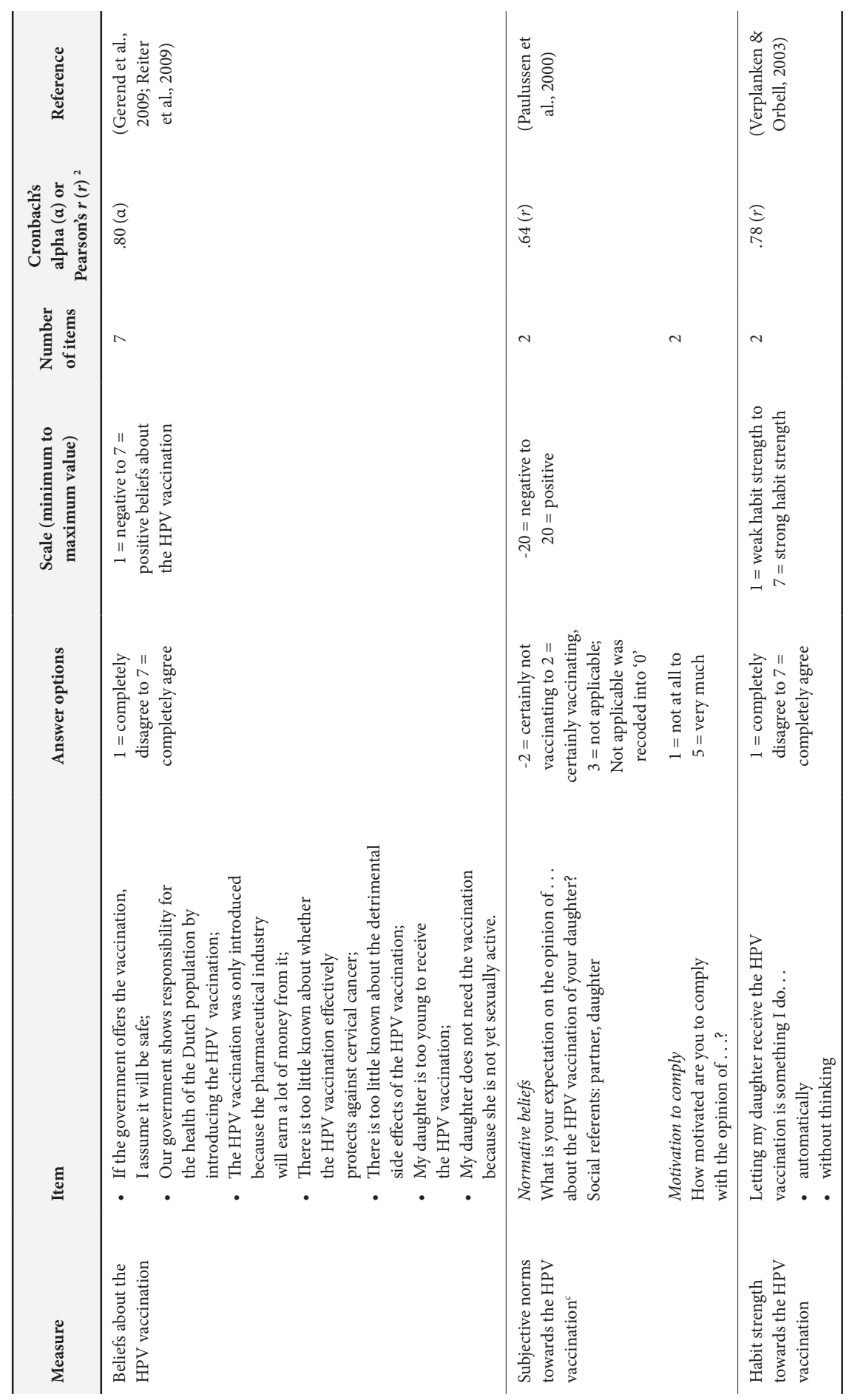




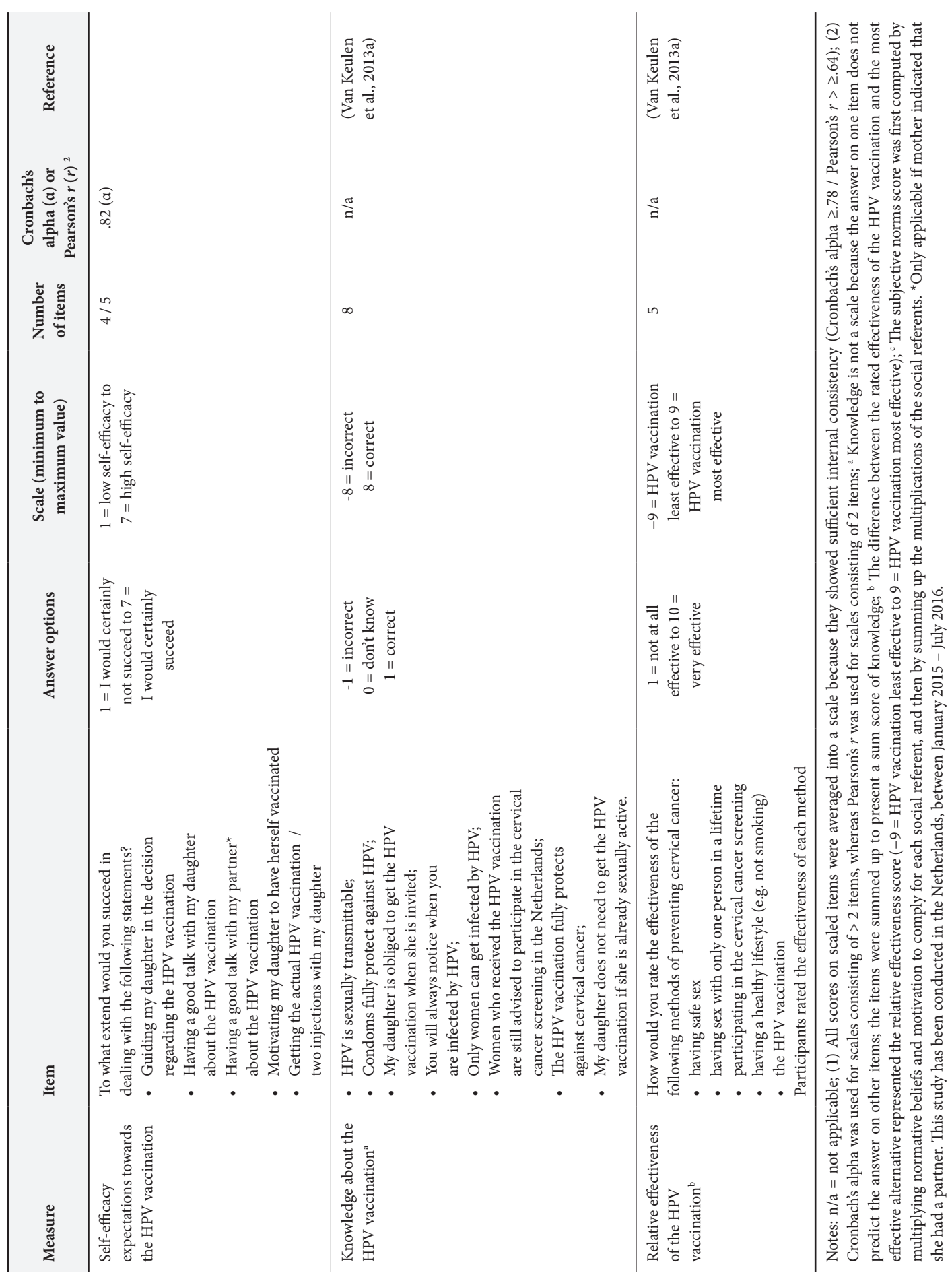


Knowledge was measured by eight items, of which two items from the former study ('HPV is a virus' and 'the HPV vaccination in the Netherlands consists of three injections') were replaced by the items 'condoms fully protect against HPV' and 'my daughter does not need to get the HPV vaccination if she is already sexually active' $(-1=$ incorrect, $0=$ don't know, $1=$ correct $)$.

Socio-demographics were modeled as background variables: age, educational level, country of birth, and religion.

Level of education was measured by asking mothers about the highest completed level of education; it was classified as low (less than secondary or vocational education), intermediate (secondary through pre-university education) or high (professional or university education) (Van Keulen et al., 2013a).

Country of birth was dichotomized into 'Netherlands' versus 'other' because the percentage of participants in the latter category was too small (7\%) for further subdivision.

Religion was measured by asking mothers about their religious convictions Religion was dichotomized into 'Protestant' (i.e., Protestant religion) versus 'not Protestant' (i.e., Roman Catholic, Muslim, Jewish, Buddhist, Hindu, other, or no religion), because data-analyses and earlier research showed that more people with a Protestant religion refrain from vaccination compared to the other groups (Van Keulen et al., 2013a).

\section{Data analyses}

Statistical Package for the Social Sciences (SPSS; version 22) was used for analyzing the data (IBM Corp., 2013). First, we examined the association between social-psychological determinants and HPV vaccination intention by using univariate and multivariate regression analyses. Social-psychological determinants that appeared statistically significant in the univariate analyses $(p<.003$; Bonferroni corrected alpha $=.05 / 17)$ were included in the multivariate linear regression analyses to assess the total amount of variance explained in HPV vaccination intention, and to explore the unique proportion of variance explained by each step in the final regression model (1) social-psychological determinants; (2) sociodemographics; (3) sample background). Manual backward selection was used to exclude non-significant variables $(p>.003$; Bonferroni correction alpha $=.05 / 17)$ except for sociodemographics and sample background because we wanted to forcefully adjust for these determinants in the final model. This provided us with the opportunity to address potential differences between mothers with a different socio-demographic background and between the two different samples. 
Social-psychological determinants that were significantly related to HPV vaccination intention according to the multiple regression model $(p<.003$; Bonferroni correction alpha $=.05 / 16)$ were entered in a logistic regression analysis, next to intention, with HPV vaccination uptake as the outcome variable: (step 1) intention; (2) social-psychological determinants; (3) socio-demographics; (4) sample background. Again, manual backward selection was used to exclude non-significant variables $(p<.005$; Bonferroni corrected alpha $=.05 / 11)$, except for socio-demographics and sample background.

Main analyses among $50 \%$ of participants ${ }^{\mathrm{a}}$

Step I: Univariate linear regression analyses

Outcome: HPV-vaccination intention

Variables: social-psychological determinants

(e.g., attitude and anticipated regret).

Step II: Multivariate linear regression analyses
Included: significant variables Step I ${ }^{\mathrm{b}}$
Outcome: HPV-vaccination intention
Steps: (1) social-psychological determinants;
(2) socio-demographics; (3) sample
Exclusion: manual backward selection ${ }^{\text {bc }}$
Result: predictors of HPV-vaccination intention
\[ \begin{array}{l}\text { Step III: Multiple logistic regression analyses } \\ \text { Included: significant predictors final model Step II }\end{array} \]
Outcome: HPV-vaccination behavior
Steps:(1) intention; (2) social-psychological
determinants; (3) socio-demographics; (4) sample

Stability check among $25 \%$ of participants ${ }^{a}$

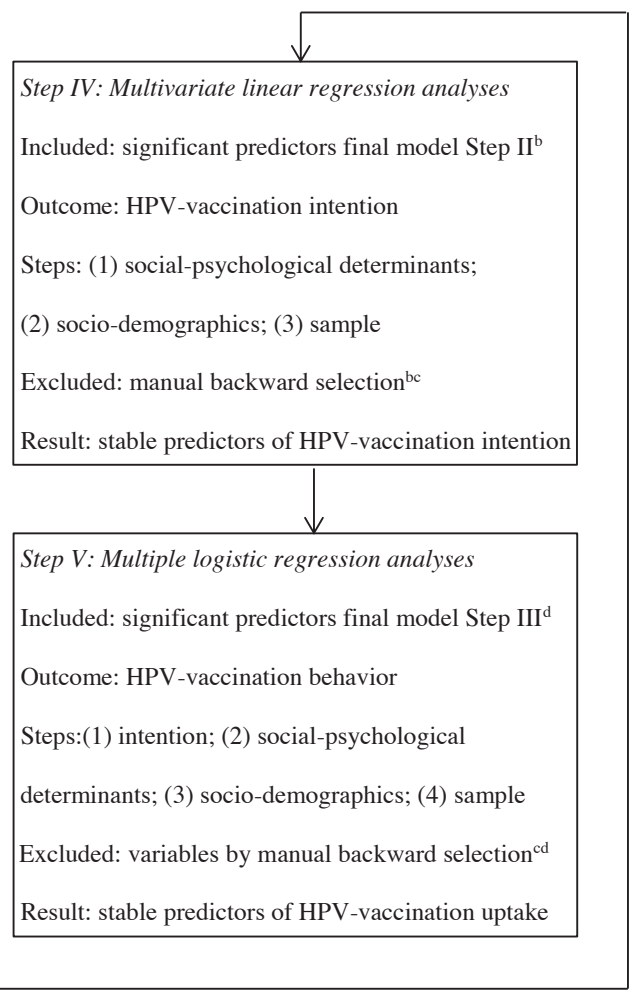

Figure 2.2 Flowchart of the stepwise nature of the main analyses and the stability check. 
Both analyses using intention and uptake as the outcome variables, were performed on a random sample of $50 \%$ of the participants. The remaining $50 \%$ of participants were randomly split in: (1) one half ( $25 \%$ of the total sample) to check for stability (i.e., predictors from the final regression model of the first 50\% sample were assumed stable when they remained significant in this $25 \%$ sample), and (2) the other half (the last $25 \%$ of the total sample) to check for generalizability. See Figure 2.2 for a flowchart of the stepwise nature of the main analyses and the stability check. Model stability enables others to use the predictive model in different samples drawn from the same population (Palmer \& O'Connell, 2009). The generalizability check was conducted in R (R Development Core Team, 2009) and examined the predictive value of the regression models for HPV vaccination uptake in the general population. This was done to account for overestimation of the percentage of explained variance (Hastie, Tibshirani, \& Friedman, 2001). By keeping the estimated parameters (unstandardized regression coefficients) of the 50\% sample model, we estimated the goodness-of-fit of the final regression model for the second $25 \%$ sample. We then compared the percentage of explained variance between the $50 \%$ and $25 \%$ samples, large differences indicated a large amount of overfitting (R Development Core Team, 2009).

\section{RESULTS}

\section{Sample description}

The mean age of mothers was 44 years $(S D=4.25)$. Compared to the general Dutch population, the sample was overrepresented for women born in the Netherlands $(76 \%$ versus $93 \%$, respectively (CBS, 2016a), for women with a high educational level (34\% versus $43 \%$, respectively) (CBS, 2016b) and for HPV vaccination uptake of girls (61\% versus 73\%) (Van Lier et al., 2016). The sample was representative for religion (19\% being Protestant compared to $16 \%$ in the Dutch population (CBS, 2015). On average, mothers had a positive intention towards the HPV vaccination of their daughter $(M=5.35, S D=1.69)$. Mothers of whom the daughter received one or two HPV injections reported a significantly higher HPV vaccination intention than mothers of whom the daughter received no injection $(M=5.96$, $S D=1.11$, versus $M=3.70, S D=1.89$, respectively; $t(8046)=65,81 . p<.001)$. Significant differences between the two different samples were found for all socio-demographics (i.e., age, country of birth, education, and religion).

\section{Univariate and multivariate determinants of HPV vaccination intention}

All social-psychological measures were significantly $(p<.003)$ associated with intention and were therefore included in the multiple linear regression analysis in the $50 \%$ sample ( $\mathrm{n}=4,015$; Table 2.2). These social-psychological determinants (step 1) accounted for 
$83 \%$ of the variance in intention $(p<.02)$. Attitude, beliefs, decisional conflict, subjective norms, habit, anticipated regret and relative effectiveness were significantly associated with intention. Decisional conflict was removed from the final model (see Table 2.2), because the positive univariate association turned negative in the multivariate model, which might indicate a suppressor-effect. The proportion of unique variance in intention accounted for by the socio-demographic variables was small but significant (step $2 ; \Delta R^{2}=.001, p<.02$ ), whereas this was non-significant for sample background (step $3 ; \Delta R^{2}=.00, p>.02$ ).

The stability check $(n=2,015)$ revealed that anticipated regret and risk perception no longer accounted for unique variance in intention (see Table 2.2). Again, decisional conflict was removed from the final model and further analyses because we were unable to interpret the switch to a negative association in the multivariate model. Attitude, beliefs, subjective norms, habit, and relative effectiveness were confirmed as stable determinants of intention and were therefore included in the analyses with HPV vaccination uptake as the criterion. The generalizability check $(n=2,028)$, confirmed the high proportion of explained variance found in the $50 \%$ sample ( $R^{2}=83 \%$ in both samples).

\section{Multivariate prediction of HPV vaccination uptake}

Intention explained $43 \%$ of variance in HPV vaccination uptake in the $50 \%$ sample (step 1; Nagelkerke $R^{2}=.433$; Table 2.3). Both attitude and subjective norm added a small (Nagelkerke $R^{2}$ change $=.01$ ) unique proportion in uptake to what was explained by intention (step $\left.2 ; \chi^{2}(2, N=3,994)=40.18, p<.001\right)$. Also, the socio-demographics added a small (Nagelkerke $R^{2}$ change $=.007$ ) but significant proportion to the explained variance in uptake (step 3; $\left.\chi^{2}(5, N=3,994)=24.52, p<.01\right)$. There was no significant additional impact of sample background (step 4; $\chi^{2}(1, N=3,994)=4.71, p=.03$; Nagelkerke $R^{2}$ change $=.001)$. The stability check $(n=2,011)$ revealed that intention and age were the only stable predictors of HPV vaccination uptake in both the $50 \%$ and $25 \%$ sample. The generalizability check (sample $25 \% \mathrm{n}=2,028$ ) confirmed the high explained variance in the $50 \%$ sample (Nagelkerke $R^{2}=.43 \%$ in both samples). 


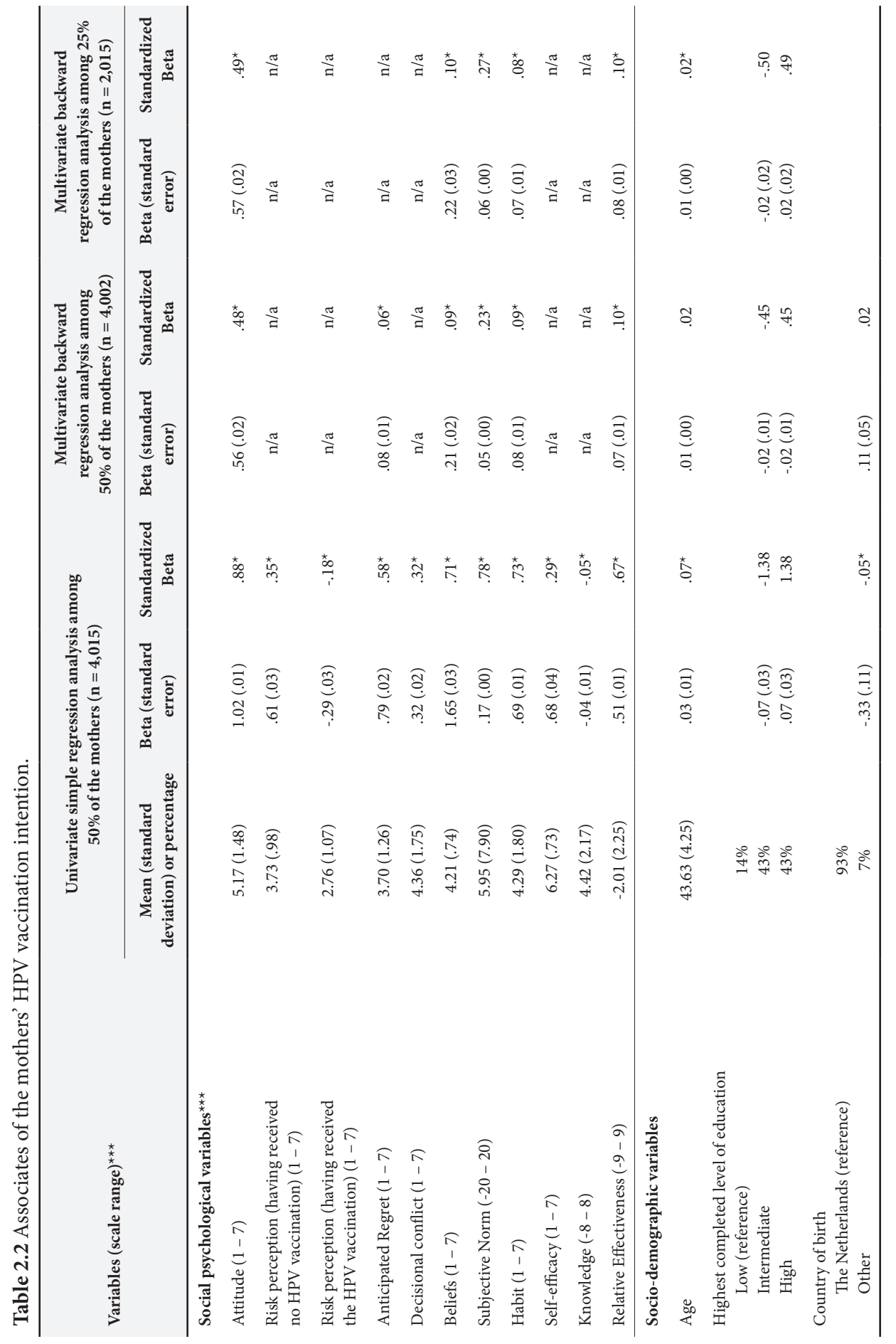




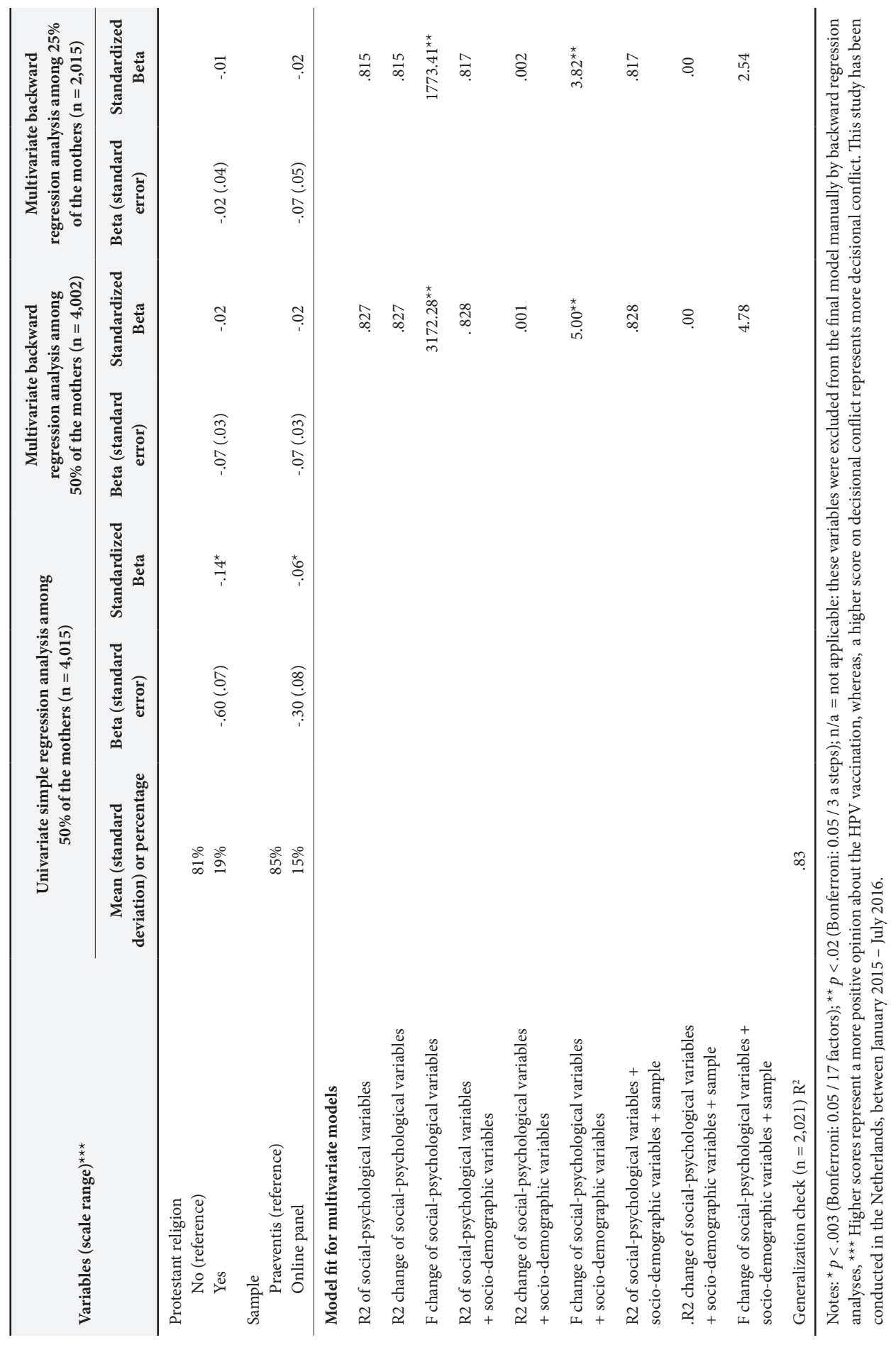


Table 2.3 Predictors of HPV vaccination uptake.

\begin{tabular}{|c|c|c|c|c|}
\hline & \multicolumn{2}{|c|}{$\begin{array}{l}50 \% \text { of the mothers }(n=3,994) \\
\qquad N_{\text {missing }}=15\end{array}$} & \multicolumn{2}{|c|}{$\begin{array}{l}25 \% \text { of the mothers }(n=2,011) \\
N_{\text {missing }}=8\end{array}$} \\
\hline & Odds ratio & 95\% C.I & Odds ratio & 95\% C.I \\
\hline Intention & $1.78^{*}$ & $1.59-1.98$ & $2.21^{*}$ & $1.97-2.48$ \\
\hline Attitude & $1.30^{*}$ & $1.14-1.47$ & $\mathrm{n} / \mathrm{a}$ & $\mathrm{n} / \mathrm{a}$ \\
\hline Subjective Norm & $1.04^{*}$ & $1.02-1.06$ & $\mathrm{n} / \mathrm{a}$ & $\mathrm{n} / \mathrm{a}$ \\
\hline Habit & $\mathrm{n} / \mathrm{a}$ & $\mathrm{n} / \mathrm{a}$ & $1.25^{*}$ & $1.13-1.38$ \\
\hline Beliefs & $\mathrm{n} / \mathrm{a}$ & $\mathrm{n} / \mathrm{a}$ & $\mathrm{n} / \mathrm{a}$ & $\mathrm{n} / \mathrm{a}$ \\
\hline Relative effectiveness & $\mathrm{n} / \mathrm{a}$ & $\mathrm{n} / \mathrm{a}$ & $\mathrm{n} / \mathrm{a}$ & $\mathrm{n} / \mathrm{a}$ \\
\hline Age & 1.03 & $1.01-1.06$ & $1.06^{*}$ & $1.03-1.10$ \\
\hline \multicolumn{5}{|l|}{$\begin{array}{l}\text { Highest completed level of education } \\
\text { Low (reference) }\end{array}$} \\
\hline Intermediate & .93 & $.84-1.02$ & .84 & $.73-.97$ \\
\hline High & 1.08 & $.98-1.19$ & 1.19 & $1.04-1.37$ \\
\hline $\begin{array}{l}\text { Country of birth } \\
\text { The Netherlands (reference) }\end{array}$ & \multicolumn{3}{|c|}{ Country of birth } & \\
\hline \multicolumn{5}{|l|}{$\begin{array}{l}\text { Protestant religion } \\
\text { No (reference) }\end{array}$} \\
\hline Yes & .74 & $.59-.92$ & .83 & $.59-1.16$ \\
\hline Sample & .76 & $.59-.97$ & .71 & $.50-1.01$ \\
\hline$\chi^{2}$ of intention $(\mathrm{df})$ & $1406.99(1)^{* *}$ & & $717.87(1)^{* *}$ & \\
\hline Nagelkerke R2 & .43 & & .44 & \\
\hline$\chi^{2}$ of social-psychological variables (df) & $40.18(2)^{\star \star}$ & & $15.10(1)^{\star *}$ & \\
\hline Nagelkerke R2 & .44 & & .45 & \\
\hline$\chi^{2}$ of social demographic (df) & $24.52(5)^{\star \star}$ & & $27.78(5)^{\star *}$ & \\
\hline Nagelkerke R2 & .45 & & .46 & \\
\hline$\chi^{2}$ of sample (df) & $4.71(1)$ & & $3.57(1)$ & \\
\hline Nagelkerke R2 & .45 & & .46 & \\
\hline $\begin{array}{l}\text { Generalizability check }(\mathrm{n}=2,019) \\
\text { Nagelkerke R2 }\end{array}$ & .43 & & & \\
\hline
\end{tabular}

Notes: ${ }^{*} p=.005$ (Bonferroni: $0.05 / 11$ variables) ${ }^{* *} p=.01$ (Bonferroni: $05 / 4$ steps); a positive OR (OR > 1) represents a higher likelihood of HPV vaccination uptake; the more positive the score, the higher the chance of the daughter being vaccinated. A negative $\mathrm{OR}(\mathrm{OR}<1)$ represents a lower likelihood of $\mathrm{HPV}$ vaccination uptake; the more negative the score, the lower the chance of the daughter being vaccinated; $\mathrm{n} / \mathrm{a}=$ backward regression analyses led to the exclusion of these determinants from the final model. This study has been conducted in the Netherlands, between January 2015 - July 2016. 


\section{Exploring the belief structure of the determinants of HPV vaccination intention}

Insight into the beliefs constituting the stable determinants of intention might be helpful for future communication about HPV vaccination. Secondary simple linear regression analyses were therefore conducted for beliefs, subjective norms, and relative effectiveness to indicate the association between each item of the scale and HPV vaccination intention. This did not account for attitude and habit since the items were too general to have relevance for future communication. All items of the constructs were moderately to strongly associated with intention ( $\beta$ 's ranged from .40 to .74, $p<.001$; Table 2.4 ). Moderately to strong associations were found between HPV vaccination intention and beliefs about (1) the responsibility of the government with regard to the HPV vaccination, (2) the daughters' sexual behaviour and age in relation to the need for the HPV vaccination, (3) the safety and effectiveness of the HPV vaccination, and (4) the role of the pharmaceutical industry in the HPV vaccination.

As for subjective norms, both the partner $(\beta=.74, p<.001)$ and daughter $(\beta=.67, p<.001)$ appeared influential. The relation between the HPV vaccination intention and the relative effectiveness of each of the alternative methods to protect against cervical cancer compared to the HPV vaccination appeared equally as strong ( $\beta$ 's ranging from .48 to $.53, p<.001$ ). The HPV vaccination was considered less effective than having safe sex, participating in the cervical cancer screening and having a healthy lifestyle. 
Table 2.4 Relationship of items of stable social-psychological determinants with the mothers' HPV vaccination intention.

\begin{tabular}{|c|c|c|c|}
\hline \multirow[b]{2}{*}{ Variabels / Items } & \multicolumn{3}{|c|}{$\begin{array}{l}\text { Univariate simple regression analyses } \\
\qquad(n=4,009)\end{array}$} \\
\hline & Mean (SD) & $\begin{array}{c}\text { Beta } \\
\text { (standard } \\
\text { error) }\end{array}$ & $\begin{array}{l}\text { Standardized } \\
\text { Beta }\end{array}$ \\
\hline \multicolumn{4}{|l|}{ Beliefs about the HPV vaccination $(1-7)^{a}$} \\
\hline If the government offers the vaccination, I assume it will be safe; & $4.87(1.63)$ & $.69(.01)$ & $.65^{*}$ \\
\hline $\begin{array}{l}\text { Our government shows responsibility for the health of the } \\
\text { Dutch population by introducing the HPV vaccination; }\end{array}$ & $5.01(1.24)$ & $.80(.02)$ & $.58^{*}$ \\
\hline $\begin{array}{l}\text { My daughter does not need the vaccination } \\
\text { because she is not yet sexually active; }\end{array}$ & $2.62(1.49)$ & $-.59(.02)$ & $-.51^{*}$ \\
\hline $\begin{array}{l}\text { There is too little known about the detrimental } \\
\text { side effects of the HPV vaccination; }\end{array}$ & $5.24(1.26)$ & $-.65(.02)$ & $-.48^{\star}$ \\
\hline $\begin{array}{l}\text { The HPV vaccination was only introduced because the } \\
\text { pharmaceutical industry will earn a lot of money from it; }\end{array}$ & $3.17(1.50)$ & $-.56(.02)$ & $-.49^{*}$ \\
\hline My daughter is too young to receive the HPV vaccination; & $3.11(1.47)$ & $-.53(.02)$ & $-.46^{*}$ \\
\hline $\begin{array}{l}\text { There is too little known about whether the HPV vaccination } \\
\text { effectively protects against cervical cancer. }\end{array}$ & $4.62(1.38)$ & $-.50(.02)$ & $-.40^{*}$ \\
\hline \multicolumn{4}{|l|}{ Subjective Norms towards the HPV vaccination $(-10 ;-10)^{b}$} \\
\hline Partner & $3.67(4.74)$ & $.27(.00)$ & $.74^{*}$ \\
\hline Daughter & $2.28(3.98)$ & $.29(.01)$ & $.67^{*}$ \\
\hline \multicolumn{4}{|l|}{ Relative Effectiveness of the HPV vaccination $(-9 ;-9)^{c}$} \\
\hline Participating in the cervical cancer screening & $-.1 .31(2.59)$ & $.35(.01)$ & $.53^{*}$ \\
\hline Having a healthy lifestyle (e.g. not smoking) & $-.23(3.02)$ & $.30(.01)$ & $.52^{*}$ \\
\hline Having sex with only one person in a lifetime & $2.04(3.57)$ & $.24(.01)$ & $.51^{*}$ \\
\hline Having safe sex & $-.20(3.35)$ & $.25(.01)$ & $.48^{*}$ \\
\hline
\end{tabular}

Notes: ${ }^{*} p<.001$; ${ }^{\mathrm{a}}$ Higher scores represent a more positive opinion about HPV vaccination; ${ }^{\mathrm{b}}$ Higher scores represent a more positive subjective norm; ${ }^{c}$ The relationship between relative effectiveness and intention was measured for each method by the association between intention and the difference between rated effectiveness of the HPV vaccination and the alternative method; higher scores indicate the HPV vaccination was rated as more effective in preventing cervical cancer than the alternative method. This study has been conducted in the Netherlands, between January 2015 - July 2016.

\section{DISCUSSION}

This study was about (a) replicating the extent to which the social-psychological determinants found in earlier cross sectional studies explain HPV vaccination intention (Van Keulen et al., 2013a; Van Keulen et al., 2013b), and (b) testing whether HPV vaccination intention, as well as other social-psychological determinants are good predictors of future uptake of girls invited for the HPV vaccination. To our knowledge, this is one of the first studies using a longitudinal study design to predict HPV vaccination uptake (Hofman et al., 2014). 
HPV vaccination uptake was best explained by intention (43\% explained variance), which was also found by Hofman and colleagues (2014). Moreover, intention was the single stable predictor of uptake, which provides support for the applicability of social cognitive models for predicting HPV vaccination uptake (e.g., the Reasoned Action Approach) (Fishbein \& Ajzen, 1975). In turn, attitude, beliefs, subjective norms, habit, and relative effectiveness explained significant unique proportions of variance in HPV vaccination intention (83\% explained variance). These findings confirm what was found in former cross-sectional studies (Van Keulen et al., 2013a; Van Keulen et al., 2013b), including the result that intention is predominantly attitude-driven, most importantly constituted in beliefs about (1) the responsibility of the government with regard to the HPV vaccination, (2) the daughters' sexual behaviour and age in relation to the need for the HPV vaccination, (3) the safety and effectiveness of the HPV vaccination, and (4) the role of the pharmaceutical industry in the HPV vaccination.

Mothers in the present study considered the HPV vaccination as less effective in preventing cervical cancer than having a healthy lifestyle, which was also found in previous research (Van Keulen et al., 2013a; Van Keulen et al., 2013b). This is surprising, because the available research has shown us otherwise (International Agency for Research on Cancer, 2005). These misperceptions (e.g., underestimating the effectiveness of the HPV vaccination while overestimating the effectiveness of having a healthy lifestyle) should be corrected in future communication. For instance, by referring to facts about the absolute versus relative effectiveness of protective methods as is indicated by various studies (Munoz et al., 2006; Schiffman et al., 2007).

The influence from both the mothers' partner and daughter (subjective norms) appeared important constituent for the mothers' HPV vaccination intention. Therefore, future communication should also target the partner and daughter. In the case of the daughter, not only because she appeared an important referent for the mother, but as valuable target in its own right since Dutch girls are formally entitled to decide themselves about the HPV vaccination.

As regards to habit, the results showed that mothers had higher intentions to let their daughters receive the HPV vaccination if they perceived this as something they did automatically, without thorough examining the pros and cons. It might be tempting to leave this situation as it is, since this relatively large group of mothers are more likely to comply to the invitation to get their daughter vaccinated. However, a less informed decision is constituted in rather instable beliefs which are susceptible to counterarguments. Nowadays, many counterarguments can be found on the internet and online social media, posted by, for instance, worried parents, anti-vaccination groups, and the alternative medical 
community (Kata, 2012). Therefore, future education should initiate active processing of verifiable information about the risks and effectiveness of the HPV vaccination in order to inoculate these mothers with arguments that become accessible in case they are confronted with (new) information that might challenge their initial positive intentions (McGuire, 1964; Paulussen et al., 2006).

The present study suggests that attitude, beliefs, subjective norms, habit, and relative effectiveness are priority targets for future education about the HPV vaccination, because these appeared stable determinants of the mothers' HPV vaccination intention. But because univariate regression analyses showed that anticipated regret, risk perception, self-efficacy, and knowledge were also associated with HPV vaccination intention, these remain relevant targets for future education as well.

The present study had some limitations. First, the total study sample did not fully represent the Dutch population. The sample was overrepresented for mothers born in the Netherlands, those having high levels of education, and those having daughters being vaccinated against HPV. A possible source of selection bias could be that access to the Internet was required for participation. However, the percentage of internet availability and usage in the Netherlands is high; $86 \%$ of individuals are daily users and $97 \%$ of households has access to the Internet (Eurostat, 2017). Therefore, we do not consider this to be a source of selection bias. Furthermore, participants from the panels could differ from a more 'naturalistic' setting as they actively chose both to be part of a panel and to participate in this study and, therefore, are self-selected. In addition, participants from the panels received a reward for their participation, whereas participants from Praeventis did not. However, although this will limit generalization of the presented mean-scores and percentages, it will hardly have obscured the tested associations, as these are less sensitive for sample selection.

Besides, the present study also had important strengths. The first is the longitudinal design, which allows us to verify the extent to which HPV vaccination intentions predict actual uptake. Also, there was a strong fit between our empirical results and theoretical assumptions in social cognitive models predicting vaccination uptake; there was a strong relationship between intention and uptake, and between intention and the social-psychological determinants. Another strength was the quality of the renewed questionnaire; it had high predictive validity $\left(R^{2}=.83\right)$ and all scales showed sufficient reliability (Cronbach's $\alpha \geq .80$ and Pearson's $r \geq .64$ ). Also, the use of registered instead of self-reported uptake had added value. 
In conclusion, this study provides a stable and generalizable model for predicting HPV vaccination uptake. HPV vaccination intention was best explained by attitude, beliefs, subjective norms, habit, and relative effectiveness of the HPV vaccination; $83 \%$ of the variance of intention was explained by these factors. In turn, intention appeared to be the only stable predictor of HPV vaccination uptake and explained $43 \%$ of the variance in uptake. This confirms earlier cross-sectional studies, and provides strong leads for selecting relevant targets in the planning of future communication strategies aiming to improve HPV vaccination uptake: Future education should (1) focus on the most relevant determinants in decision-making about the HPV vaccination (i.e., attitude, beliefs, subjective norms, habit, and relative effectiveness) and (2) target the partner and daughter because they appeared to be important social referents for the mother and because girls have their own right to know.

\section{Acknowledgements}

We would like to thank Petra Oomen (National Institute for Public Health and the Environment) for her help with data collection.

\section{Conflict of interest statement}

The authors declare that there is no conflict of interest regarding the publication of this paper. 



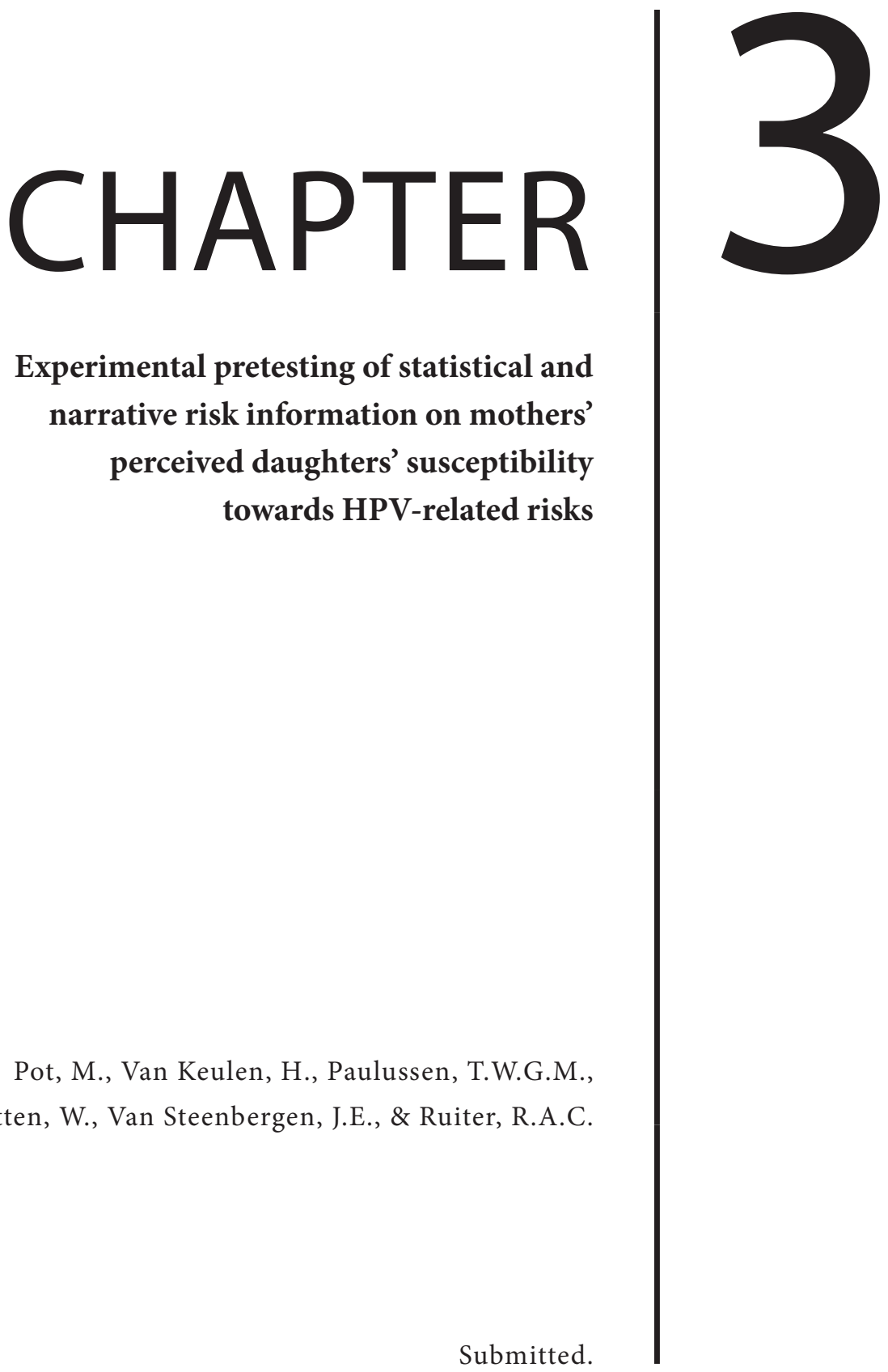




\section{ABSTRACT}

In order to promote HPV vaccination acceptability among mothers of invited girls, we developed a Web-based, tailored intervention. This study reports on an experimental pretest for the component of this intervention targeting mothers' perceived daughters' susceptibility towards HPV-related risks. Statistical versus narrative risk information was pretested as research results remain to be mixed on the surplus value of one type over the other in influencing mothers' perceived daughters' susceptibility. We used data from 375 mothers of girls-to-be invited to the HPV vaccination round 2014, who were derived from the Dutch National Immunization Register. They were randomly assigned to one of four conditions in a 2 (statistical information: yes or no) x 2 (narrative information: yes or no) between-subjects factorial design. Mothers who received statistical information felt their daughters were more susceptible towards HPV than mothers who received no statistical information. No main or interaction effects with narrative information were found. The effect of statistical information was not moderated by perceived comprehensibility, credibility or novelty of the risk information, nor was it mediated by message-induced imaginability, elaboration, personal relevance, or defensive reactions. But in secondary analyses, we found personal relevance to mediate the effect of statistical risk information on mothers' perceived daughters' susceptibility towards HPV. Statistical risk information appeared most effective in increasing mothers' perceived daughters' susceptibility towards HPV and was therefore included in the Web-based, tailored intervention promoting HPV vaccination acceptability. 


\section{INTRODUCTION}

In the Netherlands, HPV vaccination uptake remains to be low (61\%) (Van Lier et al., 2016). Hence, a need exists for evidence-based interventions aimed at increasing HPV vaccination uptake. Several studies have provided insight in factors constituting HPV vaccination acceptability (e.g., Alberts et al., 2017; Hofman et al., 2014; Pot et al., 2017b; Van Keulen et al., 2013a). Results from two Dutch studies among mothers of girls to-be-invited showed that 'modifiable' social-psychological determinants (e.g., perceived daughters' susceptibility towards HPV-related risks) accounted for large proportions of variance in HPV vaccination intention (Pot et al., 2017b; Van Keulen et al., 2013a). Mothers' intention, in turn, was found to be the main and stable predictor of their daughters' actual uptake (Pot et al., 2017b). This was also found by Hofman and colleagues (2014) among parents of invited girls and is in line with social cognitive models such as the Reasoned Action Approach (Fishbein \& Ajzen, 2010). Furthermore, mothers appeared most important in the process of decision-making about their daughters' HPV vaccination (Van Keulen et al., 2013a). These insights led to the development of an innovative, interactive, Web-based, tailored intervention promoting $\mathrm{HPV}$ vaccination acceptability among mothers of invited girls.

One of the determinants to be targeted in the intervention was perceived daughters' susceptibility towards HPV-related risks because this appeared an important determinant of mothers' HPV vaccination intention (Pot et al., 2017b; Van Keulen et al., 2013a). Besides, perceived susceptibility has been shown to be an important factor of preventive behavior according to health theory (e.g. the Health Belief Model; e.g., Schwarzer, 2001) and research (e.g., Brewer \& Fazekas, 2007). However, research findings are mixed about the surplus value of either using statistical or narrative risk information in affecting perceived susceptibility (for a review, see Winterbottom et al., 2008).

Therefore, this study is about an online experimental pretest of the effects of statistical versus narrative risk information on mothers' perceptions of their daughters' susceptibility towards HPV-related risks. Experimental pretesting enables researchers to draw reliable conclusions about the feasibility and effectiveness of developed materials in influencing predetermined objectives (Whittingham, 2008a; Whittingham, 2008b). Hence, this experimental pretest enables (a) drawing a reliable conclusion about which type of risk information is preferred when promoting mothers' perceived daughters' susceptibility towards HPV-related risks, and (b) making an evidence-based decision on which type of risk information to include in the intervention promoting HPV vaccination acceptability. 


\section{Statistical versus Narrative Risk Information}

Statistical risk information includes abstracts of numerical data and factual assertions (e.g., prevalence rates) about the probability of contracting a certain health-related outcome (De Wit et al., 2008). It is assumed that when confronted with the likelihood of contracting a risk, people are willing to take preventive actions. For instance, Cox and colleagues (2010) found that parents who received statistical information reported significantly stronger intentions to have their daughter vaccinated against HPV compared to parents who did not receive statistical information. But there is also evidence for the impact of narratives. A narrative consists of a personal experience of an event, such as somebody getting a disease (Hinyard \& Kreuter, 2007). Narratives are suggested to trigger the simulation heuristic (Kahnemann \& Tyversky, 1982). According to this heuristic, the perceived likelihood of an event is based on the ease to picture the event mentally (Gregory, Cialdini, \& Carpenter, 1982; Kahnemann \& Tyversky, 1982). Narratives are suggested to increase this ease and, therefore, are likely to increase likelihood estimates (Tversky \& Kahneman, 1974). De Wit and colleagues (2008) showed that among men who have sex with men, narrative risk information resulted in higher intentions to get the HBV vaccination than information that only mentioned an increased risk of infection. Finally, Hopfer (2012) found that when college women were exposed to a hybrid message (i.e., a message containing both statistical and narrative information) their HPV vaccination uptake was nearly double compared to when exposed to non-narrative controls (i.e., messages lacking personal decision narratives); $22 \%$ versus $12 \%$, respectively. Nan and colleagues (2015) found that undergraduate students perceived their susceptibility towards HPV to be higher after reading a hybrid message compared to narrative-only and statistics-only messages.

Despite the lack of consensus about the surplus value of statistical versus narrative risk communication, research has pointed at conditions for effectiveness (e.g., Breakwell, 2000; Priester \& Petty, 2003). Eagly and Chaiken (1993) pointed at factors moderating or mediating the effectiveness of persuasive (risk) communication. Higher comprehensibility (Eagly \& Chaiken, 1993, p. 263) and credibility (Eagly \& Chaiken, 1993, p. 352) of the message are suggested to improve message effects. And, information that is perceived as more novel, is suggested to increase the likelihood of attitude change (Wyer, 1974, p. 223). Therefore, we explored whether the effectiveness of type of risk communication is moderated by comprehensibility, credibility, and novelty of the message.

Besides, factors constituting information processing can mediate the effects of risk communication, that is imaginability, elaboration, relevance, and defensive reactions. For instance, Janssen, Van Osch, De Vries, and Lechner (2013) reported that sunbed users who were exposed to narrative information could better imagine themselves contracting skin cancer. Models of attitude change, such as the Elaboration Likelihood Model (Petty \& 
Cacioppo, 1986), state that message relevance increases active processing of information, which enhances message effects. But, a growing body of literature also suggests that messages about health risks may evoke defensive reactions such as message derogation (i.e., denying the relevance of the message or preventing it from reaching consciousness), denial of one's susceptibility (i.e., defensive avoidance), and/or perceived manipulation (Block \& Wiliams, 2002; Liberman \& Chaiken, 1992; Ruiter, Verplanken, de Cremer, \& Kok, 2004; Van't Riet \& Ruiter, 2013). Defensive reactions reduce message effects because it distracts the recipient's attention to the message (cf. Kessels, Harris, Ruiter, \& Klein, 2016). Therefore, the present study also explores whether the effects of risk information are mediated by imaginability, elaboration, relevance, and defensive reactions. See Figure 3.1 for an overview of the theoretical framework.

\begin{tabular}{|c|c|c|c|c|}
\hline $\begin{array}{c}\text { Type of risk } \\
\text { information }(\mathrm{X})\end{array}$ & $\begin{array}{c}\text { Perceived } \\
\text { susceptibility (Y) }\end{array}$ & $\begin{array}{c}\text { Attitude towards } \\
\text { the HPV- } \\
\text { vaccination }\end{array}$ & $\begin{array}{c}\text { HPV-vaccination } \\
\text { intention }\end{array}$ & $\begin{array}{c}\text { HPV-vaccination } \\
\text { uptake }\end{array}$ \\
\hline
\end{tabular}

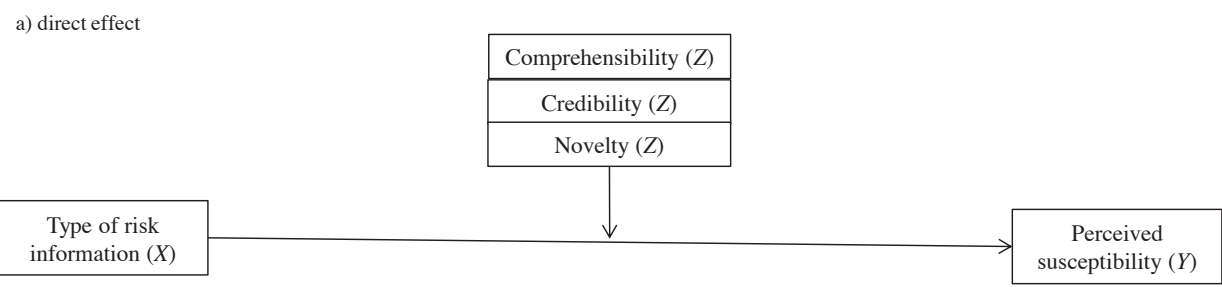

b) moderated effects

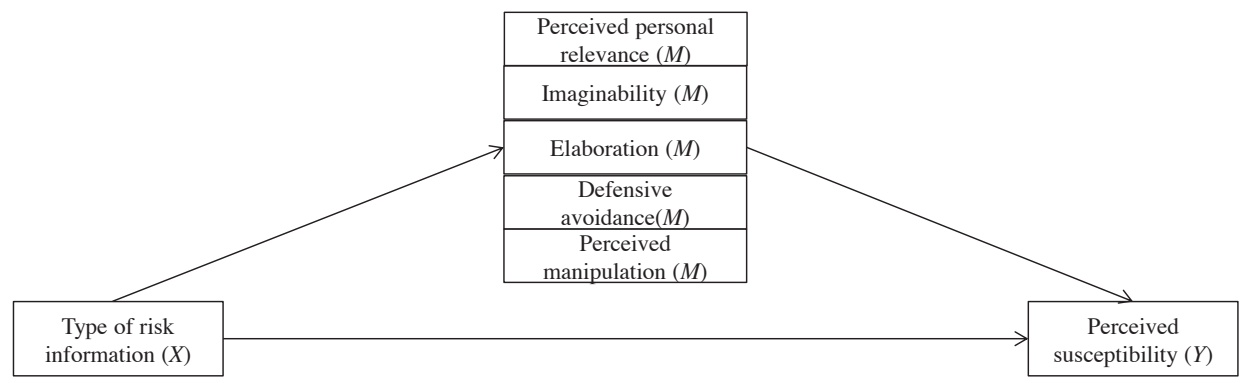

Figure 3.1 A schematic overview of the theoretical framework of perceived susceptibility in which the explorative direct, moderation and mediation effects are depicted.

Notes: The focus of this study can be found within the bold square (direct effects); variables within this study. $X=$ predictor variable; $Y=$ outcome variable; $Z=$ moderator; $M=$ mediator. 
To summarize, this study is an online experimental pretest of materials developed for an intervention promoting HPV vaccination acceptability targeting mothers of invited girls. Specifically, we aim to assess the effectiveness of statistical versus narrative risk information in influencing mothers' perceived daughters' susceptibility towards HPV and cervical cancer. To our knowledge, the effects of risk information on the latter remain to be unexplored. Another novel aspect of this study is that it explores underlying conditions and mechanisms.

\section{METHODS}

\section{Recruitment}

Participants were recruited by postal mail via Praeventis, the Dutch vaccination register. Praeventis has the name, sex, address and birth date of all Dutch children up till 18 years. A computer program was used to draw a random sample of mothers of girls born in 2001 from Praeventis (i.e., girls to-be-invited for the HPV vaccination round of 2014).

\section{Design}

Mothers were randomly assigned by the computer to one of four conditions in a 2 (statistical information: yes vs no) x 2 (narrative information: yes vs no) between-subjects factorial design.

\section{Procedure}

An invitational letter was sent to selected participants' home addresses. This included information about the study, contact details of the researchers, a link to the website, and a personal entrance code. As a reimbursement, a subscription to a magazine was raffled among eligible participants. When participants logged in to the experiment, they were first informed about the study purpose, followed by instructions for completing the experiment. After having provided informed consent, participants were assigned to one of four conditions. All participants received general information about HPV, cervical cancer, and the HPV vaccination. Next, mothers in the experimental conditions additionally received risk information (See 'manipulations'). After this, participants proceeded with self-report questionnaires that accounted for the dependent variables, proposed mediators and moderators, and socio-demographics. Finally, mothers were debriefed and thanked for their participation. On average, mothers needed 15 minutes to complete the experiment. 


\section{Required Sample Size}

Based on the literature (e.g., De Wit et al., 2008; Mevissen et al., 2009), we expected a medium effect size on primary outcomes $(\eta 2=.06$; Cohen, 1988). In our design with 2 means per factor, this effect size value corresponds to a d-value of 0.50 , and an $\mathrm{f}$-value of 0.25 . Power analysis showed that a minimal sample size of 32 participants per condition was needed (with power $=0.80$, and two-sided $=0.05$; Hintze, 2004). We took into account an attrition rate of $30 \%$ and a response rate of $5 \%$, based on previous experiences (Van Keulen et al., 2013a). Consequently, 3,680 mothers were invited.

\section{Data Collection Period}

The experimental pretest was conducted between September and October 2013.

\section{Operationalisations}

\section{Measurements}

Socio-demographics included participants' age, gender, educational level, country of birth, and religion. Level of education accounted for mothers' highest level of education they had completed. This was classified as low (less than secondary or vocational education), intermediate (secondary through pre-university education), or high (professional or university education) (Van Keulen et al., 2013a). Mothers were asked about their religious conviction (Protestant, Roman Catholic, Muslim, Jewish, Buddhist, Hindu, other, or no religion) (Van Keulen et al., 2013a). Research has shown that more Dutch Protestants refrain from vaccination compared to the other (non) religious groups (Van Keulen et al., 2013a). This was confirmed in our sample, hence religion was dichotomized into 'Protestant' versus 'not Protestant'. Country of birth was dichotomized into 'Netherlands' versus 'other' as in our sample only $6 \%$ participants were born in a country other than the Netherlands.

Criteria for effectiveness. The outcome criteria were mothers' perceived daughters' susceptibility and mothers' HPV vaccination intention. Mothers' perceived daughters' susceptibility towards HPV and towards cervical cancer was measured by 7-point scaled item, each: 'Without the HPV vaccination, the chances of my daughter for getting infected with HPV (getting cervical cancer) are ..... ' ( 1 = very small; to 7 = very big) (Pot et al., 2017b; Van Keulen et al., 2013a). HPV vaccination intention was measured as a composite of two items: 'Are you planning to have your daughter vaccinated against HPV?' and 'What are the chances that you will get your daughter vaccinated against HPV?' (Pot et al., 2017b; Van Keulen et al., 2013a). Responses were given on a 7-point scale ranging from (1) certainly not / very small / to (7) certainly / very big (Pearson's $r=.95$ ). 
Items measuring the moderating and mediating factors (except for 'defensive avoidance, see below) referred to the specific type(s) of information the mothers had received (i.e., general and/or statistical and/or narrative risk information). Thus, mothers evaluated each specific type of information separately. For mothers in the experimental conditions, a mean score per moderating and mediating factor had to be calculated. This means that, for instance, credibility for mothers in the statistical information condition represents the mean score of perceived credibility of the general ánd the statistical information.

Moderating factors. Comprehensibility was assessed by a composite of three items accounting for the extent to which mothers understood the information, and whether they considered the text to be clear and readable (Mevissen et al., 2009). Responses were given on a seven-point scale ranging from (1) not at all understandable / very unclear / very readable to (7) fully understandable / very clear / very readable (Cronbach's alpha $=.87$ ). Credibility was measured with the single-item: 'To what extent do you believe the content of the text is credible?' ( 1 = very incredible; to 7 = very credible) (Mevissen et al., 2009). Novelty was measured with a single-item: 'To what extent does the text contain new information for you?' (1 = much new information; to 7 = much known information) (Mevissen et al., 2009).

Mediating factors. Imaginability was measured with one item for HPV and one item for cervical cancer: 'Because of the text, I could imagine my daughter getting HPV / cervical cancer' ( 1 = totally disagree; to 7 = totally agree) (Broemer, 2004; Janssen et al., 2013; Mevissen et al., 2010). Elaboration was assessed by a single item 'How well did you read the information?' ( 1 = not carefully at all; to 7 = very carefully) (Chaiken, 1980). Personal relevance was measured with the item 'I considered the text to be personally relevant' ( 1 = totally disagree; to $7=$ totally agree) (Mevissen et al., 2009). Defensive avoidance was accounted for by two 7-point scaled items (one referring to HPV and the other to for cervical cancer): 'I'd rather not think about my daughter getting infected with HPV/ developing cervical cancer' ( $1=$ totally disagree; to $7=$ totally agree) (Van't Riet $\&$ Ruiter, 2013). Perceived manipulation of the information was measured by the item 'I considered the text to manipulate my beliefs' ( $1=$ totally disagree; to $7=$ totally agree) (Mevissen et al., 2009; Van't Riet et al., 2013).

\section{Manipulations}

The text messages were pretested among a sample of mothers $(\mathrm{N}=10)$ to assure comprehensibility, and, for the narrative message, to assure that mothers were able to imagine the situation. 
General information (see Appendix A). All participants received general information about HPV, cervical cancer, and the HPV vaccination, to ensure their background knowledge was similar.

Statistical risk information (see Appendix A). The statistical message was based on data from the Dutch National Institute for Public Health and Environment and data released by the Dutch Health Council (Gezondheidsraad, 2008). It presented factual epidemiological information about the prevalence of HPV and cervical cancer among women and information about the reduction in cervical cancer cases to be expected when all girls would obtain the HPV vaccination. The latter was based on a study by Cox and colleagues (2010): "when no girl gets the HPV vaccination, 600 women per year will get cervical cancer. But when all girls get the HPV vaccination, 300 women per year (half of 600) will be saved from getting cervical cancer".

Narrative risk information (see Appendix A). The narrative message was based on a personal story derived from an Internet health forum of a woman who had found out she had cervical cancer, caused years ago by an HPV-infection. It was reported in the first person, because research has shown this to be more effective in influencing perceived susceptibility than the third person (Nan et al., 2015). We changed the name of the woman into 'Anne', because it is relatively common in The Netherlands, and not specific to individuals with certain backgrounds. She was 30 years old, as it typically takes 10-20 years for an HPV infection to develop into cancer (Weinberg, 1999). It was emphasized that she got infected with HPV, despite having had an overall safe sex life (Manhart \& Koutsky, 2002; Moschicki, 2005). To ensure mothers that the message concerns their daughters' HPV vaccination, Anne ended her story by noting: "With the HPV vaccination that is available now, I sometimes can't help but wonder: 'what if I had gotten myself vaccinated against HPV 18 years ago..."

The statistical and narrative information were approximately of the same length (i.e., \pm 300 words).

\section{Analysis Plan}

A two-way analysis of variance (ANOVA) was conducted to examine the impact of the different types of risk information on perceived susceptibility and intention. Mediation and moderation analyses were examined using SPSS PROCESS macro (2.12.1 release), a widely used regression-based approach (Hayes, 2013).

For moderation analysis, the independent variable and the proposed moderator were entered in the model in the first step, followed by the interaction between the independent variable and the moderator in Step 2. The interaction (i.e., moderation) was assumed significant 
when $p<.05$. For mediation analyses, the PROCESS macro first conducted the following hierarchical regressions: the mediators $(\mathrm{M})$ were regressed onto the independent variable $(\mathrm{X})$ and the dependent variable $(\mathrm{Y})$ was regressed onto both the independent variable and the mediator. We assumed mediation when the following conditions were met: (1) X has an effect on $\mathrm{M}$, (2) M has an effect on $\mathrm{Y}$ when controlling for $\mathrm{X}$, and (3) the effect of X on $\mathrm{Y}$ becomes significantly smaller or non-significant when controlling for $\mathrm{M}$. Then, in order to test the indirect effect for significance, the bootstrapping method was utilized. The indirect effect represents the influence of the mediating variable on the original relation between $\mathrm{X}$ and $\mathrm{Y}$. A value of 0 indicates no impact. A bias-corrected 95\% bootstrap model with confidence intervals resampled 5,000 times for each analysis was used to judge significance (Shrout \& Bolger, 2002); if the bootstrap confidence interval did not include zero, mediation was supported. This method was chosen as it is considered to maximize power and is robust against non-normality (Hayes, 2013).

\section{Software}

The power calculation was conducted with PASS software (Hintze, 2004). The online experiment was developed using TailorBuilder (C) computer software. Main, moderation, and mediation analyses were conducted using SPSS version 22 (IBM Corp., 2013).

\section{Ethics}

This experiment was part of a larger study, for which ethical approval was obtained from the ethical committee of the VU Medical Center in Amsterdam.

\section{RESULTS}

\section{Sample Description}

Of the invited individuals ( $\mathrm{n}=3,680), 467$ initiated the study $(13 \%)$. Inclusion criteria were that participants had to (1) provide informed consent online (excluded: $n=1$ ), (2) fully complete the questionnaire (excluded: $n=72$ ), and (3) be female (excluded: $n=19$ ). This resulted in a final sample of $375(11 \%)$ mothers.

The mean age of mothers was 43 years $(S D=4.05)$. Almost all mothers were born in the Netherlands (94\%) and almost half of the mothers were high in education (42\%). A relatively small percentage was Protestant (16\%). 


\section{Univariate Analyses}

ANOVA revealed a significant main effect of statistical risk information on mothers' perceived daughters' susceptibility towards $\operatorname{HPV}(F(1,371)=7.56, p<.01)$. Mothers who received statistical information perceived their daughters' susceptibility towards HPV to be higher $(M=4.11, S D=.10)$ than mothers who did not receive statistical information $(M=3.73, S D=.09)$. There was no main effect of narrative risk information on perceived daughters' susceptibility towards $\operatorname{HPV}(F(1,371)=.00, p=.97)$, nor was there a significant interaction-effect between statistical and narrative information on perceived daughters' susceptibility towards $\operatorname{HPV}(F(1,371)=1.54, p=.22)$. No significant main or interaction effects were found on mothers' perceived daughters' susceptibility towards cervical cancer $\left(F^{\prime} s<2.10 ; p^{\prime} s>.15\right)$ or on HPV vaccination intention $\left(F^{\prime} s<.83 ; p^{\prime} s>36\right)$. See Tables 3.1-3.3 for the mean scores on the outcomes per condition.

Table 3.1 Mean scores $(S D)$ on susceptibility towards HPV $(1-7 ; \mathrm{N}=375)$.

\begin{tabular}{lcc}
\hline & Statistical risk information & No statistical risk information \\
\hline Narrative information & $4.02(1.28)^{\mathrm{a}, \mathrm{b}}$ & $3.82(1.23)^{\mathrm{a}, \mathrm{b}}$ \\
No narrative information & $4.19(1.43)^{\mathrm{a}}$ & $3.65(1.26)^{\mathrm{b}}$ \\
\hline
\end{tabular}

Notes: A higher score represents a higher perceived daughters' susceptibility towards HPV. Values with different superscripts differ significantly $(p<.05)$

Table 3.2 Mean scores $(S D)$ on susceptibility towards cervical cancer $(1-7 ; \mathrm{N}=375)$.

\begin{tabular}{lcc}
\hline & Statistical risk information & No statistical risk information \\
\hline Narrative information & $3.65(1.18)$ & $3.81(1.17)$ \\
No narrative information & $3.79(1.31)$ & $3.60(1.08)$ \\
\hline
\end{tabular}

Notes: A higher score represents a higher perceived daughters' susceptibility towards cervical cancer. Differences between the groups were nonsignificant.

Table 3.3 Mean scores $(S D)$ on HPV vaccination intention $(1-7 ; \mathrm{N}=375)$.

\begin{tabular}{lcc}
\hline & Statistical risk information & No statistical risk information \\
\hline Narrative information & $6.01(1.42)$ & $5.92(1.42)$ \\
No narrative information & $5.76(1.62)$ & $5.88(1.56)$ \\
\hline
\end{tabular}

Notes: A higher score represents a higher HPV vaccination intention. Differences between the groups were nonsignificant. 


\section{Moderation and Mediation Analyses}

Moderation and mediation analyses were performed using statistical risk information (yes versus no) as the independent variable and mothers' perceived daughters' susceptibility towards HPV as the dependent variable. No significant interaction effect was found with any of the proposed moderators (comprehensibility, credibility and novelty; $p$ 's $>.05$ ). Also, we didn't find any support for an indirect impact of statistical risk information on perceived daughters' susceptibility towards HPV via the proposed mediators (i.e., imaginability, elaboration, perceived personal relevance, defensive avoidance and perceived manipulation). Overall, mean scores on the moderators and mediators were high (except for defensive avoidance and perceived manipulation). Differences between the conditions were small and nonsignificant (See Table 3.4).

Table 3.4 Mean scores $(S D)$ on moderators and mediators of those who did versus those who did not receive statistical risk information $(\mathrm{N}=375)$.

\begin{tabular}{lcc}
\hline & $\begin{array}{c}\text { No Statistical Risk } \\
\text { Information }(\mathbf{n}=\mathbf{1 9 1})\end{array}$ & $\begin{array}{c}\text { Statistical Risk } \\
\text { Information }(\mathbf{n}=\mathbf{1 8 4})\end{array}$ \\
\hline Proposed moderators* & & \\
Comprehensibility $(1-7)$ & $5.83(.91)$ & $5.80(.86)$ \\
Credibility $(1-7)$ & $5.60(1.11)$ & $5.58(.99)$ \\
Novelty $(1-7)$ & $4.70(1.40)$ & $4.92(1.20)$ \\
\hline Proposed mediators* & & $5.74(.81)$ \\
Imaginability HPV $(1-7)$ & $5.76(.91)$ & $5.63(.90)$ \\
Imaginability cervical cancer $(1-7)$ & $5.59(1.04)$ & $5.86(.84)$ \\
Elaboration $(1-7)$ & $5.93(.97)$ & $5.42(1.04)$ \\
Perceived personal relevance $(1-7)$ & $5.24(1.10)$ & $3.42(2.00)$ \\
Defensive avoidance HPV $(1-7)$ & $3.65(2.02)$ & $3.64(2.10)$ \\
Defensive avoidance cervical cancer $(1-7)$ & $3.68(2.06)$ & $4.09(1.50)$ \\
Perceived manipulation $(1-7)$ & $3.70(1.64)$ & \\
\hline
\end{tabular}

Notes: ${ }^{*} \mathrm{~A}$ higher score represents a higher score on the moderator / mediator. Differences between the groups were nonsignificant.

\section{DISCUSSION}

This study was an experimental pretest of materials targeting mothers of invited girls developed for a Web-based, tailored intervention promoting HPV vaccination acceptability. We tested the effectiveness of statistical versus narrative risk information on affecting how mothers' perceived their daughters' susceptibility towards HPV and cervical cancer. Results 
showed that statistical information increased mothers' perceptions of their daughters' susceptibility towards HPV. Such a positive effect was also found by Mevissen and colleagues (2009), within the context of Chlamydia: participants felt more susceptible to a Chlamydia infection after they read statistical risk information compared to those exposed to narrative or no risk information. These results made us decide to include statistical information as the mode of communication about HPV-related risks in the Web-based intervention.

We did not find statistical risk information to affect mothers' perceptions of their daughters' susceptibility towards getting cervical cancer. This might have been caused by the actual difference in the likelihood of contracting HPV (high probability), relative to contracting cervical cancer (low probability). In the present study, we found that mothers who received no risk information highly overestimated the probability of their daughter contracting cervical cancer when looking at the actual incidence in the Netherlands (Integraal Kankercentrum Nederland, 2013). It seems unlikely that exposure to statistical risk information -presenting the actual low probability rate- would increase mothers' perceptions of their daughters' susceptibility for contracting cervical cancer.

Mothers who received no risk information perceived the chance of their daughter contracting HPV or cervical cancer to be almost similar, while in fact, the chance of contracting HPV is much higher (Integraal Kankercentrum Nederland, 2013). So, it seemed that mothers underestimated the risk for HPV, and overestimated the risk for cervical cancer. The tendency to overestimate small frequencies and underestimate larger ones, is well known (Fischhoff, Bostrom \& Quadrell, 1993; Lichtenstein et al., 1978). Correcting mothers' misperceptions about the risks of their daughter contracting HPV and cervical cancer, would provide a more realistic and bases for initiating processes of informed decision-making.

In this study, narrative risk information had no effect on how mothers perceived their daughters' susceptibility towards HPV or cervical cancer. We hypothesized narratives to have an effect by increasing the ease of imagination (Kahnemann \& Tyversky, 1982). But secondary analysis indicated that mothers could imagine their daughter contracting both HPV and cervical cancer less easily after reading the narrative compared to the statistics. The absence of an effect of narrative information may also have been caused by difficulty for mothers to identify their adolescent daughter with the 30 -year-old woman getting cervical cancer. Identification has been shown to be an important effect condition for narratives (Cohen, 2001; de Graaf et al., 2012). Difficulties with mothers' identification of their daughter may also have been caused by the relatively young age at which the character had sex for the first time (i.e., 16 years old). In 2017, 28\% of Dutch girls aged 15-17 has had sex for the first time, compared to $67 \%$ of girls aged 18-20 (De Graaf et al., 2017). Finally, mothers may not have been 'transported' into the narrative enough. Transportation into narrative worlds, or 
immersion into a story, is a primary mechanism of narrative persuasion (Green \& Brock, 2000). Unfortunately, we did not assess the degree of mothers' identification of the daughter with the story's character nor did we measure the extent of transportation into the narrative.

Although mothers who received statistical information felt their daughters were more susceptible towards HPV, this did not result in a higher HPV vaccination intention. An explanation comes from social cognitive models of health behavior (e.g., the Health Belief Model; e.g., Schwarzer, 2001) and earlier research (Pot et al., 2017b; Van Keulen et al. 2013a), which indicate that perceived daughters' susceptibility is not the sole determinant of mothers' intention to have their daughter vaccinated against HPV. Thus, to influence intention, communication should as well target other relevant determinants of HPV vaccination uptake (e.g., attitudinal beliefs) (Pot et al., 2017b; Van Keulen et al., 2013a). In the Web-based intervention, other relevant determinants of HPV vaccination uptake were also targeted.

The effect of statistical risk information on mothers' perceptions of their daughters' susceptibility towards HPV was not moderated by message characteristics (i.e., comprehensibility, credibility and novelty), nor was it mediated by factors constituting information processing (i.e., imaginability, elaboration, perceived personal relevance, and defensive reactions). This indicates that, in this study, statistical risk information increased mothers' perceptions of their daughters' susceptibility towards HPV irrespective of the information being believed as more or less comprehensible, credible or novel. And, the increase in mothers' perceptions of their daughters' susceptibility towards HPV could not be explained by how mothers processed the statistical information (e.g., by being perceived as more imaginable). This absence of effects may be due to the fact that we were forced to compute a mean for moderating and mediating factors when mothers received more than one type of information. Thus, for the combination condition, the evaluations of three pieces of risk information (i.e., the general, statistical and narrative information) were averaged. Moderation and mediation was tested between the subgroups 'statistical risk information' (i.e., statistical and combination condition) and 'no statistical risk information' (i.e., no risk information and narrative risk information condition). It could well be that actual differences between the statistical versus narrative information were diluted because of this averaging over the separate pieces of risk information. An indication for this comes from secondary analyses, in which we only compared the statistical $(\mathrm{n}=102)$ and narrative condition (n = 89) (i.e., the general and combination condition were excluded) on the moderator and mediator evaluations of the experimental information only (i.e., the evaluations of the general information was excluded). This way, the scores for moderating and mediating factors did not have to be averaged. Mean scores on moderating and mediating factors differed significantly between the two conditions for the moderator comprehensibility (i.e., 
mothers understood the narrative better) and for the mediators imaginability (i.e., mothers could imagine their daughter contracting both HPV and cervical cancer better after reading statistics) and personal relevance (i.e., mothers judged the statistics as more relevant). In addition, we found personal relevance to mediate the effect of statistical information on mothers' perceived daughters' susceptibility towards HPV: statistical information was judged more personal relevant, which increased mothers' perceived daughters' susceptibility towards HPV. This is in line with the Elaboration Likelihood Model (Petty \& Cacioppo, 1986), which suggests that message relevance increases active processing of messages, which in turn enhances message effectiveness if persuasive arguments are perceived as strong. Moreover, this implicates that personal relevance is a condition for statistical risk information to be effective. Hence, statistical messages should be designed in a way that is personally relevant to the message recipient. This can be done, for instance, by tailoring the message to the recipient, as tailored messages have shown to improve personal relevance (Kreuter \& Wray, 2003).

\section{Limitations}

This study had several limitations worth mentioning. First, we did not include identification nor transportation as mediators, which could have helped in disentangling why the narrative used in this study was not effective, since these factors have been shown to be important effect conditions for narratives (Cohen et al., 2001; de Graaf et al., 2017). Second, we cannot exclude that the general information affected mothers' perceived daughters' susceptibility. However, general information is an essential element in communication about the HPV vaccination, as sufficient knowledge is a prerequisite for an informed decision (Marteau, Dormandy \& Michie 2001). Third, the use of single-item measures represents a limitation. These are suggested to be less stable, reliable, and precise compared to multi-item measures (Bowling, 2005). The questionnaire used for this experiment included additional questions about design features of the developed intervention. In order to reduce the time burden for participants, single-items were used. Finally, response rate was low (11\%). Although it was higher than found in a previous study which recruited mothers through Praeventis (Van Keulen et al., 2013a), the low response rate could be explained by the use of a written invitation letter. Research has shown written invitations to result in lower response rates compared to e-mail invitations in studies using online questionnaires (Manfreda et al., 2008). Due to the low response rate, caution is needed in generalizing the study results.

\section{Recommendations for Future Research}

We recommend future research to investigate how to best present statistical risk information to maximize its effectiveness. For instance, Cox and colleagues (2010) found that parents who viewed a graphic presentation of statistical information had a higher HPV vaccination 
intention compared to parents who viewed a nongraphic presentation. Finally, we recommend future research to investigate how to make narratives about HPV-related risks more effective, as we still believe narratives to be a promising method in communicating about HPV-related risks (e.g., De Wit et al. 2008; Janssen et al., 2013; Mevissen et al., 2012). To improve effectiveness, future research could use strategies such as multiple narrative messages (Mevissen et al., 2010), self-construed narratives (Mevissen et al., 2012), and tailoring the narrative to the recipients characteristics (Kreuter et al., 2007).

\section{Take Home Messages}

Statistical risk information was most effective in increasing mothers' perceived daughters' susceptibility towards HPV and was therefore implemented in the Web-based, tailored intervention promoting HPV vaccination acceptability. Future research is needed on (a) how to best present statistical risk information and (b) how to make narratives about HPVrelated risks more effective.

\section{Acknowledgements}

We would like to thank Petra Oomen (RIVM) for her help with data collection.

\section{Conflict of interest statement}

The authors declare that there is no conflict of interest regarding the publication of this paper. 




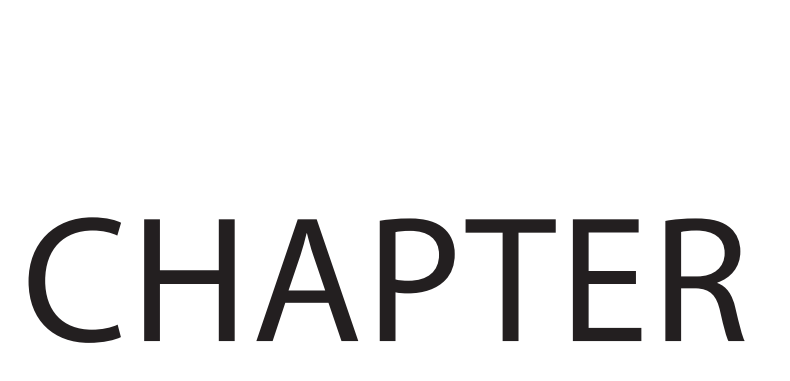

Systematically Developing a Web-based Tailored Intervention Promoting HPV Vaccination Acceptability among Mothers of Invited Girls using Intervention Mapping

Pot, M., Ruiter, R.A.C., Paulussen, T.W.G.M., Heuvelink, A., de Melker, H. E., Van Vliet, H.J.A., \& Van Keulen, H.M.

Submitted. 


\section{ABSTRACT}

Currently, the eHealth field calls for detailed descriptions of theory-based interventions in order to support improved design of such interventions. This article aims to provide a systematic description of the design rationale behind an interactive Web-based tailored intervention promoting HPV vaccination acceptability. The 6-step Intervention Mapping (IM) protocol was used to describe the design rationale. After the needs assessment in Step 1, intervention objectives were formulated in Step 2. In Step 3, we translated theoretical methods into practical applications, which were integrated into a coherent intervention in Step 4. In Step 5, we anticipated future implementation and adoption, and finally, an evaluation plan was generated in Step 6. Walking through the various steps of IM resulted in a detailed description of the intervention. The needs assessment indicated HPV vaccination uptake remaining lower than expected. Mothers play the most important role in decision-making about their daughter's immunization. However, they generally feel ambivalent after they made their decisions, and their decisions are based on rather unstable grounds. Therefore, intervention objectives were to improve HPV vaccination uptake and informed decision-making, and to decrease decisional conflict among mothers of invited girls. Computer-tailoring was chosen as the main method; virtual assistants were chosen as a practical application to deliver interactive tailored feedback. To maximize compatibility with the needs of the target group, a user-centered design strategy by means of focus groups and online experiments was applied. In these, prototypes were tested and sequentially refined. Finally, efficacy, effectiveness, and acceptability of the intervention were tested in a randomized controlled trial. Results showed a significant positive effect of the intervention on informed decision-making, decisional conflict, and nearly all determinants of HPV vaccination uptake $(p<.001)$. Mothers evaluated the intervention as highly positive. Using IM led to an innovative effective intervention for promoting HPV vaccination acceptability. The intervention maps will aid in interpreting the results of our evaluation studies. Moreover, it will ease the comparison of design rationales across interventions, and may provide leads for the development of other eHealth interventions. This paper adds to the plea for systematic reporting of design rationales constituting the process of developing interventions. 


\section{BACKGROUND}

Too often design rationales of behavioral intervention programs are poorly described, leading to so-called "black box" evaluations (Kok \& Mesters, 2011). Currently, there is call in the eHealth field to open these black boxes. The scientific literature still provides detailed descriptions of how interventions are evaluated, but hardly ever of what exactly is being evaluated (Schaalma \& Kok, 2009). Moreover, information about when and how decisions are made throughout the process of intervention development is often incomplete or even completely lacking (Dombrovski et al., 2007; Schaalma \& Kok, 2009). Intervention development is a complex and laborious process which requires a large scale of decisions to be made along the way. This goes far beyond the decision about which behavior change techniques to include in an intervention (Dombrovski et al., 2007; Schaalma \& Kok, 2009). We consider all of the decisions to represent valuable knowledge for the scientific community and for intervention developers who like detailed background about the conditions for (in)effectiveness of an intervention. Consequently, all decisions that were made during intervention development should be reported.

Hence, this article aims to provide a detailed, systematic description of the design rationale behind an interactive Web-based tailored intervention promoting HPV vaccination acceptability. This paper encompasses all decisions that were made during the process of intervention development. A systematically developed and well described intervention enables the identification of active ingredients, improvement of existing interventions, future intervention development, and large-scale dissemination (Bartholomew \& Mullen, 2011; Kok \& Mesters, 2011). In addition, it facilitates comparison between interventions, for example for reviews and replication of studies (Gardner et al., 2010; Riley et al., 2011; Web et al., 2010). After all, the usefulness of systematic reviews depends on the quality of the studies included (Dombrovski et al., 2007). Finally, it contributes to theory development by providing insight into causal mechanisms (Bartholomew \& Mullen, 2011; Dombrovski et al., 2007; Michie \& Abraham, 2008; Peters, de Bruin, \& Crutzen, 2013; Webb et al., 2010). We used the Intervention Mapping (IM) protocol, which provides a highly structured approach in describing an intervention program and its development (Bartholomew Eldridge et al., 2016).

\section{METHODS}

IM is a systematic process for developing theory- and evidence-based health promotion interventions. The IM protocol describes the pathways from problem identification to solution (Bartholomew Eldridge et al., 2016). The six steps of IM comprises several tasks, 
each of which integrates theory and evidence. The deliverable of completing the tasks within a step serves as a guide for the subsequent steps. Although IM is presented as a series of steps, Bartholomew Eldredge and colleagues (2016) emphasize that the planning process is iterative instead of linear, meaning that intervention planners move back and forth between the various tasks and steps. By explicitly reporting all decisions and considerations throughout the intervention process, IM makes the intervention development process transparent.

\begin{tabular}{|c|c|c|}
\hline \multirow{6}{*}{ 总 } & $\begin{array}{l}\text { Step 1: Logic Model of } \\
\text { the Problem }\end{array}$ & $\begin{array}{l}\text { - Establish and work with a planning group } \\
\text { - Conduct a needs assesment to create a logic model of the problem } \\
\text { - Describe the context for the intervention including the population, setting and community } \\
\text { - State program goals }\end{array}$ \\
\hline & $\begin{array}{l}\text { Step 2: Program } \\
\text { Outcomes and } \\
\text { Objectives; Logic } \\
\text { Model of Change }\end{array}$ & $\begin{array}{l}\text { - State expected outcomes for behavior and environment } \\
\text { - Specify performance objectives for behavioral and environmental outcomes } \\
\text { - Select determinants for behavioral and environmental outcomes } \\
\text { - Construct matrices of change objectives } \\
\text { - Create a logic model of change }\end{array}$ \\
\hline & $\begin{array}{l}\text { Step 3: Program } \\
\text { design }\end{array}$ & $\begin{array}{l}\text { - Generate program themes, components, scope and sequence } \\
\text { - Choose theory- and evidence-based change methods } \\
\text { - Select or design practical applications to deliver change methods }\end{array}$ \\
\hline & $\begin{array}{l}\text { Step 4: Program } \\
\text { Production }\end{array}$ & $\begin{array}{l}\text { - Refine program structure and organization } \\
\text { - Prepare plans for program materials } \\
\text { - Draft messages, materials, and protocols } \\
\text { - Pretest, refine, and produce materials }\end{array}$ \\
\hline & $\begin{array}{l}\text { Step 5: Program } \\
\text { Implementation Plan }\end{array}$ & $\begin{array}{l}\text { - Identify potential program users (implementers, adopters, and maintainers) } \\
\text { - State outcomes and performance objectives for program use } \\
\text { - Construct matrices of change objectives for program use } \\
\text { - Design implementation interventions }\end{array}$ \\
\hline & $\begin{array}{l}\text { Step 6: Evaluation } \\
\text { Plan }\end{array}$ & $\begin{array}{l}\text { - Write effect and process evaluation questions } \\
\text { - Develop indicators and measures for assessment } \\
\text { - Specify the evaluation design } \\
\text { - Complete the evaluation plan }\end{array}$ \\
\hline
\end{tabular}

Figure 4.1 Intervention Mapping steps and tasks (adapted from Bartholomew Eldrigde and colleagues, 2016).

Step 1 concerns the conduction of a needs assessment and formulation of the overall goals of the intervention. In this step, the health problem, behavioral and environmental causes of this problem, and related determinants are identified. The intervention goal is the desired outcome of the intervention. In Step 2, performance objectives and change objectives are formulated. Performance objectives (POs) specify the (sub)behaviors that must be performed by the target group in order to reach the intended goal. Change objectives (COs) outline the specifics of behavioral determinants to be targeted so the target group is enabled to reach the performance objectives. COs are formed by crossing the POs with the determinants. This results in a matrix which can be seen as the core of 
the design rationale. Step 3 is about the design of the intervention program in terms of generating program themes, components, scope and sequence. The scope is the breadth and amount of the program and the sequence is the order in which programs are delivered across time. This step also includes the selection of theory-based intervention methods and the translation of these methods into practical applications, taking into account the parameters for effectiveness of the these methods. In Step 4, the methods and practical applications are being translated into a coherent intervention program. In Step 5, adoption, implementation, and sustainability of the intervention in real-life settings are planned. Finally, Step 6 entails the outline of the process and effect evaluation. The steps and tasks of IM are visualized in Figure 4.1.

\section{RESULTS}

Systematically walking through all of the steps of IM, resulted in a detailed description of the intervention. This description, in turn, provides insight into the theory- and researchbased foundations of the many decisions that were made during the process of intervention development. Below, the study findings from each step of IM are described.

\section{IM Step 1: Needs assessment}

Worldwide, cervical cancer is the third most common cancer among women (Ferlay et al., 2010). Persistent infection by the human papillomavirus (HPV) appears to be the major cause of cervical cancer (Schiffman \& Castle, 2003). In the Netherlands, yearly 600 new cases of cervical cancer are detected, of which 200 with fatal consequences (Gezondheidsraad, 2008). This is despite the presence of a national cervical cancer screening program for women aged 30-60 years (Braspenning et al., 2001). HPV vaccinations of 12-year-old girls were expected to reduce the number of cervical cancer cases by $50 \%$ (Gezondheidsraad, 2008). Therefore, in 2008, the Dutch government decided to include the HPV vaccination of 12 -year-old girls in the National Immunization Program (NIP). However, HPV-vaccine uptake remains lower (61\%) than expected (70\%) (Van Lier et al., 2016). There is a need to improve this uptake is order to reduce the cervical cancer burden. Therefore, the primary goal of the intervention was to improve HPV vaccination uptake among invited girls.

Most studies have confirmed that parents play a large role in decision-making about their daughters' HPV vaccination (e.g., Brewer \& Fazekas, 2007; Dahlstrom et al., 2010; Gerend, Weibly, \& Bland, 2009). A Dutch study showed mothers to play the most important role in the immunization decision of girls. There is a high percentage of consensus between mothers and daughters (79\%) about the outcome (Van Keulen et al., 2010). Therefore, mothers were selected as the target group for designing an educational intervention for 
promoting the HPV vaccination uptake by their daughters. In order to gain insight into determinants of mothers' HPV vaccination acceptability, we conducted a longitudinal study (Pot et al., 2017b). The results showed that intention was the main and stable predictor of HPV vaccination uptake. Intention, in turn, was best explained by attitude, be Van Keulen et al., 2013a; Van Keulen et al., 2010).

Furthermore, research showed that a substantial proportion of the mothers had not actively processed information about the HPV vaccination (50\%) and still felt ambivalent after they made their decision (25\%) (Van Keulen et al., 2010). This indicates that the mothers' decision is based on rather unstable grounds, which makes them vulnerable for arguments challenging their initial attitudes and/or intention. Informed decision-making is expected to make mothers less vulnerable for counter arguments (McGuire, 1964; Paulussen et al., 2006). Furthermore, decisional conflict is strongly related to informed decision-making, as one of the factors contributing to decisional conflict is feeling uninformed (O'Connor, Jacobsen, \& Stacey, 2002). A more informed decision is thus theoretically related to reduced decisional conflict (Biesecker, Schwartz, \& Marteau, 2013). Hence, the secondary goal of the intervention is to improve informed decision-making, reduce decisional conflict, and positively influence determinants of HPV vaccination uptake.

In the Netherlands, the existing education about the HPV vaccination consists of an introduction folder and a link to a website providing generic information. All girls at the age of 12 receive an invitation for the HPV vaccination with the accompanying brochure and link to the website. But, this education needs to be improved because HPV vaccination uptake remains lower than expected (Van Lier et al., 2016). Besides, Van Keulen and colleagues (2010) showed that mothers indicated a need for more interactive, personal information about the HPV vaccination over and above the general information. Mothers also expressed differential needs concerning the amount and scope of information. Topics of interest for future communication about the HPV vaccination as indicated by mothers were for example, the pros and cons, potential long-term side effects, and the safety and effectiveness of the HPV vaccination (Van Keulen et al., 2010). Regarding the delivery mode of the information, mothers indicated a preference for internet (Van Keulen et al., 2010). These preferences guided our decision to develop a Web-based intervention, enabling us to provide mothers with interactive, tailored information about the HPV vaccination (see Step 3). 


\section{IM Step 2: Program Outcomes and Objectives}

\section{Program Outcomes}

Based on the identified problem and needs we determined that the primary outcome was to improve HPV vaccination uptake among invited girls and the secondary outcome was to strengthen mothers' informed decision-making, reduce decisional conflict, and positively influence determinants of the HPV vaccination decision. Below, we introduce POs, determinants of these PO's, and accompanying COs for each outcome.

\section{Performance Objectives}

The expected intervention outcomes were subdivided into POs. The HPV vaccination consists of two subsequent injections. In other words, the behavior has to be repeated only once (with an interval of 6 months). The POs are: (1) the mother makes a (informed) decision to have her daughter vaccinated against HPV; (2) the mother discusses her decision with her daughter and partner; (3) the mother guides her daughter towards receiving the first HPV injection; (4) the mother guides her daughter towards receiving the second HPV injection.

\section{Behavioral Determinants}

For each PO, we identified the reasons why mothers would take that action. These so called behavioral determinants were based on theory (e.g., the theory of reasoned action and socio-cognitive theory) (Fishbein \& Ajzen, 2010; Bandura, 1986, respectively) and on empirical research (Van Keulen et al., 2013a). We selected determinants that met the criteria of importance and changeability (Bartholomew Eldridge et al., 2016). Importance of the determinants of PO 1 (i.e., deciding to get the HPV vaccination) was based on the association (i.e., R2 effect size) (Cohen, 1988) of the determinants with HPV vaccination intention (Van Keulen et al., 2013a). For the POs 2 to 4 (i.e., discussing the HPV vaccination and actually getting the first and second injection), importance was based on consensus among co-authors (RR, TP, MP and HVK). Changeability (i.e., the strength of the evidence that the proposed change can be realized by the intervention), was also based on consensus among co-authors (RR, TP, MP and HVK).

For the first PO, we returned to the needs assessment and selected the following determinants: attitude, beliefs, positive and negative outcome expectancies, anticipated regret about both receiving and rejecting the HPV vaccination, confidence in authorities, habit strength, risk perception having received (no) HPV vaccination, subjective and descriptive norms, relative effectiveness of the HPV vaccination, ambivalence, and HPV vaccination information processing (Van Keulen et al., 2013a; Van Keulen et al., 2010). Furthermore, according to Marteau and colleagues (2001), an informed decision is based 
on sufficient and relevant knowledge, and a match between the person's values (i.e., their attitude towards the HPV vaccination) and outcome behavior (i.e., whether mothers had their daughter vaccinated against HPV or not). Consequently, knowledge was selected as a determinant. Determinants that were selected for PO2 were attitude (Fishbein \& Ajzen, 2010), and self-efficacy (Bandura, 1986). Selected determinants for PO3 and PO4 were knowledge and beliefs (Bandura, 1986). Determinants that were not selected were considered either unimportant (e.g., for PO1: self-efficacy and past cancer experience) (Van Keulen et al., 2010; Van Keulen et al., 2013a) or unchangeable (e.g., for PO2: parenting style).

\section{Change Objectives}

For each determinant, we identified change objectives COs. COs are actions required in order to achieve the performance objectives, and hence the outcomes. They are the active ingredients of the intervention and function as a blueprint of the theoretical design rationale. Table 4.1 provides an overview of examples of the matrix of COs, the complete version can be found in Appendix B.

Table 4.1 Examples of change objectives (COs).

\begin{tabular}{|c|c|c|c|c|}
\hline \multirow[b]{2}{*}{$\begin{array}{c}\text { Performance } \\
\text { Objective }\end{array}$} & \multicolumn{4}{|c|}{ Determinant } \\
\hline & Knowledge & Attitude & Beliefs & $\begin{array}{c}\text { Risk perception } \\
\text { having received no } \\
\text { HPV vaccination }\end{array}$ \\
\hline $\begin{array}{l}\text { 1. Mother makes } \\
\text { the (informed) } \\
\text { decision to have her } \\
\text { daughter vaccinated } \\
\text { against HPV. }\end{array}$ & $\begin{array}{l}\text { Mother explains that } \\
\mathrm{HPV} \text { is a virus. } \\
\text { Mother explains that } \\
\mathrm{HPV} \text { is transmitted } \\
\text { sexually. } \\
\text { Mother explains } \\
\text { that men can also be } \\
\text { infected with HPV. }\end{array}$ & $\begin{array}{l}\text { Mother evaluates } \\
\text { the HPV } \\
\text { vaccination } \\
\text { positively. } \\
\text { Mother recognizes } \\
\text { the health benefits } \\
\text { of the HPV } \\
\text { vaccination. }\end{array}$ & $\begin{array}{l}\text { Mother recognizes } \\
\text { the importance of her } \\
\text { daughter receiving the } \\
\text { HPV vaccination before } \\
\text { they become sexually } \\
\text { active (i.e., age 12). } \\
\text { Mother recognizes that } \\
\text { the vaccine has proven } \\
\text { to be safe and effective. }\end{array}$ & $\begin{array}{l}\text { Mother acknowledges } \\
\text { the risk of her } \\
\text { daughter becoming } \\
\text { infected with HPV } \\
\text { and developing } \\
\text { cervical cancer } \\
\text { later in life without } \\
\text { the vaccination. }\end{array}$ \\
\hline $\begin{array}{l}\text { 2. Mother discusses her } \\
\text { decision to have her } \\
\text { daughter vaccinated } \\
\text { against HPV with her } \\
\text { daughter and partner. }\end{array}$ & & $\begin{array}{l}\text { Mother evaluates } \\
\text { communication } \\
\text { with her daughter } \\
\text { and partner } \\
\text { positively. }\end{array}$ & & \\
\hline $\begin{array}{l}\text { 3. Mother guides her } \\
\text { daughter towards } \\
\text { receiving the first } \\
\text { HPV injection. }\end{array}$ & $\begin{array}{l}\text { Mother knows } \\
\text { where to get the first } \\
\text { HPV injection. }\end{array}$ & & & \\
\hline $\begin{array}{l}\text { 4. Mother guides her } \\
\text { daughter towards } \\
\text { receiving the second } \\
\text { HPV injection. }\end{array}$ & $\begin{array}{l}\text { Mother knows where } \\
\text { to get the second } \\
\text { HPV injection. }\end{array}$ & & $\begin{array}{l}\text { Mother recognizes that } \\
\text { the HPV vaccination } \\
\text { is most effective when } \\
\text { her daughter gets } \\
\text { fully vaccinated. }\end{array}$ & \\
\hline
\end{tabular}




\section{IM Step 3: Program Design}

\section{Theme, components, scope and sequence}

The first task of Step 3 is to generate ideas for intervention theme, components, scope, and sequence. The product of this step is an initial plan that describes the program (Bartholomew Eldrigde et al., 2016). We decided the intervention to be Web-based (see Step 1). The main theme of the intervention was 'making an informed decision about the HPV vaccination of your daughter'. The various components were logically clustered for improving usability. This resulted in four menu options: (1) two-sided information about the HPV vaccination, (2) a decisional balance, (3) practical information, and (4) frequently asked questions (See Appendix C for screenshots of the four menus). The first menu enabled mothers to collect tailored information about the HPV vaccination (e.g., such as information about the effectiveness of the HPV vaccination). The various components were in line with mothers' preferences as indicated by earlier research (See 'needs assessment'). In the second menu, mothers could weigh their personal values regarding the HPV vaccination in the form of a decisional balance and values clarification tool. In the third menu, mothers could gather practical information such as how and where to receive the HPV vaccination. The fourth menu listed frequently asked questions about the HPV vaccination. Here we also added a 'problems with the website' component, providing mothers with help.

Furthermore, mothers were able to visit the intervention multiple times. The first time they visited the website, they were provided with an explanation of how the website worked. Then, they were introduced to the first menu. We used a combination of a freedom of choice and a tunneled design (i.e., a "hybrid design") to guide mothers through the website (Danaher, McKay, \& Seeley, 2005). This means that mothers could choose themselves which component in which menu they wanted to visit (i.e., freedom of choice design). However, once they entered a component, they were guided through it in "tunnel fashion", with navigation being limited to "next" and "prior" buttons. The reason for choosing such a hybrid design is that we wanted to profit from the strengths of both approaches (Danaher, McKay, \& Seeley, 2005). Specifically, the tunnel design was expected to increase intervention adherence and engagement and acquisition of knowledge (Crutzen, Cyr, \& De Vries, 2012). The freedom of choice design was expected to promote autonomy of the mother, which is important when motivating behavior change (Miller \& Rollnick, 2013; Ryan et al., 2011). Furthermore, the hybrid design matched the differential needs concerning the amount and scope of information expressed by the mothers (Van Keulen et al., 2010). Table 4.2 provides an overview of the scope and sequence of the intervention. 
Table 4.2 Scope (components and main targeted determinants) and sequence of the intervention.

\begin{tabular}{|c|c|}
\hline Menu $^{\mathrm{a}}$ & Component ${ }^{\mathrm{b}}$ : main targeted determinants \\
\hline $\begin{array}{l}\text { Information About the } \\
\text { HPV vaccination }\end{array}$ & $\begin{array}{l}\text { - General information: knowledge } \\
\text { - Facts and stories: beliefs, positive and negative outcome expectancies } \\
\text { - From HPV to cervical cancer: knowledge } \\
\text { - Ways to protect against cervical cancer: relative effectiveness } \\
\text { - Side effects of the HPV vaccination: negative outcome expectancies } \\
\text { - Importance vaccinating at young age: positive outcome expectancies } \\
\text { - Other mothers: descriptive norm } \\
\text { - Working mechanisms vaccination: knowledge } \\
\text { - Chance of getting HPV/cervical cancer: risk perception having received (no) HPV vaccination } \\
\text { - Effectiveness and safety of the HPV vaccination: beliefs, positive outcome expectancies }\end{array}$ \\
\hline $\begin{array}{l}\text { Weighing up the } \\
\text { pros and cons }\end{array}$ & $\begin{array}{l}\text { - Decisional Balance: attitude, ambivalence } \\
\text { - Values clarification: attitude, ambivalence }\end{array}$ \\
\hline Practical information & $\begin{array}{l}\text { - Talking about the HPV vaccination: attitude, self-efficacy, subjective norms } \\
\text { - Where do I get the HPV vaccination: knowledge, planning, self-efficacy } \\
\text { - } 2 \text { instead of } 3 \text { HPV injections: knowledge }\end{array}$ \\
\hline $\begin{array}{l}\text { Frequently asked } \\
\text { questions }\end{array}$ & $\begin{array}{l}\text { - Frequently asked questions about the HPV vaccination: n/a } \\
\text { - Frequently asked questions about getting the HPV vaccination: } \mathrm{n} / \mathrm{a} \\
\text { - Problems with the website: } \mathrm{n} / \mathrm{a}\end{array}$ \\
\hline
\end{tabular}

Note: $\mathrm{n} / \mathrm{a}=$ not applicable ${ }^{\mathrm{a}}$ 'Within and across the different menus, a freedom of choice design was used; ${ }^{\mathrm{b}}$ Within the various components, a tunnel design was used.

\section{Theoretical Methods and Practical Applications}

To identify theoretical change methods that help achieve the COs, we used an overview of methods provided by Bartholomew and colleagues (chapter 6) (Bartholomew Eldridge et al., 2016). The eHealth setting gave us the chance to apply effective strategies in an innovative way, namely by using computer-tailoring and interactions with virtual assistants (See sections below) (Noar \& Harrington, 2012). Computer-tailoring was selected as the main theoretical framework for development. Tailoring is a health communication strategy by which messages are individualized to personal preferences and needs (Kreuter \& Wray, 2003). Meta-analyses have shown that tailored interventions are more effective than generic interventions in achieving behavioral outcomes (e.g., Krebs, Prochaska, \& Rossi, 2010; Lustria et al., 2013). Beneficial effects of tailoring are attributed to improved exposure, information processing, appreciation, reading, and perceived personal relevance (e.g., Brug, Oenema, \& Campbell, 2003; Ruiter et al., 2006). Because computer-tailored interventions can reach large groups of people at relatively low costs, especially when delivered via the Internet (Neville, O'Hara, \& Milat, 2009), they can have substantial impact at the population level (Noar, Benac, \& Harris, 2007). Also, tailoring matches the mothers' need for more interactive, personal information about the HPV vaccination (Step 1) (Van Keulen et al., 2010). 
Computer-tailored feedback was used in three different ways throughout the intervention. First, it was used to tailor the feedback on participants' answers to statements and questions about specific aspects of the HPV vaccination. For instance, mothers were first asked to estimate their daughters' chance to get an HPV-infection. Those who perceived this chance as low, received feedback which stated that this chance is rather high instead of low, whereas those who perceived the chance as high, received feedback that confirmed that the chance is indeed high. Second, computer tailoring was used to provide mothers the opportunity to weigh their personal values regarding the HPV vaccination in a decisional balance. Another mean was the 'value clarification' tool (a motivational interviewing strategy; cf. Miller \& Rollnick, 2013). Mothers were invited to list their central values for life, and were stimulated to relate these to the decision about vaccinating her daughter. Finally, computer-tailoring was used for guiding mothers through the website. The intervention kept track of the components that the mother had already visited by using logs. This enabled us, for instance, to highlight parts of the intervention which the mother had not seen yet. Also, if mothers were exposed to information that had already been discussed in another component, the intervention mentioned this in order to assure that the connection between the different types of information provided was clear.

We selected virtual assistants for delivering tailored feedback. A virtual assistant is an embodied conversational agent defined as a computer program with a human-like visual make-up and appearance on a computer screen (Van Vugt, 2008). Virtual assistants were chosen to match the mothers' preferences for more interactive personalized feedback (Van Keulen et al., 2010). They provide opportunities for two-way interactions, and can create a highly personal experience. Also, research has indicated that a social relationship between user and program is important (Bickmore \& Picard, 2005; Okun, 2008), as it supports the basic psychological need for relatedness (Ryan et al., 2011). This can be established by using virtual assistants (Baylor, 2009; Baylor, 2011; Mennecke et al., 2011). Also, several studies confirmed that the presence of a virtual assistant can further improve the effectiveness of the intervention (Blanson Henkemans et al., 2009; Jin, 2010; Watson et al., 2012). Specifically, the added value of using a virtual assistant over a text and picture-based website is that it improves recall of information (Beun, de Vos, \& Witteman, 2003), transfer of learning (Atikinson, 2002), amount of learning (Baylor \& Kim, 2004), self-efficacy expectations, literacy and behavior change (Blanson Henkemans et al., 2009; Blanson Henkemans et al., 2008; Jin, 2010). In addition, the mere presence of such an animated interface agent has a positive effect on experiencing fun and engagement (e.g., Baylor, 2009; Jin, 2010; Wik \& Hjalmarsson, 2009). Two virtual assistants were visualized: a mother-like and a female doctor-like assistant as the combination of using an expert and a peer virtual assistant has been shown to be effective (Durantini et al., 2006; Hopfer, 2012). The main purpose of the virtual assistants was to provide mothers with social support, which is an important factor 
associated with positive health outcomes in general (Bickmore et al., 2010). The motherlike assistant was used to guide mothers throughout the website and helped weigh their personal values in the decisional balance. The doctor-like assistant was used to deliver feedback about the HPV vaccination.

Table 4.3 provides examples of theoretical methods (column 2) for determinants identified in IM step 2 (column 1) for PO1 (i.e., 'mother makes the (informed) decision to have her daughter vaccinated against HPV). For each method, parameters for effectiveness were specified (column 3). We then translated theoretical methods into practical applications (column 4) that were appropriate for the population and the (Internet) setting. In Appendix $\mathrm{D}$, we also specify which POs and COs were targeted using which methods and applications in the various components. This can be seen as the most straightforward blueprint of the intervention. All COs were covered by the intervention.

The most important method aiming to reduce decisional conflict was the decisional balance (see Figure 4.2), which has proved a quick and efficient intervention by itself (Labrie et al., 2006). Mothers were presented with a list of pros and cons of the HPV vaccination by the mother-like assistant (left column). This list was based on pros (e.g., 'the HPV vaccination decreases the chance of my daughter getting cervical cancer') and cons (e.g., 'my daughter is too young to receive the HPV vaccination') that were considered most important to the mothers as indicated by the needs assessment (Step 1). For each pro or con, they indicated (1) whether they agreed (disagree / neutral / agree; middle column) and (2) how important the pro or con was to them (unimportant / neutral / important; third column). The latter was indicated by stars: the more stars, the more important the pro or con was to the mother. When mothers (dis)agreed, tailored feedback 'popped up. This was done to ensure mothers based their answer on correct information (see Figure 4.2). Furthermore, mothers were given the option to add pros and cons that were not in the list. Based on pros and cons mothers marked as most salient, a decisional balance (top right of the screen) revealed their current position on a scale ranging between not-wanting (left side) and wanting (right sight) to get their daughter vaccinated. 


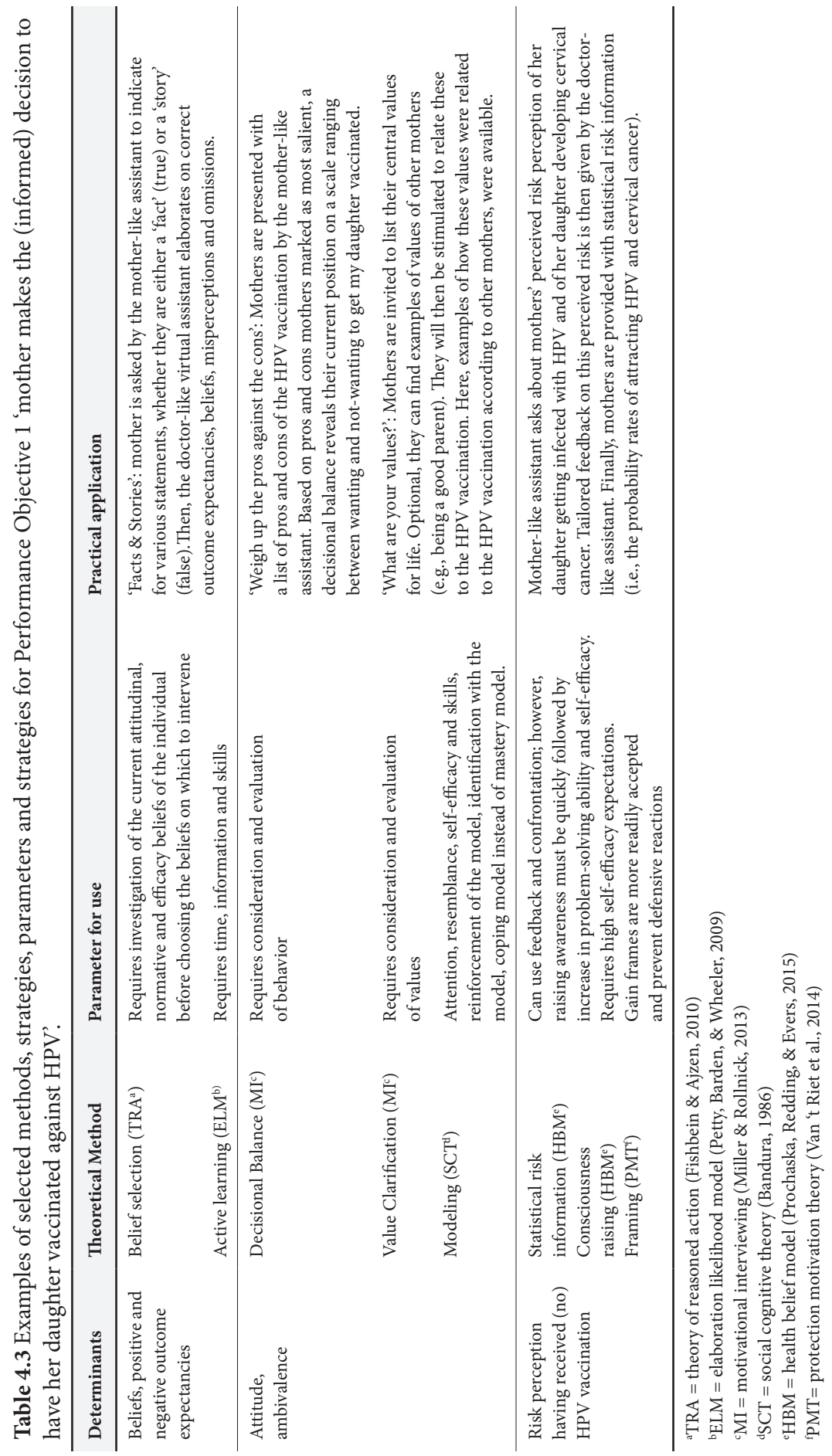




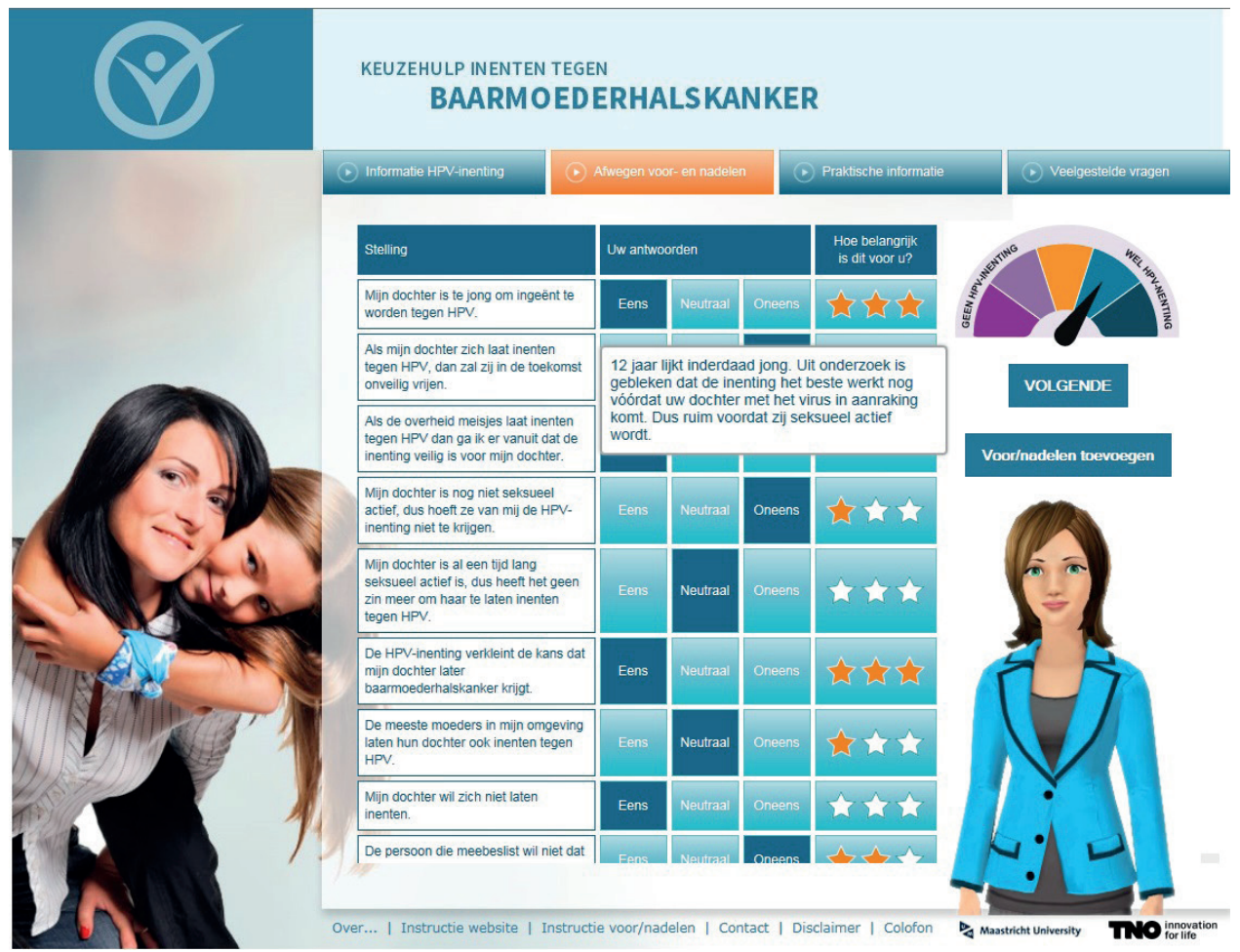

Figure 4.2 Screenshot of the decisional balance with a tailored pop-up and the mother-like virtual assistant on the website.

Note: The plan for the decisional balance was developed in step 3; actual development of the balance was realized in step 4.

\section{IM Step 4: Program Production}

We developed the intervention using Tailorbuilder (c) software. The virtual assistants were developed by a company called 'Webspeaking'. Individual responses and routing were linked to written and spoken feedback messages by means of computer software using if-then algorithms. The website was made available on computers and tablets and was OS-platform independent. Using an online questionnaire, mothers $(\mathrm{N}=375)$ were asked about the preferred graphical appearance of the intervention (including the name of the intervention, the voices and appearances of the virtual assistants). A text-editor rephrased the written and spoken texts in order to maximize comprehensibility. A graphic designer made the website design and provided us with appropriate pictures to illustrate feedback, in order to make the website more appealing for this target group (See Figure 4.3 for an example). 


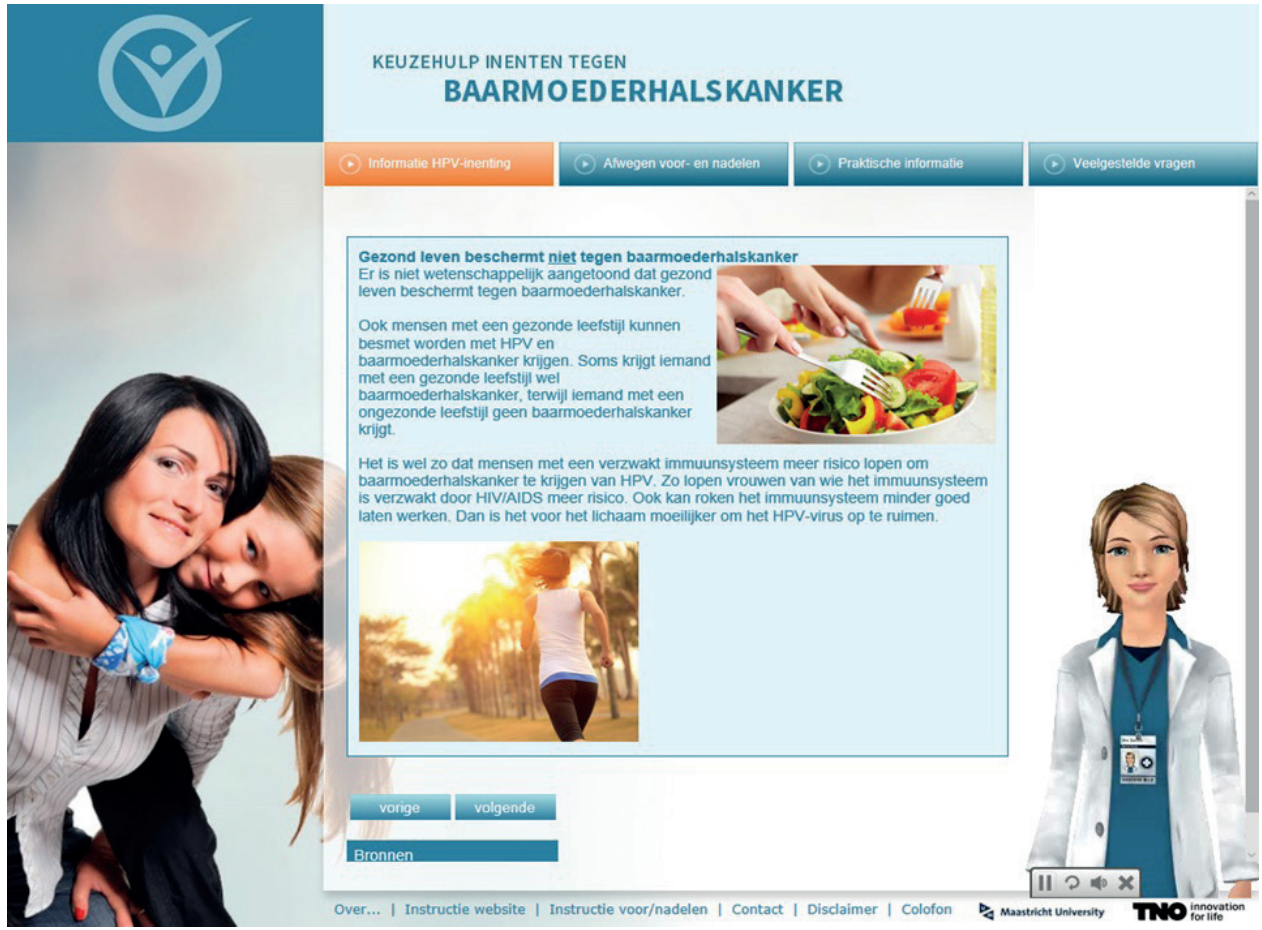

Figure 4.3 Screenshot of the doctor-like assistant providing feedback about the ineffectiveness of having a healthy life style (e.g., healthy eating, exercising) in protecting against cervical cancer with illustrations provided by a graphic designer.

\section{Experimental pre-testing and pilot-testing of prototypes}

In order to anticipate an intervention that meets the requirements and preferences of the target group, we followed user-centered design procedures. This entails the iterative involvement of the end-users in the design process (Mao et al., 2005). We gathered feedback on different versions (static and interactive) or (parts of the) intervention, by online experimental pretests and focus groups respectively. Experimental pretesting offers empirical support for the impact of strategies on determinants and serves as a guarantee for implementing adequate intervention materials (Whittingham et al., 2008a; Whittingham et al., 2008b). We conducted three experimental online pretests. Since we did not find a clear consensus in the literature about the framing of risks, the first experiment $(\mathrm{N}=375)$ was about testing the differential effect of providing statistical (i.e., probability rates) or/ and narrative risk information (i.e., a personal story). Mothers were randomly assigned to one of four conditions in a 2 (statistical information: yes or no) x 2 (narrative information: yes or no) between-subjects factorial design. ANOVA revealed a significant main effect 
of statistical information on perceived daughters' susceptibility towards $\operatorname{HPV}(F(1,371)$ $=7.56, p<.01)$. Mothers who received statistical risk information had a higher perceived daughters' susceptibility towards HPV $(M=4.11$ on a 7-point scale, $S D=.10)$ than mothers who did not receive statistical risk information $(M=3.73$ on a 7 -point scale, $S D=.09)$. Thus statistical risk information seemed most effective. We therefore decided to include statistical risk information to target risk perception with this intervention (Step 3).

In a second online experimental pretest $(\mathrm{N}=561)$, we explored the best way to communicate about social norms; by providing negatively (i.e., discourage undesired behavior) versus positively (i.e., encourage desired behavior) framed descriptive and/or subjective norms (Cialdini et al., 2006). It was suggested that the descriptive norm should be avoided in situations where the unhealthy behavior is prevalent (Mollen, Ruiter, \& Kok, 2010). As for the HPV vaccination, 39\% of invited girls have not received the HPV vaccination (Van Lier et al., 2016). Therefore, we also wanted to examine whether communicating a descriptive norm could have a potential adverse effect on HPV vaccination acceptability. Mothers were randomized into one of four conditions in a 2 (norm: injunctive versus descriptive) x 2 (frame: positive versus negative) between subjects factorial design with an additional control condition. We found no indication for using one type of framing norms over the other ( $p$ 's > .05; mean HPV vaccination intention scores ranging from 5.51 to 5.77, on a 7-point scale). We also did not find any adverse effects of descriptive norms on HPV vaccination acceptability $(p>.05)$. As negatively framed norms were expected to be more difficult to process (Gilbert, 1991), we decided to just include positively framed descriptive norm by communicating about the national HPV vaccination uptake rate. Within the component 'talking about the HPV vaccination', mothers were taught how to deal with a potential contrasting subjective norm of important others (e.g. their daughter and partner).

Finally, being a relatively new vaccine, there remains uncertainty about potential long-term effects of the HPV vaccination. This was also found to be a topic of interest among mothers for future communication (Van Keulen et al., 2010). Therefore, in a third experimental pretest $(\mathrm{N}=695)$, we investigated the effects of acknowledging versus ignoring uncertainty about potential long-term effects of the HPV vaccination. Mothers were randomly assigned to one of two conditions in which uncertainty about the HPV vaccination was either (a) acknowledged or (b) ignored. Results showed that, compared to mothers who were exposed to information ignoring uncertainty, mothers who were exposed to information acknowledging uncertainty experienced more decisional conflict (acknowledged: $M=$ $3.42, S D=1.84, p<.01$ versus ignored: $M=3.05, S D=1.74)$, were more ambivalent about their decision (acknowledged: $M=4.04, S D=1.86$ versus ignored: $M=3.42, S D=1.89, p$ $<.001$ ), and had a less positive attitude (acknowledged: $M=5.07, S D=1.50$ versus ignored: $M=5.69, S D=1.38, p<.01$ ) and intention (acknowledged: $M=5.26, S D=1.73$ versus 
ignored: $M=5.85, S D=1.45, p<.01)$. These findings implicate not to communicate about long-term uncertainties. However, we chose to do so in the intervention, for the following reasons: first, the found effect sizes were small. Second, not communicating about long term uncertainties brings along the risk of mothers searching information about this elsewhere. This can be quite dangerous as many rumors about potential long-term effects, for which no prove exists, can be found (e.g., on the Internet) (Kata, 2012). Reading these (false) rumors without any refutation being offered aside (Allen, 1991), could have more detrimental effects on HPV vaccination acceptability than when we ourselves provide the (correct) information. The latter enables us to inoculate mothers with arguments that become accessible in case they are confronted with (new) information that might challenge their initial positive intentions (i.e., psychological inoculation) (McGuire, 1964; Paulussen et al., 2006). Finally, mothers themselves expressed a need for full disclosure, especially when uncertainties were ignored, which was also found in a previous study (Van Keulen et al., 2010).

At a later stage, we conducted several focus groups $(\mathrm{N}=3)$ among mothers to ensure compatibility with the preferences of the target group. A first prototype of the intervention was tested in two focus groups. After we revised the prototype according to the feedback from these two focus groups, a second prototype of the intervention was tested in a third focus group. The protocol was similar for all focus groups: after a general introduction, mothers were given a laptop and headset to individually navigate through the website. They were given the opportunity to give feedback on every page of the website about features they (dis)liked (e.g., the "look and feel" of the page(s), and the tailored feedback of the virtual assistants). Then, they were asked to fill out a written questionnaire assessing their subjective evaluation of the virtual assistants (e.g., the extent to which feedback matched their responses) and the website (e.g., their evaluation of the different menus). Finally, in a group discussion mothers could elaborate on their opinion about the intervention, and offer suggestions for improvement.

Feedback was first gathered from the first two focus groups. For instance, in the first prototype, there was a component targeting anticipated regret by using imagery (Steen, 2007; Wright, 2011). Mothers were asked how much regret they would have if they did not vaccinate their daughter against HPV and their daughter developed cervical cancer later in life. However, we discovered that asking this evoked much resistance. We therefore decided to remove this component from the intervention. As an alternative, we decided to target anticipated regret indirectly (e.g., by giving information about the high prevalence of HPV). Furthermore, in the first prototype, the written and spoken tailored feedback were provided 
at the same time. Mothers indicated that, therefore, they experienced difficulties listening to the virtual assistant. Hence, we created a new prototype, in which the written feedback appeared once the virtual assistant was done providing the tailored feedback.

In the third focus group, mothers indicated that they would like to see which components they had already visited. We therefore created an adapted version of the website in which logs were used to register the pages mothers had already visited and subsequently used these logs to visualize which components were completed. This was done by turning them into a different color (i.e., orange, See Figure 4.4). In addition, the mother-like virtual assistant was used to give advice about components to visit next, in order to maximize exposure to the intervention. If the virtual assistant advised on a component, the component was highlighted by an orange circle (See Figure 4.4).

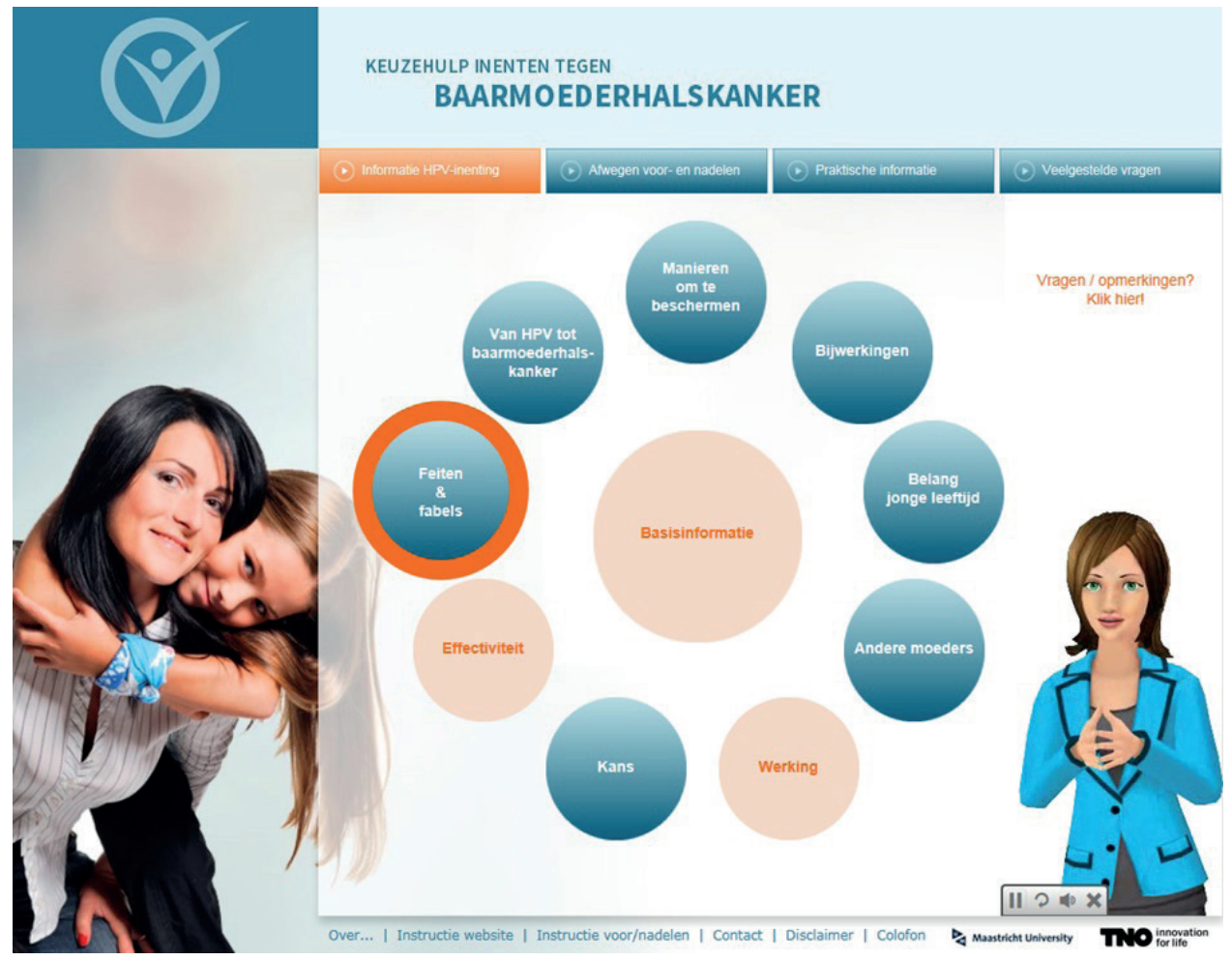

Figure 4.4 Screenshot of the first menu with the mother-like assistant in which a suggested component is highlighted and visited components have turned into a different color. 
After revising the intervention according to the feedback from the third focus group, a final prototype was pilot-tested online using various devices to ensure it worked adequately. This was done among a sample of mothers $(\mathrm{N}=10)$ and among members of the project group.

\section{IM Step 5: Designing an implementation plan}

To ensure future implementation and adoption of the intervention (step 5), we formed an advisory board of representatives of important linking agents (e.g., Public Health Services) and professionals involved in delivering the HPV vaccination. We organized two advisory board meetings; they advised on the experimental pretesting, practicability and feasibility of the intervention, the planned effect- and process evaluation, and implementation of the intervention within the NIP. The National Institute for Public Health and the Environment (RIVM), responsible for the national implementation of HPV vaccination, was co-financier of the project and full member of the project team. RIVM would get full control and management over the website if the final intervention turned out to be effective.

\section{IM Step 6: Creating an evaluation plan}

In order to evaluate the efficacy and effectiveness of the intervention, we planned a randomized controlled trial (RCT). The RCT consisted of 2 arms: (1) a control and (2) intervention group. Mothers were randomly recruited from Praeventis, the Dutch National Immunization Register, and three Internet panels. The latter was to guarantee a suitable subsample for the planned efficacy trial (Van Keulen et al., 2013a). The Praeventis sample enabled us to anticipate the naturalistic condition for future implementation of the intervention, which provided the opportunity for testing the intervention's effectiveness. The primary outcome measure was HPV vaccination uptake, as registered by Praeventis Secondary measures were informed decision-making, decisional conflict, and determinants of HPV vaccination acceptability. These were measured using a Web-based questionnaire.

Part of the RCT was a process evaluation assessed program adherence and the users' subjective program evaluation. At follow up, participants evaluated the information provided by the website (e.g., relevance, credibility), perceived user control (e.g., experienced degree of autonomy) and the functioning of the virtual assistants (e.g., fun, reliability). Mothers were also asked to rate the website and the virtual assistants on a 10-point scale, ranging from 0 (very bad) to 10 (excellent). Objective program use was evaluated by the logs keeping track of the pages the mothers' has visited. Two indicators were computed: "completeness" and "time". Completeness represents the total percentage of pages that a participant has visited while logged into the website, ranging from 0\% (no exposure) to $100 \%$ (exposure to all pages). Time represents the total amount of time participants have spent logged into the intervention. 
Results from the RCT are described in detail elsewhere (Pot et al., 2017a). The main finding from the effect evaluation was that the intervention showed a significant positive effect on informed decision-making, decisional conflict, and nearly all determinants of HPV vaccination uptake $(p<.001)$. No differences in intervention effects were found between the two differential samples. The main finding from the process evaluation was that mothers evaluated the intervention as highly positive: mothers evaluated the website with a 7.6 (SD $=1.36)$ and the virtual assistants with a $7.4(S D=1.53)$. According to the computer logs, $2,509(63 \%)$ of the $3,995(100 \%)$ invited mothers logged on to the website. On average, mothers spent 22 minutes on the website ( $S D=13$ minutes).

\section{DISCUSSION}

In this article, we have provided a comprehensive and detailed description of how we systematically developed an intervention promoting HPV vaccination acceptability using the IM protocol. This led to a highly innovative, interactive, Web-based, tailored intervention, in which tailored feedback was delivered by virtual assistants. Tailoring has only recently been applied to HPV vaccination (Bennett et al., 2013; Gerend et al., 2013; Grandahl et al., 2016; Hopfer, 2012). To our knowledge, only one of the existing tailored interventions was computer-tailored and this intervention turned out to be ineffective in promoting HPV vaccination acceptance (Bennett et al., 2013). Moreover, not only did we tailor the content of the intervention to the mothers' personal interest, but tailoring was also used to guide the mothers' personal pathway through the intervention. The latter is likely to have improved the usability of the intervention. The intervention accounted for tailoring on a variety of determinants. For example, not only did we tailor on perceived barriers (e.g., beliefs about adverse effects), like Gerend and colleagues (2013) did, but also on other beliefs (e.g., beliefs about the daughters' sexual behavior and age in relation to the need for the HPV vaccination), attitude, subjective norms, habit, relative effectiveness, anticipated regret, risk perception, self-efficacy and knowledge. The use of virtual assistants in interventions promoting HPV vaccination acceptance seems promising since results from the focus groups (Step 4) and the subjective program evaluation (Pot et al., 2017a) showed that mothers appreciated them very well. But, we still consider the use of virtual assistants to be complex, especially in Web-based interventions in which both spoken and written feedback/information are provided.

The intervention appeared effective in promoting HPV vaccination acceptability and informed decision-making, and appeared to have potential for broad scale dissemination and implementation (Pot et al., 2017a). This intervention blueprint will aid in interpreting the results of our evaluation studies (Pot et al., 2017a). In addition, it provides insight into 
causal mechanisms, which contributes to theory development (Bartholomew \& Mullen, 2011; Dombrovski et al., 2007; Michie \& Abraham, 2008; Peters, de Bruin, \& Crutzen, 2013; Webb et al., 2010). Moreover, it will ease the comparison of design rationales across interventions (e.g., for reviews and replication of studies) (Gardner et al., 2010; Riley et al., 2011; Web et al., 2010). Finally, it provides leads for the development of other eHealth interventions (Bartholomew \& Mullen, 2011; Kok \& Mesters, 2011).

\section{Advantages of IM}

We believe that using IM greatly contributed to the intervention being effective in promoting HPV vaccination acceptability and informed decision-making among mothers of invited girls. First, by developing the intervention in a systematic manner, we ensured a solid theoretical and empirical foundation for the intervention (cf. Bartholomew Eldridge et al., 2016). For decisions to be made about methods/applications that lack a solid ground of consensus in the research literature, we were able to pre-test the impact of alternative prototypes before finalizing and testing the full operational intervention.

Furthermore, according to IM, it is imperative that members of the target group are involved in the development of the intervention (Bartholomew Eldridge et al., 2016). However, currently, in many eHealth interventions, the design of the intervention is based on assumptions that are not validated with input from end-users. In fact, the importance of formative research and pretesting of materials is often being overlooked. The resulting intervention may therefore lack key features, and subsequent evaluations of the effectiveness of the interventions may be compromised (Cafazzo et al., 2009). Therefore, we applied a user-centered design by extensively involving mothers in the intervention development from the beginning to the end (Mao et al., 2005). This was done by conducting focus groups and online experimental pretests, in which we gathered feedback from representatives of the target group. This iterative process of development and feedback guided our attempt to gradually improve the solution we had to offer for reaching the intended objectives. In other words, erroneous or inconclusive decisions can thoughtfully be changed or reversed in order to prevent the final intervention from being at odds with the objectives set beforehand. An example illustrating this is our decision to remove a component targeting anticipated regret from the intervention as it clearly evoked resistance as shown by the focus groups. Based on the feedback from the focus groups (Step 4), we changed the method targeting anticipated regret (Step 3), and pretested the intervention again (Step 4). Thus, we moved back and forth between the steps. Furthermore, not only did we fine-tune the content of the intervention to the mothers' preferences, but also the design of the website was chosen by the mothers. Hence, we adapted the entire intervention to the requirements and preferences of the mothers. Next to maximizing the likelihood of success, using IM has made the 
process of intervention development explicit and transparent, providing a road map of the decision-making process and its main outcomes. This will suit the interpretation of strengths and weaknesses of the intervention when looking at the results from the outcome evaluation (Step 6) (Pot et al., 2017a). It also enables the owner to improve the intervention where necessary and others to replicate the steps described when developing a similar intervention for different populations and/or settings (Leerlooijer et al., 2011).

\section{Design rationales in eHealth}

Recently, it has been argued that eHealth researchers should publish descriptions of interventions and results from evaluation studies separately in order to gain a better understanding of what exactly is being evaluated, facilitate comparison between interventions, and extend the evidence base for the development of future interventions (Brendryen et al., 2013; Drozd et al., 2015). The current paper complies to this call and adds to the plea for systematic and detailed descriptions of design rationales in the eHealth field. Systematic descriptions may improve the quality of future systematic reviews that assess the link between design features and outcomes of an intervention (Brouwer et al., 2011; Gardner et al., 2010; Morisson et al., 2012). These reviews, in turn, can be used as a guide for eHealth researchers in designing future interventions with improved efficacy, reach, and user acceptability (Brendryen et al., 2013).

\section{Limitations}

Although we believe that using IM has greatly contributed to the intervention being effective in promoting HPV vaccination acceptability, we agree with other authors that IM is a complex and time-consuming process (Côte et al., 2008; Heinen et al., 2006; Van Kesteren et al., 2006). However, we are convinced that the development of the intervention was brought to a higher level by IM. Moreover, we believe that the experience we gained may improve efficacy of the process and make it less time-consuming in future intervention development. We can profit from this experience when developing similar interventions for a different population (e.g., an intervention promoting HPV vaccination among Dutch boys).

\section{Conclusion}

In this article, we provide a detailed, comprehensive description of how we systematically developed an intervention promoting HPV vaccination acceptability. Using IM led to an innovative and effective intervention using interactive Web-based computer-tailored education. This intervention blueprint will aid in interpreting the results of our evaluation studies. Moreover, it will ease comparisons of design rationales across interventions, and may provide leads for the development of other eHealth interventions. Overall, this paper 
adds to the plea for systematic reporting of design rationales constituting the process of developing interventions, and the development of a cumulative science of interventions in the eHealth field.

\section{Conflicts of Interest}

The authors declare that the research was conducted in the absence of any commercial or financial relationships that could be construed as a potential conflict of interest.

\section{Author Contribution Statement}

MP contributed to study conception and design, acquisition of data, analysis and interpretation of data, and drafting of manuscript. HVK, TP and RR contributed to study conception and design, interpretation of data and revising the manuscript critically. $\mathrm{AH}$ and HDM contributed to study conception and design and revising the manuscript critically. HVV critically revised the manuscript. All authors approved the final version of the manuscript to be published.

\section{Funding}

ZonMw (The Netherlands Organisation for Health Research and Development) supports this study (grant-number: 50-51515-98-259).

\section{Ethics Statement}

The study was approved by the Medical Ethical Committee (METC), the ethical committee of the VU Medical Center in Amsterdam. Informed consent was provided online for the online studies (i.e., the three experimental pretests, the online focus group and the RCT). For the focus groups, participants provided written consent. 



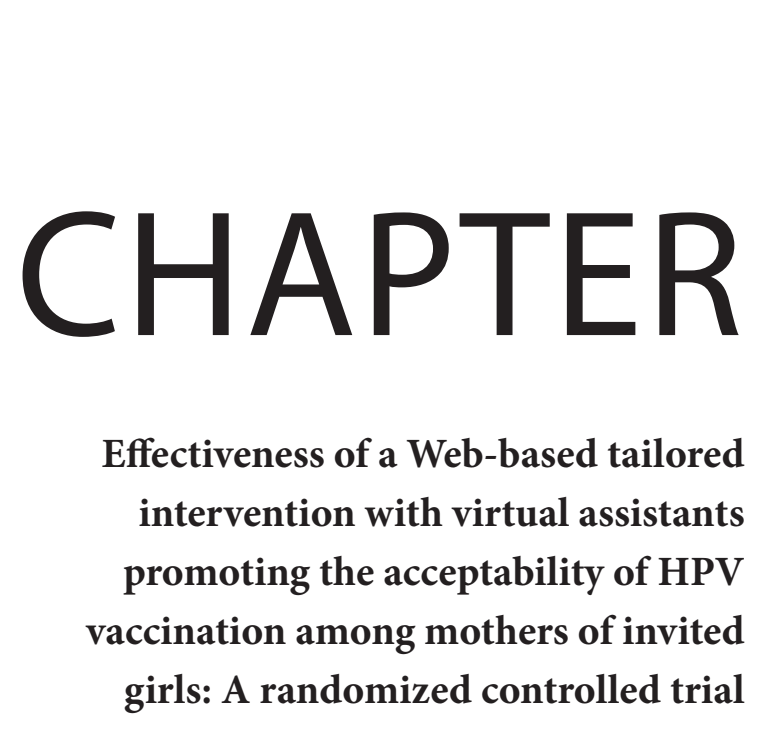

Pot, M., Paulussen, T.G.W.M., Ruiter, R.A.C., Eekhout, I., de Melker, H.E., Spoelstra, M.E.A., Van Keulen, H.M.

Journal of Medical Internet Research, 19(9), e312. 


\section{ABSTRACT}

In 2010, the human papillomavirus (HPV) vaccination was introduced in the Dutch National Immunization Program for 12-year-old girls, aiming to reduce the incidence of cervical cancer in women. HPV vaccination uptake turned out to be lower than expected: $61 \%$ versus $70 \%$, respectively. Mothers were shown to play the most important role in the immunization decision about this vaccination. They had also expressed their need for interactive personal information about the HPV vaccination over and above the existing universal general information. To improve the effectiveness of the existing education about the HPV vaccination, we systematically developed a Web-based tailored intervention with virtual assistants providing mothers of girls to be invited with tailored feedback on their decision making about the HPV vaccination. The aim of this study was to evaluate the effectiveness of the Web-based tailored intervention for promoting HPV vaccination acceptance by means of a randomized controlled trial (RCT). Mothers were recruited via the Dutch vaccination register (Praeventis) $(n=36,000)$ and three Web-based panels $(\mathrm{n}=$ $2,483)$. Those who gave informed consent $(\mathrm{N}=8,062)$ were randomly assigned to the control ( $\mathrm{n}=4,067)$ or intervention condition $(\mathrm{n}=3,995)$. HPV vaccination uptake, as registered by Praeventis once the HPV vaccination round was completed, was used as the primary outcome. Secondary outcomes were differential scores across conditions between baseline (before the provided access to the new tailored intervention) and follow-up (just before the first vaccination) regarding the mothers' degree of informed decision making (IDM), decisional conflict, and critical determinants of HPV vaccination uptake among which are intention, attitude, risk perception, and outcome beliefs. Intention-to-treat analysis $(\mathrm{N}=$ 8,062 ) showed a significant positive effect of the intervention on IDM, decisional conflict, and nearly all determinants of HPV vaccination uptake $(p<.001)$. No effect was found on uptake ( $p=.60)$. This may be attributed to the overall high uptake rates in both conditions. Mothers evaluated the intervention as highly positive, including the website as well as the virtual assistants that were used to deliver the tailored feedback. This computer-tailored intervention has the potential to improve HPV vaccination acceptability and IDM and to decrease decisional conflict among mothers of invited girls. Implications for future research are discussed. 


\section{INTRODUCTION}

Worldwide, cervical cancer is the third most common cancer in women (Ferlay et al., 2010). Persistent infection by the human papillomavirus (HPV) is the causative agent of cervical cancer (Schiffman \& Castle, 2003). In the Netherlands, yearly 750 new cases of cervical cancer are detected, of which 242 are with fatal consequences (Bruni et al., 2016), despite the presence of a national cervical cancer screening program for women aged 30 to 60 years (Braspenning et al., 2001). In 2008, the Health Council advised the Ministry to include the HPV vaccination for girls aged 12 years in the National Immunization Program (NIP) (Gezondheidsraad, 2008). Initial implementation started with a catch-up campaign in 2009 for girls aged 13 to 16 years. From 2010, new cohorts of 12-year-old girls have been invited by the NIP to receive the HPV vaccination on a yearly basis. The municipal health services organize local sessions for group-based HPV vaccination, usually at large venues. This restricts the opportunity for interaction between the parent and girl with the professional. The vaccination itself is given by young health professionals (i.e., medical doctors, nurses, or doctor's assistants). The vaccination is voluntary and is offered free of charge. Complete vaccination includes 2 injections with a 6-month interval.

So far, HPV vaccination uptake in the Netherlands has remained lower than expected: $61 \%$ uptake in 2016 while 70\% was targeted (Van Lier et al., 2016). Research has indicated that mothers play the most important role in decision making about the girls' immunization (Van Keulen et al., 2013a). Currently, the regular invitation for the HPV vaccination comprises an introduction folder and a link to a website providing universal information about HPV and HPV vaccination. However, research has already indicated that mothers feel more in need for interactive personal information about the HPV vaccination, over and above this universal information (Van Keulen et al., 2013a). To improve the existing educational strategy targeting HPV vaccination uptake, we developed a computer-tailored intervention with virtual assistants using the 6-step Intervention Mapping protocol for developing theory- and evidence-based health promotion interventions (Bartholomew Eldridge et al., 2016). The intervention was aimed at Dutch mothers of girls to be invited for the HPV vaccination in 2015 (i.e., girls born in 2002).

To date, only few tailored interventions to encourage HPV vaccination have been tested (Benett et al., 2015; Gerend et al., 2013; Grandahl et al., 2016; Hopfer, 2012). Three of these showed positive results. Hopfer (2012) found that HPV vaccination uptake doubled among participants who were exposed to a culturally tailored video, compared with controls. Gerend and colleagues (2013) found that, compared with general information, information tailored to the individual participant's perceived barriers increased HPV vaccination intention. Grandahl and colleagues (2016) found that an intervention delivered individually, 
that is face-to-face, by school nurses positively affected beliefs toward HPV prevention as well as vaccination uptake. However, to our knowledge, effective interventions promoting HPV vaccination that can reach large groups at relatively low costs (eg, Web-based tailored interventions) (Neville, O’Hara, \& Milat, 2009) do not exist yet.

Social cognitive determinants of the mothers' decision making about their daughters' HPV vaccination that appeared both relevant and changeable were selected as targets for developing the intervention (Bartholomew Eldridge et al., 2016). These included HPV vaccination-related intention, attitude, outcome beliefs, risk perception, anticipated regret, subjective norms, habit, relative effectiveness of the HPV vaccination, and self-efficacy (Pot et al., 2017b; Van Keulen et al., 2013a; Van Keulen et al., 2013b). These determinants appeared to account for large proportions of variance in the mothers' decision-making outcome (80-82\%) (Van Keulen et al., 2013a). Also, large proportions of the mothers do not actively acquire and process information about the pros and cons of the HPV vaccination and feel ambivalent about their decision (Van Keulen et al., 2013a; Van Keulen et al., 2013b). This indicates that these decisions are based on rather unstable grounds, which makes them vulnerable for arguments challenging their initial attitudes and/or intention. Because informed decision making (IDM) is expected to make mothers less vulnerable for counterarguments, this was also chosen as a relevant intervention target. According to Marteau and colleagues (2001), an informed decision is based in sufficient and relevant knowledge, and in the congruence between the person's values (i.e., their attitude toward the HPV vaccination) and the behavioral outcome (i.e., whether mothers had their daughters vaccinated against HPV). Consequently, knowledge was also targeted by the intervention. In addition, decisional conflict was selected as a target as this appears strongly related to IDM, with the possibility that decisional conflict may arise when feeling uninformed (O’Connor, Jacobsen, \& Stacey, 2002).

The aim of this study was to assess the effectiveness of the Web-based tailored intervention with virtual assistants on HPV vaccination uptake among the participants daughters (primary outcome). Secondary outcomes were the mothers' degree of IDM, decisional conflict, and the social cognitive determinants of decision making about the daughters' HPV vaccination uptake (e.g., attitude, intention, and beliefs). When compared with the control condition, significantly positive effects were expected in the intervention condition with respect to HPV vaccination uptake, social cognitive determinants of the mothers' decision making about the vaccination, levels of informed decision making, and levels of decisional conflict. 


\section{METHODS}

\section{Participants}

Mothers were randomly recruited from Praeventis, the Dutch National Immunization Register, and three Internet panels. The latter was to assure a high response rate (Van Keulen et al., 2013a). This gave us the opportunity to assess differential effects of the intervention under (1) more controlled conditions (i.e., panel sample) and (2) more naturalistic conditions as will be the case when the intervention has become part of the national implementation strategy (i.e., Praeventis sample). This provided a basis for inferences concerning the intervention's efficacy and effectiveness (Ernst \& Pittler, 2006). This is also why we did not reward the mothers from the Praeventis sample. The panel members received a small financial reimbursement for each survey that they completed (2-3 euros per survey). The amount of money received was panel specific. Extra financial reimbursement was provided to those in the intervention condition who completed all surveys (1-3 euros extra). In total, the participants in the control group could receive between 4 and 6 euros (completing baseline and follow-up), whereas participants in the experimental group could receive between 5 and 9 euros (completing baseline, intervention, and follow-up). Panel members were pre-stratified by region to ensure geographic diversity.

\section{Power calculation}

Power analyses indicated that 1,200 mothers per sample (i.e., Praeventis and panels) were needed at baseline (600 per arm) to detect a 10\% difference in HPV vaccination uptake between the intervention and control group, and small effects on the continuous secondary outcomes (Cohen's $d=0.10-0.30$ ), with a power of 0.80 , a two-sided alpha of .05 , and an expected dropout of $30 \%$ at the last survey. Given previous experiences (Van Keulen et al., 2013a; Van Keulen et al., 2013b), a response rate of 3\% was expected in the Praeventis sample. A total of 36,000 mothers were initially invited to participate via Praeventis and 1,200 mothers via the Internet panels.

\section{Design}

This study, approved by the Ethical Committee of the VU Medical Center in Amsterdam, was conducted between January 2015 and March 2015. Effectiveness was evaluated by a 2-arm randomized controlled trial (RCT). Intervention effects on HPV vaccination uptake were assessed objectively using Praeventis. Because invited girls were given the opportunity to catch up on their missed HPV vaccinations, complete data on uptake were only available 18 months after baseline (i.e., July 2016). The effects on secondary outcomes were examined by two Web-based surveys; at baseline, just before they had access to the experimental education, and at follow-up, just before they received the first HPV vaccination (time 
intervals around 2 months). Participants in the intervention condition were invited to visit the Web-based intervention between baseline and follow-up. Participants in both arms had access to the universal information about the HPV vaccination as part of the regular invitation for the HPV vaccination.

\section{Procedure}

An invitation letter to participate in the study was sent in January 2015 by postal mail to the Praeventis sample and by email to the panel sample. This letter included information about the study, a link to a secured website, and a unique code for entrance to the baseline survey. The same code was used for entering the follow-up survey and for gaining access to the tailored intervention (only participants in the intervention condition). Securing the website and providing unique codes was done to reduce the risk of spillover effects. The mothers in the intervention condition were explicitly requested not to share the link with others. On the website, the participants were assured of their privacy, confidentiality, and security in handling their responses and were informed that they could withdraw from participation at any time. Participants were then asked to provide informed consent and to give us permission to derive their daughters' HPV vaccination status from Praeventis. After having provided informed consent, participants were randomly assigned to either the control or intervention condition. A reminder was sent one week after the first invitation. One week after the reminder, participants in the intervention condition received an email inviting them to visit the Web-based tailored feedback. Two weeks after this invitation, a reminder was sent. The website could be visited until the invitation to complete the followup questionnaire, 8 weeks after the initial invitation for the baseline questionnaire. All participants were given 2 weeks to complete the follow-up survey; a reminder was sent after the first week. These timelines (see Figure 5.1) were chosen as these fit with the standard procedures for the HPV vaccination in the Netherlands.

\section{Intervention}

The intervention consisted of a website providing mothers with tailored feedback from 2 virtual assistants, one being visualized in Figure 5.2. In Appendix E, a selection of screenshots of the website is presented. Computer-tailoring was the basic method for change and fitted the outcome of a previously conducted needs assessment indicating that the mothers preferred personalized feedback (Van Keulen et al., 2010). Tailoring is a health communication strategy in which messages are individualized to the person's preferences and needs (Kreuter \& Wray, 2003). Meta-analyses have shown computer-tailored interventions to be more effective than universal interventions in achieving behavioral change outcomes (e.g., Krebs, Prochaska, \& Rossi., 2010; Lustria et al., 2013). Examples of change techniques that were used in addition to tailoring were consciousness raising 
(targeting risk perception) (Prochaska, Wright, \& Velicer, 2008), belief selection (targeting beliefs) (Fishbein \& Ajzen, 2010), active learning (targeting knowledge retention) (Petty, Barden, \& Wheeler, 2009), and motivational interviewing (targeting decisional conflict and attitude change) (Miller \& Rollnick, 2002). Before completing the concept intervention, we experimentally pretested three different intervention components (Whittingham et al., 2008a; Whittingham et al., 2008b) and conducted three focus groups for pretesting the prototype's feasibility.
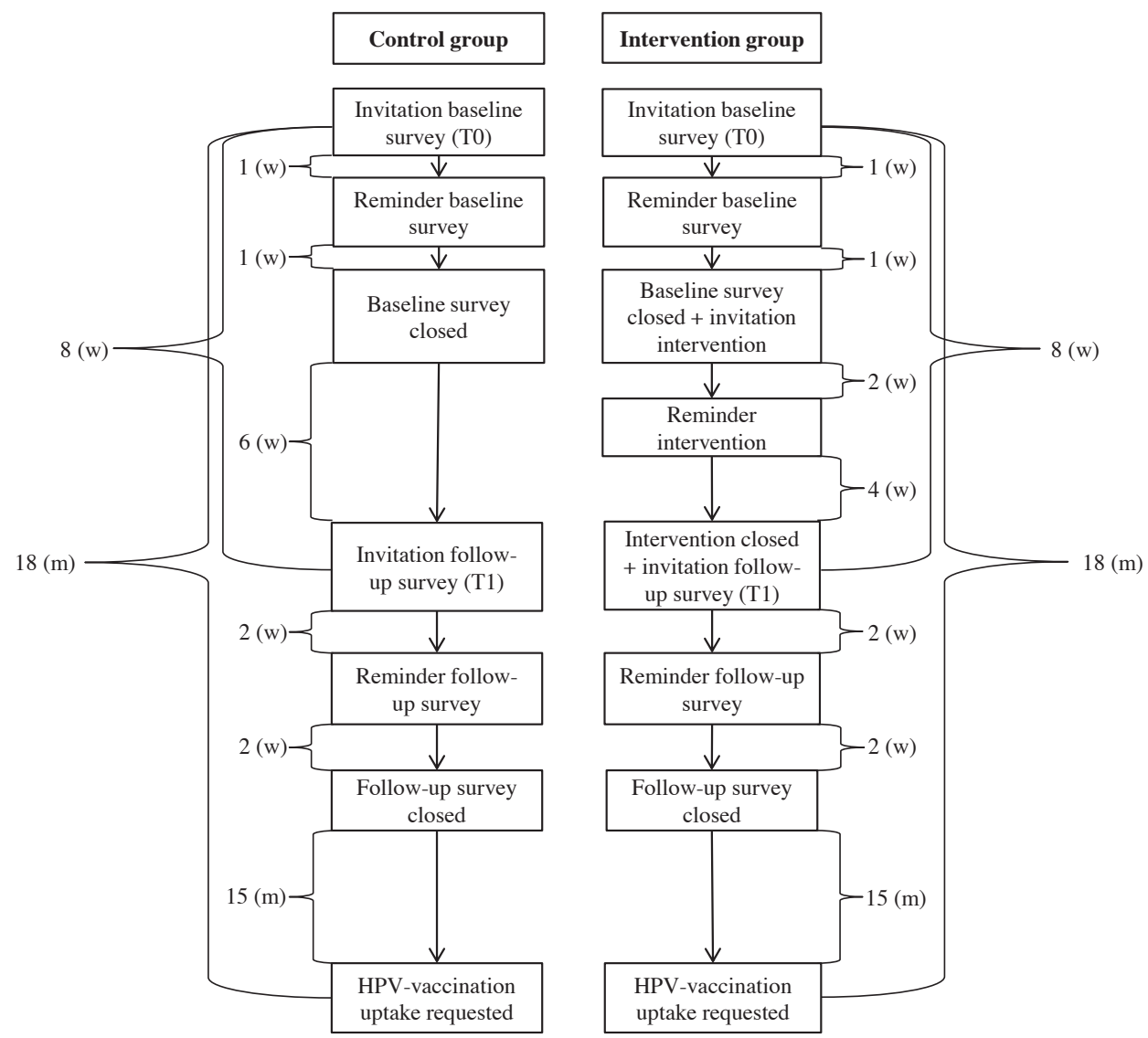

Figure 5.1 Timeline of data collection for the control and intervention group.

Also, innovative was the use of 2 virtual assistants for delivering the tailored feedback; a mother- and doctor-like assistant. They provided opportunities for two-way interactions and for creating a highly personal experience. The added value of using a virtual assistant 
over a text and/or picture-based website is that it improves information recall (Beun, de Vos, \& Witteman, 2003), transfer of learning (Atikinson, 2002), amount of learning (Baylor \& Kim, 2004), self-efficacy expectations, literacy, and behavioral change (Blanson Henkemans et al., 2009; Blanson Henkemans et al., 2008; Jin, 2010).

The website consisted of four menu options: (1) two-sided information about the HPV vaccination, (2) a decisional balance, (3) practical background information, and (4) frequently asked questions (see Appendix E). In the first menu, mothers were able to collect tailored information about the HPV vaccination such as information about the risk of contracting an HPV infection, which may cause cervical cancer, as well as the risks and effectiveness of the HPV vaccine. In the second menu, a decisional balance gave mothers the opportunity to weigh their perceived pros and cons to balance the mothers' position toward vaccinating versus not vaccinating the daughter (see Figure 5.2). In the third menu, mothers received practical information such as how and where to get the HPV vaccination and how to talk to their daughter and/or partner about the HPV vaccination.

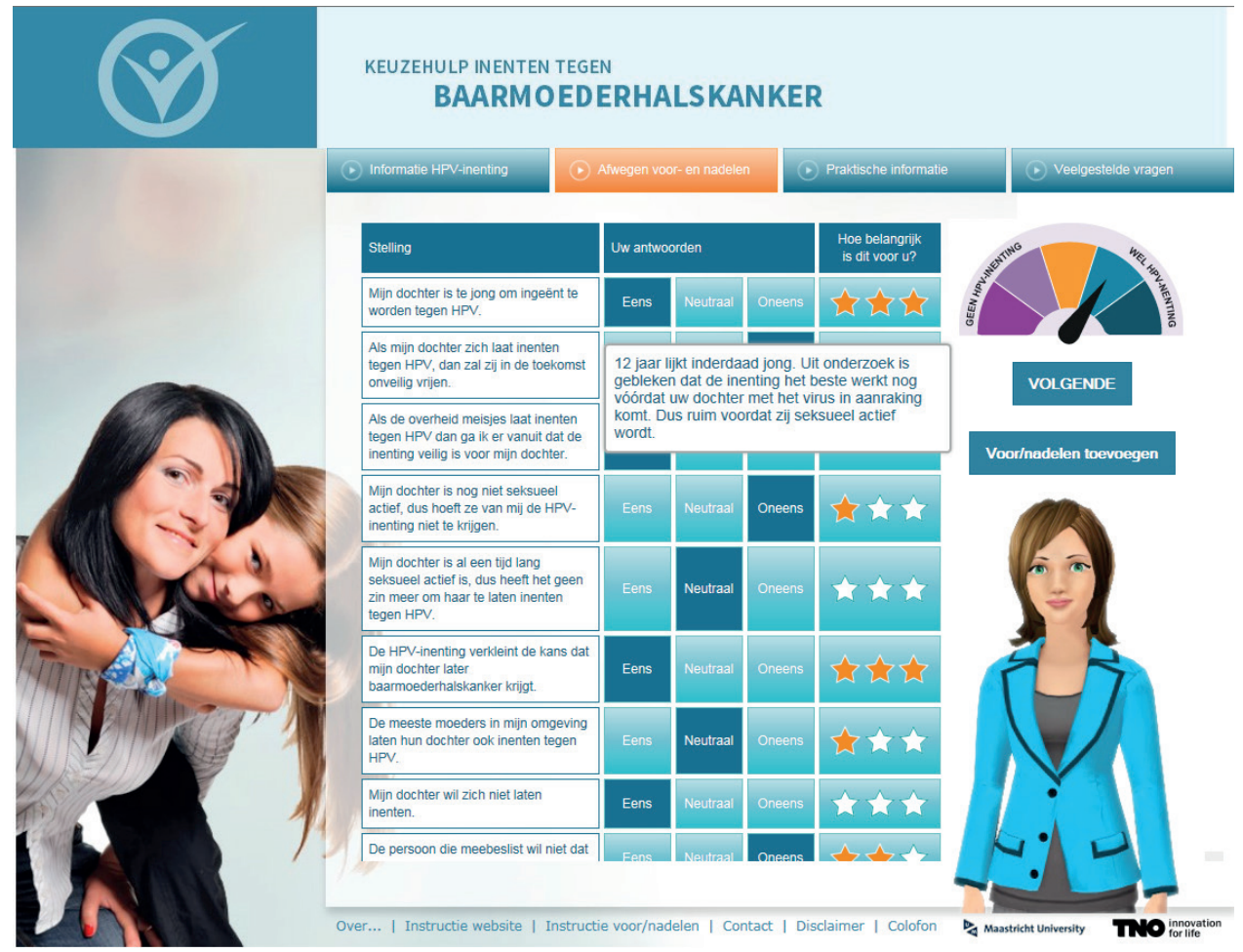

Figure 5.2 Screenshot of the decisional balance and the mother-like virtual assistant on the website. 


\section{Outcome measures}

\section{Primary outcome: $H P V$ vaccination uptake}

HPV vaccination uptake was derived from Praeventis, which was registered as having received no, 1, or 2 injections. We dichotomized HPV vaccination uptake into having received no HPV injection $(0=$ not vaccinated $)$ versus having received 1 or $2 \mathrm{HPV}$ injections ( 1 = vaccinated), as data showed that the determinants of HPV vaccination contrasted in these groups contrasted the most.

\section{Secondary outcomes: IDM and decisional conflict}

According to Marteau and colleagues (2001), a choice is considered to be informed when people have sufficient and relevant knowledge (knowledge) and when the person's values (attitude) and behavior match. As such, IDM is usually expressed dichotomously (e.g., see Van der Pal, Otten, \& Detmar, 2010). However, as we think the selection of the cut-off points is somewhat arbitrary, yet critical for the outcome (Atman \& Royston, 2006), we also constructed a continuous measure for IDM. Post hoc analyses showed the correlation to be high between the two (Spearman $\rho=.78$ ). Both scores were derived from the Multidimensional Measure of Informed Choice (Marteau, Dormandy, \& Michie, 2001; Michie, Dormandy, \& Marteau, 2002; Van der Pal, Otten, \& Detmar, 2010).

By constructing the dichotomous IDM, the mother was classified as an informed decision maker, if (1) she had sufficient knowledge and (2) her attitude was consistent with the behavior (HPV vaccination uptake). Knowledge was considered to be sufficient when it was higher than the baseline mean score. A decision was considered to be consistent when the mother's attitude was positive (higher than 4 on a 7 -point scale) and the daughter was vaccinated, or when her attitude was negative (lower than 4 on a 7-point scale) and her daughter was not vaccinated. Any other combination was categorized as an uninformed decision.

By constructing the continuous measure of IDM, we first recoded the attitude scale into a scale ranging from -3 (extremely negative) to 3 (extremely positive). Consistency (ranging from -3 to 3 ) resulted from multiplying this attitude score with uptake $(-1=$ no injection vs $1=1$ or 2 injections). Then, the resulting consistency score was recoded from 0 (least consistent) to 6 (most consistent). Next, knowledge ( -8 to 8 , see Table 5.1 ) was recoded into a scale ranging from 0 (least knowledgeable) to 8 (most knowledgeable); the original scores below 0 were all recoded to 0 . Finally, the continuous scale for IDM was computed by multiplying knowledge (range $0-8$ ) with consistency (range $0-6$ ), resulting in a scale ranging from 0 (not/least informed) to 48 (most informed). 
Decisional conflict was measured using the "Uncertainty" subscale of the Decisional Conflict Scales (O'Connor, 1995), which included three 7-point scaled items (e.g., "as regards to the HPV vaccination, I felt sure about my choice) $(1=$ completely disagree to $7=$ completely agree). Internal consistency was high (Cronbach $\alpha=.94)$.

\section{Determinants of HPV vaccination uptake}

Composite scores were computed for determinants of HPV vaccination uptake in case the scaled items showed internal consistency (Cronbach $\alpha>.60$ or Pearson $r>.64$ ). See Table 5.1 for an overview of the primary and secondary outcome measures. All scores on scaled items were averaged into a scale because they showed sufficient internal consistency (Cronbach $a$ $\geq .78 /$ Pearson $r \geq .64$ ); Cronbach alpha was used for scales consisting of more than 2 items, whereas Pearson $r$ was used for scales consisting of 2 items; items with an (R) were reverse coded.

\section{Socio-demographics}

Socio-demographics were modeled as background variables (i.e., age, educational level, country of birth, and religion). Level of education referred to the highest level the mother had completed. Educational level was classified into low (less than secondary or vocational education), intermediate (secondary through pre-university education) or high (professional or university education) (Van Keulen et al., 2013a; Van Keulen et al., 2013b). Country of birth was dichotomized into "Netherlands" versus "other," as in our sample only $562(6.97 \%)$ of $8,062(100 \%)$ mothers appeared to be born in a country other than the Netherlands. Religion was measured by asking the mothers about their religious convictions (Protestant, Roman Catholic, Muslim, Jewish, Buddhist, Hindu, other, or no religion). This was later classified as "Protestant" versus "not Protestant" as Protestants most refrain from vaccination compared with other religious or nonreligious groups in the Netherlands (Van Keulen et al., 2013a; Van Keulen et al., 2013b).

\section{Subjective program evaluation and objective program use}

Subjective program evaluation was assessed at follow-up for mothers in the intervention condition by asking them to evaluate the website and the virtual assistants on a 10-point scale; the higher the score, the more positive the evaluation. Objective program use was evaluated by computer logs assessing the number of visits and amount of time logged in per session. If participants logged in more than once, these were summed. 


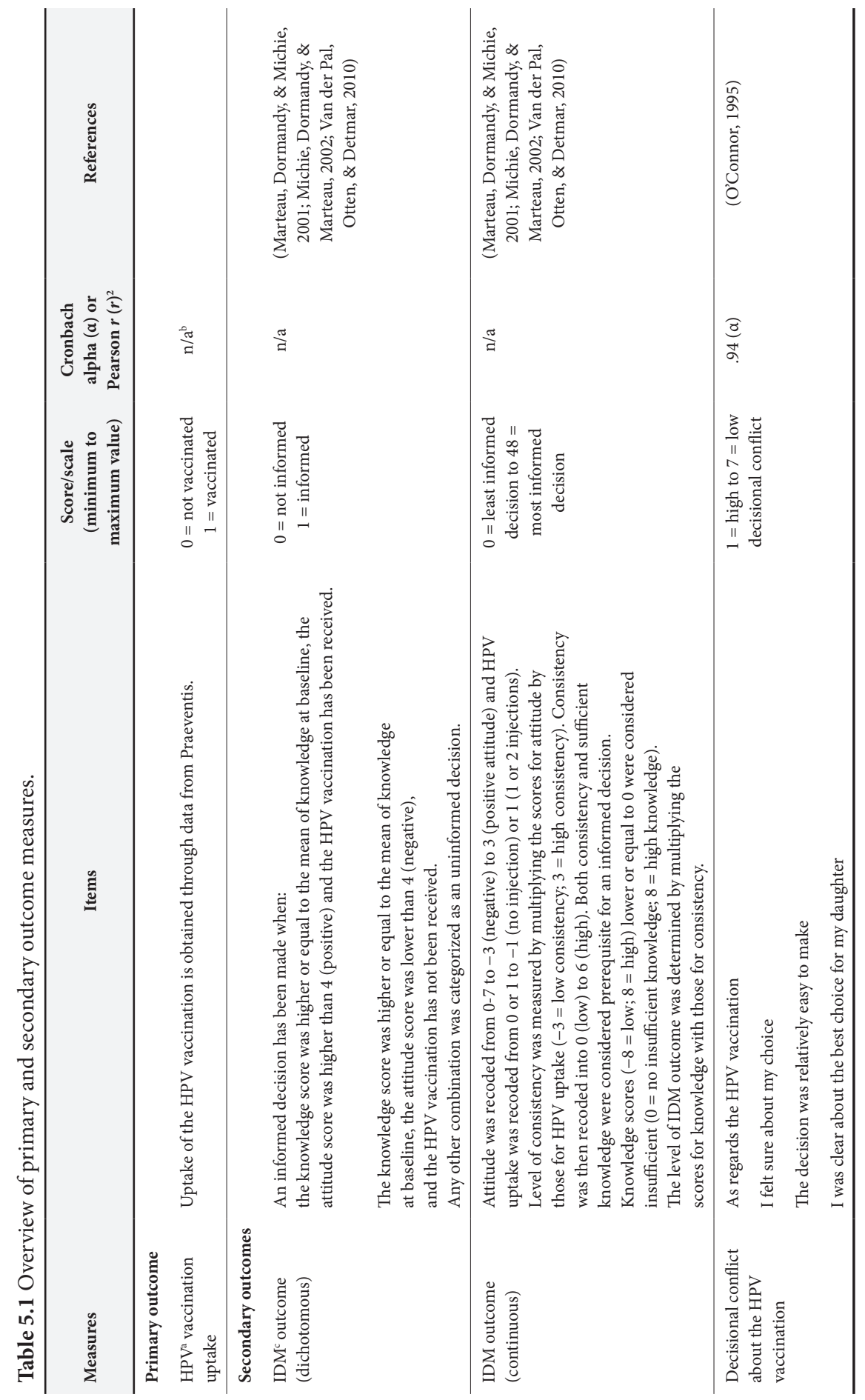




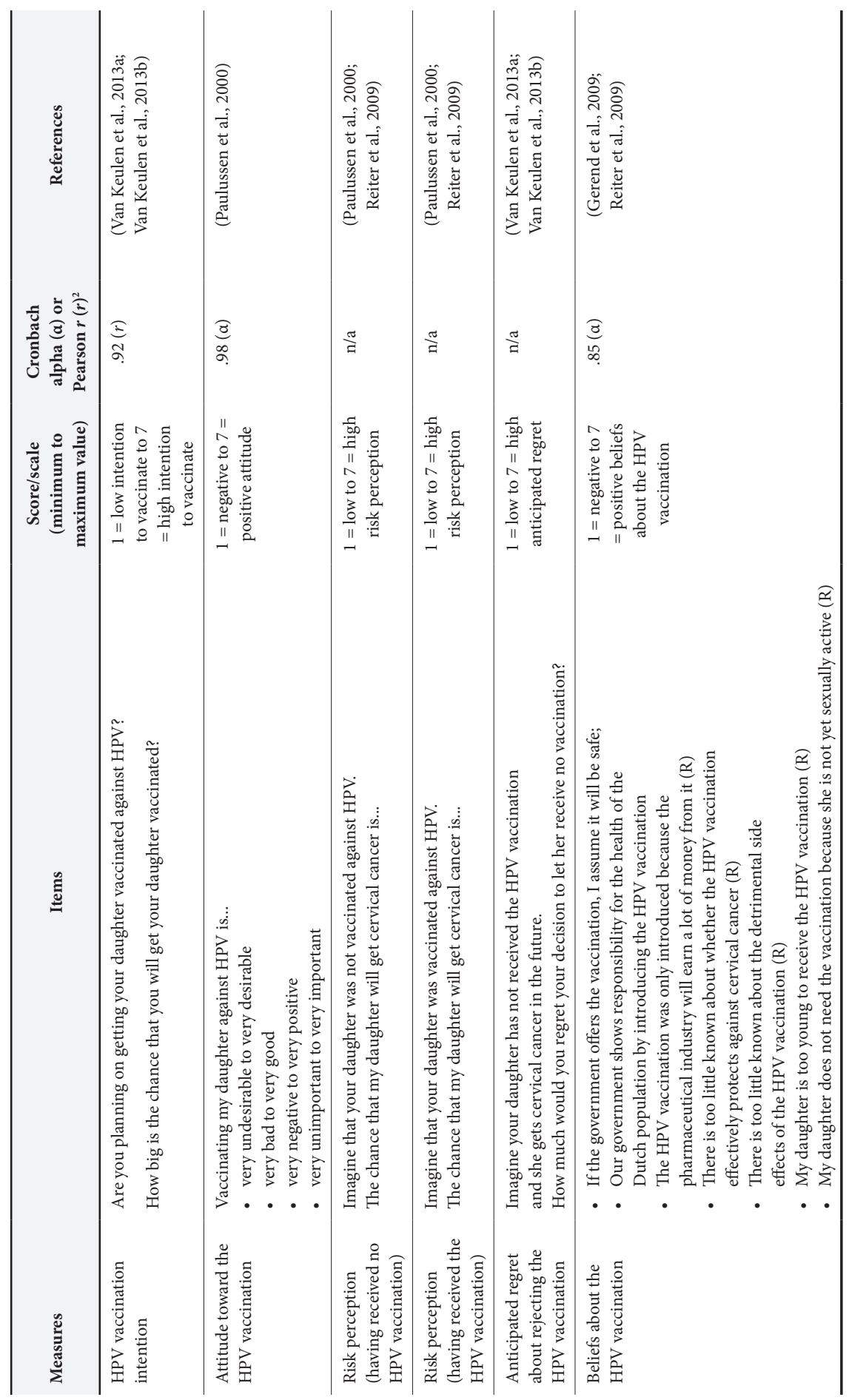




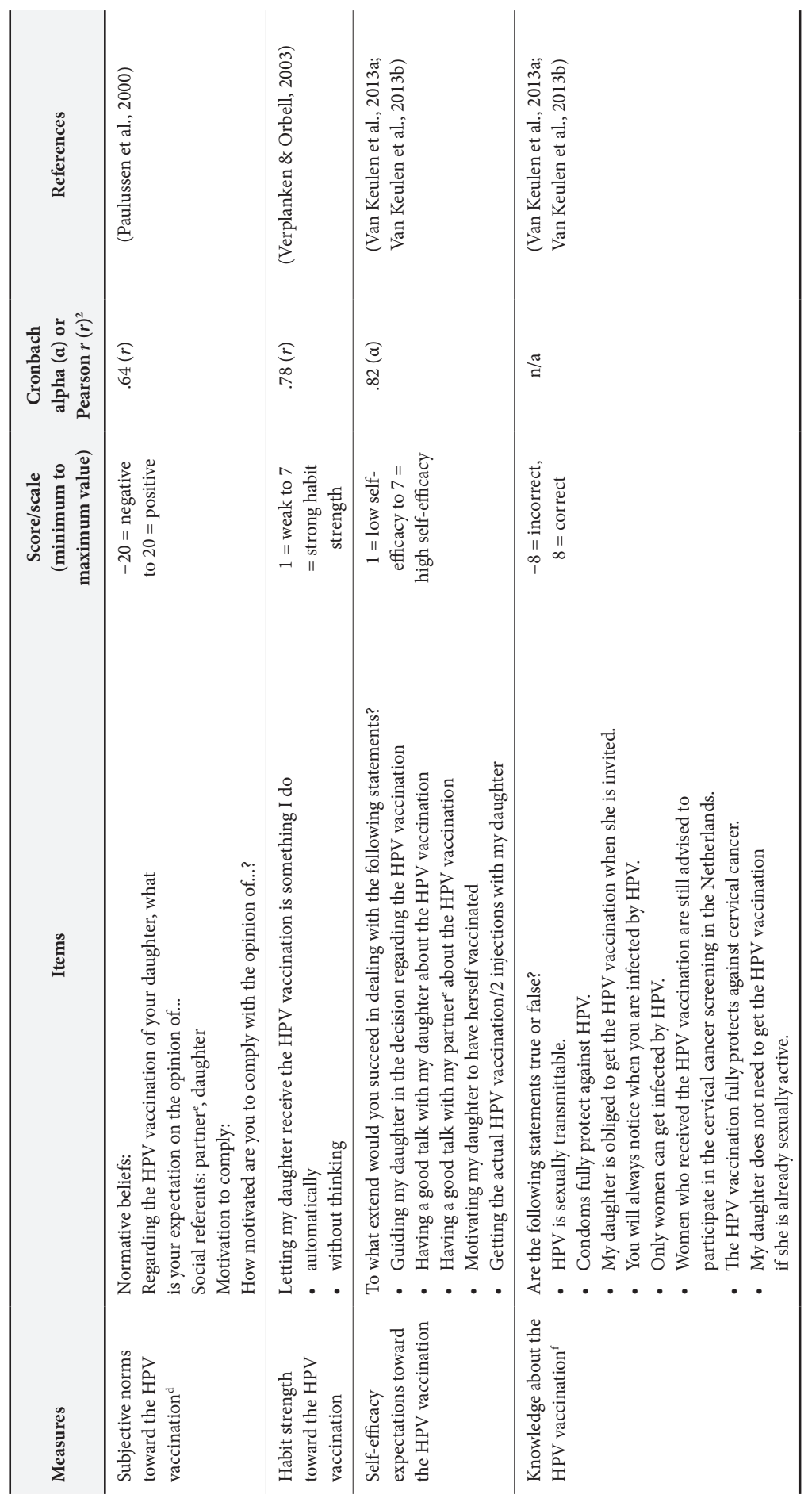




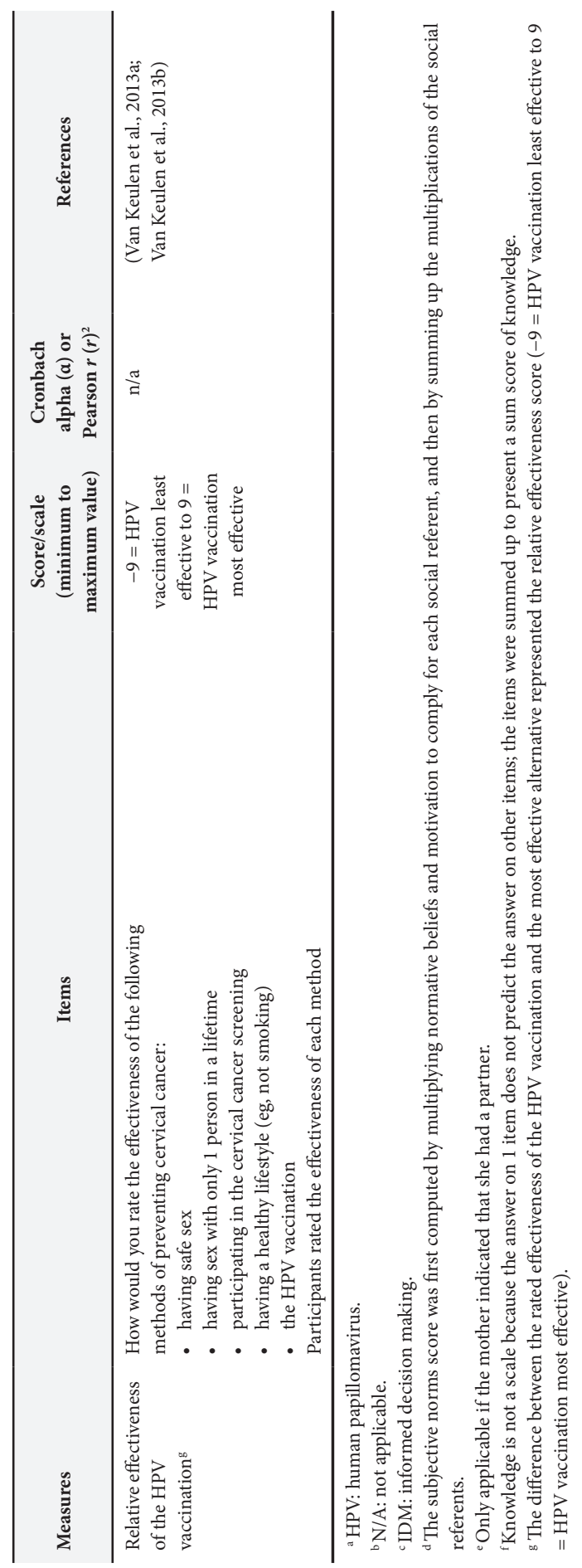




\section{Statistical Analyses}

Descriptive statistics were used to describe the baseline sample. For analyzing the effects of the intervention, we used intention-to-treat (ITT) instead of complete case analysis. By using ITT, power increases while the risk of bias possibly caused by selective dropout decreases (Van Buuren, 2012). To deal with missing data, we applied multiple imputation by chained equations (Van Buuren, 2012; White, Royston, \& Wood, 2011). There were 15 imputed datasets generated using the predictive mean matching algorithm in Statistical Package for the Social Sciences (SPSS, IBM Corp). The results from the imputed datasets were pooled together using Rubin's rules (Rubin, 1987). Convergence of the imputations was checked by inspecting the iteration plots.

Intervention effects were examined by logistic and linear regression analyses (for dichotomous and continuous variables, respectively) by using the outcome at the follow-up as the criterion and the outcome-score at baseline and condition as the independent variables (Van Breukelen, 2006). In view of multiple testing, an effect was considered significant when $p<.003$ (Bonferroni corrected alpha $=.05 / 15$ factors). The odds ratio was used as an indicator for effect size (Bonferroni corrected alpha $=.05 / 14$ factors). Effect sizes of the linear regressions were calculated in $\mathrm{R}$ ( $\mathrm{R}$ Development Core Team) ( $\mathrm{R}$ Development Core Team, 2009) using Cohen $f^{2}\left(R^{2}\right.$ including the outcome at baseline and condition/1- $R^{2}$ only including the outcome at baseline). These were interpreted as $0.02=$ small, $0.15=$ medium, $0.35=$ large (Cohen, 1988). Complete case analyses were performed as a sensitivity check for substantial differences with the results based on ITT.

Furthermore, we performed exploratory moderation analysis (Bonferroni corrected alpha $=.05 / 15)$ to examine differences in effects regarding socio-demographics (i.e., age, country of birth, education level, and religion) and sample (i.e., Praeventis vs panels) by including a two-way interaction term (e.g., condition $\times$ sample) in each of the aforementioned regression analyses (Fayers \& King, 2009). In addition, we explored whether intention (at baseline) moderated the effects found in the primary and secondary outcomes. For this, intention was divided into three subgroups: (1) mothers with a negative intention (scores below half a standard deviation $(S D)$ below the centered mean score of intention at baseline), (2) mothers who were hesitating (scores between half an SD below and above the centered mean of intention at baseline), and (3) mothers with a positive intention (scores more than half an SD above the centered mean score). Finally, subjective evaluations and objective use of the program were assessed by using descriptive analysis. IBM statistical package SPSS version 23 was used for analyzing the data (IBM Corp, 2013). 


\section{RESULTS}

\section{Response rates and attrition}

We invited 36,000 participants via Praeventis and 2,483 via the panels. A flow diagram of the recruitment and response is shown in Figure 5.3. From the 9,124 participants who were initially randomized at T0, 8,593 (94.18\%; 4,277 in the intervention group and 4,316 participants in the control group) completed the baseline questionnaire, whereas 4,678 (51.27\%; 2,197 in the intervention group and 2,481 participants in the control group) completed the follow-up questionnaire 8 weeks later (T1). Dropout analysis showed significantly more dropout in the Praeventis sample. There was also selective nonresponse with regard to condition (i.e., more dropout in the intervention condition), sociodemographics (i.e., more dropout in those not born in the Netherlands, and in those low in education), HPV vaccination uptake (more dropout in mothers having a daughter not being vaccinated), and secondary outcomes (i.e., more dropout in mothers with low levels of IDM, risk perception, and self-efficacy and in mothers with high attitude scores). In total, 1,067 participants were excluded (564 in the intervention group and 503 participants in the control group), as they did not meet the inclusion criteria (i.e., being a mother of a daughter born in 2002 and aged 24-62 years) or were found to be duplicates across the two samples. The final sample for ITT analysis consisted of 8,062 mothers: 3,995 mothers in the intervention condition versus 4,067 in the control condition.

\section{Sample description}

See Table 5.2 for the sample description. As there were no data available on sociodemographics of the population from which the sample was derived (i.e., Dutch mothers of girls aged 12 years in the Praeventis database), we were unable to assess the representativeness of the study sample. The mean age of mothers was 43.64 years $(S D=4.25)$. On average, mothers had a positive intention toward the HPV vaccination of their daughter at baseline $(M=5.35, S D=1.69)$. Compared with the national HPV vaccination uptake, uptake was higher in the study sample ( $\mathrm{n}=59,866 ; 60.98 \%$ vs $\mathrm{n}=5,880 ; 72.93 \%$, respectively). 


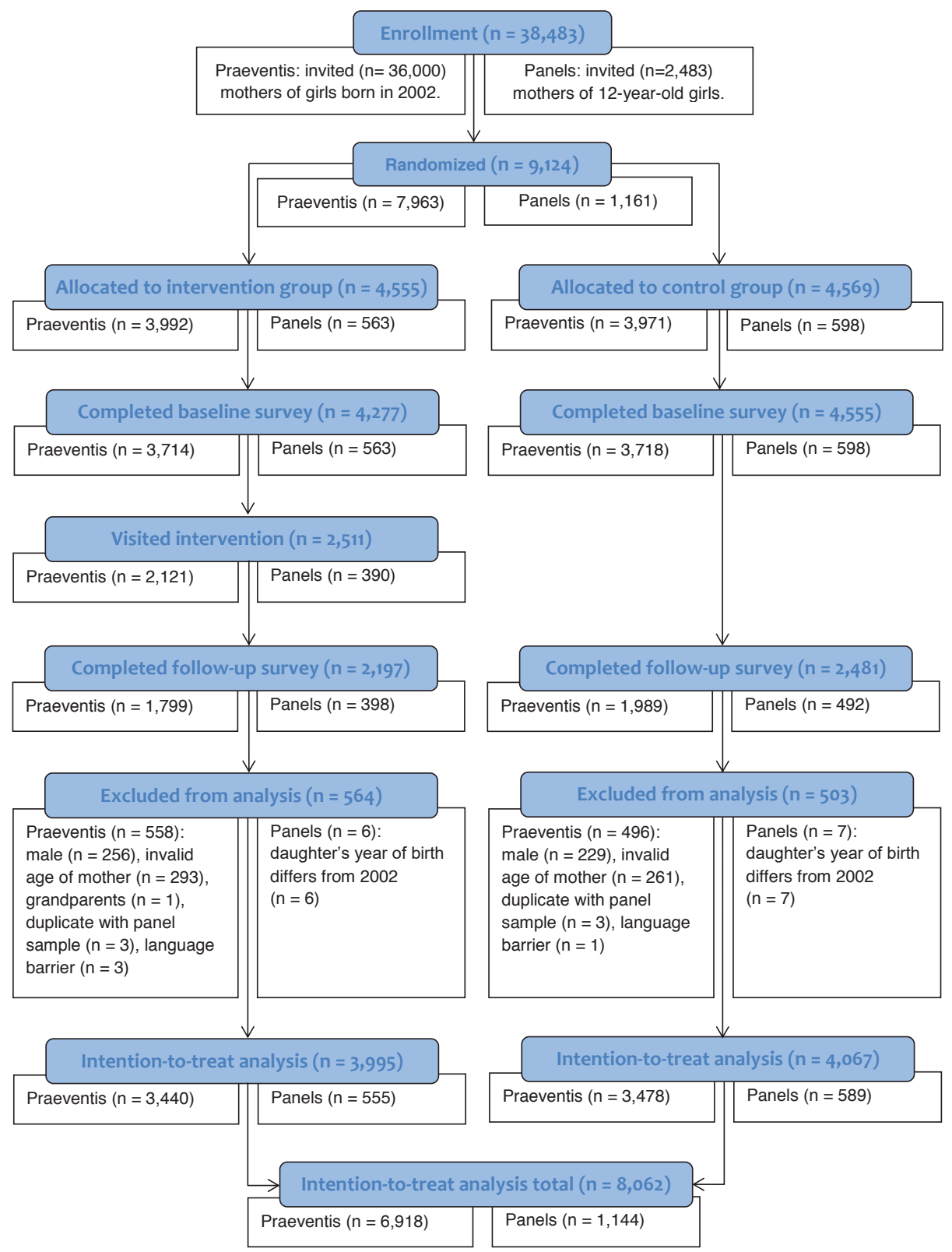

Figure 5.3 Flow diagram of the recruitment and response of study participants. Notes: (1) Participants could be excluded based on multiple criteria (e.g., a male with an invalid age). Therefore, the total of number of Praeventis participants excluded differed from the sum of separate criteria for exclusion. (2) In order to assess the intervention's effectiveness (Praeventis sample) versus efficacy (panel sample), the recruitment and response is displayed per sample within each condition. 
Table 5.2 Sample description ( $\mathrm{N}=8,062)$. In case of missing values, the number of missing values $\left(\mathrm{N}_{\text {missing }}\right)$ was presented. By reporting 2 decimal points for the percentages, summing the percentages for each category up may differ from $100 \%$.

\begin{tabular}{|c|c|c|c|}
\hline Variables & $\begin{array}{c}\text { Intervention } \\
\left(\mathrm{n}_{\text {total }}=3,995\right), \mathrm{n}(\%)\end{array}$ & $\begin{array}{c}\text { Control } \\
\left(\mathrm{n}_{\text {total }}=4,067\right), \mathrm{n}(\%)\end{array}$ & $\begin{array}{c}\text { Total } \\
\left(\mathrm{N}_{\text {total }}=\mathbf{8 , 0 6 2}\right), \mathrm{n}(\%)\end{array}$ \\
\hline Age in years, $\mathrm{n}(\%)$ & $43.70(4.27)$ & $43.58(4.22)$ & $43.64(4.25)$ \\
\hline $\begin{array}{c}\text { Country of Birth, } \mathrm{n}(\%) \\
\text { The Netherlands }\end{array}$ & $\begin{array}{c}\mathrm{N}_{\text {missing }}=4(0.10) \\
3.715(92.99)\end{array}$ & $\begin{array}{c}\mathrm{N}_{\text {missing }}=4(0.10) \\
3,777(92.87)\end{array}$ & $\mathrm{N}_{\text {missing }}=8(0.10)$ \\
\hline Other & $276(6.91)$ & $286(7.03)$ & $562(6.97)$ \\
\hline Religion, n (\%) & $\mathrm{N}_{\text {missing }}=7(0.18)$ & $\mathrm{N}_{\text {missing }}=6(0.15)$ & $\mathrm{N}_{\text {missing }}=13(0.16)$ \\
\hline Protestant & $753(18.85)$ & $737(18.12)$ & $1,490(18.48)$ \\
\hline Not protestant & $3,235(80.98)$ & $3,324(81.73)$ & $6,559(81.36)$ \\
\hline Educational level, n (\%) & $\mathrm{N}_{\text {missing }}=4(0.10)$ & $\mathrm{N}_{\text {missing }}=3(0.07)$ & $\mathrm{N}_{\text {missing }}=7(0.09)$ \\
\hline Low & $588(14.72)$ & $540(12.28)$ & missing (13.99) \\
\hline Middle & $1,736(43.45)$ & $1,735(42.66)$ & $3,471(43.05)$ \\
\hline High & $1,660(41.55)$ & $1,786(43.91)$ & $3,446(42.74)$ \\
\hline $\mathrm{HPV}^{\mathrm{a}}$ vaccination uptake, $\mathrm{n}(\%)$ & $2,923(73.17)$ & $2,957(72.71)$ & $5,880(72.93)$ \\
\hline
\end{tabular}

${ }^{\mathrm{a}} \mathrm{HPV}$ : human papillomavirus.

\section{Intervention effects on primary and secondary outcome(s)}

In Table 5.3, an overview of the intervention effects on the primary and secondary outcomes is given. A higher mean score means a higher X (e.g., more positive attitude) except for decisional conflict; here, a higher mean score means less decisional conflict If an odds ratio (OR) is higher than one, this means that the higher the score on a factor, the higher the outcome of IDM or the higher the chance of the daughter being vaccinated. If an OR is less than one, this means that the higher the score on a factor, the lower outcome of IDM or the lower the chance of the daughter being vaccinated. ITT analyses showed that there was no effect of the intervention on HPV vaccination uptake (odds ratio, OR $=1.03$, $p=.60)$. The intervention had a significant positive effect on all secondary outcomes $(p$ $<.001)$, except for risk perception when not vaccinated, anticipated regret, and self-efficacy ( $p=.01 ; p=.01 ; p=.03$, respectively). Compared with the control group, at follow-up, mothers in the intervention group were more informed (dichotomous measure: $\mathrm{OR}=1.28$, $p<.001$; continuous measure: $\beta=1.72, p<.003)$, experienced less decisional conflict $(\beta=.21$, $p<.003)$, were more intended to vaccinate their daughter $(\beta=.18, p<.004)$, had a more positive attitude toward vaccinating their daughter $(\beta=0.15, p<.004)$, had more positive beliefs (e.g., beliefs about the safety and effectiveness of the HPV vaccination; $\beta=.12, p$ $<.003)$, had a lower risk perception when they imagined that their daughter was vaccinated $(\beta=-.11, p<.003)$, perceived more positive subjective norms $(\beta=.82, p<.003)$, reported a higher relative effectiveness $(\beta=.46, p<.003)$, and had more knowledge $(\beta=.35, p<.003)$. Effect sizes were small (see Table 5.3). Results from complete case analyses were similar, except for an additional effect of the intervention on anticipated regret and self-efficacy. 
Table 5.3 Effects of the intervention on the outcome measures according to intention-to-treat analyses $(\mathrm{N}=8,062)$.

\begin{tabular}{|c|c|c|c|c|c|c|c|}
\hline & \multirow[t]{2}{*}{ Outcome } & \multicolumn{2}{|c|}{$\begin{array}{c}\text { Control }(\mathrm{N} \\
=4,067), \\
\text { mean }(S D) \text { or } \\
\text { percentage }(n)\end{array}$} & \multicolumn{2}{|c|}{$\begin{array}{c}\text { Intervention } \\
(\mathrm{N}=3,995) \text {, } \\
\text { mean }(S D) \text { or } \\
\text { percentage }(\mathrm{n})\end{array}$} & \multirow[t]{2}{*}{$\begin{array}{c}\text { Beta } \\
\text { (standard } \\
\text { error) }\end{array}$} & \multirow[t]{2}{*}{$\begin{array}{c}\text { Cohen } \\
f^{2} \text { or } \\
\text { OR }\end{array}$} \\
\hline & & Pretest & Posttest & Pretest & Posttest & & \\
\hline \multicolumn{8}{|l|}{ Primary outcome } \\
\hline \multirow[t]{2}{*}{$\begin{array}{l}\text { HPV vaccination } \\
\text { uptake }^{\mathrm{a}}\end{array}$} & $\begin{array}{l}\text { Has received no HPV injection } \\
\text { (reference), percentage (n) }\end{array}$ & & $\begin{array}{c}1,106 \\
(27.19)\end{array}$ & & $\begin{array}{c}1,066 \\
(26.67)\end{array}$ & & \\
\hline & $\begin{array}{l}\text { Has received one or two HPV } \\
\text { injections, percentage (n) }\end{array}$ & & $\begin{array}{c}2,961 \\
(72.81)\end{array}$ & & $\begin{array}{c}2,929 \\
(73.32)\end{array}$ & $.03(.05)^{\mathrm{b}}$ & 1.03 \\
\hline \multicolumn{8}{|l|}{ Secondary outcomes } \\
\hline \multirow[t]{2}{*}{ IDM: dichotomous } & $\begin{array}{l}\text { Not informed (reference), } \\
\text { percentage }(\mathrm{n})\end{array}$ & $\begin{array}{c}2,689 \\
(66.12)\end{array}$ & $\begin{array}{c}1,924 \\
(47.31)\end{array}$ & $\begin{array}{c}2,689 \\
(67.31)\end{array}$ & $\begin{array}{c}1,699 \\
(42.53)\end{array}$ & & \\
\hline & Informed, percentage (n) & $\begin{array}{c}1,376 \\
(33.83)\end{array}$ & $\begin{array}{c}2,143 \\
(52.69)\end{array}$ & $\begin{array}{c}1,306 \\
(32.69)\end{array}$ & $\begin{array}{c}2,296 \\
(57.47)\end{array}$ & $.25(.06)^{\mathrm{c}}$ & 1.28 \\
\hline \multicolumn{2}{|c|}{ IDM: continuous (0-48), mean $(S D)$} & $\begin{array}{c}18.95 \\
(11.45)\end{array}$ & $\begin{array}{c}24.28 \\
(11.82)\end{array}$ & $\begin{array}{c}18.69 \\
(11.21)\end{array}$ & $\begin{array}{c}25.85 \\
(12.30)\end{array}$ & $1.72(.27)^{c}$ & 0.007 \\
\hline \multicolumn{2}{|c|}{ Decisional conflict (1-7), mean $(S D)$} & $\begin{array}{c}4.33 \\
(1.74)\end{array}$ & $\begin{array}{c}5.17 \\
(1.45)\end{array}$ & $\begin{array}{c}4.33 \\
(1.75)\end{array}$ & $\begin{array}{c}5.38 \\
(1.36)\end{array}$ & $.21(.04)^{\mathrm{c}}$ & 0.008 \\
\hline \multicolumn{2}{|c|}{ Intention (1-7), mean $(S D)$} & $\begin{array}{c}5.35 \\
(1.70)\end{array}$ & $\begin{array}{c}5.42 \\
(1.97)\end{array}$ & $\begin{array}{c}5.35 \\
(1.69)\end{array}$ & $\begin{array}{c}5.59 \\
(1.87)\end{array}$ & $.18(.03)^{\mathrm{c}}$ & 0.006 \\
\hline \multicolumn{2}{|c|}{ Attitude (1-7), mean (SD) } & $\begin{array}{c}5.19 \\
(1.46)\end{array}$ & $\begin{array}{c}5.22 \\
(1.57)\end{array}$ & $\begin{array}{c}5.18 \\
(1.45)\end{array}$ & $\begin{array}{c}5.37 \\
(1.51)\end{array}$ & $.15(.03)^{c}$ & 0.006 \\
\hline \multicolumn{2}{|c|}{ Beliefs (1-7), mean $(S D)$} & $\begin{array}{l}4.21 \\
(.72)\end{array}$ & $\begin{array}{l}4.37 \\
(.80)\end{array}$ & $\begin{array}{l}4.19 \\
(.73)\end{array}$ & $\begin{array}{l}4.47 \\
(.81)\end{array}$ & $.12(.02)^{c}$ & 0.010 \\
\hline \multicolumn{2}{|c|}{ Risk perception not vaccinated (1-7), mean $(S D)$} & $\begin{array}{l}3.73 \\
(.98)\end{array}$ & $\begin{array}{c}3.70 \\
(1.05)\end{array}$ & $\begin{array}{c}3.74 \\
(0.98)\end{array}$ & $\begin{array}{c}3.77 \\
(1.08)\end{array}$ & $.06(.02)^{\mathrm{d}}$ & 0.001 \\
\hline \multicolumn{2}{|c|}{$\begin{array}{l}\text { Risk perception vaccinated } \\
(1-7), \text { mean }(S D)\end{array}$} & $\begin{array}{c}2.76 \\
(1.06)\end{array}$ & $\begin{array}{c}2.74 \\
(1.08)\end{array}$ & $\begin{array}{c}2.77 \\
(1.07)\end{array}$ & $\begin{array}{c}2.64 \\
(1.10)\end{array}$ & $-.11(.03)^{c}$ & 0.004 \\
\hline \multicolumn{2}{|c|}{ Anticipated regret (1-5), mean $(S D)$} & $\begin{array}{c}3.68 \\
(1.27)\end{array}$ & $\begin{array}{c}3.50 \\
(1.33)\end{array}$ & $\begin{array}{c}3.71 \\
(1.25)\end{array}$ & $\begin{array}{c}3.59 \\
(1.31)\end{array}$ & $.07(.03)^{\mathrm{d}}$ & 0.001 \\
\hline \multicolumn{2}{|c|}{ Subjective norm ( -20 to 20$)$, mean $(S D)$} & $\begin{array}{c}5.92 \\
(7.90)\end{array}$ & $\begin{array}{c}6.46 \\
(9.46)\end{array}$ & $\begin{array}{c}5.88 \\
(7.81)\end{array}$ & $\begin{array}{c}7.25 \\
(9.20)\end{array}$ & $.82(.20)^{\mathrm{c}}$ & 0.004 \\
\hline \multicolumn{2}{|c|}{ Habit (1-7), mean $(S D)$} & $\begin{array}{c}4.26 \\
(1.79)\end{array}$ & $\begin{array}{c}4.36 \\
(1.82)\end{array}$ & $\begin{array}{c}4.28 \\
(1.78)\end{array}$ & $\begin{array}{c}4.51 \\
(1.83)\end{array}$ & $.14(.04)^{c}$ & 0.004 \\
\hline \multicolumn{2}{|c|}{ Relative effectiveness (1-10), mean $(S D)$} & $\begin{array}{l}-2.01 \\
(2.24)\end{array}$ & $\begin{array}{l}-1.84 \\
(2.36)\end{array}$ & $\begin{array}{l}-1.97 \\
(2.22)\end{array}$ & $\begin{array}{l}-1.35 \\
(2.27)\end{array}$ & $.46(.07)^{\mathrm{c}}$ & 0.015 \\
\hline \multicolumn{2}{|c|}{ Self-efficacy (1-7), mean $(S D)$} & $\begin{array}{l}6.24 \\
(.76)\end{array}$ & $\begin{array}{l}6.24 \\
(.78)\end{array}$ & $\begin{array}{l}6.27 \\
(.73)\end{array}$ & $\begin{array}{l}6.29 \\
(.75)\end{array}$ & $.04(.02)^{\mathrm{e}}$ & 0.001 \\
\hline \multicolumn{2}{|c|}{ Knowledge ( -8 to 8$)$, mean $(S D)$} & $\begin{array}{c}4.42 \\
(2.16)\end{array}$ & $\begin{array}{c}5.41 \\
(2.09)\end{array}$ & $\begin{array}{c}4.40 \\
(2.14)\end{array}$ & $\begin{array}{c}5.75 \\
(2.09)\end{array}$ & $.35(.05)^{\mathrm{c}}$ & 0.009 \\
\hline
\end{tabular}

${ }^{a}$ Human papillomavirus (HPV) vaccination uptake was not assessed at baseline.

${ }^{\mathrm{b}} p=.60 . \quad{ }^{c} p \leq .001$, thus significant $(p<.003$; Bonferroni: $0.05 / 14$ factors $)$.

${ }^{\mathrm{d}} p=.01$. $\quad{ }^{\mathrm{e}} p=.03$. 


\section{Moderation of intervention effects}

Regarding socio-demographics, no significant interaction effects on any of the outcome measures were found for country of birth $(p \geq .08)$ or religion $(p \geq .08)$. For educational level, we found an interaction effect with condition on relative effectiveness $(\beta=.59, p$ $<.001)$ : the intervention had more positive effects on relative effectiveness for those with high education compared with those low in education. There was no significant interaction between condition and sample on any of the outcome measures ( $p \geq .04)$. For the interaction effects between intention at baseline and the outcome measures, See Table 5.4. If an OR is higher than one, this means that the higher the score on a factor, the higher the outcome of IDM or the higher the chance of the daughter being vaccinated. If an OR is less than one, this means that the higher the score on a factor, the lower outcome of IDM or the lower the chance of the daughter being vaccinated. In the first 2 columns, the reference category is those with a negative intention. For a comparison between those in doubt (reference category) and a positive attitude, see the third column. Significant interaction effects between intention at baseline and condition were found on intention, attitude, decisional conflict, subjective norm, and relative effectiveness. For mothers who had a negative intention, the intervention had more positive effects on intention and relative effectiveness compared with mothers who were doubting $(\beta=.26, p=.002 ; \beta=.39, p=.001$, respectively) or had a positive intention $(\beta=.40, p<.001 ; \beta=.53, p<.001$, respectively). In addition, for mothers with a negative intention, the intervention had a more positive effect on attitude ( $\beta=.21, p=.001)$ and on subjective norms $(\beta=1.64, p<.001)$ compared with mothers with a positive intention. For mothers who were doubting, the intervention had more positive effects on decisional conflict compared with mothers who had a negative intention $(\beta=.26$, $p=.001)$. No differences on intervention outcomes were found between mothers who were doubting and mothers who had a positive intention $(p \geq .004)$.

\section{Subjective program evaluation and objective program use}

Mothers in the intervention condition evaluated the website with a $7.6(S D=1.36)$ and the virtual assistants with a $7.4(S D=1.53)$. According to the computer logs, $2,509(62.80 \%)$ of the 3,995 (100.00\%) invited mothers logged on to the website. Of these, 1,835 (73.14\%) visited the website once, 498 (19.84\%) visited twice, and $176(7.02 \%)$ more than twice. On average, mothers spent $22 \mathrm{~min}$ on the website $(S D=13 \mathrm{~min})$. 
Table 5.4 Moderation effects of intention subgroups on the outcome measures according to the intention-to-treat analyses $(\mathrm{N}=8,062)$.

\begin{tabular}{|c|c|c|c|c|c|c|c|}
\hline \multirow[b]{2}{*}{ Outcome } & & \multicolumn{2}{|c|}{ Negative-in doubt } & \multicolumn{2}{|c|}{ Negative-positive } & \multicolumn{2}{|c|}{ In doubt-positive } \\
\hline & & $\begin{array}{c}\text { Beta } \\
\text { (standard } \\
\text { error) }\end{array}$ & $p$ value & $\begin{array}{c}\text { Beta } \\
\text { (standard } \\
\text { error) }\end{array}$ & $p$ value & $\begin{array}{c}\text { Beta } \\
\text { (standard } \\
\text { error) }\end{array}$ & $p$ value \\
\hline \multicolumn{8}{|l|}{ Primary outcome } \\
\hline \multirow[t]{2}{*}{$\begin{array}{l}\mathrm{HPV}^{\mathrm{a}} \text { vaccination } \\
\text { uptake }\end{array}$} & $\begin{array}{l}\text { Has received no HPV } \\
\text { injection (reference) }\end{array}$ & & & & & & \\
\hline & $\begin{array}{l}\text { Has received one or } \\
\text { two HPV injections }\end{array}$ & $-.21(.13)$ & .22 & $.01(.18)$ & .97 & $.22(.18)$ & .24 \\
\hline \multicolumn{8}{|l|}{ Secondary outcomes } \\
\hline \multirow[t]{2}{*}{$\mathrm{IDM}^{\mathrm{b}}$ : dichotomous } & $\begin{array}{l}\text { Not informed } \\
\text { (reference) }\end{array}$ & & & & & & \\
\hline & Informed & $.18(.15)$ & .22 & $.09(.15)$ & .56 & $-.10(.14)$ & .50 \\
\hline IDM: continuous $(0-48)$ & & $.92(.69)$ & .18 & $1.03(.65)$ & .12 & $.11(.65)$ & .87 \\
\hline Decisional conflict (1-7) & & $.26(.08)$ & $.001^{\mathrm{c}}$ & $.04(.07)$ & .57 & $.22(.08)$ & .004 \\
\hline Intention (1-7) & & $-.26(.08)$ & $.002^{\mathrm{c}}$ & $-.40(.08)$ & $<.001^{\mathrm{c}}$ & $-.14(.07)$ & .03 \\
\hline Attitude (1-7) & & $-.17(.06)$ & .009 & $-.21(.06)$ & $.001^{\mathrm{c}}$ & $-.04(.06)$ & .51 \\
\hline Beliefs (1-7) & & $-.04(.04)$ & .36 & $-.02(.04)$ & .68 & $.02(.04)$ & .67 \\
\hline Risk perception not vacci & nated (1-7) & $.04(.07)$ & .51 & $.09(.07)$ & .18 & $.05(.06)$ & .41 \\
\hline $\begin{array}{l}\text { Risk perception vaccinate } \\
(1-7)\end{array}$ & & $.04(.06)$ & .51 & $-.06(.07)$ & .38 & $-.10(.07)$ & .17 \\
\hline Anticipated regret (1-5) & & $-.03(.07)$ & .70 & $.02(.07)$ & .81 & $.04(.06)$ & .46 \\
\hline Subjective norm $(-10$ to 1 & & $-1.18(.47)$ & .01 & $-1.64(.43)$ & $<.001^{\mathrm{c}}$ & $-.46(.37)$ & .22 \\
\hline Habit (1-7) & & $.08(.08)$ & .34 & $.06(.08)$ & .45 & $-.01(.07)$ & .87 \\
\hline Relative effectiveness (1-1 & & $-.39(.12)$ & $.001^{\mathrm{c}}$ & $-.53(.12)$ & $<.001^{\mathrm{c}}$ & $-.14(.11)$ & .21 \\
\hline Self-efficacy (1-7) & & $-.00(.05)$ & .97 & $.03(.04)$ & .45 & $.03(.05)$ & .50 \\
\hline Knowledge ( -8 to 8$)$ & & $-.01(.13)$ & .92 & $-.13(.12)$ & .29 & $-.11(.12)$ & .34 \\
\hline
\end{tabular}

${ }^{\mathrm{a}} \mathrm{HPV}$ : human papillomavirus.

${ }^{\mathrm{b}}$ IDM: informed decision making.

${ }^{c} p<.003$, thus significant (Bonferroni: 0.05/14 factors).

\section{DISCUSSION}

\section{Principal findings}

This study investigated the effectiveness of a Web-based tailored intervention with virtual assistants promoting HPV vaccination acceptability among mothers of invited girls. As hypothesized, positive intervention effects were found with respect to the social cognitive determinants of the mothers' decision making about the vaccination (e.g., HPV vaccination- 
related intention, attitude, and outcome beliefs), levels of IDM, and levels of decisional conflict. The positive effect of tailored education on HPV vaccination intention was also found by Gerend et al. (2013) among young women. However, they did not assess other determinants of HPV vaccination acceptability (next to intention) nor did they measure levels of IDM, levels of decisional conflict, or actual HPV vaccination uptake.

The findings described above suggest that this intervention has potential in promoting HPV vaccination acceptability and IDM. This is important, given the currently moderate HPV vaccination uptake and the fact that large proportions of the mothers do not actively acquire and process information about the pros and cons of this HPV vaccination and that many feel ambivalent about the decision (Van Keulen et al., 2013a; Van Keulen et al., 2013b). Less informed decisions are decisions constituted in rather instable beliefs that are susceptible to counterarguments. Nowadays, counterarguments are all around on the Internet and Web-based social media (Kata, 2012). Because the intervention initiated active processing of verifiable information about the risks and effectiveness of the HPV vaccination, it inoculates mothers with arguments that become accessible at the moment they are confronted with (new) information that might challenge their initial positive attitudes and intentions (McGuire, 1964; Paulussen et al., 2006).

No effects were found on mothers' perceived risk of their daughter getting cervical cancer without the HPV vaccination, anticipated regret in case their daughter would get cervical cancer later in life, and self-efficacy. As for risk perception, baseline scores indicated that the mothers overestimated the probability of contracting cervical cancer to a great extent when taken into account the actual population incidence (Integraal Kankercentrum Nederland, 2016). Because the intervention presented mothers this actual low probability of attracting cervical cancer, it seems unlikely that their perceived risk was brought to higher levels. The lack of effect on anticipated regret might be explained by the fact that we removed the intervention component specifically targeting anticipated regret. This was removed because our pilot studies and focus groups revealed that resistance was evoked by asking mothers how much regret they would have if they did not vaccinate their daughter against HPV and their daughter developed cervical cancer later in life. Also, emphasizing the impact of cervical cancer might be fear-arousing, which, in turn, may have been detrimental for exploring and processing other information provided by the program (Ruiter, Abraham, \& Kok, 2001). Finally, it appeared that we encountered a ceiling effect for self-efficacy as the scores at baseline among both groups were above 6 on a 7 -point scale.

No effects of the tailored intervention were found on HPV vaccination uptake. This is contrary to both our expectations and to what has been found by others; Hopfer (2012) found that among female college students, HPV vaccination uptake doubled after they were 
exposed to a tailored video compared with controls ( $22 \%$ vs $12 \%$, respectively). However, in their study, vaccination rates were quite low in the control condition (12\%), leaving much room for improvement. In our study, however, the uptake rates were high in both conditions (intervention: $\mathrm{n}=3,995 ; 73.17 \%$ and control: $\mathrm{n}=4,067 ; 72.71 \%$ ) especially when compared with the national Dutch uptake ( $n=59,866 ; 60.98 \%$ ) (Van Lier et al., 2016). This may explain why we, as opposed to Hopfer, did not find an effect on HPV vaccination uptake. After all, we did find a larger increase in intention among mothers in the intervention compared with the control condition, and according to theory (Fishbein \& Ajzen, 2010) and empirical findings (Hofman et al., 2014), intention is an important predictor of (HPV vaccination) behavior.

Mothers evaluated the intervention as positive, specified by the high subjective evaluation of both the website ( 7.6 on a 10 -point scale) and the virtual assistants that were used to deliver the tailored feedback (7.4 on a 10-point scale). Objective program use was also high, with $62.80 \%(n=2,509)$ of the invited mothers having visited the website. In addition, on average, they spent quite some time on the website $(22 \mathrm{~min})$. Taken together, the intervention has potential for broad national dissemination and implementation.

Furthermore, subgroup analysis with 3 intention groups (i.e., negative, in doubt, and positive) showed that the intervention had the most positive effects on decisional conflict for mothers who were doubting. For mothers with a negative intention, the most positive effects were found on intention, attitude, and subjective norms. This is promising, as for a population-wide program it is relevant to guide those in doubt toward making an informed choice without decisional conflict and to persuade those having negative intentions toward vaccination. Fortunately, we did not find any adverse effects of the intervention in mothers with a negative intention, such as a further decrease in their intention or attitude.

\section{Methodological considerations}

There are two methodological considerations. First, with large sample sizes, as in this study, even small effects can become statistically significant (Lin, Lucas, \& Shmueli, 2013). However, the positive intervention effects were consistently found on almost all outcomes. In addition, the large sample provided us with sufficient data for conducting analyses on subsamples (i.e., based on socio-demographics, sample, and three intention groups) while maintaining sufficient levels of power (Lin, Lucas, \& Shmueli, 2013). The effect sizes that we found are in line with other Web-based interventions targeting health behavior outcomes (Webb et al., 2010). We believe these, even small effects, are of relevance in public health as they become substantial at the population level. In addition, the intervention was of help for 
those in doubt and did not have any detrimental effect. We therefore find this Web-based tailored program is a substantial step forward in improving both research and practice in the context of the promotion of HPV vaccination acceptability.

Second, we used a scale comprising two items of the Self-Report Habit Index for measuring habit (Verplanken \& Orbell, 2003). This accounted for the extent to which getting the HPV vaccination was something mothers did (1) naturally and (2) without thinking. The positive intervention effect on the composite measure might indicate that the intervention induced perceptions about the HPV vaccination as something you take for granted, without thinking. The latter is unwanted, considering the aim of initiating active processing of information about the vaccination. Fortunately, secondary analysis separating the two items showed that there was a positive effect of the intervention on the first item (i.e., "naturally") but not on the second (i.e., "without thinking"). In retrospect, the label "habit" attributed to the two-item operationalization appears misleading, though the intervention strengthened the mothers' belief about getting the HPV vaccination as something natural.

\section{Strengths and limitations}

Important strengths of this study are the randomized controlled design, adequate sample sizes, and the reliable objective assessment of HPV vaccination uptake. Furthermore, the systematic, stepwise development of the intervention was a notable strength, as well as the mothers' positive subjective evaluation of the intervention and the objectively assessed high level of program use.

Some limitations of this study should be considered as well. First, the study was subject to a considerable amount of attrition. Unfortunately, attrition is quite common in studies on Web-based interventions (Kohl, Crutzen, \& de Vries, 2013). Dropout analysis showed that dropout was selective. For instance, there were higher dropout rates in the intervention condition, which has also been reported for other (tailored) interventions (Oenema et al., 2008; Oenema, Tan, \& Brug, 2005; Spittaels, de Bourdeaughuij, \& Vandelanotte, 2007). In this study, we handled the missing data and selective dropout by using multiple imputation (Twisk, 2006). Results from the complete case analyses only slightly differed from those from the ITT analyses. Thus, it seems unlikely that the observed effects are spurious or due to selective dropout. Second, caution is needed when generalizing the results of this study to the general population (i.e., Dutch mothers of girls aged 12 years) because we were unable to check the sample's representativeness. However, we did not find any differences in effectiveness of the intervention in specific subgroups of participants, as indicated by the conducted moderation analyses with socio-demographics. 


\section{Conclusions and recommendations}

The study findings suggest that this Web-based tailored intervention has the potential to improve both HPV vaccination acceptability and IDM, and decrease decisional conflict among mothers of invited girls. Therefore, we recommend nationwide dissemination and implementation. Furthermore, we recommend developing (tailored) interventions targeting the daughter and the mother's partner, as these have appeared to be important social referents (Van Keulen et al., 2013a; Van Keulen et al., 2013b). Research has indicated that tailoring the intervention could have beneficial effects for girls, as they also expressed their need for interactive and personal information about the HPV vaccination (Van Keulen et al., 2010). This still needs to be investigated for the partners. In addition, boys may also become a relevant target group. In other countries, such as Australia, boys are already included in the national immunization program. This may contribute to the achievement of herd immunity and to a reduction of the global burden of a variety of HPV-related cancers in women and men (Braakhuis, Leemans, \& Visser, 2014; Georgousakis et al., 2012; Wakeham \& Kavanagh, 2014).

\section{Acknowledgments}

We would like to thank Petra Oomen (RIVM) and the panels (Veldkamp BV, Intromart GFK, and NGO Flycatcher) for their help with data collection. ZonMw (The Netherlands Organization for Health Research and Development) and NWO (Netherlands Organization for Scientific Research) support this study (grant-number: 50-51515-98-259).

\section{Conflicts of Interest}

None declared. 



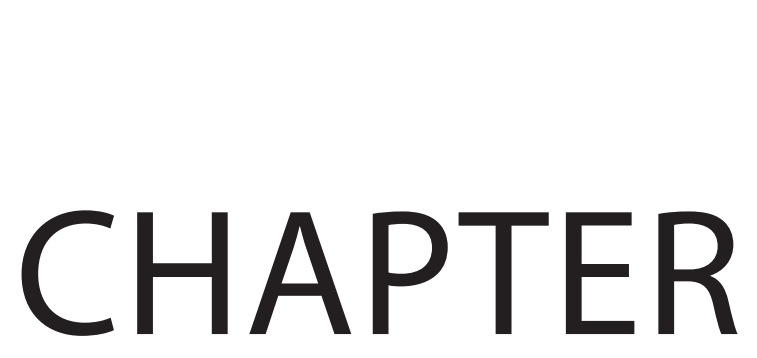

General Discussion

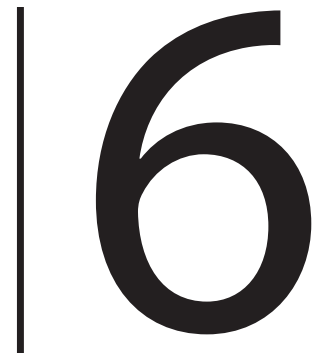





\section{GENERAL DISCUSSION}

The studies that have been described in this $\mathrm{PhD}$ thesis informed the development and evaluation of an interactive, Web-based, tailored intervention promoting HPV vaccination acceptability among mothers of invited girls. The present dissertation is divided into three parts: (1) the needs assessment (Chapter 2), (2) intervention development (Chapters 3 and 4), and (3) intervention evaluation (Chapter 5). This chapter starts with an overview of the main findings and a critical reflection on these findings. It then outlines the limitations and strengths of the studies. Finally, implications for future research and practice will be discussed.

\section{MAIN FINDINGS}

\section{PART I: The Needs Assessment}

The first part of this thesis was about the stage of the needs assessment. Previous research in the Netherlands has shown that mothers play the most important role in the decision about their daughters' HPV vaccination (Van Keulen et al., 2010). Therefore, mothers of girls to-be-invited were selected as the primary target group for the intervention to be developed. We aimed to gain insight into the determinants of the mothers' HPV vaccination acceptability. Earlier studies on determinants of HPV vaccination acceptability were either cross-sectional (e.g., Van Keulen et al., 2013a), or focused on HPV vaccination acceptability before the HPV vaccination was implemented (e.g., Brewer \& Fazekas, 2007). We extended these findings by conducting a longitudinal study that accounted for HPV vaccination uptake after implementation of the national HPV vaccination campaign (Chapter 2). The results showed that intention was the single best predictor of HPV vaccination uptake. Intention, in turn, was best explained by attitude, behavioral beliefs, subjective norms, habit and perceptions about the relative effectiveness of the vaccine. Less important (but still relevant) for the mothers' intention were anticipated regret, risk perception, self-efficacy, and knowledge.

The longitudinal study provides a strong theoretical basis for predicting HPV vaccination uptake and, hence, a robust foundation for intervention development. First, because the results confirm what was found in former cross-sectional studies and thus give support to the application of social cognitive models such as the Reasoned Action Approach (Fishbein \& Ajzen, 2010) for predicting HPV vaccination uptake. Second, the amount of explained variance was high; the psychosocial determinants described above explained $83 \%$ of the mothers' intention, while intention explained $43 \%$ of the variance in uptake. This exceeds the average proportion of explained variances found in meta-analysis of a variety of health- 
related behaviors (Sheeran, 2002). This is also a much higher percentage when compared to the study of Juraskova and colleagues (2012), who found that intention only predicted small proportions of variation in HPV vaccination behavior (9.6\%).

Furthermore, our study showed that intention appeared to be predominantly attitudedriven (Chapter 2). Specifically, the general evaluation of HPV vaccination acceptance was driven by beliefs about (1) the responsibility of the government with regard to the HPV vaccination, (2) the daughters' sexual behavior and age in relation to the need for the HPV vaccination, (3) the safety and effectiveness of the HPV vaccination, and (4) the impact of pharmaceutical industries on national immunization policies. Furthermore, our study showed that certain misperceptions regarding the HPV vaccination exist. For instance, mothers considered the HPV vaccination being less effective in preventing cervical cancer than having a healthy lifestyle (Chapter 2). This has also been found in previous research (Van Keulen et al., 2010). This misperception needs correction, since the available evidence shows us that a healthy lifestyle is not sufficient in preventing cervical cancer whereas HPV vaccination can (International agency for research on cancer, 2005).

\section{PART II: Intervention Development}

The needs assessment provided us with the target group (i.e., mothers) and targets for designing the intervention; these were attitude, behavioral beliefs, subjective norms, habit, relative effectiveness, anticipated regret, risk perception, self-efficacy, and knowledge (Chapter 2). The second part of this $\mathrm{PhD}$ thesis was about the systematical development of an interactive, Web-based, tailored intervention using the Intervention Mapping (IM) protocol (Chapters 3-4) (Bartholomew Eldredge et al., 2016).

\section{Computer-tailoring}

Computer-tailoring was the basic method for change as this suited the need for personalized information about the HPV vaccination as was indicated by the mothers (Van Keulen et al., 2010). Besides, computer-tailored interventions have the potential to enable large scale application at relatively low costs (Neville, O'Hara, \& Milat, 2009) and can therefore have a substantial impact at the population (Noar, Benac, \& Harris, 2007). Moreover, metaanalyses have shown that computer-tailored interventions are more effective than generic interventions in achieving behavioral health outcomes, for a variety of behaviors and in diverse populations (Krebs et al., 2010; Lustria et al., 2013). Tailoring has only recently been applied to HPV vaccination (Bennett et al., 2013; Gerend et al., 2013; Grandahl et al., 2016; Hopfer, 2012). To our knowledge, only one of the existing tailored interventions 
was computer-tailored and this intervention turned out to be ineffective in promoting HPV vaccination acceptance (Bennett et al., 2015). In the Netherlands, computer-tailored interventions promoting (HPV) vaccination did not exist yet at the start of this $\mathrm{PhD}$ thesis.

Throughout the intervention, tailoring was used in three different ways. First, it was used to tailor the feedback on participants' answers to statements and questions about specific aspects of the HPV vaccination (e.g., by correcting misperceptions regarding the HPV vaccination). Second, computer-tailoring was used to provide mothers the opportunity to weigh their personal values regarding the HPV vaccination (e.g., with a decisional balance). Finally, computer-tailoring was used for guiding mothers through the website (e.g., by highlighting parts of the intervention which the mother had not seen yet). Thus, not only did we tailor the content of the intervention to the mothers' personal interest, but it was also used to guide the mothers' personal pathway through the intervention. The latter is likely to have improved the usability of the intervention. The intervention accounted for tailoring on a variety of subjects. For example, not only did we tailor on perceived barriers (e.g., beliefs about adverse effects), like Gerend and colleagues (2013) did, but also on other beliefs (such as beliefs about the daughters' sexual behavior and age in relation to the need for the HPV vaccination), attitude, subjective norms, habit, relative effectiveness, anticipated regret, risk perception, self-efficacy, and knowledge.

\section{Virtual assistants}

Two virtual assistants (i.e., a mother-like and doctor-like assistant) delivered tailored feedback throughout the intervention. The added value of using the virtual assistants is that it matched the mothers' preferences for more interactive personalized feedback (Van Keulen et al., 2010). In addition, using virtual assistants improves information recall (Beun, de Vos, \& Witteman, 2003), transfer of learning (Atkinson, 2002), amount of learning (Baylor \& Kim, 2004), self-efficacy expectations, literacy and behavioral change (Blanson Henkemans et al., 2009; Blanson Henkemans et al., 2008; Jin, 2010).

The use of virtual assistants in interventions promoting HPV vaccination acceptance seems promising since results from the focus groups (Chapter 4) and the subjective program evaluation (Chapter 5) showed that mothers appreciated them very well. But, we still consider the use of virtual assistants to be complex, especially in Web-based interventions in which both spoken and written feedback/information are provided. In one of the first prototypes of our intervention, for instance, the spoken and written information were presented at the same time (conveyed by the virtual assistant and website, respectively). However, mothers indicated that they experienced difficulties with reading and listening to 
the virtual assistant at the same time. Hence, we created a new prototype of the website, in which the written feedback showed up after the virtual assistant had completed the spoken feedback.

\section{Advantages and disadvantages of systematic development}

The Intervention Mapping (IM) protocol was used to ensure a solid theoretical and empirical foundation for the intervention (cf. Bartholomew Eldredge et al., 2016) (Chapters 3-4). The surplus value of this systematical development is that it contributes to the likelihood of creating an intervention that is both effective and appreciated by the target group. Also, using IM has made the development process explicit and transparent, providing a road map of the decision-making process and its main outcomes.

When developing an intervention using the IM protocol, one does have to take into account that this is a complex and time-consuming process (Côté et al., 2008; Heinen et al., 2006; Van Kesteren et al., 2006). However, we believe that the experience we gained may improve efficacy of the process and make it less time-consuming in future intervention development. Other researchers can profit from this experience when developing similar interventions for a different population. Furthermore, using IM requires researches to have extensive and documented expertise on research from various fields, such as the health behavior to be changed (e.g., HPV vaccination acceptance), strategies that are used in the intervention (e.g., behavior change methods) and so forth. However, expertise from multiple fields is necessary to develop an effective intervention (Bartholomew Eldredge et al., 2016). Those wishing to use the IM protocol in future should ensure that relevant expertise is available prior to embarking on this process.

\section{User-centered design}

According to IM, it is imperative that members of the target group are involved in the development of the intervention (Bartholomew Eldredge et al., 2016). However, currently, in many eHealth interventions, the design of the intervention is based on assumptions that are not validated with input from end-users. In fact, the importance of formative research and pretesting of materials is often being overlooked. The resulting intervention may therefore lack key features, and subsequent evaluations of the effectiveness of the interventions may be compromised (Cafazzo et al., 2009). Therefore, as described in Chapter 4, we applied a user-centered design by extensively involving mothers in the intervention development from the beginning to the end (Mao et al., 2005). We adapted the entire intervention to the requirements and preferences of the mothers. Not only did we fine-tune the content of the intervention to the mothers' preferences, but also the design of the website was chosen by the mothers. 
We applied a user-centered design by conducting focus groups, online pretests, and experimental pretests. In these, we gathered feedback on different versions (static and interactive) or (parts of the) intervention. For instance, as a result of the focus groups, we created an adapted version of the website in which we aimed to maximize exposure to various components of the intervention by using the virtual assistant to give advice about the components to visit next, based on what the mother had already visited. Also, the suggested component was highlighted to improve usability. The online pretests were to ensure that it worked adequately on various devices (e.g., on different types of tablets). Besides, three experimental pretests assessing outcomes were conducted. These experimental pretests enabled us to draw reliable conclusions about the effectiveness of the selected applications in influencing the predetermined objectives and served as a safeguard against selecting inadequate intervention strategies (Whittingham et al., 2008a; Whittingham et al., 2008b).

One of these experimental pretests tested strategies for affecting mothers' perceived daughters' susceptibility towards HPV-related risk (Chapter 3). Two types of risk information (i.e., statistical and narrative risk information) were pretested as research remains to be mixed on the surplus value of one type over the other in influencing perceived susceptibility (e.g., De Wit, Das, \& Vet, 2008 versus Mevissen et al., 2009). The results showed that statistical risk information seemed most effective in increasing mothers' perceived susceptibility towards HPV of their daughters. Therefore, we decided to use statistical risk information for the intervention component communicating about HPV-related risks. Furthermore, we found that, in general, mothers tend to underestimate the probability of their daughter getting infected with HPV, while they tend to overestimate the chance of their daughter attracting cervical cancer (Chapter 3). The tendency to overestimate small frequencies and to underestimate larger ones, is well known (Fischhoff, Bostrom, \& Quadrell, 1993; Lichtenstein et al., 1978). Therefore, in the intervention, the information was tailored to the mothers' risk perception and corrected misperceptions (when present). This provided mothers with a more realistic knowledge base for initiating their processes of decision making.

In a second online experimental pretest, we explored the best way to communicate about social norms; by providing negatively (i.e., discourage undesired behavior) versus positively (i.e., encourage desired behavior) framed descriptive and/or injunctive norms (Chapter 4). We found no indication for using one type of framing norms over the other. As negatively framed norms were expected to be more difficult to process since they include negations (Gilbert, 1991), we decided to just include positively framed norms. The descriptive norm was included by communicating about the national HPV vaccination uptake rate. And, within the component 'talking about the HPV vaccination', mothers were taught how to 
deal with a potential contrasting injunctive norm (i.e., either positive or negative attitude and intention) of their daughter, partner, general practitioner, other family members, and/ or other parents.

In the final experimental pretest, we investigated the effects of acknowledging versus ignoring uncertainty about potential long-term effects of the HPV vaccination (Chapter 4). Results showed that compared to mothers who were exposed to information ignoring uncertainty, mothers who were exposed to information acknowledging uncertainty experienced more decisional conflict, were more ambivalent about their decision, and had a less positive attitude and intention towards HPV vaccination. Although these findings implicated not to communicate about long-term uncertainties, we chose to do so in the intervention. The main reason for this was that not communicating about long term uncertainties brings along the risk of mothers searching information about this elsewhere. This can be quite dangerous as many rumors about potential long-term effects, for which no prove exists, can be found on the internet and online social media (Kata, 2012). Reading these (false) rumors without any refutation being offered aside (Allen, 1991), could have more detrimental effects on HPV vaccination acceptability than when we ourselves provide the (correct) information. The latter enables mothers to make an informed decision, which was one of the desired outcomes of the intervention. Having made an informed decision, in turn, inoculates mothers with arguments that become accessible in case they are confronted with (new) information that might challenge their initial positive intentions (i.e., psychological inoculation) (McGuire, 1964; Paulussen et al., 2006). Moreover, mothers themselves expressed a need for full disclosure, especially when uncertainties were ignored, which was also found in a previous study (Van Keulen et al., 2010).

Although experimental pretests such as the three described above provide valuable information for intervention development, it should be noted that pretesting all components of an intervention is not feasible. After all, such experiments require a great amount of time and frequently financial constraints are present (Whittingham et al., 2008b). Alternatively, insight into active ingredients of other interventions may contribute to implementing effective strategies without pretesting them beforehand. In order to identify active ingredients, it is important that interventions are well described (Bartholomew \& Mullen, 2009; Kok \& Mesters, 2011). Chapter 4 provides such a detailed, systematic description of an intervention. 


\section{PART III: INTERVENTION EVALUATION}

In the third part of this thesis, we conducted both an effect and process evaluation of the interactive, Web-based, tailored intervention (Chapter 5). This enabled us to determine whether and how the intervention worked, if the intervention was appropriate for affecting the target population, and whether there were any problems with its implementation.

\section{Effect Evaluation}

An RCT was conducted in which exposure to the tailored intervention (i.e., the experimental group) was compared to the existing communication about the HPV vaccination (i.e., the control group) (Chapter 5) in two differential samples (taken from internet panels and from Praeventis). The novelty of this effect evaluation is that it had a broader scope of outcomes compared to other studies (see Fu et al., 2014 for a review), such as the one conducted by Gerend and colleagues (2013). Our study accounted for HPV vaccination intention, determinants of intention that had proved to be of relevance, levels of informed decision-making, levels of decisional conflict, and actual HPV vaccination uptake. The fact that we objectively assessed the HPV vaccination uptake is quite new. According to several reviews (Fu et al., 2014; Priest \& Knowlden, 2015), most others just relied on self-reports which might be subject to bias.

Results from the RCT showed that positive intervention effects were found on almost all determinants of HPV vaccination uptake (i.e., intention, attitude, beliefs, risk perception when vaccinated, subjective norms, relative effectiveness, and knowledge), informeddecision making and decisional conflict (Chapter 5). This is important given the currently moderate HPV vaccination uptake and the fact that large proportions of the mothers do not actively acquire and process information about the pros and cons of this HPV vaccination, while many feel ambivalent about the decision (Van Keulen et al., 2010). Less informed decisions are constituted in rather instable beliefs which are susceptible to counterarguments. Nowadays, counterarguments are all around on the internet and online social media (Kata, 2012). Such anti-vaccination lobbying has been shown to increase perceived risk of vaccinations and to decrease intentions to get vaccinated (Betsch, Renkewitz, \& Ulshöfer, 2010). This stresses the importance of interventions initiating active processing of verifiable information about the risks and effectiveness of the HPV vaccination, so that it inoculates mothers with arguments that become accessible at the moment they are confronted with (new) information that might challenge their initial positive attitudes and intentions (McGuire, 1964; Paulussen et al., 2006). 
At first glance, no effect of the tailored intervention was found on HPV vaccination uptake (Chapter 5). This was contrary to both our expectations and to what has been found elsewhere. For example, Hopfer (2012) found that among female college-aged students, HPV vaccination uptake doubled after they were exposed to a tailored video compared to controls (22\% versus $12 \%$, respectively). In their study, vaccination rates were quite low in the control condition (12\%), leaving much room for improvement. In our study, however, the uptake rates were high in both conditions (73\% in both intervention and control) especially when compared to the national Dutch uptake (46\%) (Van Lier et al., 2018). This may explain why we, as opposed to Hopfer (2012), did not find an effect on HPV vaccination uptake. However, secondary analyses showed that HPV vaccination uptake was positively influenced by the extent to which mothers were exposed to the online tailored intervention (Hofstra, 2017). And, exposure had a positive effect on HPV vaccination intention and informed decision-making. In other words, the more mothers were exposed to the intervention, the more likely they were to make an informed decision, develop a positive intention towards the HPV vaccination and to have their daughter vaccinated against HPV. This has also been found in de field of influenza vaccination among health care workers: the higher the exposure the higher the vaccine uptake (Looijmans-van der Akker et al., 2010).

In order to test the efficacy and the effectiveness of the intervention, two differential samples were used. The first sample consisted of mothers of girls-to-be-invited for the HPV vaccination, drawn from the National Immunization Register. This sample enabled us to assess the effectiveness of the intervention under more natural conditions and thus get a more reliable indication of the effects and response rates to be expected when the intervention has been implemented nationally. The second sample consisted of mothers of 12-year-old girls from internet panels that showed high response rates (76\%) in earlier research on HPV vaccination (Van Keulen et al., 2013a; Van Keulen et al., 2013b). This provided us the means to better control for acceptable levels of participation and fidelity of intervention exposure compared to the naturalistic sample. Therefore, the internet panel sample was a better condition for testing the intervention's efficacy. We assessed potential differential effects of the intervention under more controlled versus more naturalistic conditions, since this is a basis for inferences concerning generalizability of intervention outcomes. Moderation analysis did not show any differences in intervention effects between the two samples (Chapter 5). In other words, there were no differential effects of the intervention under (a) more controlled conditions (i.e., panel sample), and (b) more naturalistic conditions as will be the case when the intervention has become part of the national implementation strategy (i.e., Praeventis sample). Hence, we conclude that intervention outcomes are generalizable and will remain stable once the intervention has been implemented nationally (Ernst \& Pittler, 2006). 
Moderation analyses also showed that there were no differences in intervention effects as far as socio demographics are concerned (i.e., religion, education level, and country of birth) (Chapter 5). As for country of birth, this could be explained by the finding of Alberts and colleagues (2017), who found no major differences in determinants of HPV vaccination uptake in mothers of different ethnic minorities, including native Dutch. Hence, we agree on their conclusion that, there is no critical need for developing separate interventions for different ethnic groups (Alberts et al., 2017). Although there were no differences in intervention effects among these subgroups, it should be noted that a very homogenous sample was reached. An undiversified reach is a known phenomenon within Web-based interventions aimed at health promotion (Kohl, Crutzen, \& De Vries, 2013). In our study, mothers were predominantly born in the Netherlands, highly educated, and likely to have a daughter that was vaccinated against HPV.

Finally, subgroup analysis with three intention groups (i.e., negative, in doubt, positive) showed that the intervention had the most positive effects on decisional conflict for mothers who were doubting, while, the most positive effects on intention, attitude and subjective norms were found in mothers with a negative intention. And fortunately, we did not find any adverse effects of the intervention on mothers with a positive intention, such as a decrease in their intentions or attitudes (Chapter 5). This is promising as for a population wide program it is most important to guide those in doubt towards making an informed decision that decreases their decisional conflict and persuade those with negative intentions towards vaccination. And, not at the expense of negative side effects in those initially positive about the vaccination.

\section{Process Evaluation}

Results from the subjective program evaluation showed that mothers evaluated both the website (7.6 on a 10-point scale) and the virtual assistants positively (7.4 on a 10-point scale) (Chapter 5). We believe this high positive evaluation can be attributed to the process of extensively involving the target group during intervention development. Results from the objective program evaluation showed that objective program use was high, with $62.80 \%$ (n $=2,509$ ) of the invited mothers having visited the website. On average, they spent quite some time on the website (22 minutes; Chapter 5). This is longer than the mean duration that Brouwer and colleagues (2011) found in their review of other Internet-delivered interactive healthy lifestyle interventions, which varied from less than 10 minutes to 10-20 minutes.

As mentioned before, we found that the amount of exposure to the intervention had positive effects on intervention outcomes. Mothers who had visited more pages of the intervention changed to higher levels of informed decision-making and HPV vaccination intention, and were more likely to have vaccinated their daughter against HPV (Hofstra, 2017). This 
is in line with other studies that have indicated dose-response effect in internet-delivered interventions (Brouwer et al., 2011; Christensen, Griffiths \& Korten, 2002; Danaher et al., 2006).

\section{STRENGTHS AND LIMITATIONS}

In this section, we will discuss the strengths and limitations for each part of this thesis (i.e., for the needs assessment, the intervention development, and the intervention evaluation).

\section{PART I: The Needs assessment}

Our sample overrepresented mothers born in the Netherlands, mothers with a high educational level and mothers having a daughter being vaccinated against HPV (Chapter 2). Although this will limit generalization of mean-scores and percentages found in this sample, it will hardly have obscured the tested associations as these are less sensitive to sample selection. But, the needs assessment also had important strengths. First, the needs assessment ensured us that we targeted the group that was most important when it comes to decision-making about the HPV vaccination (i.e., mothers) (Van Keulen et al., 2013a; Van Keulen et al., 2010). Second, the longitudinal design provided a strong theoretical basis for predicting HPV vaccination uptake and, hence, a robust foundation for intervention development (Chapter 2). Finally, the needs assessment revealed insight into the preferred mode of delivering information about the HPV vaccination.

\section{PART II: Intervention Development}

As mentioned before, IM is a time-consuming process. Sometimes, we considered it to be quite a challenge to adhere to the time schedules we had to take into account. But it was worth the challenge. First, because we considered the user-centered design and the accompanying preliminary research to be a notable strength. By means of focus groups, online pretests and experimental pretests we ensured that the intervention matched the preferences of the mothers (i.e., a Web-based, tailored and interactive intervention) and that no inadequate strategies and materials were included in the intervention. Another strength of the intervention was the use of computer-tailoring. This enables dissemination of the intervention among large numbers at relatively low costs (Neville O'Hara, \& Milat, 2009) and can therefore have substantial impact at the population level (Noar, Benac, \& Harris, 2007). In the Netherlands, the percentage of Internet availability and usage is high; $86 \%$ of individuals are daily users and $97 \%$ of households has access to the Internet (Eurostat, 2017). Finally, an important strength of intervention development was the use of virtual assistants to provide the (computer-) tailored feedback, which is highly innovative in the field of (HPV) vaccination. 
A limitation of the intervention is that the effect sizes were small (Chapter 5). This is in line with other Web-based interventions targeting health behavior outcomes (Webb et al., 2010). Still, we believe these even small effects are of relevance in public health as they become substantial at the population level. After all, in the Netherlands, approximately 100,000 girls are invited for the HPV vaccination on a yearly base. An explanation for the small effect sizes in our study is that mothers were unlikely to have been exposed to all of the behavior change components that were included in the intervention. Support for this notion comes from the finding of the positive effect of exposure to the intervention (and thus increased exposure to more intervention content) on several outcome measures, including actual uptake (Hofstra, 2017).

\section{PART III: Intervention Evaluation}

A limitation of the intervention evaluation is the planned experimental setting in which the study took place. Although using a sample drawn from Praeventis enabled us to test the effects of the intervention under more naturalistic conditions, these were not entirely natural. After all, in order to participate in the study, mothers had to provide informed consent. This means that they were aware of the purpose of the study (i.e., improving HPV vaccination acceptability). We believe a natural experiment could have added value since this enables the intervention to be evaluated beyond those that were amenable to the planned experiment (Craig et al., 2008). This could be realized by conducting another extensive effect evaluation when the intervention has successfully been implemented nationally.

The intervention evaluation also had important strengths. First, we incorporated both an effect and process evaluation. The latter is often overlooked, but provided us valuable insight about the implementation and working mechanisms of the intervention. Second, we included an objective measurement of HPV vaccination uptake (as derived from Praeventis). Third, we had a broad focus on outcomes (i.e., determinants of HPV vaccination acceptance, uptake, informed decision-making, and decisional conflict). And, finally, by including a naturalistic sample in the study (next to a controlled sample), we were able to demonstrate that the positive intervention effects were also found under naturalistic conditions. Therefore, these positive effects are likely to be translated into practice once the intervention has become part of the national implementation strategy.

\section{RECOMMENDATIONS FOR FUTURE PRACTICE}

The Web-based, tailored intervention that was described in this thesis has shown the potential to improve HPV vaccination acceptability and informed decision making, decrease decisional conflict, as well as motivate HPV vaccination uptake among mothers 
of invited girls. To our knowledge, this is the first online tailored intervention to have found such positive effects on HPV vaccination acceptance. In addition, the intervention was positively evaluated by the mothers. Taken together, we believe that the intervention we developed is suitable for broad nationwide dissemination and implementation. From 2019 on, the intervention will be incorporated into the HPV vaccination communication. We do reckon that attention should be paid to the dissemination of the intervention, especially among specific subgroups that were shown less likely to be reached (i.e., those with a low educational level, those born outside the Netherlands, and those who are less likely to vaccinate their daughter against HPV). A suggestion for doing this is by promoting the intervention, for instance on social media or on websites that have been shown to be frequently visited by these mothers. Next, we will discuss recommendations for future practices successively derived from our experience with the needs assessment, intervention development, and intervention evaluation.

\section{PART I: The Needs assessment}

Although we selected mothers as the target group for the intervention, this does not imply that interventions promoting HPV vaccination acceptance should only focus on the mothers. Girls are an important target group as well. After all, in the Netherlands, girls aged 12 are legally entitled to make their own decision about their HPV vaccination. And also, girls expressed their need for interactive and personal information about the HPV vaccination (Van Keulen et al., 2010). In addition, the opinion of the daughter and / or partner of the mother appeared to be an important constituent for the mothers' decision about the HPV vaccination of the daughter (Chapter 2). Besides, boys may also become a relevant target group in the near future. In other countries, such as Australia, boys are already included in the national immunization program. In the Netherlands, this is a current topic being discussed by the Dutch Health Council, since it may contribute to the achievement of herd immunity and to a reduction of the global burden of a variety of HPV-related cancers in women and men (Braakhuis, Leemans, \& Visser, 2014; Georgousakis et al., 2012; Wakeham \& Kavanagh, 2014).

We recommend future interventions promoting HPV vaccination acceptance to target attitude, beliefs, subjective norms, relative effectiveness, anticipated regret, risk perception, self-efficacy, and knowledge. Especially attitude, since HPV vaccination decision making is for the most attitude-driven (Chapter 2). We suggest future interventions to be tailored to the following behavioral beliefs: beliefs about (1) the role the government takes regarding HPV vaccination, (2) the daughters' sexual behavior and maturation relative to the need for the HPV vaccination, (3) the safety and effectiveness of the HPV vaccination, and (4) the impact of pharmaceutical industries on national immunization policies. Furthermore, 
we recommend future interventions to detect potential misperceptions regarding the HPV vaccination and, subsequently, correct them. This enables mothers to make an informed decision, based on correct information. For instance, we discovered misperceptions regarding the relative effectiveness of the HPV vaccination compared to other methods (Chapter 2) and regarding HPV-related perceived risks (Chapter 3).

Another recommendation concerns adding strategies that might decrease the intentionbehavior gap (57\% unexplained variance) (Chapter 2). An example of such a strategy is the recall and reminder system (e.g., phone calls, text messages by phone, (e-mailed letters, and/or outreach visits), which has been shown to be one of the most effective single-method strategies in improving uptake according to several reviews (Fu et al., 2014; Niccolai \& Hansen, 2015; Smulian, Mitchell, \& Stockley, 2016; Walling et al., 2016). Another way of decreasing the gap might be a change in the mode of delivering the HPV vaccination. Currently, in the Netherlands, the HPV vaccination is given at a set timepoint at predetermined public venues (e.g., sports centers). This restricts the opportunity for interaction between the parent/girl with the professional. A school-based approach provides more room for personal interaction. We believe that a combination of face-to-face and internet delivery increases commitment to the intervention, which is likely to stimulate girls and parents to actually get the HPV vaccination once they have decided to do so. Support for this notion comes from a study of Grandahl and colleagues (2016), who found that an intervention delivered individually, face-to-face by school nurses positively affected beliefs towards HPV prevention as well as vaccination uptake. Moreover, a school-based approach eliminates the potential barrier of girls and/or their parents being be unable to get the vaccination at the specific time and/or location (e.g., parents may have to work or the location is difficult to reach). After all, in general, girls attend school on a daily basis (Fererico et al., 2010).

\section{PART II: Intervention Development}

Future interventions promoting HPV vaccination acceptance should be developed systematically (e.g., by applying the IM protocol). This ensures a solid theoretical and empirical foundation for the intervention and maximizes the likelihood of the intervention being effective (cf. Bartholomew Eldredge et al., 2016). Also, this makes the process of intervention development explicit and transparent, thereby providing a road map of the decision-making process and its main outcomes. This, in turn, provides leads for the development of other interventions. For instance, similar interventions could be developed for the girls themselves, as they also expressed their need for interactive and personal information about the HPV vaccination (Van Keulen et al, 2010). This still needs to be investigated for the partners (see 'Recommendations for future research'). Also, if the 
government decides to include boys, interventions may need to be developed to enhance HPV vaccination acceptance among boys and to make sure that they (and their parents) are well informed. The detailed, comprehensive description of intervention development that we provided in Chapter 4, can serve as a roadmap for this.

Moreover, we recommend to (systematically) develop similar intervention-models for the other childhood vaccinations. Also, new vaccinations have become available for specific subgroups, not yet included in the NIP. The Dutch Health Council stated that potential health gains are being left untapped, because the uptake of these new vaccines remains to be insufficient (Gezondheidsraad, 2013). Therefore, the RIVM conducted research into the differential needs for information among the public and the professionals. An example of such a vaccine is maternal vaccination against pertussis, which is considered to be the most successful and effective intervention to prevent whooping cough among babies (Fernández-Cano et al., 2015; Forsyth et al., 2015). It was found by the RIVM that mothers considered it to be essential to make an informed decision before getting the vaccination (Lehmann, 2017). Factors that were shown to play a role in this decision were, for example, risk perception and effectiveness and safety of the vaccination. This is comparable to what we (and others) found in the field of HPV vaccination.

Furthermore, although computer-tailoring was already used throughout the intervention in several ways, we believe that room for improvement can be found at the level of tailoring. In our intervention, we solely used a personalized tailored approach. However, Hopfer (2012), demonstrated that among college-aged women, a culturally tailored intervention (i.e., a narrative video) doubled HPV vaccination uptake compared to controls. Cultural tailoring can be defined as the use of health messages '..which recognize and reinforce a group's cultural values, beliefs, and behaviors built upon those to provide context and meaning to the health message' (Resnicow et al., 2002). Future interventions promoting HPV vaccination acceptance could incorporate both personal and cultural tailoring, for instance by tailoring on cultural factors such as religious rules and values and responsibility (Hopfer, 2012). In addition, we believe the level of tailoring of the virtual assistants could be improved. Durantini and colleagues (2006) has shown that tailoring a virtual agent to demographic and behavioral similarities, facilitates behavior change.

\section{PART III: Intervention Evaluation}

We recommend future interventions to test both the intervention's efficacy and effectiveness since we consider both types to be useful. After all, the most effective intervention will not have public impact if the positive intervention effects found in an efficacy trial, disappear under natural conditions. This could be done, for instance, by conducting two trials (one testing efficacy and one testing effectiveness) or by conducting a single trial using two 
differential samples (one controlled sample for efficacy testing and one naturalistic sample for effectiveness testing, which we did). Another recommendation is to include a broad scope of outcomes, including actual HPV vaccination uptake. So far, few interventions promoting HPV vaccination acceptance have utilized behavioral outcomes (for a review, see Priest \& Knowlden, 2015). Moreover, we recommend to assess uptake objectively, since self-reports might be subject to bias.

We also recommend future interventions promoting HPV vaccination acceptance to incorporate a process evaluation next to the effect evaluation. After all, in our study, the process evaluation enabled us to detect the positive effect of exposure to the intervention on outcome measures, including actual HPV vaccination uptake (Hofstra, 2017). It also enabled us to draw conclusions about whether the intervention was appreciated by the target group. However, especially in the field of promoting HPV vaccination acceptance, there are few interventions that have incorporated a process evaluation so far (Priest \& Knowlden, 2015).

Although the process evaluation showed objective program use to be relatively high, we reckon that this could be further improved. Online intervention use can be divided into three phases: a first visit, staying, and revisiting (Crutzen et al., 2008; Van't Riet, Crutzen, \& De Vries, 2010). In the context of decision-making about the HPV vaccination, we consider revisiting to be optional, but not necessary since getting the HPV vaccination is a nonrepetitive behavior. Program use in terms of a both a first visit and staying thereafter could be improved by arousing interest. Support for this notion comes from a sequence of studies conducted by Crutzen, Ruiter, and De Vries (2014). They showed that arousing interest successfully increased the intention to visit a website about Hepatitis A, B, and C virus and the likelihood of clicking on the link to visit the website. Interest was aroused in the invitation by challenging the potential visitor regarding his knowledge about Hepatitis and it was emphasized that the website provided this information in a comprehensible manner. In addition, arousing interest on the website itself in a similar way (i.e., challenging questions above each paragraphs), increased the number of pages visited (Crutzen, Ruiter, \& De Vries, 2014). This is promising, since we found positive effects of the number of pages visited on several important outcomes (i.e., informed decision-making, HPV vaccination intention, and uptake) (Hofstra, 2017). Overall, we recommend future interventions promoting HPV vaccination acceptance to include strategies to arouse interest such as the one described above, both in the invitation and in the intervention itself. 


\section{RECOMMENDATIONS FOR FUTURE RESEARCH}

First, we recommend future research to conduct a needs assessment for relevant target groups other than the mothers. This has already been done for the girls by Van Keulen and colleagues (2013a), but this still needs to be conducted for the partners of the mothers. Besides, if the government decides to include vaccinating boys against HPV into the NIP, this is also recommended for both the boys and their parents. Results from such needs assessments, in turn, can be used for intervention development targeting these specific groups.

Second, in the analyses, the construct decisional conflict acted strangely. Specifically, we found that the positive univariate association with HPV vaccination intention turned negative in the multivariate model (Chapter 2). This has also been found by others and is likely to indicate a suppressor-effect (Van Keulen et al., 2013a). A suppressor is a variable that when added to the model, raises observed R-square mostly due to its correlation with other independent variables. However, this is not due to its own association with the dependent variable, which is comparatively weak (Maassen \& Bakker, 2001). This raised the question whether decisional conflict can be conceived as a predictor of HPV vaccination uptake (which we did in Chapter 2). In retrospect, we believe the construct better fits as an outcome of the decision-making process about the HPV vaccination, since decisional conflict may arise when feeling uninformed (O'Connor, Jacobsen, \& Stacey, 2002). Therefore, we changed our perspective of seeing decisional conflict as a determinant (Chapter 2) into seeing it as an outcome of the decision-making process regarding the HPV vaccination. This was the reason for adding the intervention objective of decreasing decisional conflict (next to improving HPV vaccination uptake and informed decision-making) (Chapters 4-5). Future studies aimed at predicting health behavior should be cautious when there is an indication for a suppressor-effect and take action if needed (e.g., by removing the suppressor from the model).

Third, we recommend future studies to include a continuous measure for informed decision-making. Informed decision-making is usually expressed dichotomously (e.g., see Van der Pal, Otten, \& Detmar, 2010). However, we agree with others that dichotomizing may lead to several problems (Altman \& Royston, 2006). For instance, information gets lost by dichotomizing, so, the power to detect an association between the variable and the outcome is reduced (Austin \& Brunner, 2004). After all, the selection of the cut-off points is somewhat arbitrary, yet critical for the outcome (Altman \& Royston, 2006). 
In retrospect, we question whether "habit" is a suitable construct when trying to explain HPV vaccination uptake. Habits can be defined as '... learned sequences of acts that he become automatic responses to specific cues, and are functional in obtaining certain goals or end states' (Verplanken \& Aarts, 1999, p.104). Habits are formed through repetition of behaviour in a specific context (Lally et al., 2010). Thus, the more frequently we perform a behavior, the more likely it is to become habitual. For instance, habit of past influenza vaccination behavior has been shown to be a strong predictor of the intention to receive influenza vaccine in a subsequent year (Lin et al., 2010). In our study, we measured habit specifically as the habit to get the HPV vaccination. This behavior, however, is not considered to be a repetitive behavior, since girls only have to complete one series (i.e., two injections) once. But, it could be that the habit of vaccinating in general plays a role in the decision about the HPV vaccination. Mothers could have received the invitation to the HPV vaccination and automatically decide to have their daughter against HPV, since they have done this as well for the other vaccinations offered by the NIP (i.e., childhood vaccinations). Although this might be the case for some, it appears to be unlikely for others, as can be seen in the discrepancy in the uptake between the childhood vaccinations (around 95\%) and the HPV vaccination at the introduction of the HPV program (52\%). This could be explained by the fact that the HPV vaccination differs from the childhood vaccinations. For instance, the HPV vaccination offers protection against a sexually transmitted disease, which might be a sensitive topic. Future studies are needed to investigate if and how the construct habit fits within context of HPV vaccination.

Furthermore, we recommended to change the mode of delivery into a school-based approach to increase the intention-behavior gap (see 'Recommendations for future interventions'). Future studies are needed whether this approach has added value over the current mode of delivery (i.e., mass vaccination).

\section{GENERAL CONCLUSION}

This $\mathrm{PhD}$ thesis was about the systematic development and evaluation of an interactive, Web-based, tailored intervention with virtual assistants promoting HPV vaccination acceptability among mothers of invited girls. We have demonstrated that the intervention has potential to improve both HPV vaccination acceptability and informed decision making, and to decrease decisional conflict. Also, it may increase actual uptake when the mothers' exposure to the intervention is higher. We therefore conclude that this thesis has contributed to the improvement of the communication about the HPV vaccination. From 2019 on, the Web-based tailored intervention will be incorporated into the national HPV vaccination communication. 



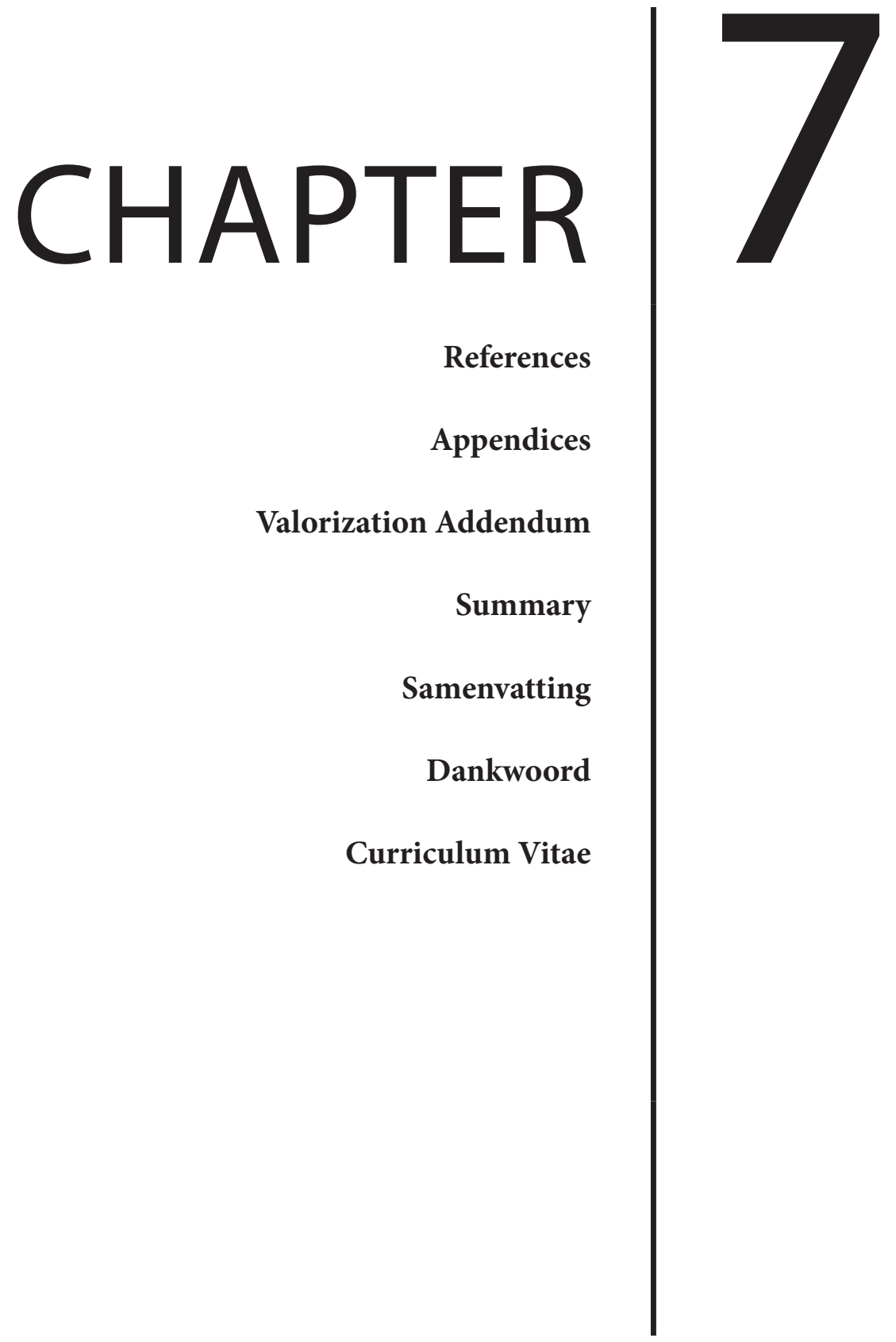





\section{REFERENCES}

Ajzen, I. (1985). From intentions to actions: A theory of planned behavior. In Kuhl, J., \& Beckmann, J. (Eds.). Action-control: From cognition to behavior (pp. 1 1-39). Heidelberg, Germany: Springer. Ajzen, I. (1991). The theory of planned behavior. Organizational Behavior and Human Decision Processes, 50, 179-211.

Alberts, C., Schim van der Loeff, M.F., Hazeveld, Y., de Melker, H.E., van der Wal, M.F., Nielen, ... Paulussen, T.G.W.M. (2017). A longitudinal study on determinants of HPV vaccination uptake in parents/guardians from different ethnic backgrounds in Amsterdam, the Netherlands. BMC Public Health, 17, 220. doi: 10.1186/s12889-017-4091-4

Allen, M. (1991). Meta-analysis comparing the persuasiveness of one-sided and two-sided messages. Western Journal of Communication, 55(4), 390-404. doi: 10.1080/10570319109374395

Altman, D.G., \& Royston P. (2006). The cost of dichotomising continuous variables. British Medical Journal, 332, 1080. doi: 10.1136/bmj.332.7549.1080

Arrossi, S., Temin, S., Garland, S.M., O’Neal Eckert, L., Bhatla, N., \& Castellsaguét, X. (2017). Primary prevention of cervical cancer: American Society of Clinical Oncology resource-stratified guideline. Journal of Global Oncology, 3, 611-634. doi: 10.1200/JGO.2016.008151

Atkinson, R.K. (2002). Optimizing learning from examples using animated pedagogical agents. Journal of Educational Psychology, 94, 416-427. doi: 10.1037/0022-0663.94.2.416

Austin, P.C., \& Brunner, L.J. (2004). Inflation of the type I error rate when a continuous confounding variable is categorized in logistic regression analyses. Statistics in Medicine, 23(7), 1159-1178.

Bandura, A. (1986). Social foundations of thought and action: A social cognitive theory. New York: Prentice Hall.

Bartholomew Eldridge, L.K., Markham, C.M., Ruiter, R.A.C., Fernàndez, M.E., Kok, G., \& Parcel, G.S. (2016). Planning health promotion programs: An Intervention Mapping approach (4th ed.). Hoboken, NJ: Wiley. ISBN: 978-1119035497

Bartholomew, L.K., \& Mullen, P.D. (2011). Five roles for using theory and evidence in the design and testing of behavior change interventions. Journal of Public Health Dentistry, 71, S20-S33.

Baylor, A.L. (2009). Promoting motivation with virtual agents and avatars: Role of visual presence and appearance. Philosophical Transactions of the Royal Society of London, 364, 3559-3565. doi: 10.1098/rstb.2009.0148

Baylor, A.L. (2011). The design of motivational agents and avatars. Educational Technology Research and Development, 59, 291-300. doi: 10.1007/s11423-011-9196-3

Baylor, A.L., \& Kim, Y. (2004). Pedagogical agent design: the impact of agent realism, gender, ethnicity, and instructional role. In: LNCS, intelligent tutoring systems (vol. 3320, pp. 592-603). Berlin/ Heidelberg, Germany: Springer.

Becker, M.H. (1974). The health belief model and personal health behaviour. Health Education Monographs, 30, 324-508.

Bekkers, R.L., Massuger, L.F., Bulten, J., \& Melchers, W.J. (2004). Epidemiological and clinical aspects of human papillomavirus detection in the prevention of cervical cancer. Reviews in Medical Virology, 14(2), 95-105. doi: 10.1002/rmv.416 
Bennett, A.T., Patel, D.A., Carlos, R.C., Zochowski, M.K., Pennewell, S.M., Chi, A.M., \& Dalton, V.K. (2015). Human papillomavirus vaccine uptake after a tailored, online educational intervention for female university students: a randomized controlled trial. Journal of Women's Health, 24(11), 950-957. doi:10.1089/jwh.2015.5251

Betsch, C., Renkewitz, F., Betsch, T., \& Ulshöfer, C. (2010). The influence of vaccine-critical websites on perceiving vaccination risks. Journal of Health Psychology, 15(3), 446-455. doi: $10.1177 / 1359105309353647$

Beun, R.J., de Vos, E., \& Witteman, C. (2003). Embodied conversational agents: Effects on memory performance and anthropomorphisation. Proceedings of the International Conference on Intelligent Virtual Agents (pp. 315-319). Berlin/Heidelberg, Germany: Springer.

Bickmore, T.W., \& Picard, R.W. (2005). Establishing and maintaining long-term human-computer relationships. ACM Transactions on Computer-Human Interaction, 12(2), 293-327. doi: $10.1145 / 1067860.1067867$

Bickmore, T., Puskar, K., Schlenk, E., Pfeifer, L., \& Sereika, S. (2010). Maintaining Reality: Relational Agents for Antipsychotic Medication Adherence. Interacting with computers, 22(4), 276. doi: 10.1016/j.intcom.2010.02.001

Biesecker, B.B., Schwartz, M.D., \& Marteau, T.M. (2013). Enhancing informed choice to undergo health screening: A Systematic Review. American Journal of Health Behavior, 37, 351-359. doi: 10.5993/AJHB.37.3.8

Blanson Henkemans, O.A., Rogers, W.A., Fisk, A.D., Neerincx, M.A., Lindenberg, J., \& van der Mast, C.A.P.G. (2008). Usability of an Adaptive Computer Assistant that Improves Self-Care and Health Literacy of Older Adults. Special Issue Methods of Information in Medicine Smart Homes and Ambient Assisted Living in an Aging Society, 47, 82-88.

Blanson Henkemans, O.A., van der Boog, P.J., Lindenberg, J., van der Mast, C.A., Neerincx, M.A., \& Zwetsloot-Schonk, B.J. (2009). An online lifestyle diary with a persuasive computer assistant providing feedback on self-management. Technology and Health Care, 17, 253-267. doi: 10.3233/ THC-2009-0545

Block, L.G., \& Williams, P. (2002). Undoing the effects of seizing and freezing: decreasing defensive processing of personally relevant messages. Journal of Applied Social Psychology, 32(4), 803-833. doi: 10.1111/j.1559-1816.2002.tb00243.x

Bosch, F.X., Lorincz, A., Munoz, N., Meijer, C.J. \& Shah, K.V. (2002). The causal relation between human papillomavirus and cervical cancer. Journal of Clinical Pathology, 55, 244-265.

Bowling A. (2005). Just one question: if one question works, why ask several? Journal of Epidemiology and Community Health, 59(5), 342-345. doi: 10.1136/jech.2004.021204

Braakhuis, B.J., Leemans, C.R., \& Visser, O. (2014). Incidence and survival trends of head and neck squamous cell carcinoma in the Netherlands between 1989 and 2011. Oral oncology, 50, 670675. doi: 10.1016/j.oraloncology.2014.03.008

Braspenning, J., Tacken,M., Penders, A., Van den Hoogen, H., \& De Bakker, D. (2001). Opkomst bevolkingsonderzoek baarmoederhalskanker, 1997-1999. TSG, 79, 341-345.

Breakwell, G.M. (2000). Risk communication: Factors affecting impact. British Medical Bulletin, 56(1), 110-120. doi: 10.1258/0007142001902824 
Brendryen, H., Johansen, A., Nesvag, S., Kok, G., \& Duckert, F. (2013). Constructing a theory- and evidence-based treatment rationale for complex eHealth interventions: development of an online alcohol intervention using an intervention mapping approach. JMIR Research Protocols, 2(1), e6. doi: 10.2196/resprot.2371

Brewer, N.T., Chapman, G.B., Gibbons, F.X., Gerrard, M., \& McCaul, K.D. (2007). Meta-analysis of the relationship between risk perception and health behavior: The example of vaccination. Health Psychology, 26, 136-145. doi: 10.1037/0278-6133.26.2.136

Brewer, N.T., \& Fazekas, K.I. (2007). Predictors of HPV vaccine acceptability: a theory-informed, systematic review. Preventive Medicine, 45(2-3), 107-114. doi: 10.1016/j.ypmed.2007.05.013

Brouwer, W., Kroeze, W., Crutzen, R., de Nooijer, J., de Vries, N.K., Brug, J., \& Oenema, A. (2011). Which intervention characteristics are related to more exposure to internet-delivered healthy lifestyle promotion interventions? A systematic review. Journal of Medical Internet Research, 13(1), e2. doi: 10.2196/jmir.1639

Brug, J., Oenema, A., \& Campbell, M. (2003). Past, present, and future of computer-tailored nutrition education. American Journal of Clinical Nutrition, 77, 1028-1034S. doi: 10.1093/ajcn/77.4.1028S

Bruni, L., Barrionuevo-Rosas, L., Albero, G., Serrano, B., Mena, M., Gómez, D., ... de Sanjosé, S. (2016). ICO Information Centre on HPV and Cancer (HPV Information Centre). Human Papillomavirus and Related Diseases in Netherlands. Summary Report 23 December 2016. Retrieved from: http://www.hpvcentre.net/statistics/reports/XWX.pdf

Carroll, C., Patterson, M., Wood, S., Booth, A., Rick, J., \& Balain, S. (2007). A conceptual framework for implementation fidelity. Implementation Science, 2, 40. doi: 10.1186/1748-5908-2-40.

Cafazzo, J.A., Leonard, K., Easty, A.C., Rossos, P.G., \& Chan, C.T. (2009). The User-Centered Approach in the Development of a Complex Hospital-at Home Intervention. Studies in Health Technology and Informatics, 143, 328-333.

Castellsagué, X., Muñoz, N., Pitisuttithum, P., Tresukosol, D., Monsonego, J., Ault, K., ... Saah, A. (2011). End-of-study safety, immunogenicity, and efficacy of quadrivalent HPV (types 6, 11, 16,18 ) recombinant vaccine in adult women $24-45$ years of age. British Journal of Cancer, 105, 28-37. doi: 10.1038/bjc.2011.185

Centraal Bureau voor de Statistiek. (2016a). Bevolking per maand; 20 tot 65 jarigen; vrouwen; herkomst; alle generaties. Retrieved from: http://statline.cbs.nl/Statweb/publication/?DM=SL $\mathrm{NL} \& \mathrm{PA}=71090 \mathrm{ned} \& \mathrm{D} 1=0 \& \mathrm{D} 2=1 \& \mathrm{D} 3=0,121 \& \mathrm{D} 4=0-1 \& \mathrm{D} 5=0 \& \mathrm{D} 6=4-6,52-54,112-114,124$ $126 \& \mathrm{HDR}=\mathrm{T}, \mathrm{G} 3 \& \mathrm{STB}=\mathrm{G} 2, \mathrm{G} 4, \mathrm{G} 5, \mathrm{G} 1 \& \mathrm{VW}=\mathrm{T}$

Centraal Bureau voor de Statistiek. (2016b). Bevolking; hoogst behaald onderwijsniveau; vrouwen; 25 tot 65 jaar. Retrieved from: http://statline.cbs.nl/Statweb/publication/?DM=SLNL\&PA=822 75 ned $\& D 1=0 \& D 2=1 \& D 3=3-6 \& D 4=0-1,4-5 \& D 5=0,4-5,8,12-13 \& D 6=60-66 \& H D R=T, G 1, G 3, G$ $5 \& \mathrm{STB}=\mathrm{G} 2, \mathrm{G} 4 \& \mathrm{VW}=\mathrm{T}$

Centraal Bureau voor de Statistiek. (2015). Religieuze betrokkenheid van bevolkingsgroepen, 20102014. Retrieved from: https://www.cbs.nl/-/media/imported/documents/2015/20/2015bt11religieuze-betrokkenheid-van-bevolkingsgroepen-20102014.pdf

Centers for Disease Control and Prevention. (2012). Human papillomavirus-associated cancersUnited States, 2004-2008. Morbidity and Mortality Weekly Report, 61(15), 258-261. 
Chaiken, S. (1980). Heuristic versus systematic information processing and the use of source versus message cues in persuasion. Journal of Personality and Social Psychology, 39, 752-766. doi: 10.1037/0022-3514.39.5.752

Chaturvedi, A.K., Engels, E.A., Pfeiffer, R.M., Hernandez, B.Y., Xiao, W., Kim, E., ... Gillison, M.L. (2011). Human papillomavirus and rising oropharyngeal cancer incidence in the United States. Journal of Clinical Oncology, 29(32), 4294-4301. doi: 10.1200/JCO.2011.36.4596

Christensen, H., Griffiths, K.M., \& Korten, A. (2002). Web-based cognitive behavior therapy: analysis of site usage and changes in depression and anxiety scores. Journal of Medical Internet Research, 4(1), e3. doi: 10.2196/jmir.4.1.e3

Cialdini, R.B., Demaine, L.J., Sagarin, B.J., Barrett, D.W., Rhoads, K., \& Winter, P.L. (2006). Managing social norms for persuasive impact. Social Influence, 1, 3-15. doi: 10.1080/15534510500181459

Cohen, J. (1988). Statistical power analysis for the behavioral sciences (2nd ed.). Hillsdale, NJ: Lawrence Earlbaum Associates.

Cohen, J. (2001). Defining identification: A theoretical look at the identification of audiences with media characters. Mass Communication and Society, 4, 245-264. doi: 10.1207/S15327825MCS0403_01

Côté, J.C., Godin, G., Garcia, P.L., Gagnon, M., \& Rouleau, G. (2008). Program development for enhancing adherence to antiretroviral therapy among persons living with HIV. Aids Patient Care STDS, 22, 965-975. doi: 10.1089/apc.2008.0124

Cox, D.S., Cox, A.D., Sturm, L., \& Zimet, G. (2010). Behavioral interventions to increase HPV vaccination acceptability among mothers of young girls. Health Psychology, 29(1), 29-39. doi: 10.1037/a0016942

Craig, P., Dieppe, P., Macintyre, S., Michie, S., Nazareth, I., \& Pettigrew, M. (2008).

Developing and evaluating complex interventions: the new Medical Research Council

guidance. British Medical Journal, 337, a1655. doi: 10.1136/bmj.a1655

Crutzen, R., de Nooijer, J., Brouwer, W., Oenema, A., Brug, J., \& De Vries, N.K. (2008). Internetdelivered interventions aimed at adolescents: a Delphi study on dissemination and exposure. Health Education Research, 23(3), 427-439. doi: 10.1093/her/cym094

Crutzen, R., Cyr, D., \& De Vries, N.K. (2012). The role of user control in adherence to and knowledge gained from a website: randomized comparison between a tunneled version and a freedom-ofchoice version. Journal of Medical Internet Research, 14, e45. doi: 10.2196/jmir.1922

Crutzen, R., Kohl, L., \& De Vries, N.K. (2013). Kennissynsthese online preventie.

Dahlstrom, L.A., Tran, T.N., Lundholm, C., Young, C., Sundstrom, K., \& Sparten, P. (2010). Attitudes to HPV vaccination among parents of children aged 12-15 years - A population-based survey in Sweden. International Journal of Cancer, 126(2), 500-507. doi: 10.1002/ijc.24712

Danaher, B.G., McKay, H.G., \& Seeley, J.R. (2005). The information architecture of behavior change websites. Journal of Medical Internet Research, 7, e12. doi: 10.2196/jmir.7.2.e12

Das, J.K., Salam, R.A., Arshad, A., Lassi, Z.S., \& Bhutta, Z.A. (2016). Systematic review and metaanalysis of interventions to improve access and coverage of adolescent immunizations. Journal of Adolescent Health, 59(4), S40-S48. doi: 10.1016/j.jadohealth.2016.07.005 
David, M.P., Van Herck, K., Hardt, K., Tibaldi, F., Dubin, G., Descamps, D., \& Van Damme, P. (2009). Long-term persistence of anti-HPV-16 and -18 antibodies induced by vaccination with the AS04-adjuvanted cervical cancer vaccine: Modeling of sustained antibody responses. Gynecologic Oncology, 115, S1-S6.

De Graaf, A., Hoeken, H., Sanders, J., \& Beentjes, J.W.J. (2012). Identification as a mechanism of narrative persuasion. Communication Research, 39, 802-823. doi: 10.1177/0093650211408594

De Graaf, H., Van der Borne, M., Nikkelen, S., Twisk, D., \& Meijer, S. (2017). Seks onder je 25e. Seksuele gezondheid van jongeren in Nederland anno 2017 / Sex under the age of 25. A population study of Dutch adolescent's sexual health. Delft: Eburon.

De Wit, J.B.F., Das, E., \& Vet, R. (2008). What works best: Objective statistics or a personal testimonial? An assessment of the persuasive effects of different types of message evidence on risk perception. Health Psychology, 27, 110-115. doi:10.1037/0278-6133.27.1.110

Dombrovski, S.U., Sniehotta, F.F., Avenell, A., \& Coyne, J.C. (2007). Towards a cumulative science of behaviour change: do current conduct and reporting of behavioural interventions fall short of best practice? Psychology and Health, 22(8), 869-874. doi: 10.1080/08870440701520973

Doorbar, J., Quint, W., Banks, L., Bravo, I.G., Stoler, M., Broker, T.R., \& Stanley, M.A. (2012). The biology and life-cycle of human papillomaviruses. Vaccine, 30(5), F55-F70. doi: 10.1016/j. vaccine.2012.06.083

Drozd, F., Haga, S.M., Brendryen, H., \& Slinning, K. (2015). An Internet-based intervention (Mamma $\mathrm{Mia}$ ) for postpartum depression: mapping the development from theory to practice. JMIR Research Protocols, 4(4), e120. doi: 10.2196/resprot.4858

Durantini, M.R., Albarracin, D., Mitchell, A.L., Earl, A.N., \& Gillette, J.C. (2006). Conceptualizing the influence of social agents of behavior change: A meta-analysis of the effectiveness of HIVprevention interventionists for different groups. Psychological Bulletin, 132(2), 212-248. doi: 10.1037/0033-2909.132.2.212

Eagly, A.H., \& Chaiken, S. (1993). The psychology of attitudes. Fort Worth, Texas: Harcourt Brace Jovanovich.

Einstein, M.H., Schiller, J.T., Viscidi, R.P., Strickler, H.D., Coursaget, P., Tan, T., ... Jenkins D. (2009). Clinican's guide to human papillomavirus immunology: knows and unknowns. Lancet Infectious Diseases, 9, 347-356. doi: 10.1016/S1473-3099(09)70108-2.

Ernst, E., \& Pittler, M.H. (2006). Efficacy or effectiveness? Journal of General Internal Medicine, 260, 488-490. doi: 10.1111/j.1365-2796.2006.01707.x

European Medicines Agency. (2014). EPAR summary for the public. Secondary EPAR summary for the public 2014. Retrieved from:

http://www.ema.europa.eu/docs/en_GB/document_library/EPAR_-_Summary_for_the_public/ human/000703/WC500021146.pdf

Eurostat. (2017). Internet access and use statistics - households and individuals. Retrieved from: http:// ec.europa.eu/eurostat/statistics-explained/index.php/Internet_access_and_use_statistics_-_ households_and_individuals

Eysenbach, G. (2005). The law of attrition. Journal of Medical Internet Research, 7(1), e11. doi: 10.2196/ jmir.7.1.e11 
Fayers, P.M., \& King, M.T. (2009). How to guarantee finding a statistically significant difference: The use and abuse of subgroup analyses. Quality of Life Research, 18, 527-530. doi: 10.1007/s11136009-9473-3

Fererico, S.G., Abrams, L., Everhart, R.M., Melinkovich, P., \& Hambridge, S.J. (2010). Addressing adolescent immunization disparities: a retrospective analysis of school-based health center immunization delivery. American Journal of Public Health, 100(9), 1630-1634. doi: 10.2105/ AJPH.2009.176628

Ferlay, J., Shin, H.R., Bray, F., Forman, D., Mathers, C., \& Parkin, D.M. (2010). Estimates of worldwide burden of cancer in 2008. International Journal of Cancer, 127(12), 2893-2917. doi: 10.1002/ ijc. 25516

Fernández-Cano, M.I., Armadans, Gil, L., \& Campins Marti, M. (2015). Vaccine, 33(19), 2213-2220. doi: 10.1016/j.vaccine.2015.03.045

Fishbein, M., \& Ajzen, I. (1975). Beliefs, Attitude, Intention and Behaviour: An Introduction to Theory and Research. Reading, MA: Addison-Wesley.

Fishbein M., \& Ajzen, I. (2010). Predicting and changing behavior: The Reasoned Action Approach. New York: Taylor \& Francis.

Fischhoff, B., Bostrom. A., \& Quadrell, M. J. (1993). Risk perception and communication. Annual Review of Public Health, 4, 183-203. doi: 10.1146/annurev.pu.14.050193.001151

Forsyth, K., Plotkin, S., Tan, T., \& Wirsing von König, C.H. (2015). Strategies to decrease pertussis transmission to infants. Pediatrics, 135(6), e1475-82. doi: 10.1542/peds.2014-3925

Franco, E.L., Duarte-Franco, E., \& Ferenczy, A. (2001). Cervical cancer: epidemiology, prevention and the role of human papillomavirus infection. Canadian Medical Association Journal, 164, 1017-1025.

Fu, L.Y., Bonhomme, L.A., Cooper, S.C., Joseph, J.G., \& Zimet, G.D. (2014). Educational interventions to increase HPV vaccination acceptance: a systematic review. Vaccine, 32, 1901-1920. doi: 10.1016/j.vaccine.2014.01.091

Gardner, B., Whittington, C., McAteer, J., Eccles, M.P., \& Michie, S. (2010). Using theory to synthesise evidence from behaviour change interventions: the example of audit and feedback. Social Science \& Medicine, 70(10), 1618-1625. doi: 10.1016/j.socscimed.2010.01.039

Genefaite, G., Smit, M., Nijman, H.W., Tami, A., Drijfhout, I.H., Pascal, A., ... Hak, E. (2002). Comparatively low attendance during Human Pappilomavirus catch-up vaccination among teenage girls in the Netherlands: Insights from a behavioral survey among parents. BMC Public Health, 12, 498. doi: 10.1186/1471-2458-12-498

Georgousakis, M., Jayasinghe, S., Brotherton, J., Gilroy, N., Chiu, C., \& Macartney, K. (2012). Population-wide vaccination against human papillomavirus in adolescent boys: Australia as a case study. Lancet Infectious Diseases, 12, 627-634. doi: 10.1016/S1473-3099(12)70031-2

Gerend, M.A., Shepherd, M.A., \& Lustria, M.L. (2013). Increasing human papillomavirus vaccine acceptability by tailoring messages to young adult women's perceived barriers. Sexual Transmitted Diseases, 40(5), 401-405. doi: 10.1097/OLQ.0b013e318283c8a8 
Gerend, M.A., \& Shephard, J.E. (2012). Predicting human papillomavirus vaccine uptake in young adult women: Comparing the health belief model and theory of planned behavior. Annals of Behavioral Medicine, 44, 171-180. doi: 10.1007/s12160-012-9366-5

Gerend, M.A., Weibley, E.B.S., \& Bland, H.M.D. (2009). Parental response to Human Papillomavirus Vaccine availability: Uptake and intentions. Journal of Adolescent Health, 45(5), 528-531. doi: 10.1016/j.jadohealth.2009.02.006

Gezondheidsraad (2008). Vaccinatie tegen baarmoederhalskanker. Den Haag: Gezondheidsraad, 154. Gezondheidsraad (2013). Het individuele, collectieve en publieke belang van vaccinatie. Den Haag, 2013/21. ISBN 978-90-5549-973-1

Gilbert, D.T. (1991). How mental systems believe. American Psychology,46, 107-119. doi: 10.1037/0003066X.46.2.107

Gillison, M.L., Chaturvedi, A.K., \& Lowy, D.R. (2008). HPV prophylactic vaccines and the potential prevention of noncervical cancers in both men and women. Cancer, 113(10), 3036-3046. doi: $10.1002 / \mathrm{cncr} .23764$

Giuliano, A.R., Lee, J.H., Fulp, W., Villa, L.L., Lazcano, E., Papenfuss, M.R., ... Smith, D. (2011). Incidence and clearance of genital human pappilomavirus infection in men (HIM): a cohort study. Lancet, 377, 932-940 .doi: 10.1016/S0140-6736(10)62342-2

Giuliano, A.R., Nyitray, A.G., Kreimer, A.R., Pierce Campbell, C.M., Goodman, M.T., Staci, L., .... Franceschi, S. (2015). EUROGIN 2014 roadmap: Differences in human papillomavirus infection natural history, transmission and hu- 25 Introduction 1 man papillomavirus-related cancer incidence by gender and anatomic site of infection. International Journal of Cancer, 136, 2752-2760.

GLOBOCAN. (2012). Cervical Cancer Estimated Incidence, Mortality and Prevalence Worldwide in 2012. Retrieved from: http://globocan.iarc.fr/old/FactSheets/cancers/cervix-new.asp

Green, M.C., \& Brock, T.C. (2000). The role of transportation in the persuasiveness of public narratives. Journal of Personality and Social Psychology, 79, 701-721. doi: 10.1037/0022-3514.79.5.701.

Grandahl, M., Rosenblad, A., Stenhammar, C., Tydén, T., Westerling, R., Larsson, M., ... Nevéus, T. (2016). Schoolbased intervention for the prevention of HPV among adolescents: a randomised controlled study. BMJ Open, 6(1), e009875. doi: 10.1136/bmjopen-2015-009875

Gregory, W.L., Cialdini, R.B., \& Carpenter, K.M. (1982). Self-relevant scenarios as mediators of likelihood estimates and compliance: Does imagining make it so? Journal of Personality and Social Psychology, 43, 89-99.

Griffiths, F., Lindenmeyer, A., Powell, J., Lowe, P., \& Thorogood, M. (2006). Why are health care interventions delivered over the internet? A systematic review of the published literature. Journal of Medical Internet Research, 8(2), e10. doi: 10.2196/jmir.8.2.e10

Grimshaw, J. M., Presseau, J., Tetroe, J., Eccles, M.P., Francis, J.J., Godin, G., ... Zwarenstein, M. (2007). Looking inside the black box: A theory-based process evaluation alongside a randomised controlled trial of printed educational materials to improve referral and prescribing practices in primary care in Ontario, Canada. Implementation Science, 2(1), 38. doi: 10.1186/1748-5908-9-86

Hastie, T., Tibshirani, R., \& Friedman, J. (2001). The elements of statistical learning: Data mining, Inference, and Prediction. New York: Springer. 
Hayes, A. (2013). Introduction to mediation, moderation, and conditional process analysis: A regressionbased approach. New York: The Guilford Press.

Heinen, M.M., Bartholomew, L.K., Wensing, M., van de Kerkhof, P., \& van Achterberg, T. (2006). Supporting adherence and healthy lifestyles in leg ulcer patients: systematic development of the lively legs program for dermatology outpatient clinics. Patient Education and Counseling, 61, 279-291. doi: 10.1016/j.pec.2005.04.007

Hintze, J. (2004). NCSS and PASS. Number cruncher statistical systems. Kaysville: Utah. Available at www.ncss.com.

Hinyard, L., \& Kreuter, M.W. (2007). Using narrative communication as a tool for health behavior change: A conceptual, theoretical, and empirical overview. Health Education and Behavior, 34, 777-792. doi: 10.1177/1090198106291963

Hofman, R., Van Empelen, P., Richardus, J.H., de Kok, I.M.C.M., de Koning, H.J., van Ballegooijen, M., \& Korfage, I.J. (2014). Predictors of HPV vaccination uptake: a longitudinal study among parents. Health Education Research, 29(1): 83-96. doi: 10.1093/her/cyt092

Hopfer, S. (2012). Effects of a narrative HPV vaccination intervention aimed at reaching college women: A randomized controlled trial. Prevention Science, 13, 173-182. doi: 10.1007/s11121011-0254-1

Hofstra, M.C. (2017). Evaluating program adherence of online tailored education about the HPV vaccination. Master's thesis. Leiden: Leiden University.

IBM Corp. Released 2013. IBM SPSS Statistics for Windows, Version 22.0. Armonk, NY: IBM Corp. International agency for research on cancer (2005). Cervix Cancer screening. Lyon: IARC Press.

Integraal Kankercentrum Nederland (2013). Cijfers over kanker. Retrieved from: http://www. cijfersoverkanker.nl/selecties/Dataset_1/img5a37faea7b70b

Integraal Kankercentrum Nederland. (2016.) Cijfers over kanker. Retrieved from: http://www. cijfersoverkanker.nl/selecties/Dataset_1/img5a37faea7b70b

Janssen, E., van Osch, L., de Vries, H., \& Lechner, L. (2013). The influence of narrative risk communication on feelings of cancer risk. British Journal of Health Psychology, 18, 407-419. doi: 10.1111/j.2044-8287.2012.02098.x

Jerant, A., Kravitz, R.L., Rooney, M., Amerson, S., Kreuter, M., \& Franks, P.(2007). Effects of a tailored interactive multimedia computer program on determinants of colorectal cancer screening: a randomized controlled pilot study in physician offices. Patient Education and Counseling, 66, 67-74. doi : 10.1016/j.pec.2006.10.009

Jibaja-Weiss, M.L., Volk, R.J., Granch, T.S., Nefe, N.E., Spann, S.J., Aoki, N., ... Beck, J.R. (2006). Entertainment education for informed breast cancer treatment decisions in low-literate women: Development and initial evaluation of a patient decision aid. Journal of Cancer Educaction, 21, 133-139. doi : 10.1207/s15430154jce2103_8

Jin, S.A. (2010). The effects of incorporating a virtual agent in a computer-aided test designed for stress management education: the mediating role of enjoyment. Computers in Human Behavior, 26, 443-451. doi: 10.1016/j.chb.2009.12.003 
Juraskova, I., O'Brien, M., Mullan, B., Bari, R., Laidsaar-Powell, R., \& McCaffery, K. (2012). HPV vaccination and the effect of information framing on intentions and behaviour: an application of the theory of planned behaviour and moral norm. International Journal of Behavioral Medicine, 19(4), 518-25. doi: 10.1007/s12529-011-9182-5

Kahnemann, D., \& Tversky, A. (1982). The simulation heuristic. In D. Kahnemann, P. Slovic, \& A. Tversky (Eds.), Judgment under uncertainty: Heuristics and biases (pp. 201-208). Cambridge, MA: Cambridge University Press.

Kata, A. (2012). Anti-vaccine activists, Web 2.0, and the postmodern paradigm-An overview of tactics and tropes used online by the anti-vaccination movement. Vaccine, 30(25), 3778-3789. doi: 10.1016/j.vaccine.2011.11.112

Kessels, L.T.E., Harris, P.R., Ruiter, R.A.C., \& Klein, W.M.P. (2016). Attentional effects of selfaffirmation in response to graphic antismoking images. Health Psychology, 35(8), 891-897. doi: 10.1037/hea0000366

Kjaer, S.K., Chackerian, B., van den Brule, A.J., Svare, E.I., Paull, G., Walbomers, J.M., ... Meijer, C.L. (2001). High-risk Human Pappilomavirus is Sexually Transmitted: Evidence from a Follow-up study of Virgins Starting Sexual Activity (Intercourse). Cancer Epidemiology, Biomarkers, and Prevention, 10, 101-106.

Kjaer, S.K., Munk, C., Winter, J.F., Jørgensen, H.O., Meijer, C.J.L.M., \& van den Brule, A.J.C. (2005). Acquisition and Persistence of Human Pappilomavirus Infection in Younger Men: A Prospective follow-up Study among Danish Soldiers. Cancer Epidemiology, Biomarkers, and Prevention, 14, 1528-1533.

Kohl, L.F.M., Crutzen, R., \& de Vries, N.K. (2013). Online Prevention Aimed at Lifestyle Behaviors: A Systematic Review of Reviews. Journal of Medical Internet Research, 15(7):e146. doi: 10.2196/ jmir.2665

Kok, G., \& Mesters, I. (2011). Getting inside the black box of health promotion programmes using intervention Mapping. Chronic Illness, 7(3), 176-180. doi: 10.1177/1742395311403013

Koutsky, L.A., Holmes, K.K., Critchlow, C.W., Stevens, C.E., Paavonen, J., Beckmann, A.M., ... Kiviat, N.B. (1992). A cohort study of the risk of cervical intraepithelial neoplasia grade 2 or 3 in relation to papillomavirus infection. New England Journal of Medicine, 327, 1272-1278. doi: 10.1056/NEJM199210293271804

Krebs, P., Prochaska, J.O., \& Rossi, J.S. (2010). A meta-analysis of computer-tailored interventions for health behavior change. Preventive Medicine 51(3-4), 214-421. doi: 10.1016/j.ypmed.2010.06.004

Kreuter, M.W., Green, M.C., Cappella, J.N., Slater, M.D., Wise, M.E., Storey, D., ... Woolley, S. (2007). Narrative communication in cancer prevention and control: a framework to guide research and application. Annals of Behavioral Medicine, 33(3), 221-235. doi: 10.1080/08836610701357922

Kreuter, M.W., \& Wray, R.J. (2003). Tailored and targeted health communication: Strategies for enhancing information relevance. American Journal of Health Behavior, 27, S227-S232. doi: 10.5993/AJHB.27.1.s3.6

Labrie, J.W., Pederson, E.R., Earleywine, M., \& Olsen, H. (2006). Reducing heavy drinking in college males with the decisional balance: analyzing an element of motivational interviewing. Addictive Behaviors, 31, 254-263. doi: 10.1016/j.addbeh.2005.05.001 
Lally, P., Van Jaarsveld, C.H.M., Potts, H., \& Wardle, J. (2010). How are habits formed: Modeling habit formation in the real world. European Journal of Social Psychology, 40, 998-1009. doi:10.1002/ ejsp. 674

Lee, B.T., Chen, C., Yueh, J.H., Nguyen, M.D., Lin, S.J., \& Tobias, A.M. (2010). Computer-based learning module increases shared decision making in breast reconstruction. Annals of Surgical Oncology, 17, 738-743. doi: 10.1245/s10434-009-0869-7

Leerlooijer, J.N., Ruiter, R.A.C., Reinders, J., Darwisyah, W., Kok, G., \& Bartholomew, L.K. (2011). The World starts with me: using intervention mapping for the systematic adaptation and transfer of school-based sexuality education from Uganda to Indonesia. Translational Behavioral Medicine, 1, 331-340. doi:10.1007/s13142-011-0041-3

Lehmann, B.A., de Melker, H.E., Timmermans, D.R.M., \& Mollema, L. (2017). Informed decision making in the context of childhood immunization. Patient Education Counselling, 100(12), 2339-2345. doi: 10.1016/j.pec.2017.06.015

Lehmann, B.A., Ruiter, R.A.C., Wicker, S., Chapman, G., \& Kok, G. (2015). Medical students' attitude towards influenza vaccination. BMC Infectious Diseases, 15, 185. doi: 10.1186/s12879-015-09295

Lehtinen, M., Pavoonen, J., Wheeler, C.M., Jaisamram, U., Garland, S.M., Castellsagué, X., .... Dubin, G. (2012). Overall efficacy of HPV-16/18 AS04-adjuvanted vaccine against grade 3 or greater cervical intraepithelial neoplasia: 4-year end-of-study analysis of the randomised, double-blind PATRICIA trial. Lancet, 13(1), 89-99. doi: 10.1016/S1470-2045(11)70286-8

Liberman, A., \& Chaiken, S. (1992). Defensive processing of personally relevant health messages. Personality and Social Psychology Bulletin, 18(6), 669-679. doi: 10.1177/0146167292186002

Lichtenstein, S., Slovic, P., Fischhoff, B., Layman, M., \& Combs, B. (1978). Judged frequency of lethal events. Journal of Experimental Psychology: Human Learning and Memory, 4(6), 551-578. doi: 10.1037/0278-7393.4.6.551

Lin, M., Lucas, M.H.C, \& Shmueli, G. (2013). Too big to fail: large samples and the p-value problem. Information Systems Research, 24(4). 906-917. doi: 10.1287/isre.2013.0480

Lin, C.J., Nowalk, M.P., Toback, S.L., Rousculp, M.D., Raymund, M., Ambrose, C.S., \& Zimmerman, R.K. (2010). Importance of vaccination habit and vaccine choice on influenza vaccination among healthy working adults. Vaccine, 28(48), 7706-7712. doi : 10.1016/j.vaccine.2010.07.009

Loiselle, C.G., \& Dubois, S. (2009). The impact of a multimedia informational intervention on healthcare service use among women and men newly diagnosed with cancer. Cancer Nursing, 32, 37-44. doi :10.1097/01.NCC.0000343371.24517.66

Looijmans-van der Akker, I., van Delden, J.J., Verheij, T.J., van der Sande, M.A., van Essen, G.A., ..., \& Hak, E. (2010). Effects of a multi-faceted program to increase influenza vaccine uptake among health care workers in nursing homes: a cluster randomised control trial. Vaccine, 28, 5086-5092. doi : 10.1016/j.vaccine.2010.05.003

Lustria, M.L., Noar, S.M., Cortese, J., \& Van Stee, S.K., Glueckauf, R.L., \& Lee, J. (2013). A metaanalysis of web-delivered tailored health behavior change interventions. Journal of Health Communication, 18(9), 1039-1069. doi: 10.1080/10810730.2013.768727 
Maassen,G.H.,\&Bakker,A.B.(2001).Suppressorvariablesinpathmodels:Definitionsandinterpretations. Sociological Methods and Research, 30, 241-270. doi: 10.1177/0049124101030002004

Manfreda K.L., Bosnjak, M., Berzelak, J., Haas, I., \& Vehovar, V. (2008). Web surveys versus other survey modes: A meta-analysis comparing response rates. International Journal of Market Research, 50(1),79-104. doi: 10.1177/147078530805000107

Manhart, L.E., \& Koutsky, L.A. (2002). Do condoms prevent genital HPV infection, external genital warts, or cervical neoplasia? A meta-analysis. Sexually Transmitted Diseases, 29(11), 725-735.

Mao, J-Y., Vredenburg, K., Smith, P.W., \& Carey, T. (2005). The State of User-Centered Design Practice. Communications of the ACM, 48(3), 105-109. doi: 10.1145/1047671.1047677

Marteau, T.M., Dormandy, E., \& Michie, S. (2001). A measure of informed choice. Health Expectations, 4, 99-108. doi: 10.1046/j.1369-6513.2001.00140.x

McCredie, M.R., Sharples, K.J., Paul, C., Baranyai, J., Medley, G., Jones, R.W., \& Skegg, D.C. (2008). Natural history of cervical neoplasia and risk of invasive cancer in women with cervical intraepithelial neoplasia 3: A retrospective cohort study. Lancet Oncology, 9(5), 425-434. doi: 10.1016/S1470-2045(08)70103-7

McGuire, W.J. (1964). Inducing resistance to persuasion. In L. Berkowitz (Ed.), Advances in Experimental Social Psychology (Vol. 1, pp. 191-229). New York, NY: Academic Press.

Mennecke, B.E., Triplett, J.L., Hassall, L.M., Conde, Z.J., \& Heer, R. (2011). An Examination of a Theory of Embodied Social Presence in Virtual Worlds. Decision Science, 42, 413-450. doi: 10.1111/j.1540-5915.2011.00317.x

Mevissen, F.E.F., Meertens, R.M., \& Ruiter, R.A.C. (2012). Bedtime stories: The effects of selfconstructed risk scenarios on imaginability and perceived susceptibility to sexually transmitted infections. Psychology \& Health, 27, 1036-1047. doi: 10.1080/08870446.2011.648935

Mevissen, F.E.F., Meertens, R.M., Ruiter, R.A.C., Feenstra, H., \& Schaalma, H. (2009). HIV/STI Risk communication: The effects of scenario-based risk information and frequency-based risk information on perceived susceptibility to Chlamydia and HIV. Journal of Health Psychology, 14, 78-87. doi: 10.1177/1359105308097948

Mevissen, F.E.F., Ruiter, R.A.C., Meertens, R.M., \& Schaalma, H.P. (2010). The Effects of ScenarioBased Risk Information on Perceptions of Susceptibility to Chlamydia and HIV. Psychology \& Health, 25, 1161-1174. doi: 10.1080/08870440903055901

Michie, S., \& Abraham, C. (2008). Advancing the science of behaviour change: A plea for scientific reporting. Addiction, 103, 1409-1410. doi: 10.1111/j.1360-0443.2008.02291.x

Michie, S., Dormandy, E., \& Marteau, T.A. (2002). The multi-dimensional measure of informed choice: a validation study. Patient Education and Counseling, 48(1), 87-91.

Miller, W.R., \& Rollnick, S. (2002). Motivational interviewing: Preparing people for change (2nd ed.). New York: Guilford Press.

Miller, W.R., \& Rollnick, S. (2013). Motivational interviewing: Helping people change (3rd ed.). New York: Guilford Press. 
Mollers, M., Lubbers, K., Spoelstra, S.K., Weijmar-Schultz, W.C., Daemen, T., Westra, T.A., .... Tami, A. (2014). Equity in human papilloma virus vaccination uptake?: sexual behaviour, knowledge and demographics in a cross-sectional study in (un)vaccinated girls in the Netherlands. $B M C$ Public Health, 14, 288. doi: 10.1186/1471-2458-14-288

Mollen, S., Ruiter, R.A.C., \& Kok, G. (2010). Current issues and new directions in Psychology and Health: What are the oughts? The adverse effects of using social norms in health communication. Psychology and Health, 25(3), 265-270. doi: 10.1080/08870440903262812

Moore, G., Audrey, S., Barker, M., Bond, L., Bonell, C., Cooper, C., ... Baird, J. (2014).

Process evaluation in complex public health intervention studies: the need for guidance.

Journal of Epidemiology and Community Health, 68(2), 101-102.

doi: 10.1136/jech-2013202869

Moscicki, A.B., Schiffman, M., Burchell A, Albero, G., Giuliano, A.R., Goodman, M.T.,...

\& Palefsky, J. (2012). Updating the natural history of human papillomavirus and anogenital cancers. Vaccine, 30(5), F24-F33. doi : 10.1016/j.vaccine.2012.05.089

Morrison, L.G., Yardley, L., Powell, J., \& Michie, S. (2012). What design features are used in effective e-health interventions? A review using techniques from Critical Interpretive Synthesis. Telemedicine and e-Health, 18(2), 137-144. doi: 10.1089/tmj.2011.0062

Munoz, N., Bosch, F.X., Castellsague, X., Diaz, M., de Sanjose, S., Hammouda, D., ... Meijer, C. J., (2004). Against which human papillomavirus types shall we vaccinate and screen? The international perspective. International Journal of Cancer, 111, 278-285. doi: 10.1002/ijc.20244

Munoz, N., Castellsague, X., de Gonzalez, A.B., \& Gissmann, L. (2006). Chapter 1: HPV in the etiology of human cancer. Vaccine, 24(3): S1-S10. doi: 10.1016/j.vaccine.2006.05.115

Nan, X., Dahlstrom, M.F., Richards, A., \& Rangarajan, S. (2015). Influence of evidence type and narrative type on HPV risk perception and intention to obtain the HPV vaccine. Health Communication, 30, 301-308. doi: 10.1080/10410236.2014.888629

Neville, L.M., O’Hara, B., \& Milat, A.J. (2009). Computer-tailored dietary behaviour change interventions: a systematic review. Health Education Research, 24(4), 699-720. doi: 10.1093/her/ cyp006

Niccolai, L.M., \& Hansen, C.E. (2015). Practice- and Community-Based Interventions to Increase Human Papillomavirus Vaccine Coverage: A Systematic Review. JAMA Pediatrics, 169(7), 686692. doi: 10.1001/jamapediatrics.2015.0310.

Noar, S.M., Benac, C.N., \& Harris, M.S. (2007). Does tailoring matter? Meta-analytic review of tailored print health behavior change interventions. Psychology Bulletin, 133(4), 673-693. doi: 10.1037/0033-2909.133.4.673

Noar, S.M., \& Harrington, N. (2012). eHealth applications: Promising strategies for behavior change. New York: Routledge.

Noar, S.M., \& Willoughby, J.F. (2012). eHealth interventions for HIV prevention. AIDS Care, 24(8), 945-952. doi: 10.1080/09540121.2012.668167

O'Connor, A.M. (1995). Validation of a decisional conflict scale. Medical Decision Making, 15(1), 2530. doi: 10.1177/0272989X9501500105 
O'Connor, A.M., Jacobsen, M.J., \& Stacey D. (2002). An evidence-based approach to managing women's decisional conflict. Journal of Obstetric, Gynecologic, and Neonatal Nursing, 31, 570581.

Oenema, A., Brug, J., Dijkstra, A., de Weerdt, I., \& De Vries, H. (2008). Efficacy and use of an internetdelivered computer-tailored lifestyle intervention, targeting saturated fat intake, physical activity and smoking cessation: a randomized controlled trial. Annals of Behavioral Medicine, 35, 125 35. doi: $10.1007 / \mathrm{s} 12160-008-9023-1$

Oenema, A., Tan, F., \& Brug, J. (2005). Short-term efficacy of a Web-based computertailored nutrition intervention: main effects and mediators. Annals of Behavioral Medicine, 29, 54-63. doi: 10.1207/ s15324796abm2901_8

Okun, B.F. (2008). Effective Helping: Interviewing and Counseling Techniques (7th ed.). Belmont, CA: Thomson Brooks/Cole.

Palmer, P.B., \& O'Connell, D.G. (2009). Regression Analysis For Prediction: Understanding the process. Cardiopulmonary Physical Therapy Journal, 20(3), 23-26.

Paulussen, T.G.W., Hoekstra, F., Lanting, C.I., Buijs, G.B., \& Hirasing, R.A. (2006). Determinants of Dutch parents' decisions to vaccinate their child. Vaccine, 24(5), 644-651. doi: 10.1016/j. vaccine.2005.08.053

Paulussen, T.G., Lanting, C.I., Buijs, G.B., \& Hirasing, R.A. (2000). Parents about the governments immunization program: Satisfaction and vaccine intention of Dutch parents of toddlers. Leiden: TNO.

Peters, G-J.Y., de Bruin, M., \& Crutzen, R. (2013). Everything should be as simple as possible, but no simpler: towards a protocol for accumulating evidence regarding the active content of health behavior change interventions. Health Psychology Review, 9, 1-14. doi: 10.1080/17437199.2013.848409

Petty, R.E., Barden, J., \& Wheeler, S.C. (2009). The Elaboration Likelihood Model of Pursuasion: Developing health promotions for sustained behavioral change. In Diclimente, R.J., Crosby, R.A., \& Kegler M (Eds.). Emerging theories in health promotion practice and research (2nd ed., pp. 185-214). San Francisco: Jossey-Bass.

Petty, R.E., \& Cacioppo, J.T. (1986). The elaboration likelihood model of persuasion. In L. Berkowitz (Eds.), Advances in experimental social psychology (Vol. 19, pp. 123-205). Orlando, Florida: Academic Press.

Pot, M., Paulussen, T.G.W.M., Ruiter, R.A.C., Eekhout, I., de Melker, H.E., Spoelstra, M.E.A., \& van Keulen, H.M. (2017a). Effectiveness of a Web-based tailored intervention with virtual assistants promoting the acceptability of HPV vaccination among mothers of invited girls: a Randomized Controlled Trial. Journal of Medical Internet Research, 19(9), e312. doi: 10.2196/jmir.7449

Pot, M., Van Keulen, H.M., Ruiter, R.A.C., Eekhout, I., Mollema, L., \& Paulussen, T.W.G.M. (2017b). Motivational and contextual determinants of HPV vaccination uptake: A longitudinal study among mothers of girls invited for the HPV vaccination. Preventive Medicine, 100, 41-49. doi: 10.1016/j.ypmed.2017.04.005 
Priest, H.M., \& Knowlden, A.P. (2015). Systematic review of primary prevention human papillomavirus interventions targeting college students. International Journal of Sexual Health, 27, 125-144. do i:10.1080/19317611.2014.945631

Priester, J.R., \& Petty, R.E. (2003). The influence of spokesperson trustworthiness on message elaboration, attitude strength, and advertising effectiveness. Journal of Consumer Psychology, 13, 408-421. doi: 10.1207/S15327663JCP1304_08

Prochaska, J.O., Redding, C.A., \& Evers, K.E. (2015). The transtheoretical model of stages of change. In Glanz K, Rimer BK, Viswanath K (Eds), Health behavior: Theory, research and practice $\left(5^{\text {th }}\right.$ ed.). San Fransisco: Jossey-Bass.

Prochaska, J.O., Wright, J.A., \& Velicer, W.F. (2008). Evaluating Theories of Health Behavior Change: A hierarchy of Criteria Applied to the Transtheoretical Model. Applied Psychology, 57(4), 561588. doi:10.1111/j.1464-0597.2008.00345.x

Puthanakit, T., Schwartz, T. \& Esposito, S. (2013). Immune responses to a 2-dose schedule of the hpv$16 / 18$ as04-adjuvanted vaccine in girls (9-14) versus 3 doses in women (15-25): a randomised trial. in EUROGIN 2013.

R Development Core Team. (2009). R: A language and environment for statistical computing. Vienna: R Foundation for Statistical Computing.

Reiter, P.L., Brewer, N.T., Gottlieb, S.L., McRee, A.L., \& Smith, J.S. (2009). Parents' health beliefs and HPV vaccination of their adolescent daughters. Social Science and Medicine, 69(3), 475-480. doi: 10.1016/j.socscimed.2009.05.024

Resnicow, K., Braithwaite, R.L., Glanz, K., Rimer, B.K., \& Lewis, F.M. (2002). Applying theory to culturally diverse and unique populations. Health Behavior and Health Education; Theory, Research, and Practice ( $3^{\text {rd }}$ ed, pp. 485-509). San Francisco, CA: Jossey-Bass.

Riley, W.T., Rivera, D.E., Atienza, A.A., Nilsen, W., Allison, S.M., \& Mermelstein, R. (2011). Health behavior models in the age of mobile interventions: are our theories up to the task? Translational Behavioral Medicine, 1(1), 53-71. doi:10.1007/s13142-011-0021-7

Rondy, M., van Lier, A., van de Kassteele, J., Rust, L., \& de Melker, H. (2010). Determinants for HPV vaccine uptake in the Netherlands: a multilevel study. Vaccine, 28(9), 2070-2075. doi: 10.1016/j. vaccine.2009.12.042.

Rubin, D.B. (1987). Multiple Imputation for Nonresponse in Surveys. New York: Wiley.

Ruiter, R.A.C., Abraham, C., \& Kok, G. (2001). Scary warnings and rational precautions: A review of the psychology of fear appeals. Psychology and Health, 16, 613-630. doi: 10.1080/08870440108405863

Ruiter, R.A., Kessels, L.T., Jansma, B.M., \& Brug, J. (2006). Increased attention for computer-tailored health communications: an event-related potential study. Health Psychology, 25, 300-306. doi: 10.1037/0278-6133.25.3.300

Ruiter, R.A.C., Verplanken, B., De Cremer, D., \& Kok, G. (2004). Danger control and fear control in response to fear appeals: the role of need for cognition. Basic and Applied Social Psychology, 26, 13-24. doi: 10.1207/s15324834basp2601_2 
Russell, K.M., Champion, V.L., Monahan, P.O., Millon-Underwood, S., Zhao, Q., Spacey, N., ... Paskett, E.D. (2010). Randomized trial of a lay health advisor and computer intervention to increase mammography screening in African American women. Cancer Epidemiology, Biomarkers and Prevention, 19(1), 201-210. doi:10.1158/1055-9965.EPI-09-0569

Ryan, R.M., Lynch, M.F., Vansteenkiste, M., \& Deci, E.L. (2011). Motivation and autonomy in counseling, psychotherapy, and behavior change: a look at theory and practice. Counseling Psychology, 39, 193-260. doi: 10.1177/0011000009359313

Ryan, R.M., Patrick, H., Deci, E.L., \& Williams, G.C. (2008). Facilitating health behaviour change and its maintenance: Interventions based on Self-Determination Theory. European Health Psychology, 10(1), 2-5.

Schaalma, H., \& Kok, G. (2009). Decoding health education interventions: The times are a-changin. Psychology and Health, 24, 5-9. doi: 10.1080/08870440903126348

Schiffman, M., \& Castle, P.E. (2003). Human papillomavirus: epidemiology and public health. Archives of Pathology \& Laboratory Medicine, 127(8), 930-934. doi: 10.1043/1543 2165(2003)127<930:HP $\mathrm{EAPH}>2.0 . \mathrm{CO} ; 2$

Schiffman, M., Castle, P.E., Jeronimo, J., Rodriguez, A.C., \& Wacholder, S. (2007). Human papillomavirus and cervical cancer. Lancet, 370(9590), 890-907. doi: 10.1016/S01406736(07)61416-0

Scott, M., Pereira, L., \& Oakley, I. (2015). Show me or tell me: Designing avatars for feedback. Interacting with Computers, 27(4), 458-469. doi: 10.1093/iwc/iwu008

Schwarzer, R. (2001). Social-cognitive factors in changing health-related behavior. Current Directions in Psychological Science, 10, 47-51. doi: 10.1111/1467-8721.00112

Sheeran, P. (2002). Intention-behavior relations: A conceptual and empirical review. European review of social psychology, 12, 1-36. doi: 10.1080/14792772143000003

Shrout, P.E., \& Bolger, N. (2002). Mediation in experimental and nonexperimental studies: new procedures and recommendations. Psychological Methods, 7, 422-445. doi: 10.1037//1082989X.7.4.422

Singal, A.G., Higgins, P.D.R., \& Waljee, A.K. (2014). A primer on effectiveness and efficacy trials. Clinical and Translational Gastroenterology, 5(1), e45. doi: 10.1038/ctg.2013.13

Smulian, E.A., Mitchell, K.R., \& Stokley, S. (2016). Interventions to increase HPV vaccination coverage: a systematic review. Human Vaccines and Immunotherapeutics, 12, 1566-1588. doi: 10.1080/21645515.2015.1125055.

Spittaels, H., De Bourdeaudhuij, I., \& Vandelanotte, C. (2007). Evaluation of a websitedelivered computer-tailored intervention for increasing physical activity in the general population. Preventive Medicine, 44(3), 209-217. doi: 10.1016/j.ypmed.2006.11.010

Steen, R.G. (2007). The evolving brain: The known and the unknown. Amherst, NY: Prometheus Books. Tversky, A., \& Kahneman, D. (1974). Judgment under uncertainty: Heuristics and biases. Science, 185, 1124-1131. doi: 10.1126/science.185.4157.1124

Twisk J. (2006). Applied Multilevel Analysis. Cambridge: Cambridge University Press. 
Van Breukelen, G.J. (2006). ANCOVA versus change from baseline: More power in randomized studies, more bias in nonrandomized studies. Journal of Clinical Epidemiology, 59, 920-925. doi: 10.1016/j.jclinepi.2006.02.007

Van Buuren, S. (2012). Flexible Imputation of Missing Data. Boca Raton, FL: CRC Press.

Van der Pal, S., Otten, W., \& Detmar, S. (2010). Evaluatie van de voorlichting aan ouders over de hielprik. Tijdschrift voor gezondheidswetenschappen, 88, 449-453.

Van Kesteren, N.M.C., Kok, G., Hospers, H.J., Schippers, J., \& De Wildt, W. (2006). Systematic development of a self-help and motivational enhancement intervention to promote sexual health in hiv-positive men who have sex with men. AIDS Patient Care STDS, 20, 858-875. doi: 10.1089/apc.2006.20.858

Van Keulen, H.M., Otten, W., Ruiter, R.A.C., Fekkes, M., Van Steenbergen, J., Dusseldorp, E., \& Paulussen, T.W.G.M. (2013a). Determinants of HPV vaccination intentions among Dutch girls and their mothers: A cross-sectional study. BMC Public Health, 13, 111. doi: 10.1186/14712458-13-111

Van Keulen, H.M., Otten, W., Ruiter, R.A.C., Van Steenbergen, J., Fekkes, M., \& Paulussen, T.W.G.M. (2013b). Redenen om zich te laten vaccineren tegen HPV. Nederlandse Tijdschrift voor Geneeskunde, 157, A5523.

Van Keulen, H.M., Fekkes, M., Otten, W., Van der Pal, S., Kocken, P., Ruiter, R.A.C., \& Paulussen, T.G.W.M. (2010). Onderzoek naar de HPV-vaccinatiebereidheid bij moeders en dochters naar aanleiding van de inhaalcampagne in Nederland. Report KvL/GB.

van Lier E.A., Geraedts, J.L.E., Oomen, P.J., Giesbers, H., van Vliet, J.A., Drijfhout IH,...de Melker, H.E. (2017). Vaccinatiegraad en jaarverslag Rijksvaccinatieprogramma Nederland 2016. Retrieved from: https://www.rivm.nl/dsresource?objectid=2807c63f-3223-4f2e-b488-7e3126b 03072\&type $=$ pdf\&disposition $=$ inline

van Lier E.A., Geraedts, J.L.E., Oomen, P.J., Giesbers, H., van Vliet, J.A., Drijfhout IH,...de Melker, H.E. (2018). Vaccinatiegraad en jaarverslag Rijksvaccinatieprogramma Nederland 2017. Retrieved from: https://www.rivm.nl/dsresource?objectid=30c7c6ab-197d-44a0-a901-f9719f916bf9\&type $=$ pdf\&disposition $=$ inline

Van Lier, E.A., Oomen, P.J., de Hoogh, P., Drijfhout, I., Elsinghorst, B., Kemmeren, J., ..., de Melker, H.E. (2012). Praeventis, the immunization register of the Netherlands: a tool to evaluate the National Immunization Programme. Euro Surveillance, 17(17), pii=20153. doi: 10.2807/ ese.17.17.20153-en

Van Lier, E.A., Oomen, P.J., Giesbers, H., van Vliet, J.A., Drijfhout, I.H., Zonnenberg-Hoff, I.F., \& de Melker, H.E. (2016). Vaccinatiegraad Rijksvaccinatieprogramma Nederland: verslagjaar 2016. Retrieved from: http://www.rivm.nl/Documenten_en_publicaties/Wetenschappelijk/ Rapporten/2016/juni/Vaccinatiegraad_Rijksvaccinatieprogramma_Nederland_ Verslagjaar_2016

Van 't Riet, J., Cox, A.D., Cox, D., Zimet, G.D., De Bruijn, G.J., Van den Putte, B., ... Ruiter, R.A.C. (2014). Does perceived risk influence the effects of message framing? A new investigation of a widely held notion. Psychology and Health, 29(8), 37-41. doi:10.1080/08870446.2014.896916 
Van't Riet J., Crutzen R., \& De Vries, H. (2010). Investigating predictors of visiting, using and revisiting an online health-education programme: a longitudinal study. Journal of Medical Internet Research, 12, e37. doi: 10.2196/jmir.1345

Van't Riet, J., \& Ruiter, R.A.C. (2013). Defensive reactions to health-promoting information: An overview and implications for future research. Health Psychology Review, 7(1), S104-S136. doi: $10.1080 / 17437199.2011 .606782$

Van Vugt, H.C. (2008). Embodied Agents from a User's Perspective. Doctoral dissertation. VU Amsterdam: The Netherlands.

Verplanken, B., \& Aarts, H. (1999). Habit, attitude, and planned behavior: Is habit an empty construct or an interesting case of goal-directed automaticity? In W. Stroebe \& M. Hewstone (Eds.), European review of social psychology (Vol. 10, pp. 101-134). Chichester, England: Wiley.

Verplanken, B., \& Orbell, S. (2003). Reflections on past behavior: A self-report index of habit strength. Journal of Applied Social Psychology, 33, 1313-1330. doi: 10.1111/j.1559-1816.2003.tb01951.x

Wakeham, K., \& Kavanagh, K. (2014). The Burden of HPV-Associated Anogenital Cancers. Current Oncology Reports, 16, 402. doi: 10.1007/s11912-014-0402-4

Walling, E.B., Benzoni, N., Dornfeld, J., Bhandari, R., Sisk, B.A., Garbutt, J., \& Colditz, G. (2016). Interventions to improve HPV vaccine uptake: a systematic review. Pediatrics, 138(1), e20153863. doi: 10.1542/peds.2015-3863.

Walboomers, J.M., Jacobs, M.V., Manos, M.M., Bosch, F.X., Kummer, J.A., Shah, K.V., ... Munoz, N. (1999). Human papillomavirus is a necessary cause of invasive cervical cancer worldwide. Journal of Pathology, 189, 12-19. doi: 10.1002/(SICI)1096-9896(199909)189:1<12::AIDPATH431>3.0.CO;2-F

Watson, A., Bickmore, T., Cange, A., Kulshreshtha, A., \& Kvedar, J. (2012). An internet-based virtual coach to promote physical activity adherence in overweight adults: randomized controlled trial. Journal of Medical Internet Research, 14, e1. doi: 10.2196/jmir.1629

Webb, T.L., Joseph, J., Yardley, L., \& Michie, S. (2010). Using the internet to promote health behavior change: a systematic review and meta-analysis of the impact of theoretical basis, use of behavior change techniques, and mode of delivery on efficacy. Journal of Medical Internet Research, 12(1), e4. doi: 10.2196/jmir.1376

Weinberg, R.A. (1999). One renegade cell: how cancer begins ( $1^{\text {st }}$ ed.). New York: Basic Books.

White, I.R., Royston, P., \& Wood, A.M. (2011). Multiple imputation using chained equations: Issues and guidance for practice. Statistics in Medicine, 30, 377-399. doi: 10.1002/sim.4067

Whittingham, J.R., Ruiter, R.A.C., Castermans, D., Huiberts, A., \& Kok, G. (2008a). Designing effective health education materials: experimental pre-testing of a theory-based brochure to increase knowledge. Health Education Research, 23, 414-426. doi: 10.1093/her/cym018

Whittingham, J.R., Ruiter, R.A.C., Zimbile, F., \& Kok, G. (2008b). Experimental pretesting of public health campaigns: A case study. Journal of Health Communication, 13, 216-229. doi: $10.1080 / 10810730701854045$

Wik, P., \& Hjalmarsson, A. (2009). Embodied conversational agents in computer assisted language learning. Speech Communication, 51, 1024-1037. doi:10.1016/j.specom.2009.05.006 
Winer, R.L., Feng, Q., Hughes, J.P., O’Reilly, S., Kiviat, N.B., \& Koutsky, L.A. (2008). Risk of female human pappilomavirus acquisition associated with first male sax partner. Journal of Infectious Diseases, 197, 279-282. doi: 10.1086/524875

Winer, R.L., Hughes, J.P., Feng, Q., Xi, L.F., Cherne, S., O’Reilly, S., ... Koutsky, L.A. (2011). Early natural history of incident, type-specific human papillomavirus infections in newly sexually active young women. Cancer Epidemiology, Biomarkers, and Prevention, 20, 699-707. doi: 10.1158/1055-9965.EPI-10-1108

Winterbottom, A., Bekker, H., Conner, M., \& Mooney, A. (2008). Does narrative information bias individual's decision making? A systematic review. Social Science and Medicine, 67, 2079-2088. doi: 0.1016/j.socscimed.2008.09.037

Woodman, C. B., Collins, S., Winter, H., Bailey, A., Ellis, J., Prior, P., ... Young, L. S., (2001). Natural history of cervical human papillomavirus infection in young women: a longitudinal cohort study. Lancet, 357, 1831-1836. doi: 10.1016/S0140-6736(00)04956-4

Wright, P. (2011). Using graphics effectively in text. In C. Abraham \& M. Kools (Eds.), Writing health communication: An evidence-based guide. London: Sage Publications.

Wyer, R S. (1974). Cognitive organization and change: An information-processing approach (pp. 223). Hillsdale, NJ: Lawrence Erlbaum Associates. 


\section{APPENDIX A}

The text messages that were used in the experimental pretest.

\section{General information}

\section{HPV and cervical cancer}

Cervical cancer is a life-threatening disease among women. It is caused by the Human Pappilomavirus (HPV). HPV is contagious and is spread through sex: through contact with infected genital skin, mucous membranes, or bodily fluids. Both men and women can get infected with HPV. Not only the penis and vagina are infected; HPV can infect skin not normally covered by a condom (such as hands or mouth), so using a condom does not fully protect someone from the virus. Also, many people don't realize they're infected with HPV and may have no symptoms. The virus can be cleared by one's immune system, but sometimes, this does not happen. Then, the virus can lead to precursors of cervical cancer -and eventually to cervical cancer.

\section{The HPV vaccination}

Since 1976, there has been a cervical cancer screening program. Thanks to this program, precursors of cervical cancer can be detected early, so that cervical cancer can be prevented or treated at an early stage of the disease. In order to prevent (rather than detect) cervical cancer, the HPV vaccination has been available since 2010. Every year, 12 year old girls are invited via the National Immunization Program. The HPV vaccination consists of three injections ${ }^{*}$, given in a period of half a year. The HPV vaccination is offered on a voluntary basis and free of charge. The HPV vaccination prevents infection with the HPV-virus (2 types of the virus, that cause $70 \%$ of infections) and protects against cervical cancer. The $H P V$ vaccination is also called 'the vaccination against cervical cancer'. The HPV vaccination has been approved for girls between 9 and 26 years old, but it is best to vaccinate girls at an early age, before she gets sexually active and therefore is at risk for getting infected. The $\mathrm{HPV}$ vaccination has been tested and results showed it was safe. As with most vaccinations, the spot of injection can be a bit painful, red and swollen for a while.

There is no treatment if you are infected with HPV. The HPV vaccination is therefore the only available and effective method to protect against an infection with HPV

Note: The original language of the narrative information was Dutch. ${ }^{\star}$ When the experiment was conducted, the HPV vaccination still consisted of three instead of two injections. 


\section{Statistical risk information}

\section{The risk of getting HPV and cervical cancer}

In the Netherlands, every year, about 600 Dutch women are diagnosed with cervical cancer. Approximately 200 of these women die of this disease per year. This means that almost every day, a woman dies of cervical cancer. Cervical cancer is caused by infection with the $\mathrm{HPV}$-virus. The HPV-virus is highly prevalent. Almost everybody who is sexually active, is exposed to the virus. Of every 100 women, 80 get infected with the HPV-virus. This means that $80 \%$ of all women get infected with HPV at least once. However, not all women get cervical cancer. It cannot be predicted who gets cervical cancer and who doesn't, but it is always caused by being infected with HPV. Therefore, it is important to prevent a HPVinfection.

\section{Protecting against $\mathrm{HPV}$}

It is expected that, in the Netherlands, the HPV vaccination will prevent half of the cervical cancer cases. This means that the HPV vaccination can decrease the amount of women getting cervical cancer with $50 \%$. So, when no girl gets the HPV vaccination, 600 women per year will get cervical cancer. But when all girls get the HPV vaccination, 300 women per year (half of 600) will be saved from getting cervical cancer.

In conclusion: girls who dó get the HPV vaccination, have a much smaller chance of getting cervical cancer later in life than girls wo dón't get the HPV vaccination.

Note: The original language of the statistical information was Dutch.

\section{Narrative risk information}

By Anne, 30 years of age

Hi everybody. I found this forum via a friend and I would like to share my story with you all. When I was 14 years old, I had my first boyfriend, with whom I had sex for the first time when I was 16. Since then, I have had two relationships: one when I was 21 (lasted 2 years) and my current relationship with Robbert (already lasting 4 years now). I've only had a few casual sex partners in my life; I never was that 'wild'. Aside from a drunk slip-up, I've always used a condom with casual partners. I wouldn't want to get an STI!

Last year, we went skiing with a couple of friends. Just after our arrival, I noticed that I lost some blood. Somewhat concerned, I called a family member who also is a doctor. 'Could it be that you're pregnant?' he asked me. Robbert and I agreed that we were ready having a baby, so it was possible. We got the advice to enjoy our vacation and that I should have it checked out when I was back home. Once I was back home, I got my period and because 
we immediately were sucked into our busy but carefree lives, I kind of already had forgotten about what happened during our vacation. Until, about a month ago, I again had some blood loss while it wasn't the time to have my period. I arranged an appointment with my doctor that afternoon. After a short examination, I was sent to the hospital for further tests.

Turned out.. I have cervical cancer! The doctor told me that the cancer had been caused by the HPV-virus. I've had heard of it, but didn't know much about it. But I had been so careful? Could it be one of those very few times I have had unsafe sex? Could have been, but the doctor told me that I could have gotten infected with HPV during safe sex as well. It therefore was quite difficult to find out who got me infected. I'd never thought that I would get infected with HPV, let alone that it would develop into cervical cancer..

With the HPV vaccination that is available now, I sometimes can't help but wonder: 'what if I had gotten myself vaccinated against HPV 18 years ago...'

Note: The original language of the narrative information was Dutch. 


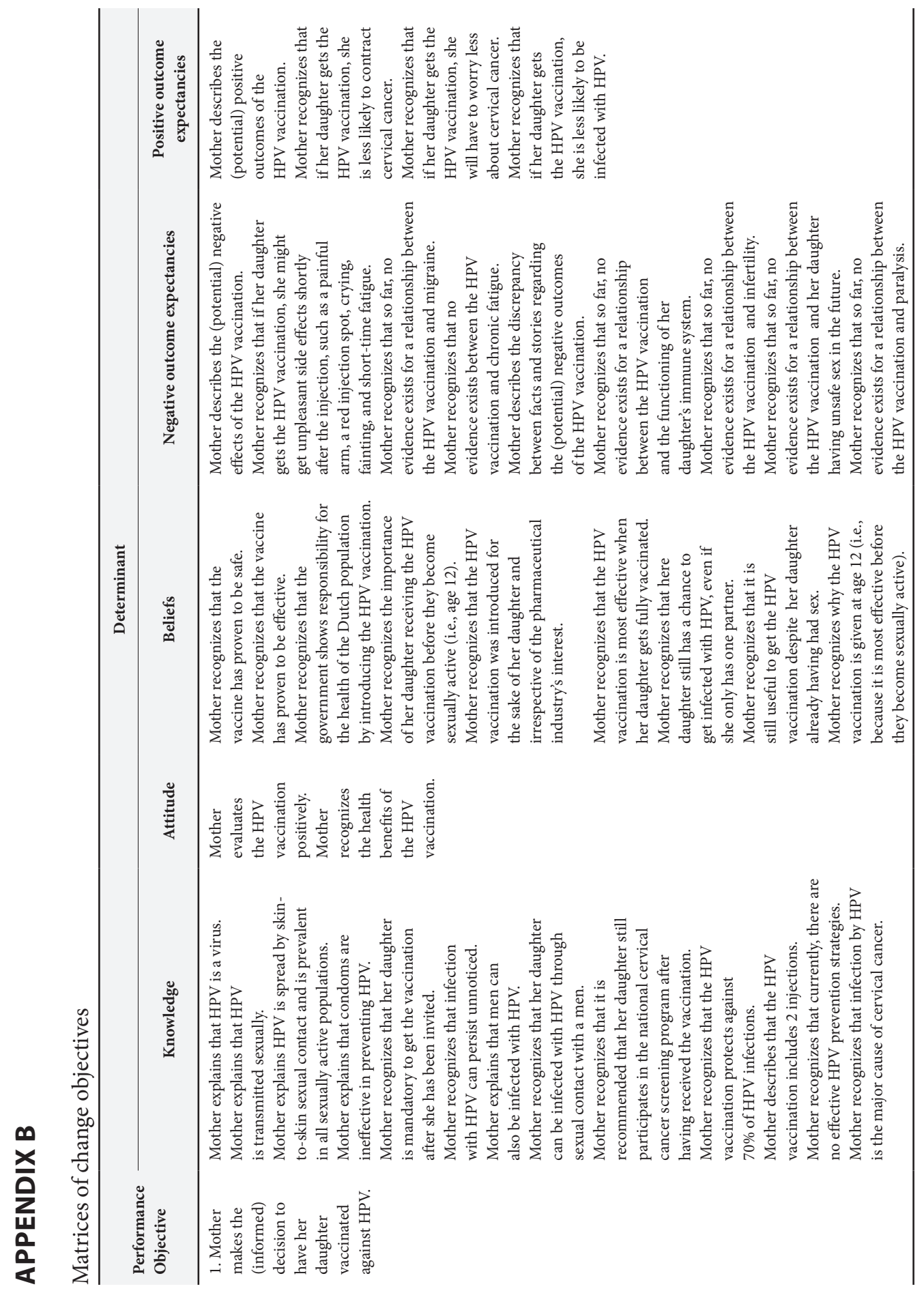




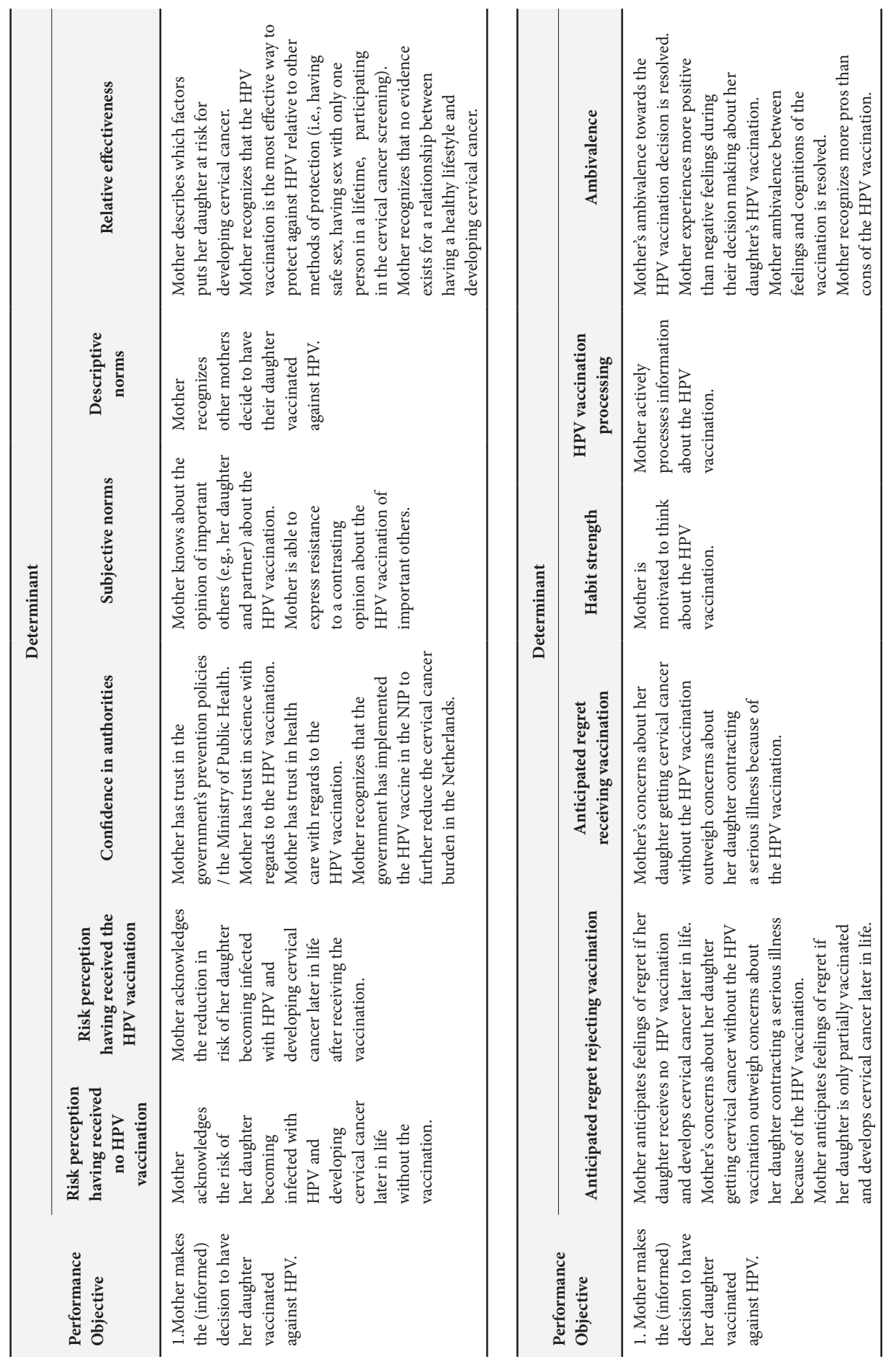




\begin{tabular}{lll}
\hline \multirow{2}{*}{ Performance Objective } & \multicolumn{2}{c}{ Determinant } \\
\cline { 2 - 3 } & \multicolumn{1}{c}{ Attitude } & Self-efficacy \\
\hline $\begin{array}{l}\text { 2. Mother discusses her decision } \\
\text { to have her daughter vaccinated } \\
\text { against HPV with her daughter } \\
\text { and important others. }\end{array}$ & $\begin{array}{l}\text { Mother evaluates communication } \\
\text { with important others positively. }\end{array}$ & $\begin{array}{l}\text { Mother expresses confidence in } \\
\text { discussing the decision to have } \\
\text { her daughter vaccinated against } \\
\text { HPV with important others. }\end{array}$ \\
\hline
\end{tabular}

\begin{tabular}{|c|c|c|}
\hline \multirow{2}{*}{ Performance Objective } & \multicolumn{2}{|c|}{ Determinant } \\
\hline & Knowledge & Beliefs \\
\hline $\begin{array}{l}\text { 3. Mother guides her daughter towards } \\
\text { receiving the first HPV injection. } \\
\text { 4. Mother guides her daughter towards } \\
\text { receiving both HPV injections. }\end{array}$ & $\begin{array}{l}\text { Mother knows where to get } \\
\text { the first HPV injection. } \\
\text { Mother describes that the second } \\
\text { HPV injection has to be received } 6 \\
\text { months after the first HPV injection. } \\
\text { Mother knows where to get } \\
\text { the second HPV injection. }\end{array}$ & $\begin{array}{l}\text { Mother recognizes that the HPV } \\
\text { vaccination is most effective when } \\
\text { her daughter gets fully vaccinated. }\end{array}$ \\
\hline
\end{tabular}




\section{APPENDIX C}

Screenshots of the four menus of the website.

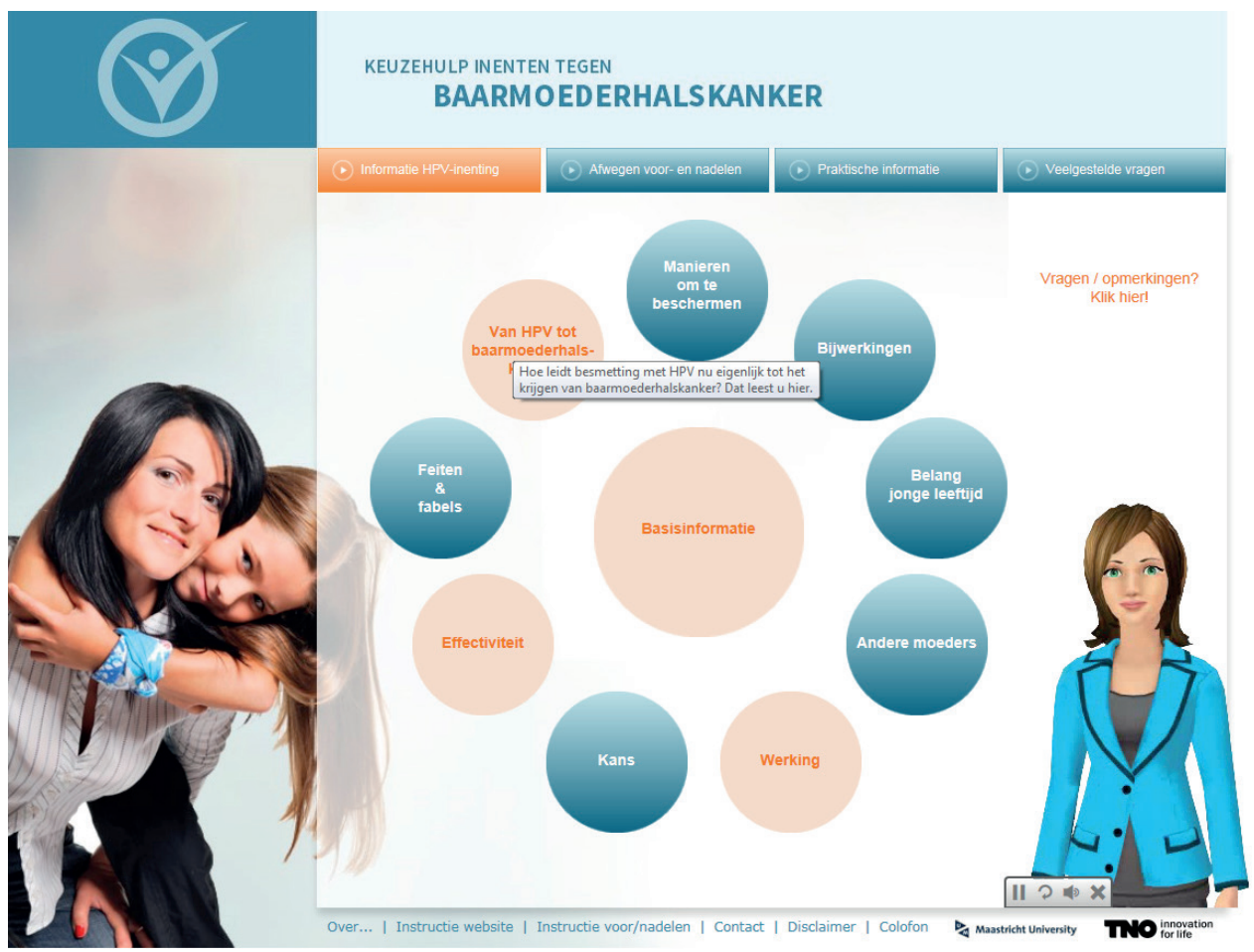

Picture 1: The first menu of the website ('information about the HPV vaccination'). 


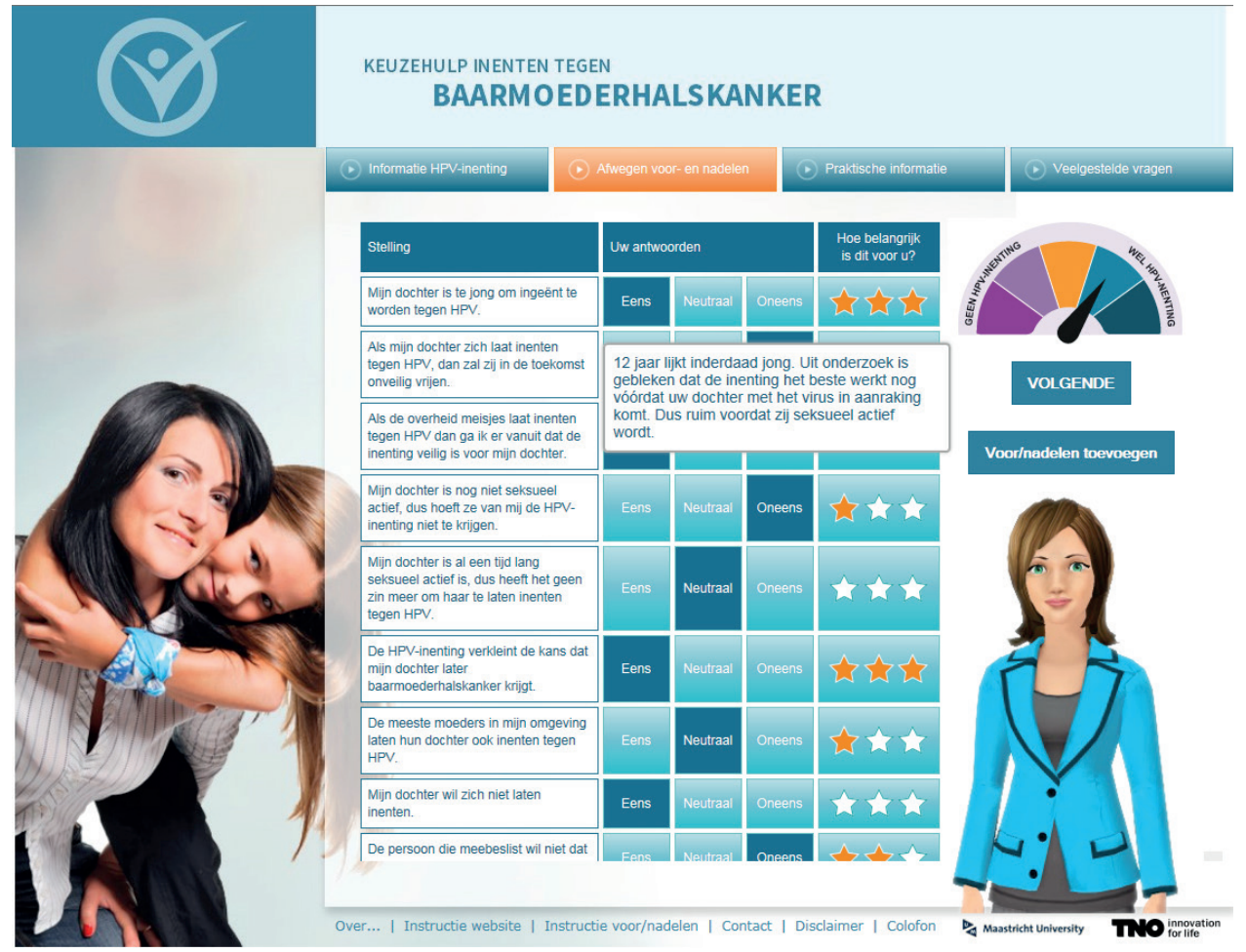

Picture 2: The second menu of the website ('weighing up the pros against the cons'). 


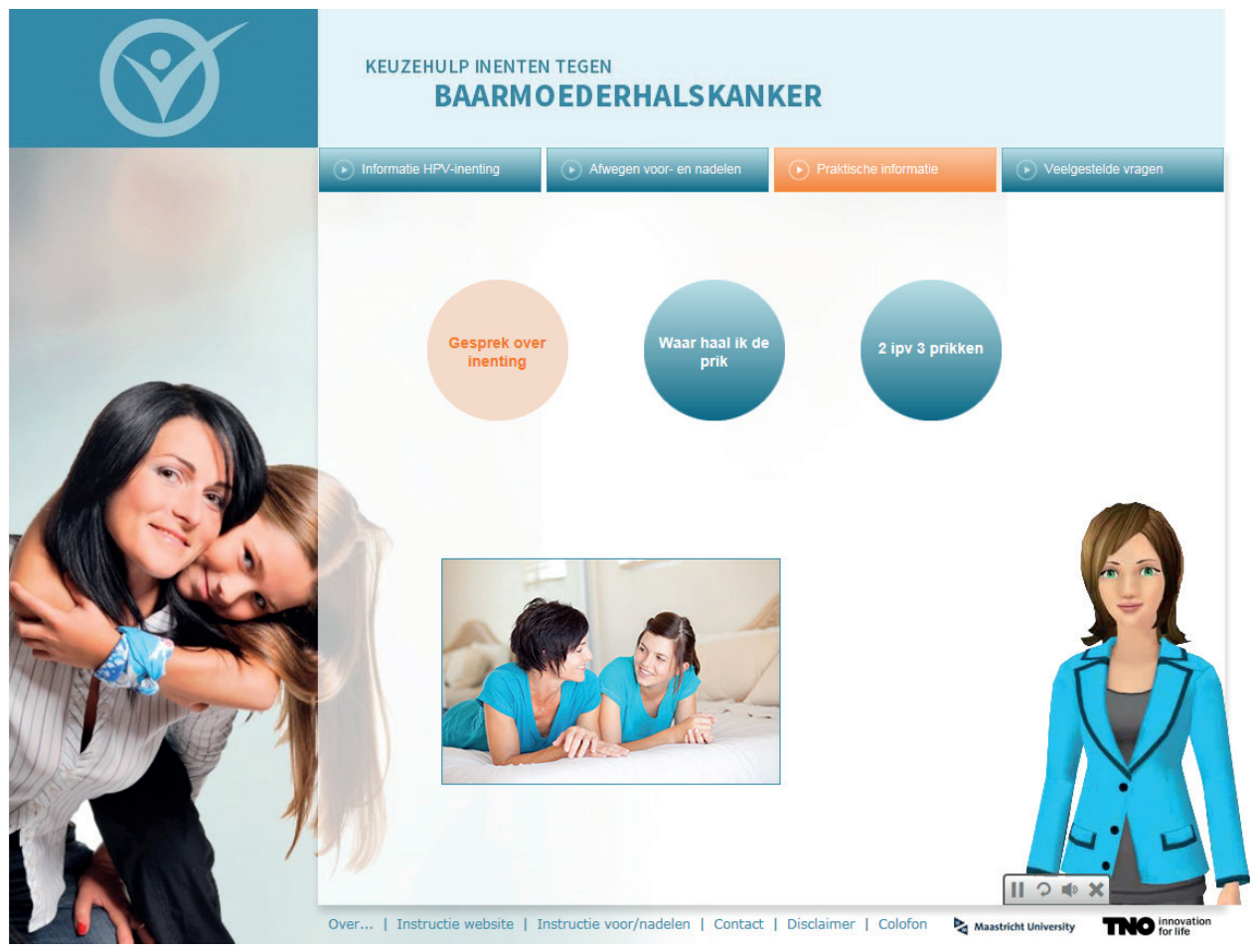

Picture 3: The third menu of the website ('practical information'). 


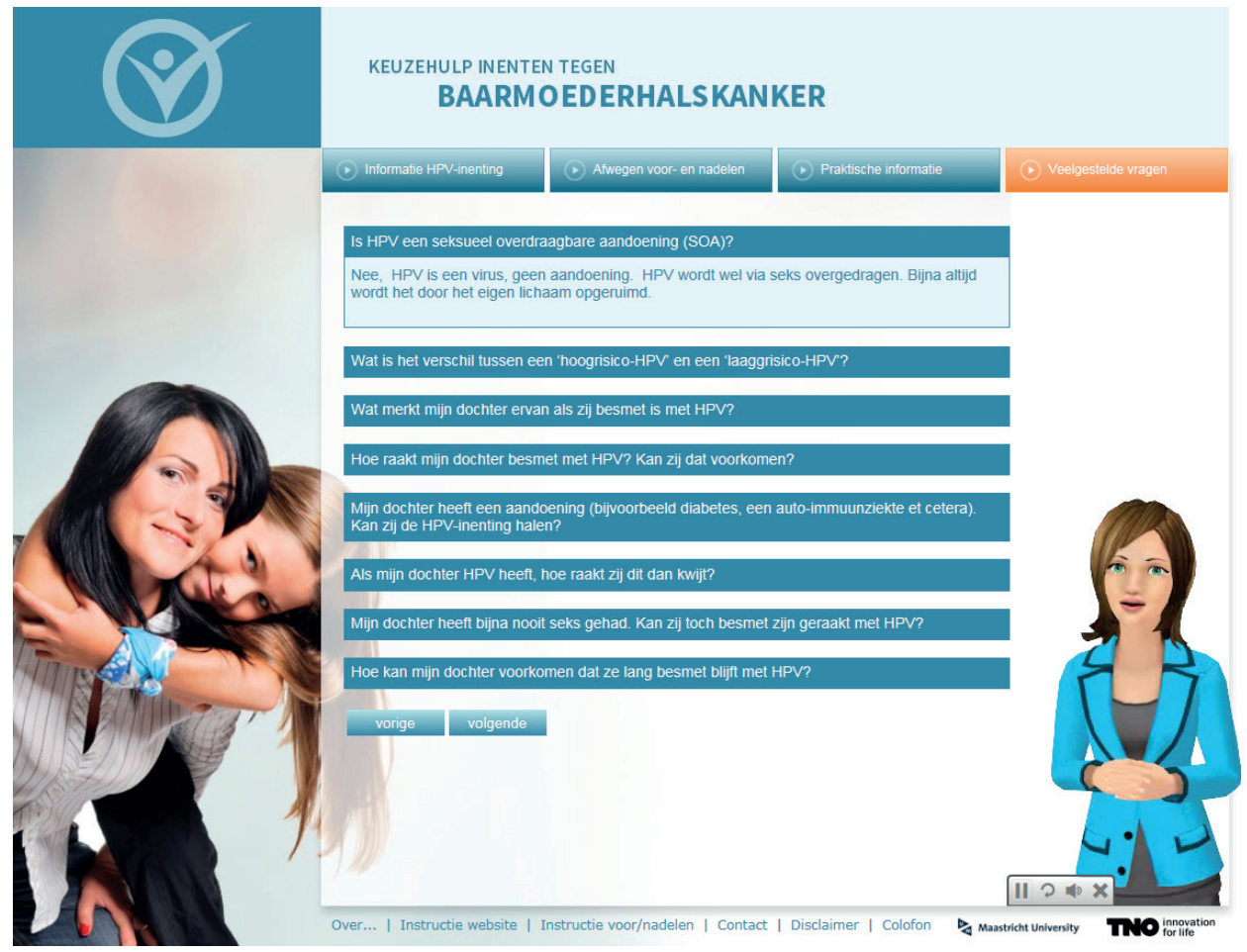

Picture 4: The fourth menu of the website ('frequently asked questions'). 


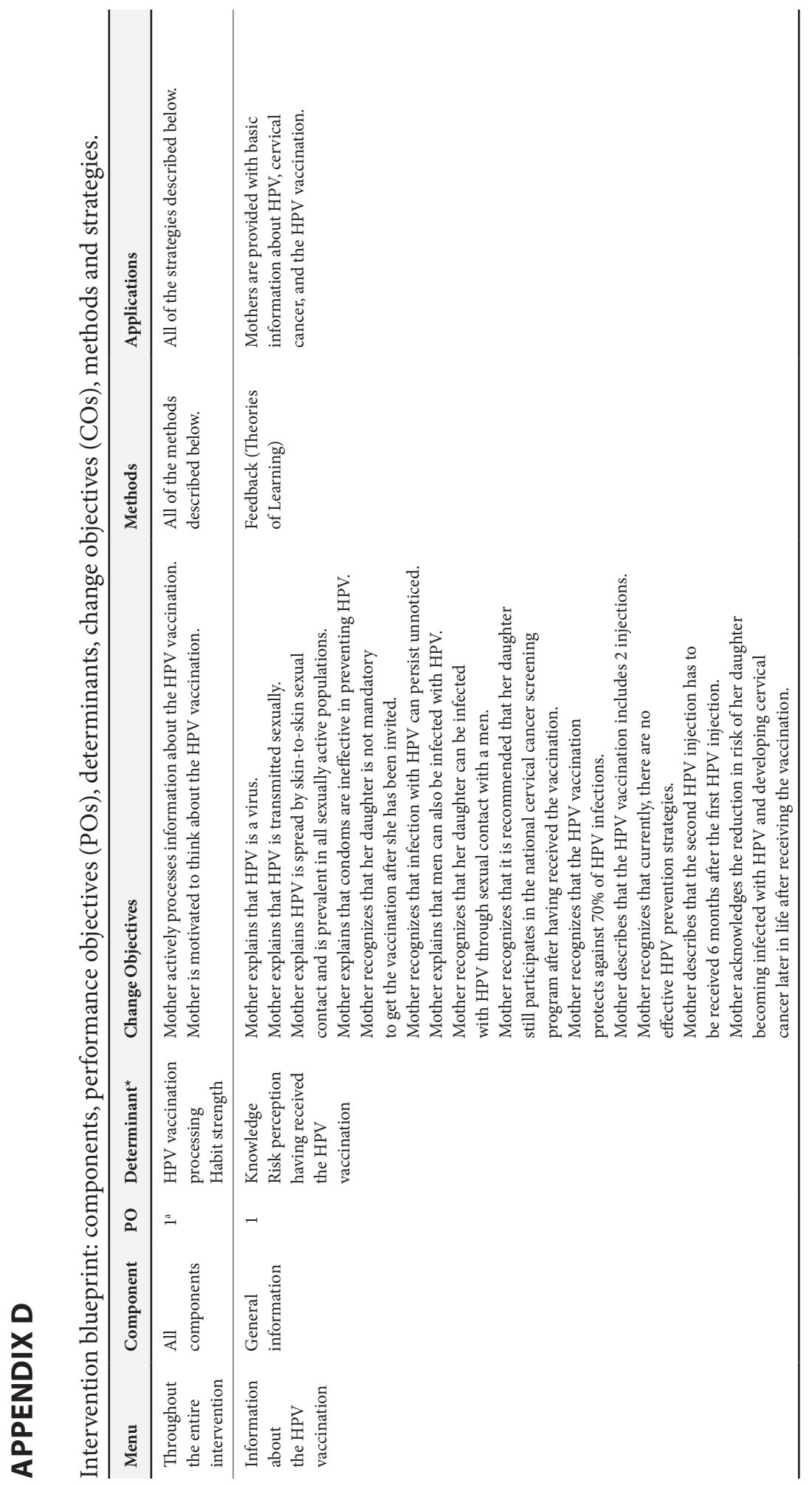




\section{Chapter 7}

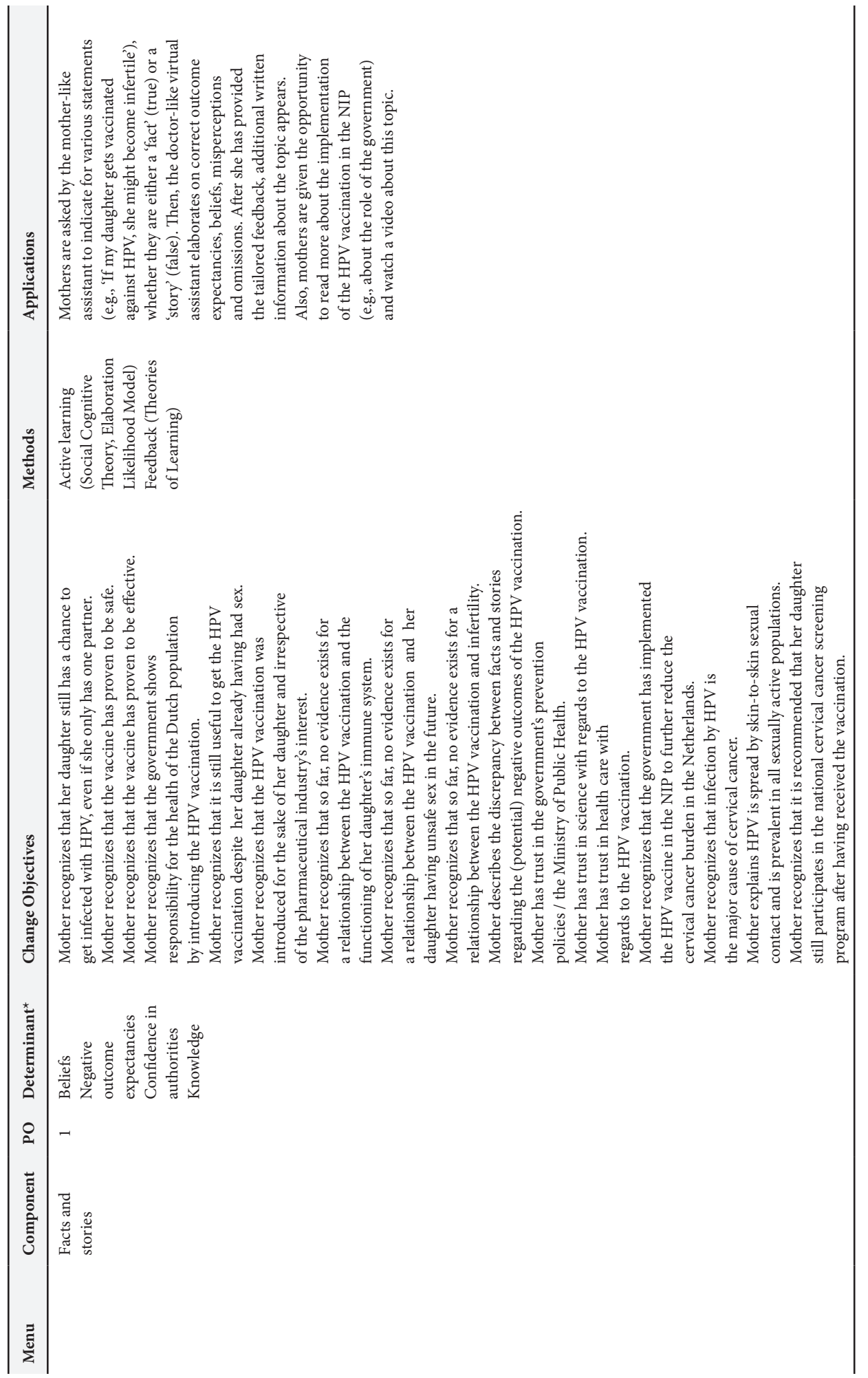




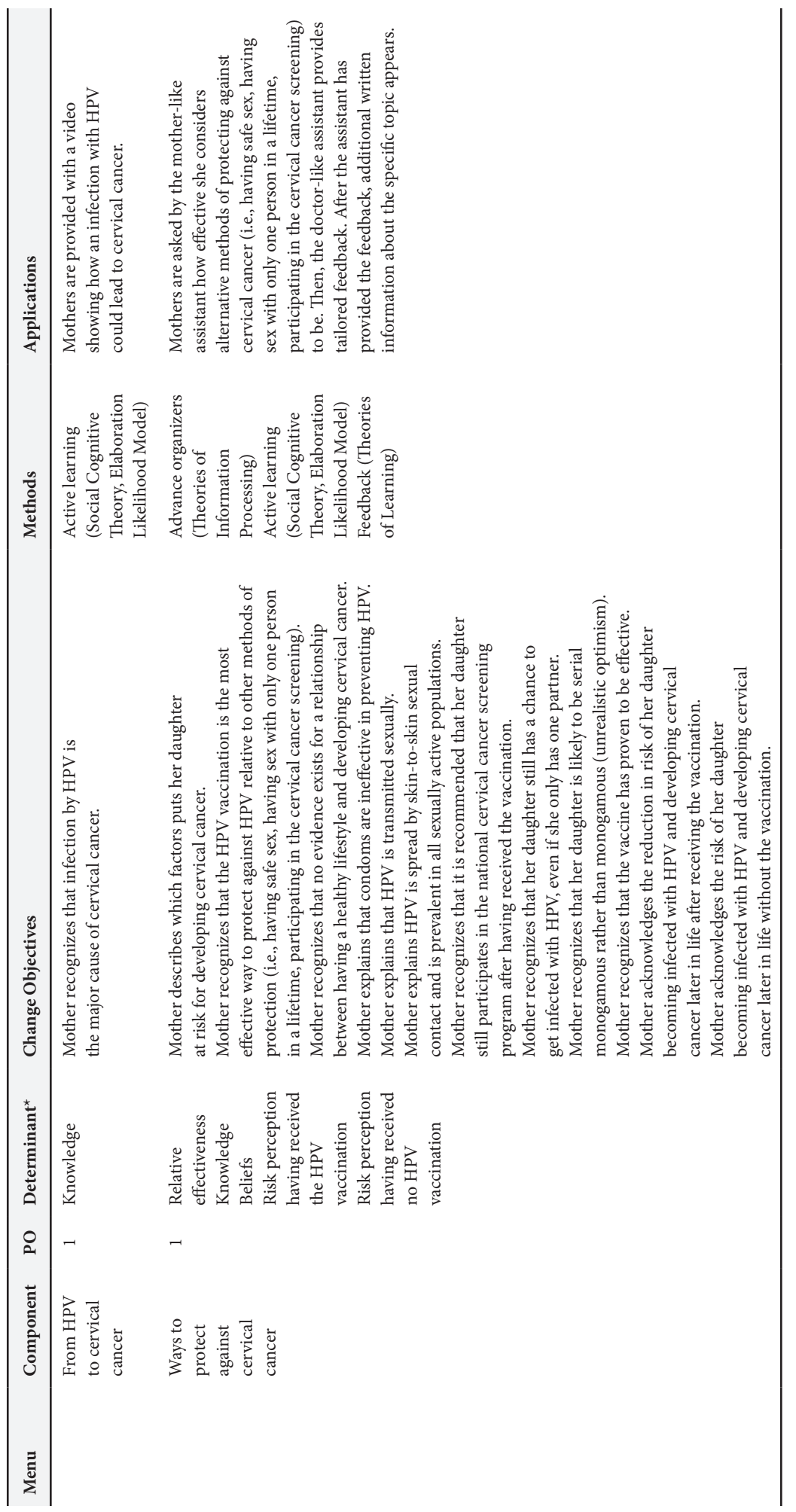




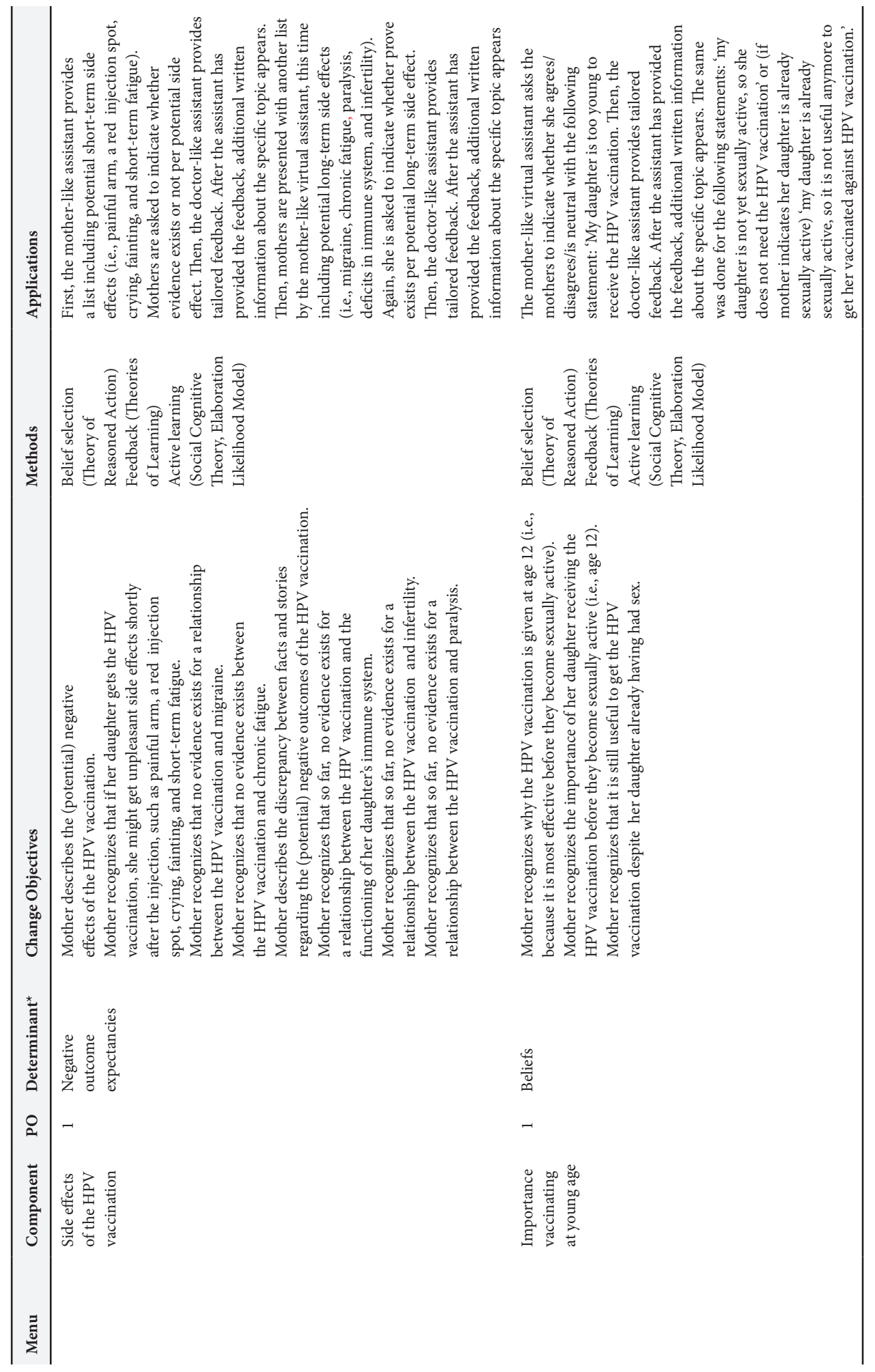




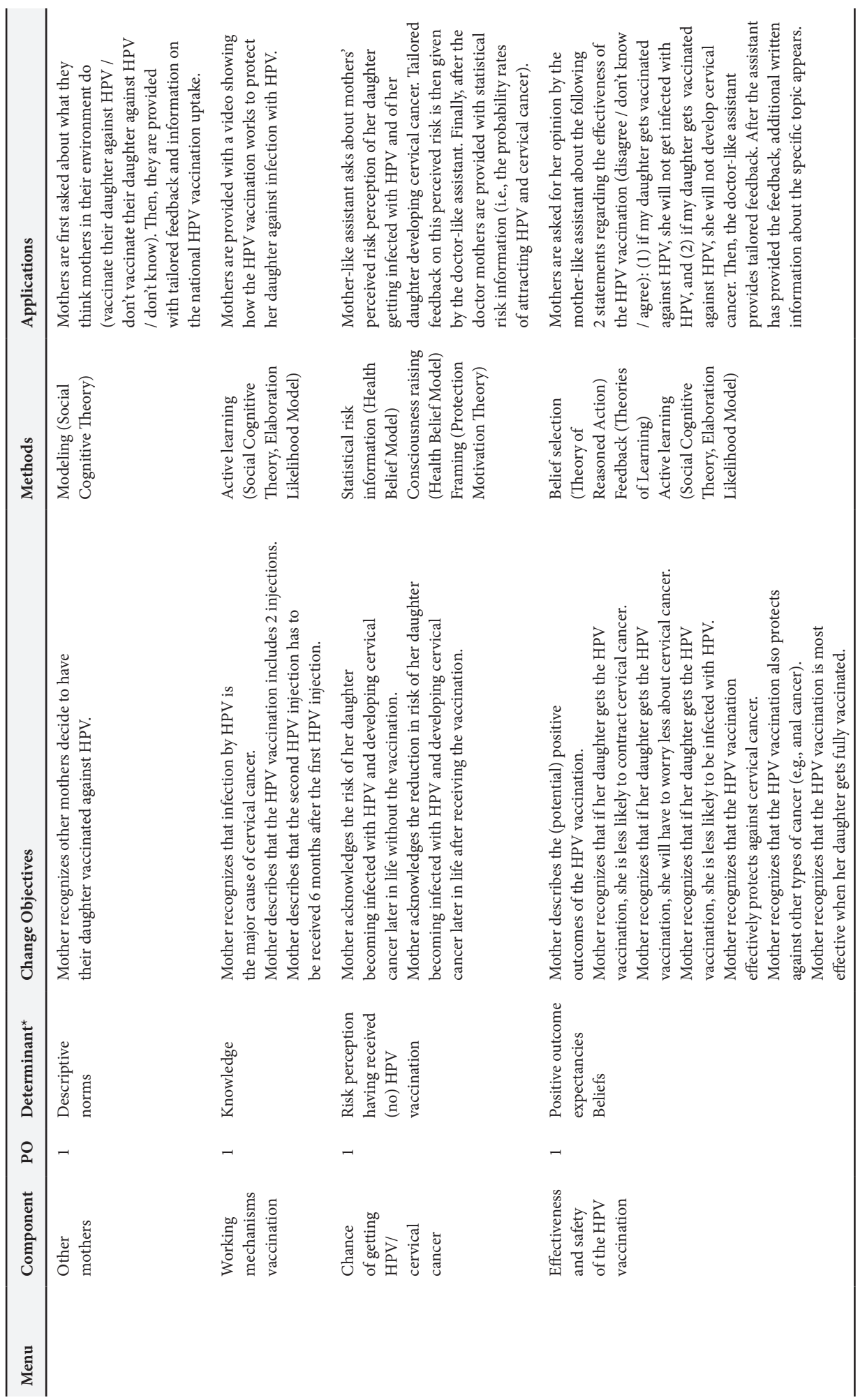




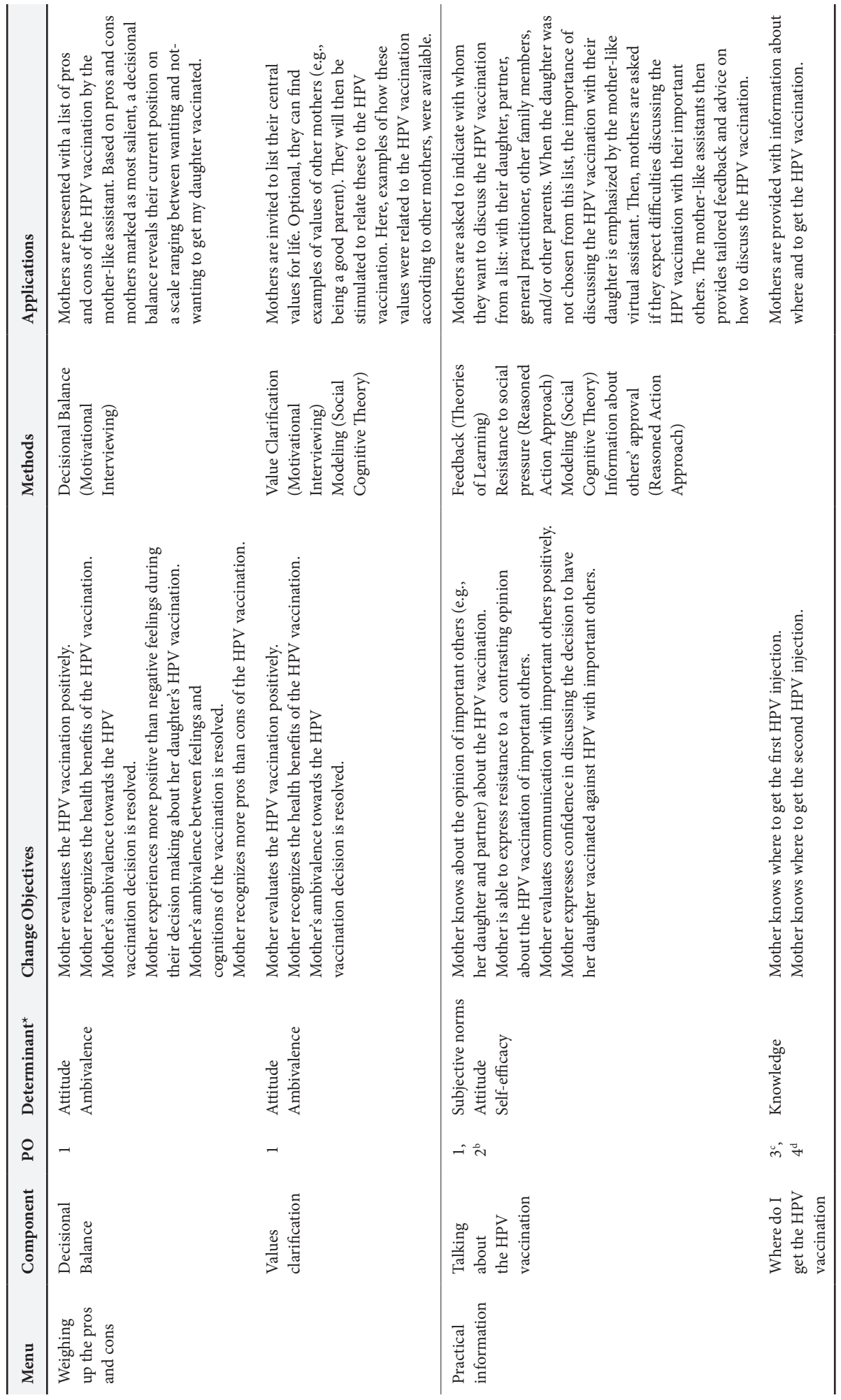




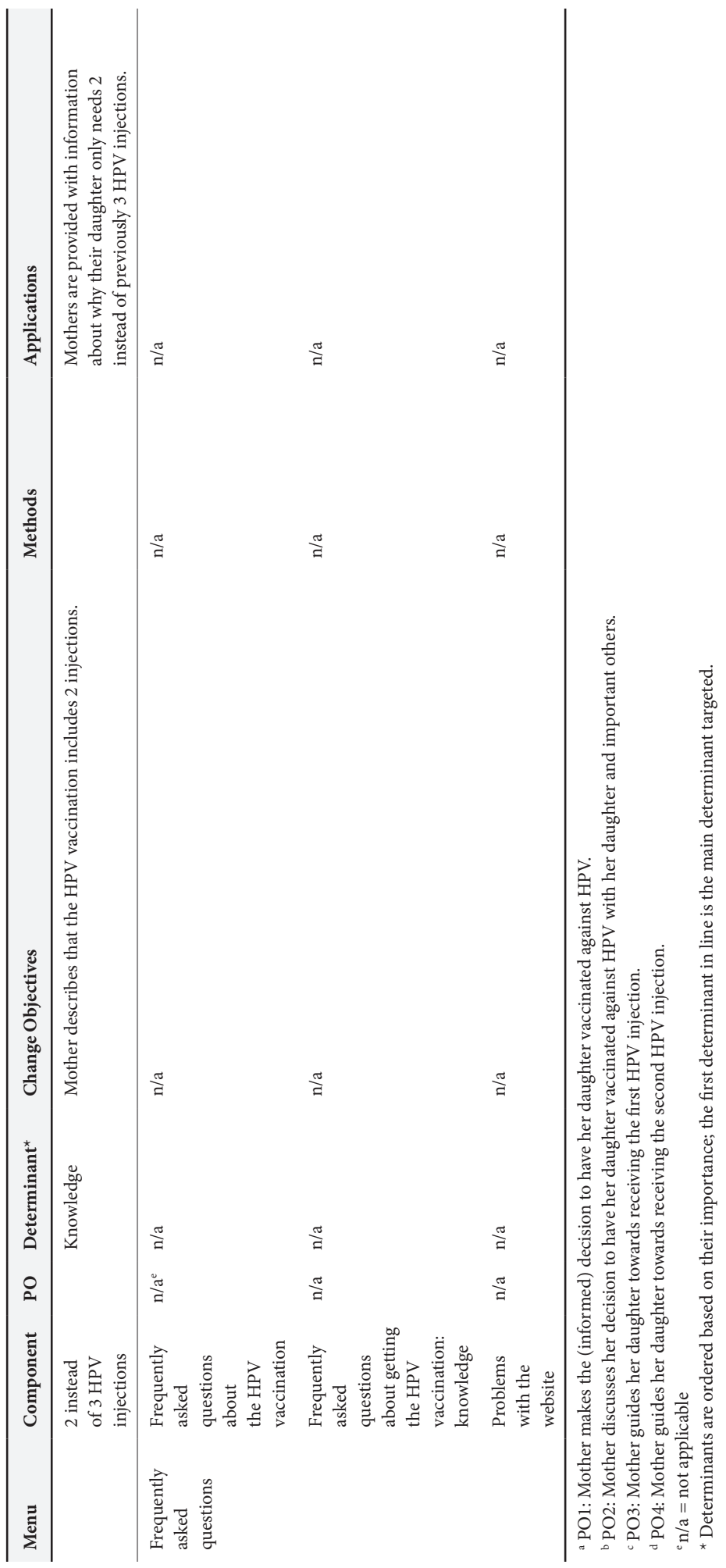




\section{APPENDIX E}

Screenshots of the website.

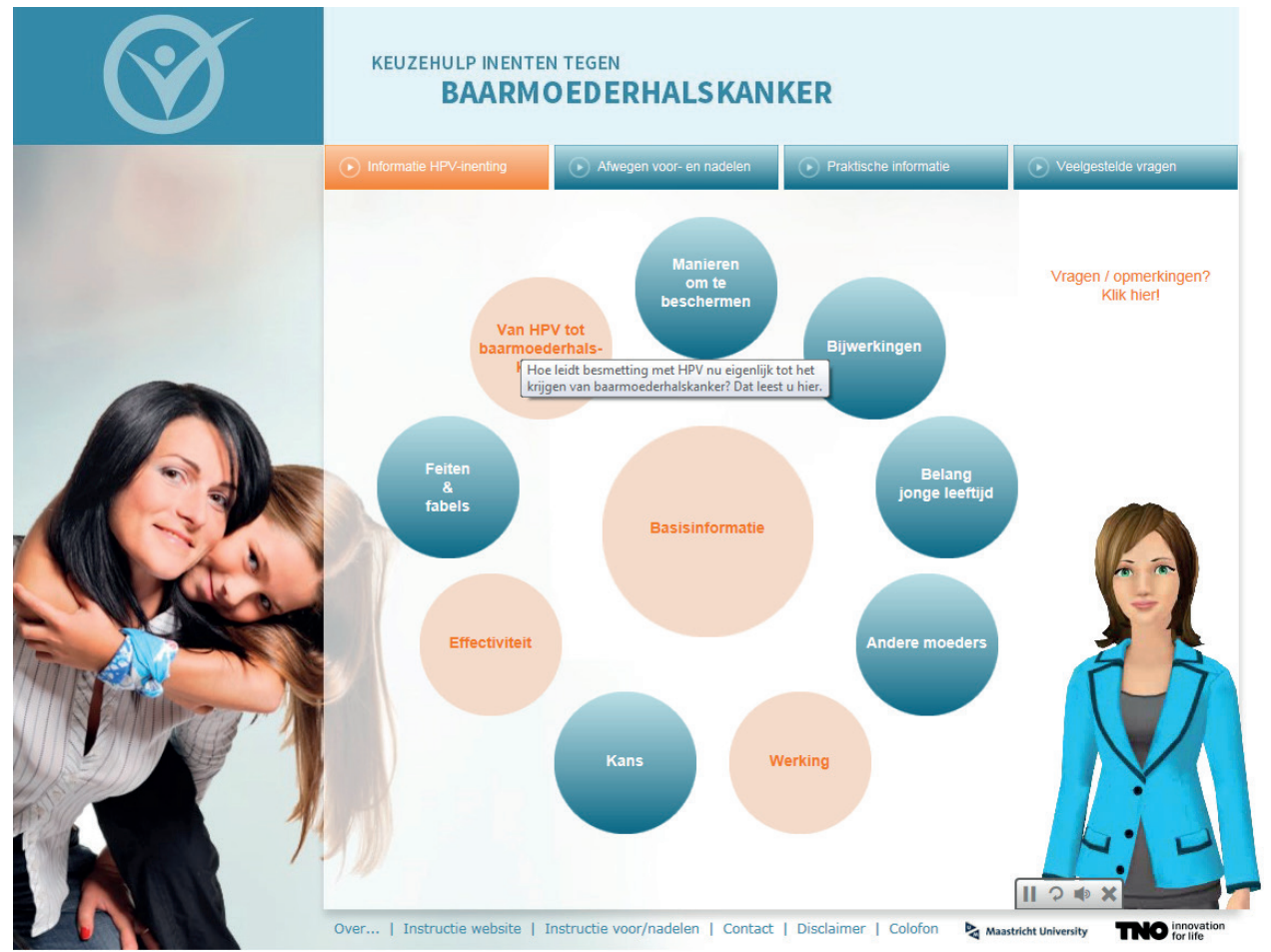

Picture 1: The first menu of the website ('information about the HPV vaccination'). 


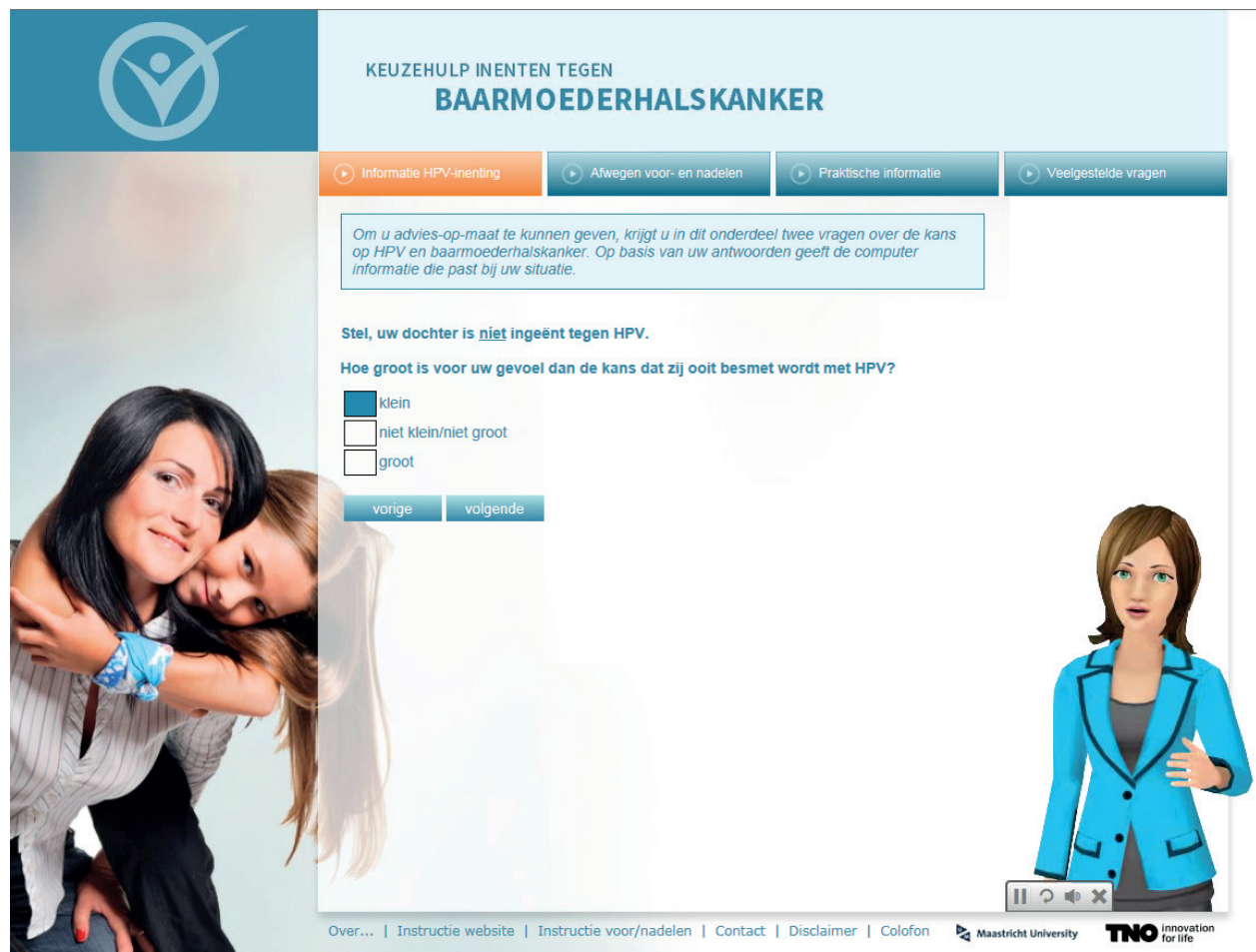

Picture 2: Within the 'risk component' of menu 1, the assistant asks a question about a mothers' risk perception of her daughter getting infected with HPV on which feedback was then tailored (Picture 3). 


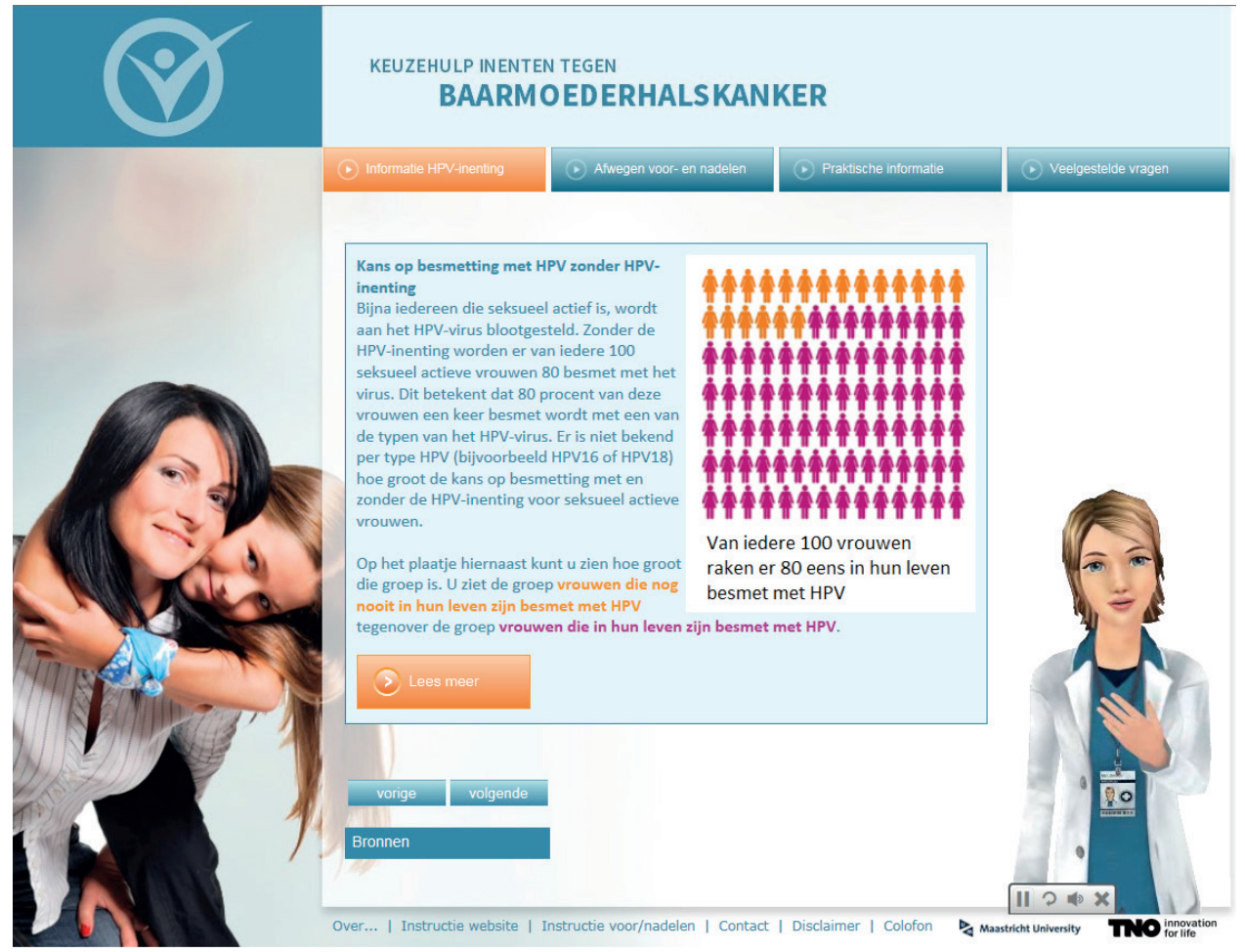

Picture 3: Tailored feedback on mothers' risk perception by the doctor-like assistant. 


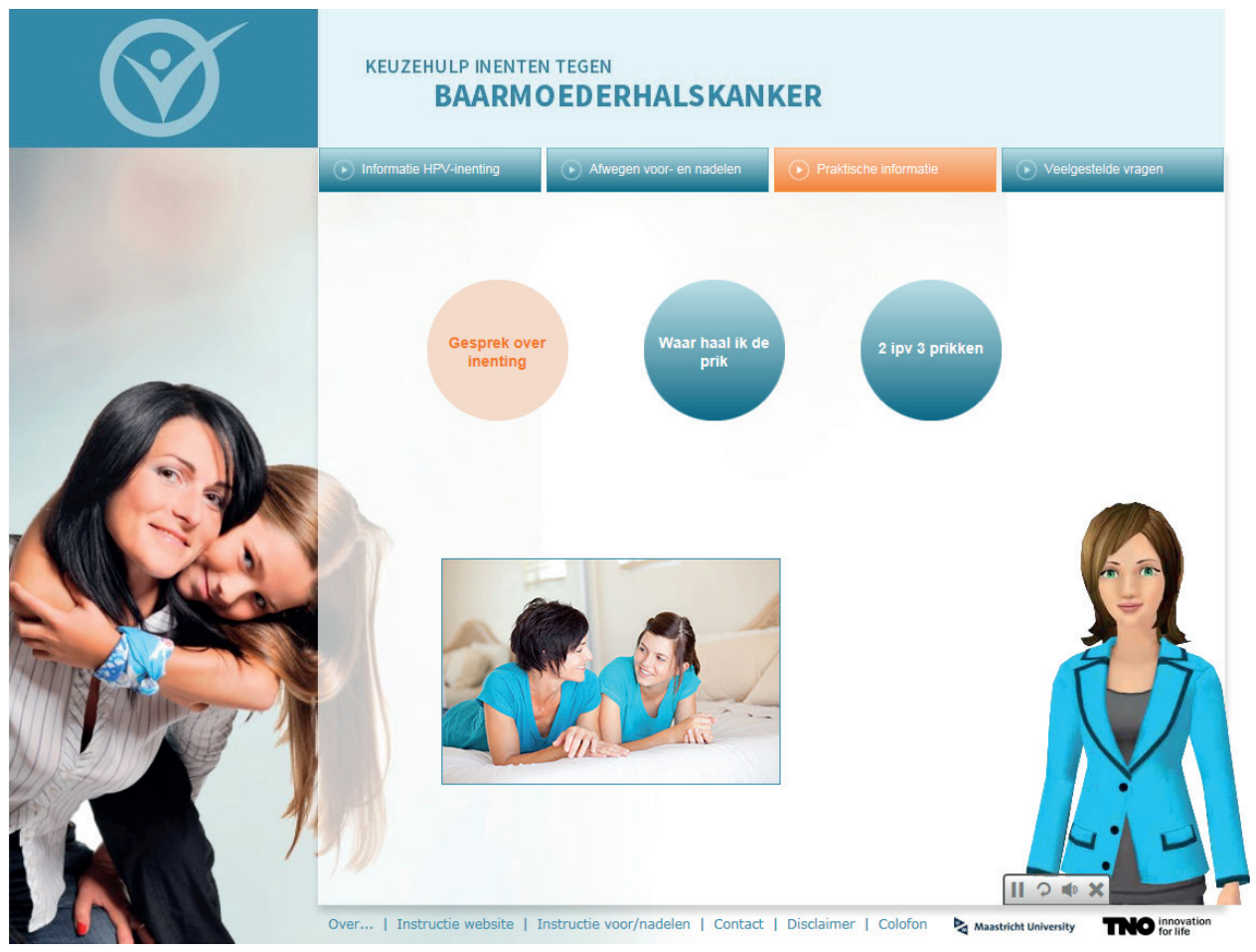

Picture 4: The third menu of the website ('practical information'). 


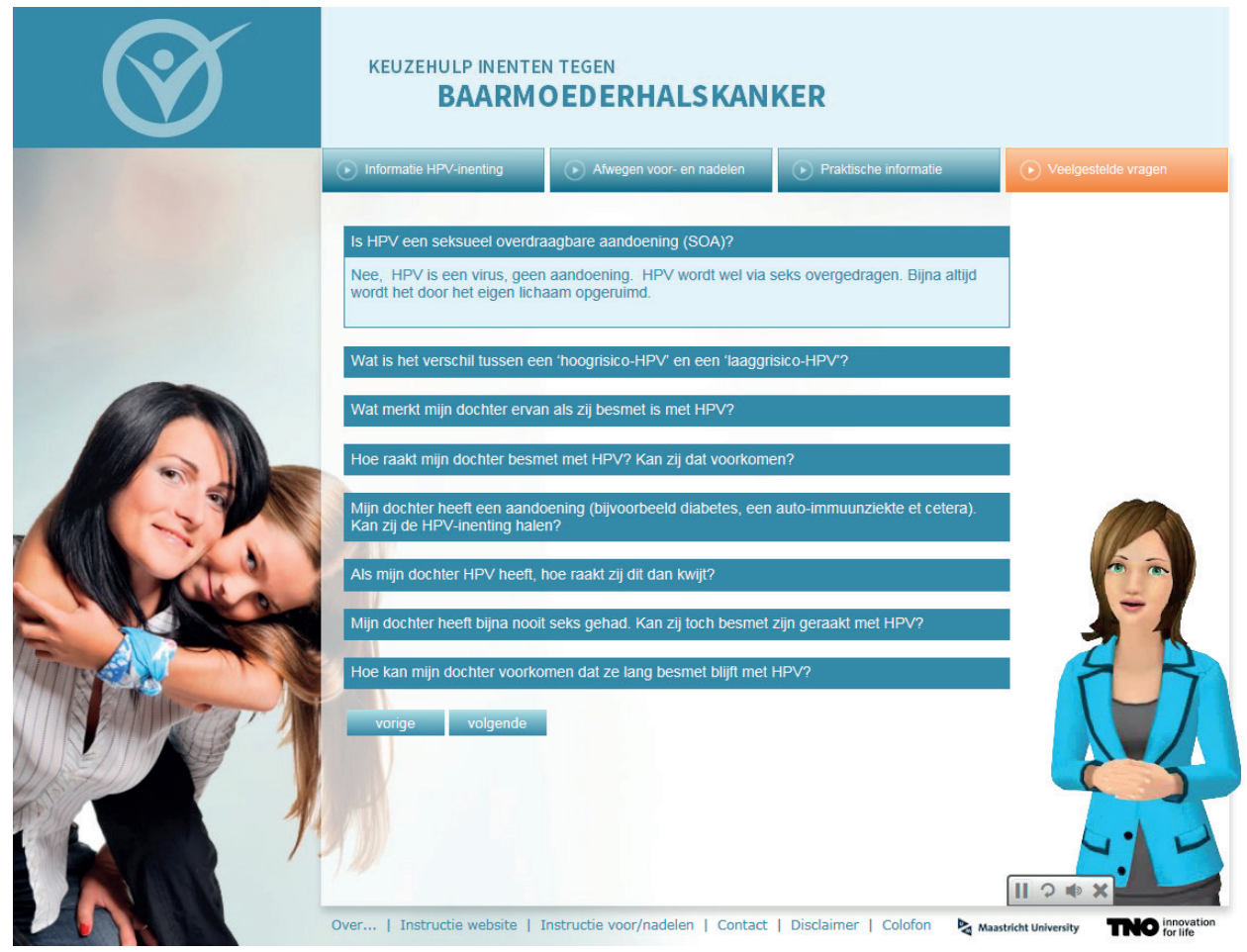

Picture 5: The fourth menu of the website ('frequently asked questions'). 


\section{VALORIZATION ADDENDUM}

This section describes the social and economic relevance of this research project in addition to the scientific relevance. This will be done by pointing out the relevance of the project and the Web-based tailored intervention (i.e., the final product), the target groups, the innovative aspect of the intervention, and how the results are currently being implemented.

As is explained in this $\mathrm{PhD}$ thesis, since 2010, Dutch 12-year-old girls are invited to receive the vaccination against Human Papilloma Virus (HPV) on a yearly basis. Infection with HPV can cause cervical cancer. However, HPV vaccination uptake remains to be low (i.e., $46 \%$ in 2017). The uptake needs to be improved in order to reduce the cervical cancer burden. This thesis generates insight into the systematical development and evaluation of a Web-based, tailored educational intervention (with virtual assistants) promoting HPV vaccination acceptability among mothers of invited girls. The intervention has the potential to improve HPV vaccination acceptability and informed decision-making, to decrease decisional conflict, as well as to motivate HPV vaccination uptake among mothers of invited girls. In addition, the Web-based, tailored intervention has potential for broad scale dissemination and implementation at relatively low costs. Therefore, it can have substantial impact at the Dutch population level.

The results of the studies are of interest for several target groups. First, for mothers of invited girls because the intervention can help them (and their daughters) making an informed decision about HPV vaccination. Second, for invited girls themselves and their (other) relatives, because vaccinated girls have a decreased risk of getting infected with HPV and developing cervical cancer later in life. Third, for stakeholders in policy (i.e., the ministry of Health, Welfare and Sports, RIVM, the Dutch Health Council, and Netherlands Centre of Youth and Health), because vaccination pursues their goal of decreasing morbidity, mortality, and socioeconomic costs associated with cervical cancer cases. Besides, RIVM is now able to improve their effort in promoting HPV vaccination acceptability by implementing this evidence-based educational intervention. Finally, health care professionals may feel supported and strengthened in the delivery of the HPV vaccination.

The intervention can be called highly innovative, because of the selected behavioral change techniques, including the use of virtual assistants, which were combined within a Webbased tailored program promoting HPV vaccination acceptance. And so far, this is the first intervention promoting HPV vaccination acceptability that has been successfully developed in a systematic manner by using the Intervention Mapping protocol. 
As the intervention appeared effective, it will be incorporated into the national HPV vaccination communication from 2019 onwards. There have been several meetings between TNO, Maastricht University, and RIVM to prepare the implementation of the Web-based tailored education. The intervention has recently been transferred from TNO to RIVM; they now have full control and management over the website. The website is already accessible at a secured platform, but will soon become free available for the general public. Hosting costs are low, especially compared to the large groups of people that can be reached by the intervention. When it has become publicly available, time and money remain necessary for keeping content up-to-date and for monitoring use and effectiveness of the intervention. One of the spin offs could be the development of similar educational interventions targeting other childhood vaccinations and/or other target groups. Furthermore, we apply for a quality assessment by The Centre for Healthy Living (in Dutch: Centrum Gezond Leven, CGL) with the aim to include the intervention into their intervention database. This will promote knowledge-awareness about the availability of the intervention among professionals in health promotion practice and research and provide them leads for the development of interventions. And, this will contribute to the development of a cumulative knowledge base in promoting HPV vaccination acceptability.

This $\mathrm{PhD}$ thesis will be made available online in order to distribute and share the insights that we have established with this research project. Hardcopies will also be distributed among academics and among stakeholders that could benefit from the Web-based tailored educational intervention (e.g., the municipal health services, general practices, and hospitals). Also, there have already been several presentations at (inter)national conferences and publications to promote dissemination of the results of this research project. 


\section{SUMMARY}

\section{General Introduction}

Worldwide, cervical cancer is a common health concern among women. Persistent infection by the human papillomavirus (HPV) is the causative agent of cervical cancer. In the Netherlands, new cases are still detected each year, of which a considerable amount has fatal consequences. Therefore, the HPV vaccination was included in the national immunization program (NIP), targeting 12 year old girls. However, HPV vaccination uptake remains to be lower than expected (i.e., $46 \%$ in 2017). The aim of this research project was to systematically develop and evaluate an intervention promoting HPV vaccination acceptance. For this, the Intervention Mapping (IM) protocol was used. IM provides a framework for the planning of theory- and evidence-based health promotion interventions. There are six iterative steps in the IM process: (1) needs assessment; (2) specification of change objectives; (3) selection of theory-based intervention methods and practical applications; (4) production of the intervention program; (5) anticipation of program implementation; and (6) planning of a process and effect evaluation. In line with the IM protocol, the present $\mathrm{PhD}$ thesis is divided into three parts: (1) the needs assessment for explaining HPV vaccination acceptability (Chapter 2), (2) intervention development (Chapters 3 and 4), and (3) evaluation (Chapter $5)$.

\section{PART I: The Needs Assessment}

Previous research in the Netherlands has shown that mothers play the most important role in the decision about their daughters' HPV vaccination. The process of their decision making appeared to be based on rather unstable grounds and they appeared to face decisional conflict. Therefore, the overall goals of the intervention were to improve HPV vaccination uptake and the level informed decision-making, and to decrease decisional conflict.

Chapter 2 describes a longitudinal study into the determinants of HPV vaccination acceptability among mothers of invited girls. The purpose was to falsify earlier crosssectional studies on the social-psychological determinants of HPV vaccination uptake. Results showed that HPV vaccination intention was the only stable predictor of HPV vaccination uptake and that this intention were based in attitude, beliefs, subjective norms, habit, and perceived relative effectiveness of the vaccination. So, these longitudinal findings confirmed the outcomes of earlier cross-sectional studies and provided a strong theoretical basis for targeting the intervention to be developed. 


\section{PART II: Intervention Development}

Computer-tailoring was selected as the basic method for change and virtual assistants were used for delivering the intended interactive tailored feedback. These choices suited the mothers' need for more personalized, interactive feedback about the pros and cons of their daughters HPV vaccination uptake. To maximize usability, a user-centered design strategy was applied including focus groups, online pretests, and experimental pretests. Prototypes were experimentally tested and sequentially refined. Besides, feedback from potential users and stakeholders was gathered from the onset of the development process by means of interviews, pilot-tests, and advisory board meetings.

Chapter 3 describes the experimental pretest of the intervention component targeting mothers' perceived daughters' susceptibility towards HPV-related risks. Two types of risk information (i.e., statistical versus narrative risk information) were tested concerning their relative impact on perceived daughters' susceptibility of HPV-related risks. The results showed that mothers who received statistical information felt their daughters were more susceptible towards HPV than mothers who received no statistical information. These findings implicate that statistical risk information is most effective in increasing mothers' perceived susceptibility of their daughters attracting HPV. Hence, statistical risk information was included in the intervention.

Chapter 4 provides a comprehensive and detailed description of the design rationale behind the intervention. This description shows how we proceeded through each step of IM, providing insight into the theory- and research-based foundations of the many decisions that had to be made during the process of intervention development. This resulting intervention blueprint will aid in interpreting the results of our evaluation studies. Moreover, it will ease comparisons of design rationales across interventions, and may provide leads for the development of other eHealth interventions.

\section{PART III: Intervention Evaluation}

Chapter 5 describes the effect and process evaluation. The aim of this study was to determine whether and how the intervention worked, if the intervention was appropriate for affecting the target population, and whether there were any problems with its implementation. A randomized controlled trial (RCT) was conducted in which exposure to the tailored intervention was compared to exposure to the already existing communication about the HPV vaccination. Results from the effect evaluation showed that the intervention had a significant positive effect on informed decision-making, decisional conflict, and nearly all determinants of HPV vaccination uptake. No condition effects were found as far as uptake was concerned. However, secondary analyses showed a dose response effect; the 
extent of use of the intervention positively impacted HPV vaccination uptake, as well as the mothers' level of informed decision-making about their daughters' immunization. Besides, results from the process evaluation showed that mothers highly appreciated the intervention; the website as a whole and the guidance by the virtual assistants. Objective registrations of program use indicated that $63 \%$ of the invited mothers had visited the website. Taken together, the intervention program showed to have high potential for broad scale dissemination and implementation.

\section{General Discussion}

Chapter 6 summarizes the findings of the presented studies, gives an overview of the strengths and limitations of the research project and provides implications for future research and practice. Some of the strengths include the robust empirical and theoretical foundation for intervention development, the user-centered design and the accompanying preliminary (experimental) pre-tests, and the incorporation of both an effect and process evaluation. Some limitations include the fact that IM is a time-consuming process and that the effect sizes of the intervention were relatively small. Implications for future practice include the development of similar intervention-models for (a) target groups other than the mother (e.g., the daughter or the sons in case they will become a next target group for the HPV vaccination), and (b) other vaccinations (e.g., maternal pertussis vaccination). We also recommend other intervention developers to test both an intervention's efficacy and effectiveness, and to incorporate a process evaluation next to the effect evaluation. Another recommendation for future research concerns a possible complementary impact of changing the mode of delivery. Experiences in other countries have shown the surplus value of a more individual-based delivery of the HPV vaccination relative to the mass group-based delivery as currently practiced in the Netherlands.

In conclusion, this $\mathrm{PhD}$ thesis has provided transparency on the systematic development and evaluation of an innovative, interactive, Web-based, tailored intervention promoting HPV vaccination acceptance. The intervention has potential to improve both HPV vaccination acceptability and informed decision making, and to decrease decisional conflict. Therefore, the Web-based tailored intervention will be incorporated into the national HPV vaccination communication from 2019 onwards. 



\section{SAMENVATTING}

\section{Algemene Introductie}

Baarmoederhalskanker is een bekend gezondheidsprobleem onder vrouwen. Aanhoudende infectie met het Humaan Papillomavirus (HPV) is de veroorzaker van baarmoederhalskanker. In Nederland worden nog steeds elk jaar nieuwe gevallen ontdekt, waarvan een aanzienlijke hoeveelheid een fatale afloop heeft. Daarom is de HPV vaccinatie opgenomen in het Rijksvaccinatieprogramma (RVP), gericht op 12-jarige meisjes. Maar, de HPV vaccinatiegraad blijft lager dan verwacht (46\% in 2017). Het doel van dit onderzoeksproject was om op systematische wijze een interventie te ontwikkelen en evalueren om HPV vaccinatie acceptatie te bevorderen. Hiervoor is het Intervention Mapping (IM) protocol gebruikt. Intervention Mapping biedt een raamwerk voor het plannen van op theorie en wetenschappelijk bewijs gebaseerde gezondheidbevorderende interventies. Er zijn zes iteratieve stappen in het IM proces: (1) needs assessment; (2) specificatie van doelen voor verandering in gedrag; (3) selectie van op theorie gebaseerde methoden en praktische applicaties; (4) productie van het interventie programma; (5) anticipatie van programma implementatie; en (6) plannen van een proces en effect evaluatie. In lijn met het IM protocol, kan deze dissertatie ingedeeld worden in drie delen: (1) de needs assessment voor het verklaren van HPV vaccinatie acceptatie (hoofdstuk 2), (2) interventie ontwikkeling (hoofdstuk 3 en 4), en (3) evaluatie (hoofdstuk 5).

\section{DEEL I: De Needs Assessment}

Eerder onderzoek in Nederland heeft aangetoond dat moeders de belangrijkste rol spelen bij het beslissen over hun dochters' HVP vaccinatie. Het proces van een beslissing maken bleek gebaseerd te zijn op enigszins instabiele gronden en zij ervoeren innerlijk conflict. Daarom waren de algehele doelen van de interventie om de HPV vaccinatiegraad en het niveau van geïnformeerde keuze te verhogen, en om innerlijk conflict te verlagen.

Hoofdstuk twee beschrijft een longitudinale studie waarin determinanten van HPV vaccinatie acceptatie onder moeder van uitgenodigde meisjes worden onderzocht. Het doel was om resultaten uit eerdere cross-sectionele studies te bevestigen. De intentie om te vaccineren bleek de enige stabiele voorspeller van vaccinatiegedrag en deze intentie was gebaseerd op attitude, overtuigingen, subjectieve normen, gewoonte en de relatieve effectiviteit van de vaccinatie. Deze longitudinale bevindingen bevestigde de uitkomsten van eerdere cross-sectionele studies en boden een sterke theoretische basis voor het ontwikkelen van de interventie. 


\section{DEEL II: Interventie Ontwikkeling}

Computer-tailoring was gekozen als basis methode voor verandering en virtuele assistenten zijn gebruikt voor het geven van de interactieve getailorde, feedback. Deze keuzes sloten aan bij de behoefte van moeders aan meer persoonlijke, interactieve feedback over de voor- en nadelen van het al dan niet vaccineren van hun dochter. Om bruikbaarheid te optimaliseren is een gebruikersgericht ontwerp gebruikt, bestaande uit focus groepen en online experimenten. Prototypes zijn op experimentele wijze getest en vervolgens verfijnd. Overigens werd feedback van potentiële gebruikers en stakeholders verzameld vanaf het begin van interventie ontwikkeling met behulp van interviews, pilot-tests en klankbord vergaderingen.

Hoofdstuk drie presenteert een experimentele pre-test van een onderdeel van de interventie gericht op waargenomen vatbaarheid jegens moeders waargenomen HPV-gerelateerde risico's van hun dochter. Twee soorten risico informatie (i.e., statistische versus narratieve informatie) zijn getoetst met betrekking tot hun relatieve impact op het beïnvloeden van moeders hun waargenomen vatbaarheid jegens HPV-gerelateerde risico's van hun dochter. Resultaten lieten zien dat moeders die statistische informatie ontvingen, vatbaarder waren voor het feit dat hun dochter besmet kan raken met HPV dan moeders die geen statistische informatie ontvingen. Deze resultaten duiden erop dat statistische risico informatie het meest effectief is in het verhogen van moeders' waargenomen vatbaarheid van hun dochter om besmet te raken met HPV. Daarom is statistische risico informatie gebruikt in de interventie.

Hoofdstuk vier geeft een uitgebreide, gedetailleerde omschrijving van de ontwikkeling van de interventie. Deze omschrijving laat zien hoe we elke stap van IM hebben doorlopen en geeft daarmee inzicht in de op theorie en onderzoek gebaseerde grondslagen voor de vele beslissingen die gemaakt moesten worden tijdens het ontwikkelen van de interventie. De resulterende interventie blauwdruk zal helpen bij het interpreteren van de evaluatiestudies. Daarnaast zal deze blauwdruk het vergelijken van interventie ontwikkeling tussen interventies vergemakkelijken en kan hij helpen bij de ontwikkeling van andere eHealth interventies.

\section{DEEL III: Interventie Evaluatie}

Hoofdstuk vijf beschrijft de effect en proces evaluatie. Het doel van deze studie was om te bepalen of en hoe de interventie gewerkt heeft, of de interventie de doelgroep succesvol heeft kunnen beïnvloeden en of er problemen waren met de implementatie. Een gerandomiseerd onderzoek met controle groep (RCT) is uitgevoerd waarin blootstelling aan de getailorde interventie werd vergeleken met blootstelling aan de bestaande communicatie over de HPV 
vaccinatie. Resultaten van de effect evaluatie lieten zien dat de interventie een significant positief effect had op geïnformeerde besluitvorming, innerlijk conflict, en bijna alle determinanten van HPV vaccinatie gedrag. Er werden geen conditie effecten gevonden wat vaccinatiegraad betreft. Echter, secondaire analyses lieten een dosis-respons effect zien; de mate waarin de interventie was gebruikt had een positieve invloed op de HPV vaccinatiegraad, en ook op het niveau van geïnformeerde keuze. Overigens lieten resultaten van de proces evaluatie zien dat moeders de interventie zeer positief waardeerden; zowel de website in het algemeen als de begeleiding van de virtuele assistenten. Objectieve registraties van interventiegebruik toonden dat $63 \%$ van de uitgenodigde moeders de website hadden bezocht. Kortom, de interventie lijkt veel potentie te hebben voor verspreiding en implementatie op grote schaal.

\section{Algemene Discussie}

Hoofdstuk zes vat de bevindingen van de gepresenteerde studies samen, geeft een overzicht van de sterktes en zwaktes van het project en geeft implicaties voor toekomstig onderzoek en praktijk. Enkele sterke punten zijn de robuuste, op wetenschappelijk bewijs en onderzoek gebaseerde basis voor interventie ontwikkeling, het gebruikersgerichte ontwerp en de daarbij-horende voorafgaande (experimentele) pretests, en het includeren van zowel een effect als proces evaluatie. Enkele beperkingen zijn het feit dat IM een tijdrovend proces is en dat de effect groottes van de interventie relatief smal waren. Aanbevelingen voor de praktijk zijn de ontwikkeling van soortgelijke interventie-modellen voor (a) doelgroepen anders dan de moeder (zoals de dochter of de zoons indien zij de volgende doelgroep worden voor de HPV vaccinatie) en (b) andere vaccinaties (zoals maternale kinkhoest). Ook bevelen we interventie ontwikkelaars aan om zowel de effectiviteit als de werkzaamheid van de interventie te toetsen, en om een proces evaluatie te includeren naast de effect evaluatie. Een andere aanbeveling voor toekomstig onderzoek is een mogelijk aanvullende invloed door het veranderen van de wijze van levering. Ervaring uit andere landen heeft de toegevoegde waarde van een meer individuele leveringswijze van de HPV vaccinatie getoond, vergeleken met de massa, groeps-gebaseerde leveringswijze zoals die nu in Nederland is.

Concluderend toonde dit proefschrift op transparante wijze de systematische ontwikkeling en evaluatie van een innovatieve, interactieve, online, getailorde interventie ter bevordering van HPV vaccinatie acceptatie. De interventie heeft potentie om zowel HPV vaccinatie acceptatie als geïnformeerde keuze te bevorderen en om innerlijk conflict te verminderen. Daarom zal de online getailorde interventie geïncludeerd worden in de nationale communicatie over de HPV vaccinatie vanaf 2019. 



\section{DANKWOORD}

JAAAA, hij is af! Dit proefschrift is natuurlijk niet alleen door mij tot stand gekomen. Daarom zou ik hier graag alle mensen bedanken die mij hebben geholpen gedurende mijn $\mathrm{PhD}$. De lijst hieronder is ongetwijfeld niet geheel compleet, dus ook aan degene die er niet in vermeld staan: ontzettend bedankt!!

Allereerst natuurlijk mijn (co-)promotoren Rob, Hilde en Theo. Wat heb ik ontzettend veel van jullie geleerd! Rob, je gaf mij altijd een zeer welkom gevoel als ik naar Maastricht kwam. Altijd werd er tijd vrij gemaakt en kwam ik terug met nieuwe, mooi ideeën. Naast de interesse in het onderzoek, ben je ook altijd zeer geïnteresseerd geweest in hoe het met mij ging. Hilde en Theo, ik kon mij geen betere begeleiders bedenken. Jullie stonden altijd klaar voor (extra -indien nodig) overleg en hebben het beste in mij naar boven gehaald. Daarnaast trapten jullie op de rem als ik iets téveel opging in het werk en waren jullie erg begripvol toen het even wat minder ging met mij persoonlijk. Hilde, aan jouw oog voor detail ontging niks en de feedback die ik kreeg maakte mij altijd enthousiast om weer aan de slag te gaan! Theo, jij hebt me echt met een kritische blik naar zowel mijn eigen denken als schrijven laten kijken, wat mijn stukken absoluut tot een hoger niveau heeft getild.

Ik wil ook graag de leescommissie bedanken voor de tijd en aandacht die ze besteed hebben aan het beoordelen van mijn proefschrift. En natuurlijk de duizenden participanten die hebben deelgenomen tijdens het onderzoek.

De rest van het HPV-team vanuit TNO: Jantine, Wilma, Annerieke, Tina en Mark; bedankt voor jullie input tijdens brainstormsessies en het meedenken en -schrijven aan artikelen.

Mijn paranimfen tevens beste vriendinnen, Nina en Pauline. We kennen elkaar al sinds de basisschool en ik ben jullie mega dankbaar voor jullie vertrouwen in mij, de steun die jullie mij altijd gaven -ook wanneer ik er een tikkeltje doorheen zat. Maar natuurlijk ook alle leuke, gezellige momenten tijdens eetdates, park bezoekjes, festivals -noem maar op; allen memorabele momenten.

Hedwig en Renate; jullie waren zowel mijn collega's als vriendinnen als trein-maten. Heerlijk in de vroege uurtjes op naar Leiden of terug naar Amsterdam. Maar daarnaast ook dank voor de amusante momenten tijens o.a. eetdates, op stap, de film en de bingo.

Nog meer (ex) TNO’ers: Anna (een betere roommie kon ik me niet wensen), Eline, Yvonne, Dirk, Janneke, Jorinde, Noortje, Gaby, Joris en Mariëtte. Bij jullie kon ik altijd even mijn hart luchten in stressvolle tijden en daarnaast immer gezelligheid op de werkvloer en natuurlijk aan de lunchtafel of op de $4^{e}$. 
Mijn collega's van UM; ondanks dat ik niet vaak in het zuiden was, vond ik het altijd leuk om er te zijn en jullie te zien! Ook de uitjes waren gezelligheid alom.

Van het RIVM: Marina, Liesbeth, Hester. Jullie waren altijd zeer enthousiast over dit project en dat gaf me veel positieve energie! Ik vind het fantastisch dat de interventie volgend jaar wordt geïmplementeerd. Daarnaast ook Jim en Hans, bedankt voor het meedenken en jullie bijdrage aan de artikelen. Petra, bedankt voor het helpen bij het verzamelen van de data.

Mark en Michael, bedankt voor al jullie hulp met het programmeren van de website. Was er een probleem (bijvoorbeeld een virtuele assistent die op de kop in beeld stond haha)? Altijd binnen no time een reactie en voilá, probleem opgelost. Zonder jullie was de website nooit zo geworden zoals hij nu is!

Eva en Maxine, jullie hebben echt indruk op mij gemaakt met jullie inzet tijdens jullie Master. Eva, ik herinner mij dat wij zelfs om 2 uur's nachts nog aan het skypen waren vlak voor de website online ging om alles te laten werken zoals het betaamde. Maxine, de vele (VELE) analyses die je hebt gedaan en alles waarbij je me hebt geholpen; merci beaucoup!

Charlotte, de laatste twee jaar van mijn $\mathrm{PhD}$ was je mijn steun en toeverlaat. Je hielp me altijd dingen te relativeren en vooral om te beseffen dat werk niet het enige is in het leven.

En tot slot mijn fenomenale familie en vrienden. Frank, ik heb altijd tegen je opgekeken als zijnde hoogleraar en kreeg altijd goede tips. Een speciaal woord van dank aan mam en pap; wat hebben jullie me bijgestaan tijdens het gehele proces. En natuurlijk Thomas Marcus Maria. Jullie hebben mij altijd voor de volle $100 \%$ gesteund en zonder jullie had ik hier never nooit gestaan. Ik ben jullie ontzettend dankbaar! 


\section{CURRICULUM VITAE}

Mirjam Pot was born in Amersfoort, the Netherlands on the 9th of April 1990. After completing her secondary school education at the Johan van Oldebarneveldt Gymnasium in 2008, she started studying Psychology at Universiteit Utrecht. In 2011, she finished her Bachelor in Psychology (Cum Laude) and started the Master Social Psychology at Utrecht University. During the Master, she visited the University of New South Whales for an internship and completion of her thesis at the National Centre in HIV Social Research. In July 2012, she obtained her Master's degree. From 2012 to 2017, she was employed as a PhD Candidate at the Department of Work and Social Psychology of Maastricht University, seconded at the Department of Child Health of the Netherlands Organization for Applied Scientific Research TNO. The results of her PhD-project are described in the present $\mathrm{PhD}$ thesis. She completed her PhD-project under supervision of Prof. Dr. Robert Ruiter (UM), Dr. Hilde van Keulen (TNO), and Dr. Theo Paulussen (TNO). 


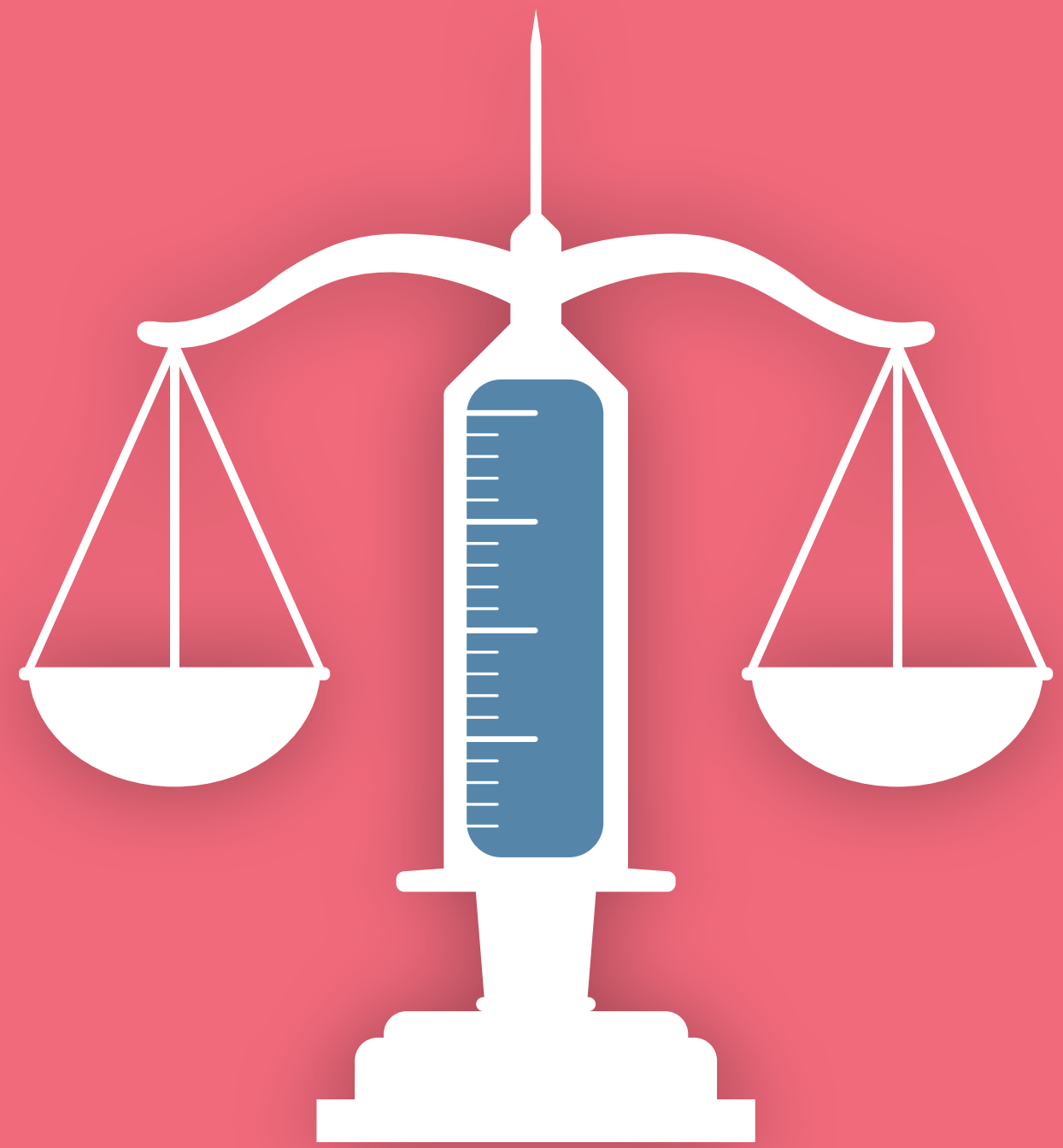

